\title{
Modelling geodetic observations of postseismic displacement in the central and southern South Island of New Zealand
}

By

Pegah Faegh-Lashgary

\author{
A thesis \\ submitted to Victoria University of Wellington \\ in fulfilment of the requirements for the degree of \\ Doctor of Philosophy in Geophysics.
}

Victoria University of Wellington

2016 

"If I were again beginning my studies, I would follow the advice of Plato and start with mathematics."

Galileo Galilei 



\section{Abstract}

The last seven years have seen southern New Zealand affected by several large and damaging earthquakes: the moment magnitude $\left(\mathrm{M}_{\mathrm{W}}\right) 7.8$ Dusky Sound earthquake on 15 July 2009, the $\mathrm{M}_{\mathrm{W}} 7.1$ Darfield (Canterbury) earthquake on 4 September 2010, and most notably the $\mathrm{M}_{\mathrm{W}} 6.2$ Christchurch earthquake on 22 February 2011 and the protracted aftershock sequence. In this thesis, we address the postseismic displacement produced by these earthquakes using methods of satellite-based geodetic measurement, known as Interferometric Synthetic Aperture Radar (InSAR) and Global Positioning System (GPS), and computational modelling.

We observe several ground displacement features in the Canterbury and Fiordland regions during three periods: 1) Following the Dusky Sound earthquake; 2) Following the Darfield earthquake and prior to the Christchurch earthquake; and 3) Following the Christchurch earthquake until February 2015.

The ground displacement associated with postseismic motion following the Dusky Sound earthquake has been measured by continuous and campaign GPS data acquired in August 2009, in conjunction with Differential Interferometric Synthetic Aperture Radar (DInSAR) observations. We use an afterslip model, estimated by temporal inversion of geodetic data, with combined viscoelastic rebound model to account for the observed spatio-temporal patterns of displacement. The two postseismic processes together induce a significant displacement corresponding to principal extensional and contractual strain rates of the order of $10^{-7}$ and $10^{-8} \mathrm{yr}^{-1}$ respectively, across most of the southern South Island.

We also analyse observed postseismic displacement following the Dusky Sound earthquake using a new inversion approach in order to describe afterslip in an elasticviscoelastic medium. We develop a mathematical framework, namely the "Iterative Decoupling of Afterslip and Viscoelastic rebound (IDAV)" method, with which to invert temporally dense and spatially sparse geodetic observations. We examine the IDAV method using both numerical and analytical simulations of Green's functions.

For the post-Darfield time interval, postseismic signals are measured within approximately one month of the mainshock. The dataset used for the post-Darfield displacement spans the region surrounding previously unrecognised faults that ruptured during the mainshock. Poroelastic rebound in a multi-layered half-space and dilatancy recovery at shallow depths provide a satisfactory fit with the observations.

For the post-Christchurch interval, campaign GPS data acquired in February 2012 to February 2015 in four successive epochs and 66 TerraSAR-X (TSX) SAR acquisitions in descending orbits between March 2011 and May 2014 reveal approximately three years of postseismic displacement. We detect movement away from the satellite of $\sim 3 \mathrm{~mm} / \mathrm{yr}$ in Christchurch and a gradient of displacement of $\sim 4 \mathrm{~mm} / \mathrm{yr}$ across a lineament extending from the westernmost end of the Western Christchurch Fault towards the eastern end of the Greendale East Fault. The postseismic signals following the Christchurch earthquake are mainly accounted for by afterslip models on the subsurface lineament and nearby faults. 



\section{Acknowledgements}

Three years and three offices later I find myself nearing the completion of my thesis. It hasn't been without its ups and downs, speed bumps and roadblocks. My PhD has certainly been an interesting and pivotal point in my life. I am sure IT Support have been waiting for this moment too, what with the hard drive failure, multiple requests for upgrading hardware, oh and crashing the scientific computing cluster (apparently my code was over 9000).

When I was not at the VUW campus, I was at GNS processing the finite element codes. I would have to say the most interesting day at GNS was the great flood of 15th May 2015 - where I was not able to get back into Wellington until very late at night (and not by swimming). There was also that one time where I found myself locked in the building.

Not all my time was spent in Wellington. I have been fortunate enough to attend three conferences in three different countries: AGU in San Francisco, U.S.A, EGU in Vienna, Austria, and Summer School in Yamanakako, Japan. Each place was a unique experience of its own and I would like to attend more events in future. Overall, my time with Victoria University of Wellington, and New Zealand in general have been wonderful. Without further ado, I would like to acknowledge a number of key people.

First and foremost, I wish to thank my primary supervisor AProf. John Townend of Victoria University of Wellington for his support, guidance, patience and above all, his knowledge of all things Geophysics. John is one of the most intelligent people I have met and has had a great influence on me. I am very grateful to have had him as my supervisor.

Furthermore, I would like to thank Dr. Charles Williams for his guidance, excellence in programming, and debugging skills. Charles has been a great supervisor and has always been there when I have needed assistance using his code libraries.

I thank Dr. Ian Hamling for reviewing my papers in a timely manner. Ian has without a doubt made my papers read well.

I thank Dr. Mahdi Motagh from GFZ, Potsdam for arranging the opportunity for my $\mathrm{PhD}$ in New Zealand. Mahdi has always been prompt to reply to my emails (even at 3am - Berlin time). Mahdi's guidance and managerial finesse helped me keep on track.

At this juncture, I would also like to thank the Earth Quake Commission. Without the funding of EQC, this work would not be possible.

Among other people to thank are my proof readers. My flatmate and good friend Jorge who, while failing School-C English, spent countless hours helping me with corrections. My then-office mate Holly who was an absolute machine with grammar. Dominic, the boy from down the hallway who floats around our office, giving us company and cheering me up during difficult times. My office mate Hubert, who was able to decipher John's handwriting - who knew he had such skills in cryptanalysis?

Last but not least I would like to thank my Mum and Dad over in Iran who I miss very much. They have been very supportive and understanding. Also my husband Nick who has been by my side, keeping me fed, and making me laugh. 



\section{Contents}

$\begin{array}{ll}\text { Abstract } & \text { i }\end{array}$

Contents $\quad$ v

List of Figures $\quad$ ix

List of Tables $\quad$ xi

Abbreviations xiv

1 Introduction 1

1.1 Geological and tectonic setting of the South Island of New Zealand . . 1

1.2 Recent seismicity in the South Island of New Zealand . . . . . . . . . . 3

1.3 Motivations and thesis objectives . . . . . . . . . . . . 7

1.4 Thesis outline . . . . . . . . . . . . . . . . . . . . . 8

2 Measuring and modelling postseismic displacement 13

2.1 Measuring postseismic displacement . . . . . . . . . . . . . . . . . . 13

2.1.1 Interferometric Synthetic Aperture Radar (InSAR) . . . . . . . 13

2.1.2 Global Positioning System (GPS) . . . . . . . . . . . . . . . 21

2.2 Modelling postseismic displacement . . . . . . . . . . . . . . . 24

2.2.1 Viscoelastic relaxation of ductile materials . . . . . . . . . . 25

2.2 .2 Transient creep . . . . . . . . . . . . . . . . . . 39

2.2.3 Viscoelastic cycle models . . . . . . . . . . . . . . . . . 45

2.2.4 Poroelastic rebound of a porous medium . . . . . . . . . . . 48

2.2.5 Shallow fault zone dilatancy recovery . . . . . . . . . . . 56

2.2.6 Numerical computation using the Finite Element Method (FEM) 58

3 Post-Dusky Sound displacement $\quad 63$

3.1 Introduction . . . . . . . . . . . . . . . . . . . . 64

3.1.1 Tectonic setting . . . . . . . . . . . . . . . . 65

3.1.2 Recent seismicity in southern New Zealand . . . . . . . . . . . 68

3.2 Methods . . . . . . . . . . . . . . . . . . . 69

3.2.1 Observations . . . . . . . . . . . . . . . . . . . 69

3.2.2 Modelling time-dependent afterslip . . . . . . . . . . . . . . 71

3.2.3 Modelling coupled viscoelastic rebound and afterslip . . . . . . 77

3.3 Results . . . . . . . . . . . . . . . . . . . . . . . . . . 79

3.4 Discussion . . . . . . . . . . . . . . . . . . . . . . . 90

3.4.1 Alternative viscoelastic models . . . . . . . . . . . . . 90

3.4.2 Coupled viscoelastic rebound and afterslip . . . . . . . . . . . . 92

3.4.3 Stress and strain changes in Canterbury . . . . . . . . . . . . . 92

3.5 Conclusions . . . . . . . . . . . . . . . . . . . . 97

4 Coupling afterslip and viscoelastic rebound $\quad 99$ 
4.1 Introduction . . . . . . . . . . . . . . . . . . . 100

4.2 Methods . . . . . . . . . . . . . . . . . . 102

4.2.1 Mathematical framework . . . . . . . . . . . . . . . 102

4.2.2 Iterative Decoupling of Afterslip and Viscoelastic relaxation (IDAV) 106

4.3 Analytical simulation . . . . . . . . . . . . . . . . . . . . . 109

4.4 Post-Dusky Sound displacement . . . . . . . . . . . . . . . . . . 111

4.5 Numerical simulation . . . . . . . . . . . . . . . . . . . . . . . 115

4.6 Discussion . . . . . . . . . . . . . . . . . . . . . . . . . . 121

4.7 Conclusions . . . . . . . . . . . . . . . . . . . . . 122

5 Post-Canterbury displacement $\quad 123$

5.1 Introduction . . . . . . . . . . . . . . . . . . . . . 124

5.2 Methodology . . . . . . . . . . . . . . . . . 127

5.2 .1 Observations . . . . . . . . . . . . . . . 127

5.2.2 Analytical and numerical models . . . . . . . . . . . . . . 132

5.3 Post-Darfield displacement . . . . . . . . . . . . . . . . . . . 135

5.3 .1 InSAR observations . . . . . . . . . . . . . . . . . 135

5.3 .2 Modelling results . . . . . . . . . . . . . . . 136

5.4 Post-Christchurch displacement . . . . . . . . . . . . . . . . . . 142

5.4.1 GPS and InSAR observations . . . . . . . . . . . . . . . 142

5.4 .2 Modelling results . . . . . . . . . . . . . . . . . 145

5.5 Discussion . . . . . . . . . . . . . . . . . . . . 155

5.6 Conclusions . . . . . . . . . . . . . . . . . . . . . . . . 157

6 Summary and recommendations $\quad 159$

6.1 Post-Dusky Sound displacement . . . . . . . . . . . . . . . . . . . 159

6.2 Coupling afterslip and viscoelastic rebound . . . . . . . . . . . . . 161

6.3 Post-Canterbury displacement . . . . . . . . . . . . . . . . . 162

6.4 Unanswered questions from Section $1.3 \ldots \ldots$. . . . . . . . . 164

$\begin{array}{ll}\text { Appendices } & 167\end{array}$

$\begin{array}{ll}\text { A Coulomb stress changes } & 169\end{array}$

B Global compilation of viscoelastic rebound models $\quad 171$

$\begin{array}{ll}\text { C Post-Dusky Sound supplementary files } & 181\end{array}$

$\begin{array}{ll}\text { D Post-Canterbury supplementary files } & 185\end{array}$

$\begin{array}{ll}\text { Bibliography } & 187\end{array}$ 


\section{List of Figures}

1.1 Basement geology map of New Zealand . . . . . . . . . . . . . . . . . 2

1.2 Recent studies of seismicity in the South Island of New Zealand . . . . 5

1.3 Temporal and spatial scales associated with various fault-related processes 8

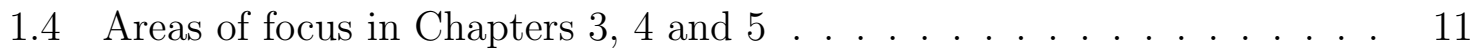

2.1 Schematic illustration of interferometric geometry . . . . . . . . . . . 14

2.2 Measuring surface displacement using radar interferometry . . . . . . . 16

2.3 Interferometry workflow . . . . . . . . . . . . . . . . 17

2.4 Mean line of sight velocity measured from ASAR/Envisat ascending orbits prior to the 4 September 2010 Darfield earthquake . . . . . . . .

2.5 Mean line of sight velocity measured from COSMO-SKyMed ascending orbits following the Darfield earthquake and prior to the Christchurch earthquake . . . . . . . . . . . . . . . . .

2.6 Navigation solution for GPS measurements, multipath and differential

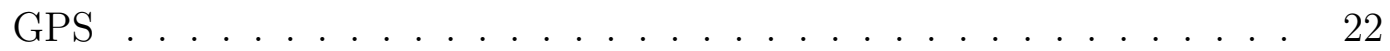

2.7 Schematic illustration of atomic displacement and lattice deformation . 26

2.8 Schematic illustration of different rheology models . . . . . . . . . . . . 29

2.9 Propagator matrix method . . . . . . . . . . . . . . . . . . . . . . . . . . . 32

2.10 Multi-layered viscoelastic model . . . . . . . . . . . . . . . . . . . . . . . . . . . . 34

2.11 Viscoelastic models across the world . . . . . . . . . . . . . . . 40

2.12 Spatial and temporal basis functions used to model slip rates throughout the Akaike's Bayesian Information Criterion (ABIC) method . . . . . . 44

2.13 Earthquake cycle model in an elastic-viscoelastic medium . . . . . . . . 47

2.14 Spring-slider system . . . . . . . . . . . . . . . . . 48

2.15 Fluid flow through porous medium formulted by Darcy's law. . . . . . . 50

2.16 Poroelastic rebound of a simulated rupture on a vertical fault . . . . . 55

2.17 Poroelastic rebound of a simulated rupture within a step-over . . . . . 56

2.18 Shallow fault zone dilatancy recovery . . . . . . . . . . . . . 58

2.19 Heat transfer through two materials with three interfaces . . . . . . . . 60

2.20 Examples of tedrahedral mesh geometries generated in Trelis . . . . . . 61

2.21 Quadratic tetrahedral element as a shape fucntion used in the Finite Element Method (FEM) . . . . . . . . . . . . . . . . 61

3.1 Tectonic setting of the Fiordland region and focal mechanisms of recent earthquakes . . . . . . . . . . . . . . . . 67

3.2 Stack of residuals pre-Dusky Sound earthquake from 22 sites. . . . . . . 71

3.3 Selection of seasonal models . . . . . . . . . . . . . . . . . 72

3.4 cGPS displacement observations of post-Dusky Sound displacement . . 73

3.5 Flowchart illustrating the iterative approach used for coupling timedependent afterslip and viscoelastic rebound . . . . . . . . . . . . . 79

3.6 Viscoelastic displacement time series induced by the Dusky Sound earthquake for a Maxwell rheology and a Burger's body . . . . . . . . . . . . 80 
3.7 Accumulated displacement due to the viscoelastic rebound of a Burger's

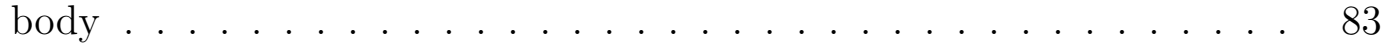

3.8 Temporal modes of post-Dusky Sound displacement . . . . . . . . . . . 84

3.9 Slip models of post-Dusky sound displacement . . . . . . . . . . . . . 85

3.10 Fits with the post-Dusky Sound displacement acquired by campaign measurements . . . . . . . . . . . . . . . . 85

3.11 Fits with the post-Dusky Sound displacement measured by ALOS SAR acquisitions . . . . . . . . . . . . . . .

3.12 Fits of the coupled afterslip and viscoelastic rebound model with the post-Dusky Sound cGPS displacement time series . . . . . . . . . . . .

3.13 Accumulated displacement due to coupled afterslip with viscoelastic rebound of a Maxwell rheology . . . . . . . . . . . . . . . . . . .

3.14 Post-Dusky Sound Coulomb stress models associated with the Greendale and Charing Cross faults . . . . . . . . . . . .

3.15 Post-Dusky Sound strain ellipses observed from 16 cGPS sites in the South Island of New Zealand . . . . . . . . . . . . . . . . . .

3.16 Post-Dusky Sound principal strain components estimated by coupled time-dependent model (Option 4$) \ldots \ldots \ldots$

4.1 Methodology used to obtain unique solutions for the elastic-viscoelastic Sylvester Equation 4.29 . . . . . . . . . . . . . . . . . . . . 108

4.2 Analytically simulated elastic and viscoelastic displacements . . . . . . 110

4.3 Constrained and unconstrained decompositions and solutions of the Sylvester

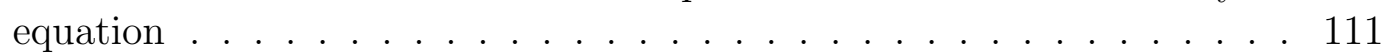

4.4 Simulated and estimated slip models of analytical case study . . . . . . 112

4.5 Fits to the analytically simulated displacement . . . . . . . . . . . . . . 112

4.6 Fiordland and Canterbury regions and focal mechanisms of recent earthquakes . . . . . . . . . . . . . . . . . . 113

4.7 Arbitrary viscoelastic Green's functions of different viscosities . . . . . 114

4.8 Convergence of elastic and viscoelastic time functions and slip models of post-Dusky Sound displacement with different viscosities . . . . . . . 116

4.9 Slab geometry . . . . . . . . . . . . . . . . . . . . . 117

4.10 Numerically calculated Green's functions as responses to unit-step slip on a simulated fault . . . . . . . . . . . . . . . . . 118

4.11 Numerically simulated elastic and viscoelastic displacements . . . . . . 119

4.12 Convergence of elastic and viscoelastic time functions of numerically simulated displacement . . . . . . . . . . . . . . 120

4.13 Slip models of numerically simulated displacement . . . . . . . . . . . . 120

5.1 Tectonic setting of the South Island of New Zealand and focal mechanisms of recent earthquakes in the Canterbury region . . . . . . . . . . 128

5.2 GPS horizontal velocities following the Christchurch earthquake . . . . 130

5.3 Post-Darfield unwrapped interferograms acquired from ascending and descending COSMO-SkyMed orbits . . . . . . . . . . . . . . 131

5.4 Observed time series of post-Darfield displacement . . . . . . . . . . . 132

5.5 Post-Darfield unwrapped interferogram acquired from descending TerraSARX orbits . . . . . . . . . . . . . . . . . . . 133

5.6 Mean Line Of Sight velocities following the Christchurch earthquake . . 134

5.7 Profiles of displacement across the Greendale East fault . . . . . . . . . 137

5.8 Post-Darfield rate-strengthening afterslip . . . . . . . . . . . . . . . . . 139 
5.9 Post-Darfield afterslip model by Motagh et al. (2014a) . . . . . . . . . 140

5.10 Post-Darfield poroelastic rebound models for different parameterisations 142

5.11 Comparision of poroelastic rebound and observed time series of displacement within the Greendale step-over . . . . . . . . . . . . . . . . 143

5.12 Post-Darfield Mogi point sources . . . . . . . . . . . . . . . . . . 143

5.13 Observed, modelled and residual post-Darfield displacements . . . . . . 144

5.14 Post-Christchurch InSAR and GPS observations . . . . . . . . . . . . 145

5.15 Post-Christchurch rate-strengthening afterslip on the subsurface lineament 148

5.16 Post-Christchurch rate-strengthening afterslip on the subsurface lineament and the nearby faults . . . . . . . . . . . . . . . . . 149

5.17 Post-Christchurch slip models . . . . . . . . . . . . . . . . 150

5.18 Fits to the GPS observations of post-Christchucrh displacement . . . . 151

5.21 Post-Christchurch poroelastic rebound . . . . . . . . . . . . . . . 154

5.22 Post-Christchurch Mogi point sources . . . . . . . . . . . . . . . 155

5.23 Observed, modelled and residual post-Christchurch displacements . . . 156

A.1 Fault-plane coordinate system . . . . . . . . . . . . . . . . . . . 169

C.1 Fits to the post-Dusky Sound cGPS displacement time series with a viscosity of $2 \times 10^{18} \mathrm{~Pa} \cdot \mathrm{s} \ldots \ldots \ldots$. . . . . . . . . . . . 184

D.1 Coseismic displacement due to the 13 June 2011 earthquake in Christchurch 185

D.2 Coseismic displacement due to the 23 December 2011 earthquake in Christchurch . . . . . . . . . . . . . . . . 186

D.3 Standard deviation of the post-Christchurch mean line of sight velocity acquired from TerraSAR-X images . . . . . . . . . . . . 186 



\section{List of Tables}

2.1 Mechanical analogue model and material behaviour for viscoelastic materials .......................... 30

2.2 Maxwell and Standard Linear Solid materials _ . . . . . . . . . . . . 31

3.1 Root Mean Square Errors of viscoelastic models: Post-Dusky Sound displacement . . . . . . . . . . . . . . . . . . 91

4.1 Table of nomenclature used to describe coupling method . . . . . . . . 102

4.2 Root Mean Square Errors of varied coupled models: Post-Dusky Sound displacement . . . . . . . . . . . . . . . . . . 115

5.1 Post-Canterbury interferometric characteristics . . . . . . . . . . . . . 129

5.2 Model parameters of poroelastic rebound mechanism . . . . . . . . . . 141

5.3 Root Mean Square Errors of post-Christchurch slip models . . . . . . . 154

A.1 Table of nomenclature used to describe Coulomb stress models . . . . . 170

B.1 Global rheology database . . . . . . . . . . . . . . . . . . . . 174

B.2 Continuation of global rheology database: Viscoelastic models and viscosity values . . . . . . . . . . . . . . . . . . 177

B.3 Continuation of global rheology database: Range of layers in depths . . 180 



\section{Abbreviations}

ABIC Akaike's Bayesian Information Criterion ........................... 43

ALOS Advanced Land Observation Satellite .......................... 4

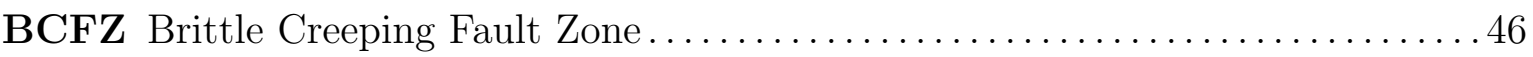

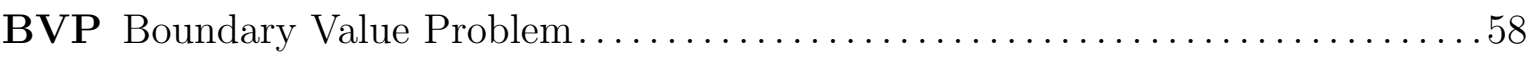

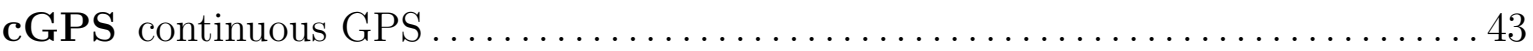

CSK COSMO-SkyMed.......................................... 162

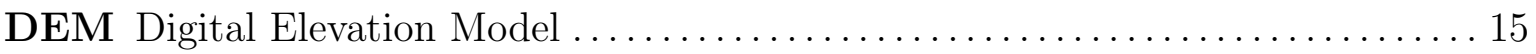

FEM Finite Element Method ................................... 161

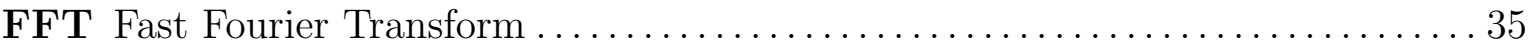

GPS Global Positioning System ................................... 13

ICAIM Independent Component Analysis Inversion Method .................. 161

IDAV Iterative Decoupling of Afterslip and Viscoelastic relaxation ............. 161

InSAR Interferometric Synthetic Aperture Radar ........................... 13

LiDAR Light Detecting And Ranging............................... 124

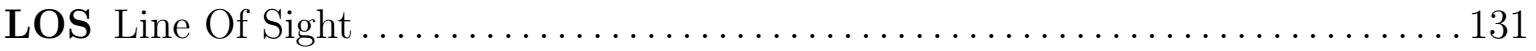

MCMKF Monte Carlo Mixture Kalman Filter ............................ 43

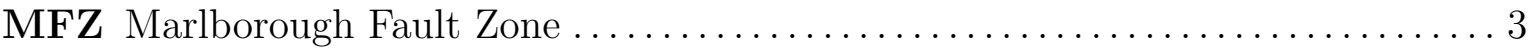

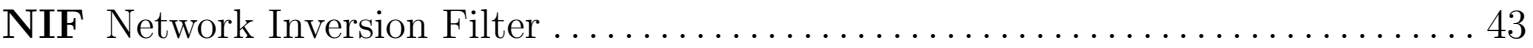

PCAIM Principal Component Analysis-based Inversion Method ............... 76

PDEs Partial Differential Equations................................... 58

PS Permanent Scatterer ............................................. 19

PS-InSAR Permanent Scatterer-InSAR ................................ 16

RMSE Root Mean Square Error ....................................... 140

SBAS Small BAseline Subset .................................... 129 


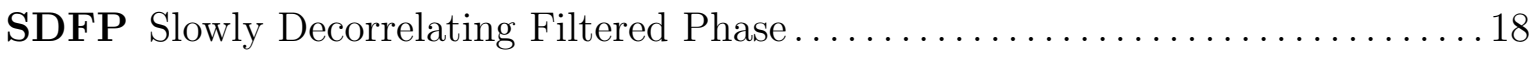

SISZ South Iceland Seismic Zone................................... 53

SLC Single Look Complex .......................................... 15

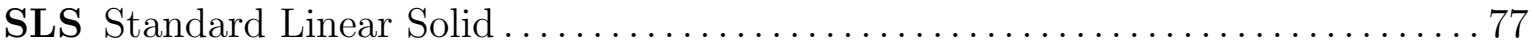

SRTM Shuttle Radar Topography Mission ............................. 15

StaMPS Stanford Method for Permanent Scatterers ....................... 18

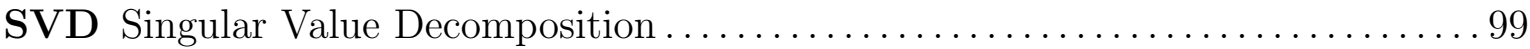

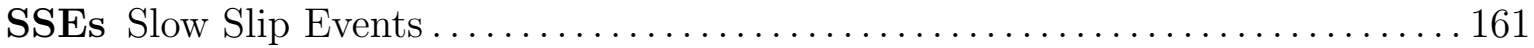

SAR Synthetic Aperture Radar . . . . . . . . . . . . . . . . . . . . . . . . . . . . 14

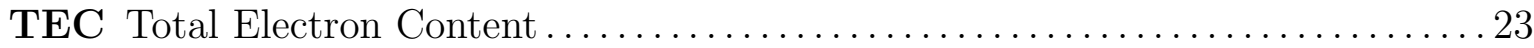

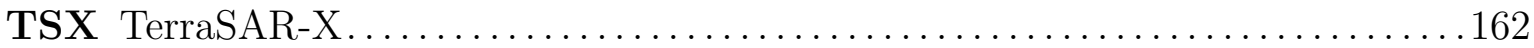




\section{Chapter 1}

\section{Introduction}

This thesis contributes to the methods of measuring, modelling and interpreting postseismic ground displacement following the 15 July 2009 Dusky Sound earthquake and the Canterbury earthquake sequence of 2010-2012 in the central and southern South Island of New Zealand. We study a 400-day time interval following the Dusky Sound earthquake and spanning more than three years of the postseismic period after the 22 February 2011 Christchurch earthquake. The interval studied has seen tens of thousands of reported $\mathrm{M}_{\mathrm{W}}>2$ earthquakes ${ }^{1}$ with the most significant events being the Dusky Sound earthquake and the Canterbury earthquake sequence of 2010-2012. In this chapter, we describe the tectonic and geological setting and the recent seismicity in the South Island of New Zealand as an introduction to this research. We provide a brief introduction to study areas and the underlying motivations, objectives and outline of this thesis.

\subsection{Geological and tectonic setting of the South Is- land of New Zealand}

New Zealand was contiguous with Australia and Antarctica before the Late Cretaceous (Mortimer, 2004). Accretion onto the Gondwana plate margin during the Paleozoic and Mesozoic formed the New Zealand fragment (Mortimer, 2004; Cox and Sutherland, 2007). Extension during the Cretaceous to Paleogene separated the fragment from Australia and Antarctica as a thinned sub-continental crust (Mortimer, 2004; Cox and Sutherland, 2007).

The basement of the South Island of New Zealand is presently divided into Eastern and Western Province Terranes (Bishop, 1985; Bradshaw, 1989). The dominant feature of the Western Province, extending from Fiordland to west Nelson, is the metamor-

\footnotetext{
${ }^{1}$ http://quakesearch.geonet.org.nz/
} 

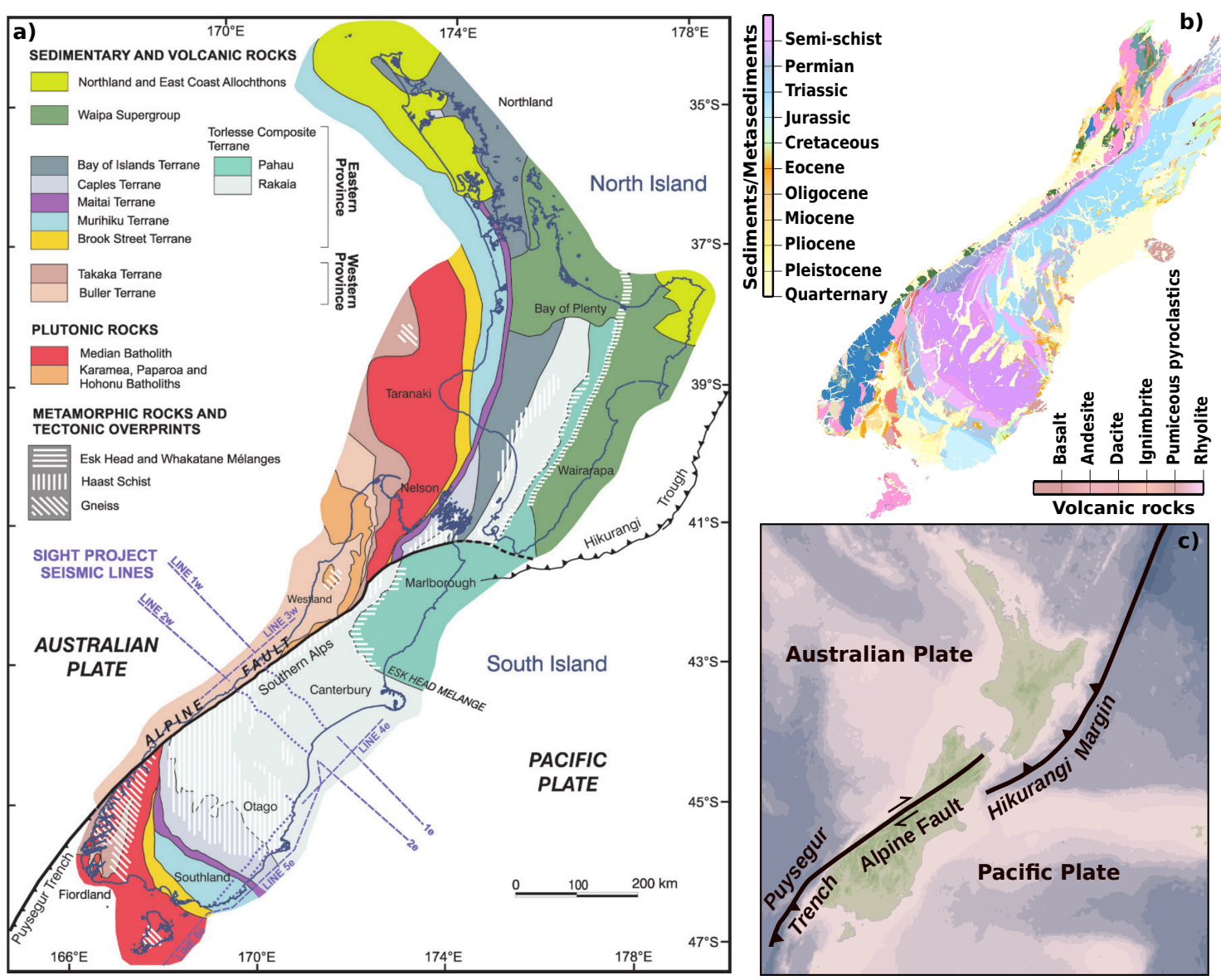

Figure 1.1: a) Basement geology map of New Zealand (Cox and Sutherland, 2007) adapted from (Mortimer, 2004) b) Regional geological map, data source: GNS Science c) AustralianPacific plate boundary.

phosed Buller Terrane (Nathan, 1976), which amalgamated with Takaka Terrane in the earliest Devonian (Figure 1.1a; Cooper and Tulloch, 1992). The Buller Terrane consists mainly of Late Cambrian to Late Ordovician quartzose sediments (Nathan, 1976). Metamorphosed Paleozoic sedimentary and volcanic rocks constitute the Takaka Terrane (Cooper, 1989). The Eastern Province Terranes, extending from Otago to East Cape, containing Permian to Jurassic sedimentary rocks, are dominated by the Rakaia and Pahau Terranes (Figure 1.1a; Mortimer, 2004). The Rakaia Terrane was overprinted by metamorphism during the Jurassic to Early Cretaceous forming the Haast Schist. The Pahau Terrane contains younger Late Jurassic to Early Cretaceous Torlesse quartzofeldspathic greywacke that underwent later metamorphism (Figure 1.1a and b; MacKinnon, 1983; Wandres et al., 2004, 2005).

The Australian-Pacific plate boundary, with current high rates of motion, was formed during the Palaeogene and Neogene (Cox and Sutherland, 2007). We summarise the main features of the present New Zealand plate tectonic from northernmost to southernmost as follows (Figure 1.1c): 
- Subduction of the Pacific plate along the Hikurangi Margin beneath the North Island (Barnes and Lépinay, 1997; Barnes et al., 1998; Reyners, 1998; EberhartPhillips and Reyners, 1999; Wallace et al., 2004; Barker et al., 2009);

- Transition to strike-slip along the Alpine Fault (Van Dissen and Yeats, 1991; Holt and Haines, 1995; Beavan et al., 1999; Norris and Cooper, 2001); and

- Eastward subduction of the Australian plate beneath the Pacific plate along the Puysegur Trench (Norris et al., 1990).

The principal fault structure in the South Island is the Alpine Fault, which accommodates $\sim 70 \%$ of relative motion between the Australian and Pacific plates (Berryman et al., 1992; Sutherland and Norris, 1995; Beavan et al., 1999) and along with the Marlborough Fault System connects the northern and southern subduction zones (Berryman et al., 1992). Sutherland et al. (2000) proposed that the continental collision zone is a result of strike-slip motion since $\sim 25 \mathrm{Ma}$ that has localised shear strain near the Alpine Fault. The link between the northern segment of the Alpine Fault and the Hikurangi Margin is in the Marlborough Fault Zone (MFZ) (Walcott, 1998). The southern Alpine Fault continues offshore and merges with the Puysegur Trench (Figure 1.1c; Delteil et al., 1996; Lebrun et al., 2000.) Lebrun et al. (2000) interpreted the offshore extension of the Alpine Fault from Milford to George Sound in the northern Fiordland Margin from sea floor morphology. The offshore segment of the fault, inferred from bathymetric and seismic reflection, is geometrically more complex than the onshore segment (Barnes et al., 2005; Delteil et al., 1996). In the central Fiordland Margin, the Alpine Fault splits into the West branch linking the northern end of the Puysegur Trench, and the East branch. In the southern Fiordland Margin, the expression of the West and East branches can be seen from the sea floor morphology and seismic reflection data (Lebrun et al., 2000).

\subsection{Recent seismicity in the South Island of New Zealand}

As a result of ongoing collision between the Pacific and Australian tectonic plates, New Zealand is highly seismically active. This seismic activity has manifested itself as tens of thousands of $\mathrm{M}_{\mathrm{W}}>2$ reported earthquakes over the last five years. We briefly summarise the main characteristics of the most recent significant earthquakes in the South Island, namely the 15 July 2009 Dusky Sound, 4 September 2010 Darfield and 22 February 2011 Christchurch earthquakes and the Cook Strait earthquake sequence of July-August 2013. 
The largest earthquake to strike southern New Zealand since the $\mathrm{M}_{\mathrm{W}} 7.8$ Murchison earthquake of 1929 is the $\mathrm{M}_{\mathrm{W}} 7.8$ Dusky Sound earthquake (focal mechanism shown in Figure 1.4). Based on analysis of strong-motion records, Fry et al. (2010) inferred that rupture initiated at a depth of $38 \mathrm{~km}$ and proceeded upwards and southwestwards at a velocity of $\sim 2.6 \mathrm{~km} / \mathrm{s}$. Beavan et al. (2010a) modelled the fault geometry and slip distribution of the Dusky Sound earthquake using campaign and continuous Global Positioning System (GPS) data as well as ascending and descending tracks of the Advanced Land Observation Satellite (ALOS) (Figure 1.2a is a reproduction of Figure 8 from Beavan et al. 2010a). Their results suggest a maximum slip of $\sim 6 \mathrm{~m}$ occurring on a curve-shaped patch of approximately $4000 \mathrm{~km}^{2}$, equivalent to a scalar moment of $\sim 4.0 \times 10^{20} \mathrm{~N} \cdot \mathrm{m}$.

Both the $\mathrm{M}_{\mathrm{W}}$ 7.1 Darfield (Canterbury) earthquake of 2010 and the ensuing $\mathrm{M}_{\mathrm{W}} 6.2$ Christchurch earthquake of 2011, which caused 185 fatalities, occurred on previously unrecognised fault planes (focal mechanisms shown in Figure 1.4; Gledhill et al., 2011; Kaiser et al., 2012; Quigley et al., 2012). Gledhill et al. (2011) documented a complex source structure for the Darfield earthquake which seismic and geodetic observations suggest it initiated on a reverse fault and spread onto nearby strike-slip faults. The $\sim 30 \mathrm{~km}$ long surface rupture and the static stress drop of $\sim 13 \mathrm{MPa}$ of the Darfield earthquake were measured using the real-time kinematic and differential GPS and also airborne Light Detecting And Ranging (LiDAR) data by Quigley et al. (2012).

The Christchurch earthquake is notable for the high ground accelerations it produced near the epicentre; Fry et al. (2011) calculated a peak ground acceleration of 2 g. Coseismic ground displacements and slip distributions estimated by Beavan et al. (2012b) for the Darfield and Christchurch earthquakes are illustrated in Figure 1.2b and c; These figures are reproductions of Figure 2 from Beavan et al. (2012b).

The Cook Strait earthquake sequence of July-August 2013 began with two $\mathrm{M}_{\mathrm{W}} 5.5$ and 5.8 events, followed by a $M_{\mathrm{W}} 6.6$ event of 21 July 2013, off-shore from Seddon, and an onshore $\mathrm{M}_{\mathrm{W}} 6.6$ earthquake on 16 August 2013, near Lake Grassmere (focal mechanisms shown in Figure 1.4). Notable ground shaking was reported in Wellington and Marlborough with a peak ground acceleration of $0.2 \mathrm{~g}$ in parts of Wellington (Holden et al., 2013). Coseismic ground displacements and slip distributions associated with the $\mathrm{M}_{\mathrm{W}} 6.6$ earthquakes were measured by Hamling et al. (2014) using continuous and campaign GPS data along with two interferograms generated from TerraSAR-X (TSX) and RADARSAT-2 acquisitions (Figure 1.2d). Hamling et al. (2014) estimate predominantly dextral strike-slip of up to 0.9 and $2.1 \mathrm{~m}$ associated with the first and second events on the fault surface, respectively. Figure 1.2 is a reproduction of Figure 3 


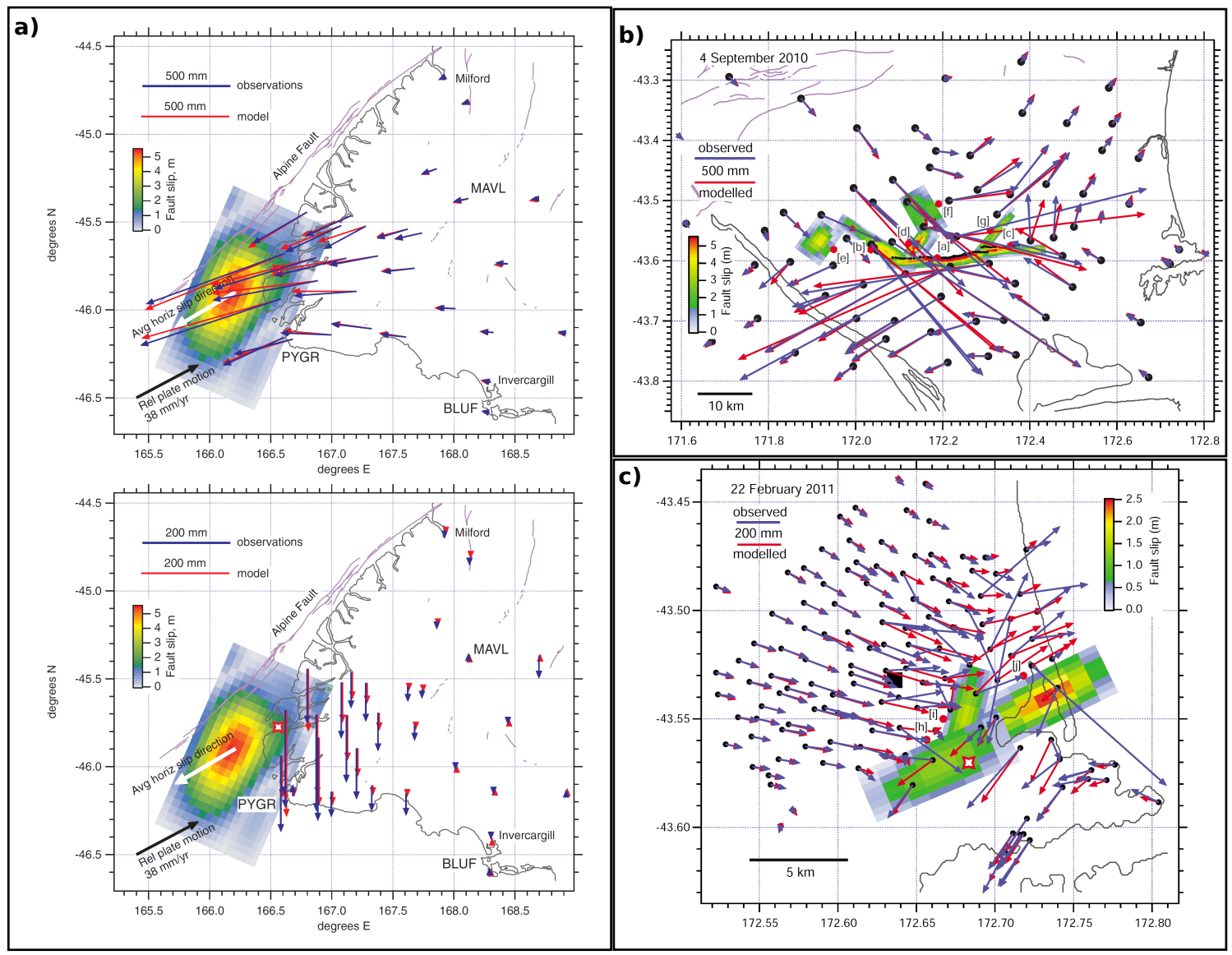

Figure 1.2: a) Coseismic slip distribution of the 15 July Dusky Sound earthquake, horizontal and vertical fits to the continuous and campaign GPS data acquired during August 2009 (Figure 8 from Beavan et al. 2010a). b) Coseismic slip distribution of the 4 September Darfield earthquake and horizontal fit to the continuous and campaign GPS data (Figure 2 from Beavan et al. 2012b). c) Coseismic slip distribution and horizontal fit to the continuous and campaign GPS data following the 22 February Christchurch earthquake (Figure 2 from Beavan et al. 2012b; continued on the next page).

from Hamling et al. (2014). We summarise the previous studies on the seismicity in the South Island schematically in this figure. Further details can be found in the papers by Beavan et al. (2010a, 2012b) and Hamling et al. (2014).

Other recent large earthquakes in the South Island include a $\mathrm{M}_{\mathrm{W}} 5.8$ earthquake northwest of Wanaka in the Otago region ${ }^{2}$ on 4 May 2015, a $\mathrm{M}_{\mathrm{W}} 6.0$ event west of Arthur's pass ${ }^{3}$ on 6 January 2015, a $\mathrm{M}_{\mathrm{W}} 6.2$ earthquake on 13 October 2014 in the Fiordland region ${ }^{4}$ and a $\mathrm{M}_{\mathrm{W}} 6.2$ earthquake on 24 April 2015, south-east of the Saint Arnaud Range ${ }^{5}$ in the northern South Island.

\footnotetext{
${ }^{2}$ http://geonet.org.nz/quakes/region/newzealand/2015p332712

${ }^{3}$ http://geonet.org.nz/quakes/region/newzealand/2015p012816

${ }^{4}$ http://geonet.org.nz/quakes/region/newzealand/2014p770859

${ }^{5}$ http://geonet.org.nz/quakes/region/newzealand/2014p770859
} 


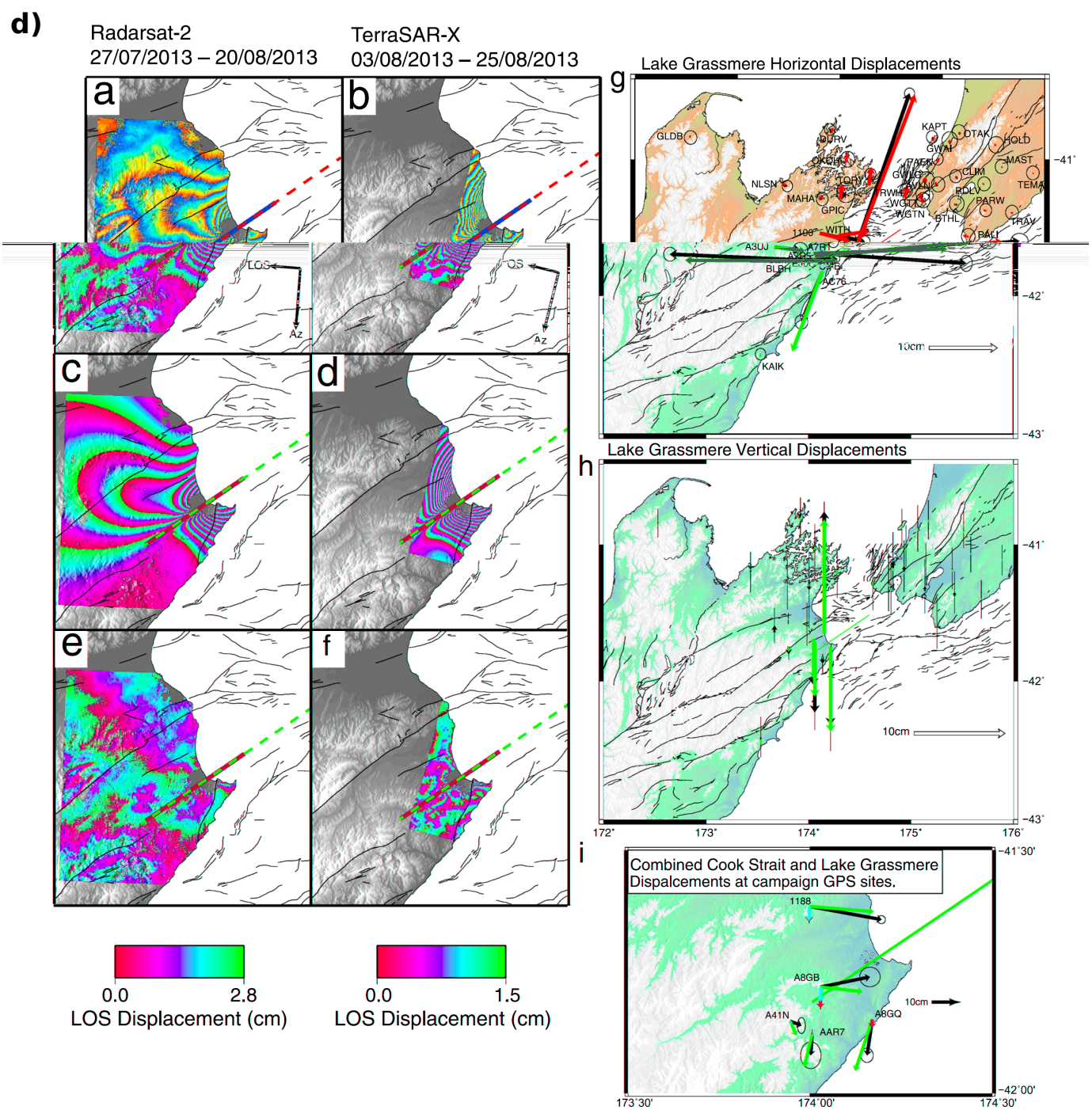

Figure 1.2: d) Observed and simulated RADARSAT-2 and TerraSAR-X interferograms for the Lake Grassmere earthquake (first and second columns). Observed (black) and modelled (red) horizontal and vertical GPS displacements due to the Lake Grassmere earthquake and sum of the horizontal displacements due the Cook Strait and Lake Grassmere earthquakes at five cGPS sites (third column), respectively (Figure 8 from Hamling et al. 2014). 


\subsection{Motivations and thesis objectives}

This project focuses on monitoring and modelling a range of fault-related processes that can be observed using geodetic means. The seismic and aseismic behaviours of faults encompass a wide range of processes with varying temporal and spatial scales (Tullis, 2007). Quasi-static earthquake nucleation, the propagation of dynamic rupture and its arrest, spatio-temporal variations in stress and fault strength and aseismic slip are all active at different places and stages in a fault system. During the phases related to nucleation and propagation of a rupture, stresses can change rapidly in a few seconds, with spatial variation of the order of a metre. Conversely, tectonic loading occurs over hundreds to thousands of years within the fault zone, on scales of kilometres. The major problem with modelling the entire process of the earthquake machine is dealing with this large range of scales. Therefore, as illustrated in Figure 1.3, this study addresses a specific range of spatial and temporal scales. In addition many complexities are encountered in modelling the earthquake cycle. Different assumptions of rheology, changes of stress field produced by aseismic slip, heterogeneity within the fault zone, thermomechanical coupling, spatial variability of constitutive law parameters, and offfault plasticity are some of the major sources of complexity during modelling. In this project, we only consider how different assumptions of rheology impact our modelling results.

We focus on the analysis and interpretation of geodetic data collected in the southern and central South Island of New Zealand during 2009-2014 and similar temporal and spatial scales highlighted in Figure 1.3. Our three primary research questions are stated below, with subordinate questions noted:

1. What can geodetic data reveal about postseismic displacement in response to multiple earthquakes?

(a) What circumstances or conditions are favourable in enhancing the effectiveness of Interferometric Synthetic Aperture Radar (InSAR) for measuring low-strain rate tectonic deformations?

2. What mathematical framework can we use to provide unique solutions of displacement and stress evolution for postseismic time intervals?

(a) How do we optimally choose rheology parameters in order to model the displacement and stress evolution?

(b) How can the uncertainty of input parameters for models such as fault geometry and slip distribution change the inferred stress changes? 


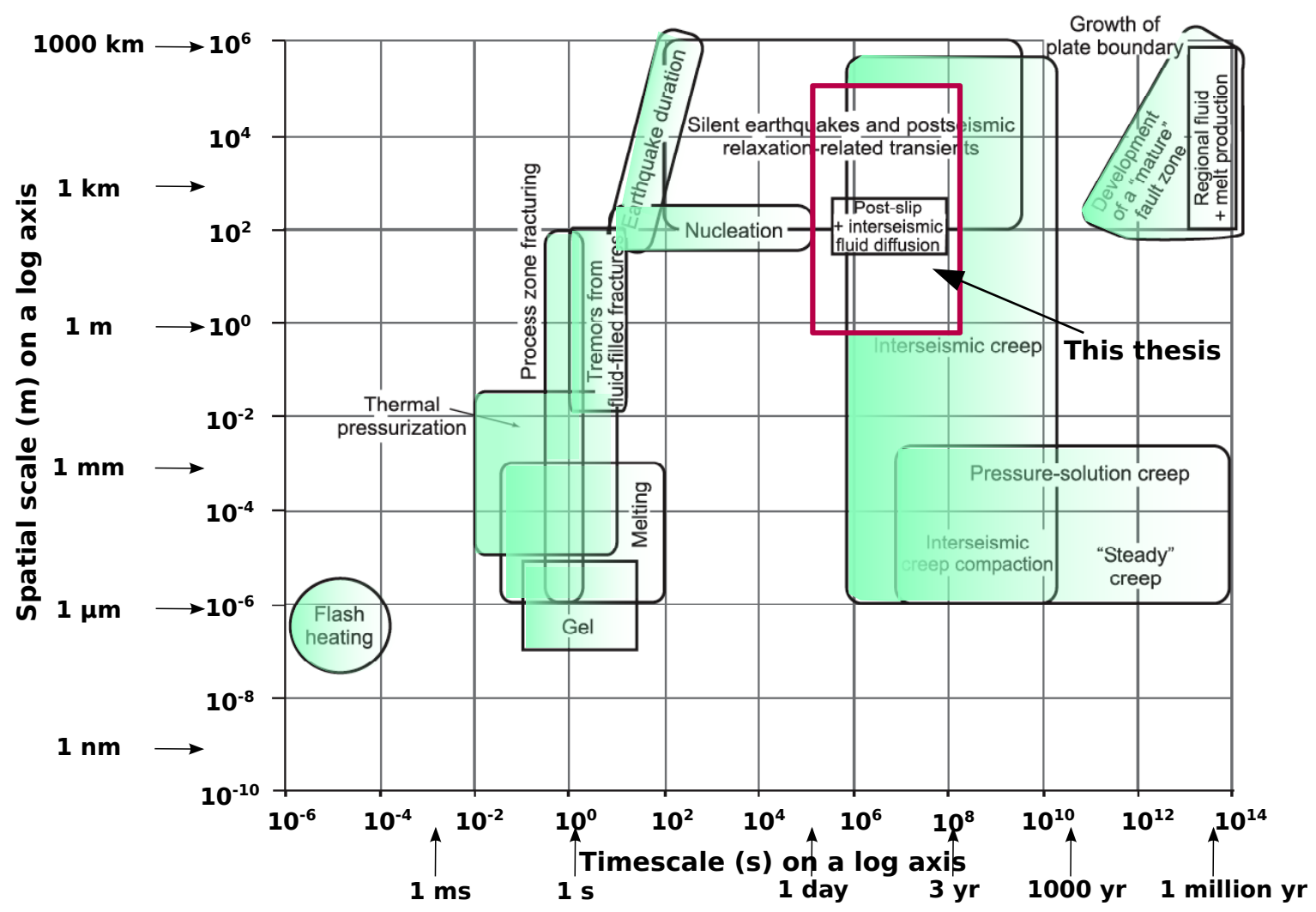

Figure 1.3: Temporal and spatial scales associated with various fault-related processes (modified from Tullis 2007). The processes of interest for this study (outlined in red in the figure) span temporal scales of days to several years and spatial scales from metres to hundreds of kilometres.

(c) Is the inferred model of stress evolution unique?

3. Can we assess the triggering effect of each earthquake on the later events by modelling the stress and strain evolution? Particularly, can we calculate the strain and stress evolution following the Dusky Sound earthquake and examine its effects on the Canterbury earthquake sequence of 2010-2012?

\subsection{Thesis outline}

The remainder of this thesis is composed of three stand-alone chapters as well as methodology and summary chapters. The three primary Chapters 3,4 and 5 are written for the purpose of subsequent publications. Therefore, for consistency, the first person plural ("we") has been used throughout the thesis and in some places contains duplication of background and introduction material. The regions of study for the stand-alone chapters are shown with boxes in Figure 1.4.

Most of the work in this thesis has been undertaken by myself (Pegah Faegh-Lashgary) 
with my supervisors' guidance unless explicitly stated. The titles and contents of the chapters are:

\section{Chapter 2: Measuring, modelling and interpreting postseismic displacement}

We first present an overview of contemporary space-geodesy techniques including satellite radar interferometry, known as InSAR and GPS with which to measure ground displacements associated with tectonic changes. This chapter next details basic theorems used to describe postseismic displacements in terms of 1) Viscoelastic relaxation; 2) Frictional afterslip; 3) Poroelastic rebound and 4) Dilatancy recovery. The governing equations for simple geometries and homogeneous materials for different mechanisms are discussed in analytical forms. The final part of the chapter describes semi-analytical approaches using propagator matrix methods and Finite Element Methods for fully numerical computation of stress-displacement vectors.

\section{Chapter 3: Postseismic displacement following the $M_{W} 7.8$ Dusky Sound earthquake, Fiordland, New Zealand, and its effect on the $M_{W} 7.1$ Darfield (Canterbury) earthquake 14 months later}

We describe the tectonic setting of southern New Zealand in the transition from the strike-slip Alpine Fault to the subduction interface of the Puysegur Trench in the Fiordland region (Figure 1.4). The postseismic displacement, as measured by continuous GPS in the time interval between the Dusky Sound and Darfield earthquakes along with campaign GPS data acquired during August 2009 and Differential Interferometric Synthetic Aperture Radar (DInSAR) observations, is modelled using an iterative approach. Prof. Euan Smith processed the continuous GPS data. The campaign GPS data acquired during August 2009 was published by Beavan et al. (2010a). We take advantage of the Principal Component Analysis-based Inversion Method (PCAIM) by which time-dependent slip models are estimated by decomposition of the postseismic displacement into space and time functions. Different values of viscosity yield different afterslip models for the fault embedded in an elastic-viscoelastic medium. Strain and Coulomb stress changes in the vicinity of the Darfield earthquake are calculated and discussed in terms of further effects on the Canterbury earthquake sequence of 2010-2012. Chapter 3 has been submitted to Geophysical Journal International in 
collaboration with John Townend, Charles Williams and Euan Smith.

\section{Chapter 4: Coupling time-dependent afterslip with viscoelastic rebound using analytical and hybrid numerical Green's func- tions}

We introduce a new inversion framework with which to split the postseismic signal into the viscoelastic responses to the coseismic and postseismic stress changes and afterslip. The novelty of the inversion technique is accounting for the corresponding viscoelastic responses to coseismic event and time-dependent afterslip. We develop the Principal Component Analysis-based Inversion Method used in Chapter 3 (Kositsky and Avouac, 2010) to form the Iterative Decoupling of Afterslip and Viscoelastic relaxation (IDAV) method in Chapter 4. In contrast with the conventional PCAIM inversion method, we use elastic and time-dependent elasto-viscoelastic Green's functions rather than instantaneous elastic Green's functions in order to relate a slip vector into displacement terms. The new approach (IDAV) is examined with analytically and numerically simulated displacement fields associated with a simple elastic-viscoelastic medium and an arbitrary geometry representing a subduction earthquake, respectively. We also apply the IDAV method to the observed postseismic displacement following the Dusky Sound earthquake. Chapter 4 has been submitted to Computers and Geosciences in collaboration with John Townend and Charles Williams.

\section{Chapter 5: Observations and modelling of postseismic displace- ment in Canterbury, New Zealand, following the 4 September 2010 Darfield and 22 February 2011 Christchurch earthquakes}

We explain the principal ground displacement features observed following the Darfield and Christchurch earthquakes in terms of poroelastic rebound, dilatancy recovery and afterslip. Dr. Sigrún Hreinsdóttir processed the campaign GPS data acquired annually from 2012 to 2015 . The afterslip models involve potentially a subsurface lineament manifested as a band of seismicity, the western faults, associated with the Darfield earthquake, and the eastern faults, associated with the Christchurch earthquake as well as its aftershocks. Chapter 5 has been submitted to Geophysical Journal International in collaboration with John Townend, Ian Hamling, Mahdi Motagh, Sigrún Hreinsdóttir and Paul Denys. 


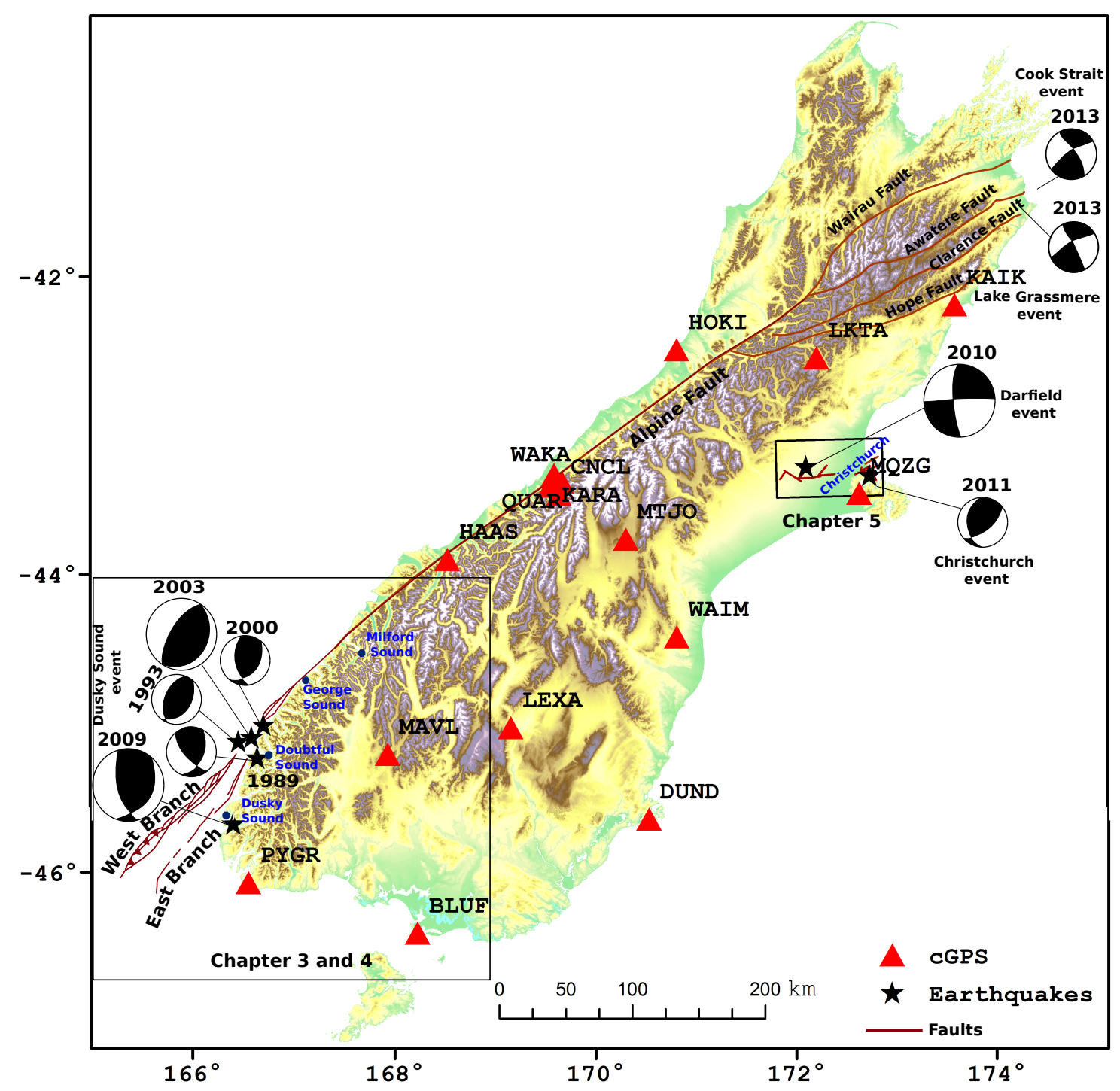

Figure 1.4: The Fiordland region: case area of Chapter 3 and 4. The Canterbury region: case area of Chapter 5. Focal mechanisms of recent earthquakes in the Fiordland region, namely the 1989 Doubtful Sound, 1993 Secretary Island, 2000 Thompson Sound, 2003 Fiordland and 2009 Dusky Sound earthquakes, in the Canterbury region, namely the 2010 Darfield and 2011 Christchurch earthquakes and the Cook Strait earthquake sequence which were widely felt in South Island of New Zealand. 


\section{Chapter 6: Summary and recommendations}

\section{Appendix A: Coulomb stress calculation}

Appendix A explains the transformation of a stress tensor from the Cartesian coordinate system to shear, normal and Coulomb components of stress changes. For our Coulomb stress calculation in Chapter 3, we assume an isotropic poroelastic rheology for the frictional model (Harris, 1998) with Skempton's and frictional coefficients of 0.5 and 0.6 , respectively.

\section{Appendix B: Global compilation of viscosity, rheology and depth of the top viscoelastic layer}

In Chapter 2 and Appendix B, we review different viscoelastic rebound models with a broad range of rheology and viscosity values with which other groups have modelled postseismic signals observed globally. In Appendix B, we summarise the region of study used in 88 papers and metadata of different earthquakes, as well as different assumptions about rheology, vertical or lateral heterogeneities and the viscosity for each layer. This appendix is referred to in detail in Chapter 2.

\section{Appendix C: Post-Dusky Sound supplementary files}

We illustrate fits to the post-Dusky Sound cGPS displacement time series with a viscosity of $2 \times 10^{18} \mathrm{~Pa} \cdot \mathrm{s}$ modelled by the IDAV method.

\section{Appendix D: Post-Canterbury supplementary files}

We present coseismic displacements associated with the 13 June and 23 December 2011 earthquakes in Christchurch and the standard deviation of the post-Christchurch mean Line Of Sight (LOS) velocity acquired from TSX images. 


\section{Chapter 2}

\section{Measuring and modelling postseismic displacement}

This chapter has two major sections, addressing the measuring and modelling of postseismic displacement. The detailed mathematical background and methods discussed in these sections compliment the stand-alone result chapters that follow (Chapters 3, 4 and 5). The first section describes the fundamentals of satellite-based geodetic techniques, including Interferometric Synthetic Aperture Radar (InSAR) and the Global Positioning System (GPS) that can be used to measure postseismic displacement. Secondly we discuss analytical and numerical models of observed displacements occurred on various temporal and spatial scales.

\subsection{Measuring postseismic displacement}

\subsubsection{Interferometric Synthetic Aperture Radar (InSAR)}

InSAR is a remote sensing technique used to measure displacement of the ground surface. Centimetre-scale movements can be inferred from measurements of the range change between the ground and antenna by differencing the phase from two radar images acquired at different times (e.g. Massonnet et al., 1993; Bamler and Hartl, 1998; Bürgmann et al., 2000; Rosen et al., 2000). The distance between two different sensors or the same sensor at different times is referred to as the baseline (Ferretti et al., 2007). The geometry of imagery for a pixel acquired by master and slave sensors with a baseline distance $B$ is illustrated in Figure 2.1. The variation of phase difference in this geometry from a ground reference point to its neighbour is approximately

$$
\Delta \phi=\frac{2 \pi \Delta r}{\lambda}=\frac{4 \pi}{\lambda} \frac{B_{\perp} q_{s}}{R}
$$

where

$$
\Delta r=\Delta\left(\Delta r_{0}\right)
$$




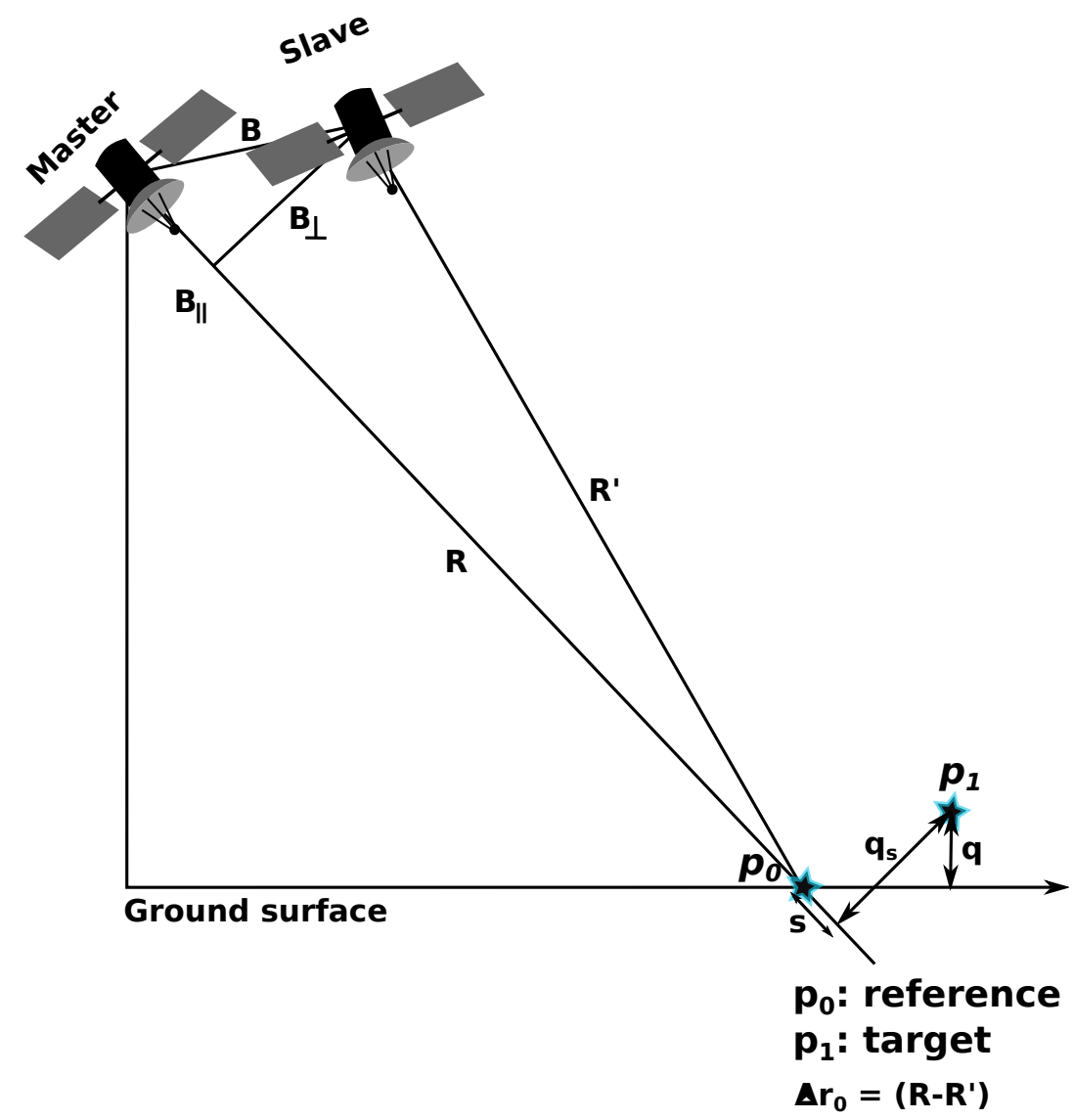

Figure 2.1: Schematic illustration of interferometric geomtry. All measurements are projected on the slant range. The distance between master and slave sensors is referred to as baseline with two perpendicular and parallel components $\mathrm{B}_{\perp}$ and $\mathrm{B}_{\|}$. For each target the perpendicular distance of the target to the slant range is $q_{s}$ and the projected distance on the slant range is $s$ (Ferretti et al., 2007).

The variation of phase difference $\Delta \phi$ is proportional to the variation of path length difference $\Delta r$ acquired from two Synthetic Aperture Radar (SAR) sensors between two points ( $p_{0}$; reference and $p_{1}$; target). $\Delta r_{0}$ is the reference path length difference acquired from master and slave sensors. $\lambda$ is the wavelength of the signal and $B_{\perp}$ is the perpendicular component of the baseline. All the measurements are projected to the slant range shown by $R$ in Figure 2.1. The altitude of the target, perpendicular distance from the slant range and slant range distance are represented by variables $q$, $q_{s}$ and $s$, respectively. The temporal baseline is the time difference between two sensor acquisitions. Temporal $\left(B_{\text {temp }}\right)$ and perpendicular $\left(B_{\perp}\right)$ baselines are the primary factors controlling the accuracy of InSAR processing (Ferretti et al., 2007).

The phase difference variation in Figure 2.1 consists of two components (Ferretti et al., 2007)

$$
\Delta \phi=\Delta \phi_{\text {topo }}+\Delta \phi_{\text {geom }}
$$


where

$$
\Delta \phi_{\text {topo }}=-\frac{4 \pi}{\lambda} \frac{B_{\perp} q}{R \sin \theta}
$$

and

$$
\Delta \phi_{\text {geom }}=-\frac{4 \pi}{\lambda} \frac{B_{\perp} s}{R \tan \theta}
$$

The example shown in Figure 2.1 has a simple interferometric geometry in which we assume there is only one dominant point scatterer within a pixel. Multiple scatterers within a pixel contribute to the phase value of the pixel (Ferretti et al., 2000, 2001). The multiple contributions impose randomness in the phase value of a Single Look Complex (SLC). By subtracting the phase value from two SLCs acquired at two epochs, this random contribution is removed, but it is subject to unchanged backscattering characteristics of the points within the pixel between two epochs (Ferretti et al., 2000, 2001). Thereafter, the differences between the atmospheric path delay in two epochs $\phi_{a t m}$, displacement $\phi_{\text {def }}$, and noise $\phi_{\text {noise }}$ appear on the interferometric phase in addition to the differences between viewing geometry $\phi_{\text {geom }}$ and topography $\phi_{\text {topo }}($ Ferretti et al., 2000, 2001). The interferometric phase then is written as

$$
\Delta \phi=\left(\Delta \phi_{\text {topo }}+\Delta \phi_{\text {geom }}\right)+\left(\Delta \phi_{\text {atm }}+\Delta \phi_{\text {def }}+\Delta \phi_{\text {noise }}\right)
$$

and

$$
\Delta \phi_{\text {def }}=\frac{4 \pi}{\lambda} d
$$

Figure 2.2 illustrates how the radar interferometric phase changes with ground displacement $d$. Two SAR images acquired prior to and following a displacement-inducing event measure the phase difference between the two epochs, which is converted to ground displacement using Equation 2.7. However, as shown in Equation 2.6, the phase difference consists of other components in addition to the displacement phase. The interferometric geometry in which the data are acquired, the topography of the ground and atmospheric and orbital errors introduce other components to the phase difference that we must remove to isolate the displacement phase (see Bürgmann et al., 2000; Elachi and Van Zyl, 2006; Ferretti et al., 2007; Dzurisin and Mori, 2007). We remove components related to geometry and topography during InSAR processing. We calculate the effects of viewing geometry using orbital data during the step known as interferogram flattening (Figure 2.3a). The topographic phase is calculated using either a Digital Elevation Model (DEM) spanning the entire region (two-pass differential interferometry) or utilising a pair of images acquired before the event (three-pass differential interferometry; Figure 2.3b). We can use the DEM product of the Shuttle Radar Topography Mission (SRTM) for two pass differential interferometry. The effects of 


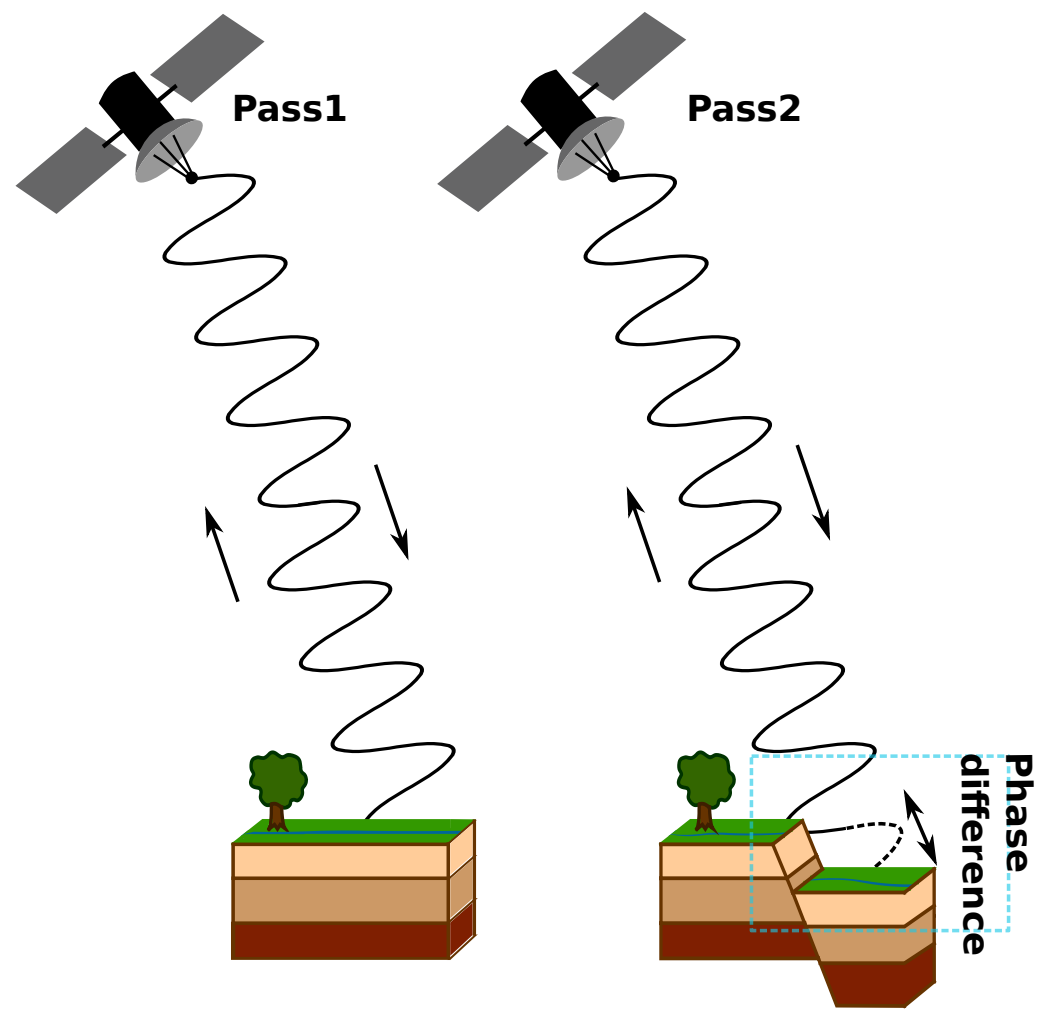

Figure 2.2: The principle of phase measurement due to a surface displacement in radar interferometry.

atmospheric and orbital errors on the displacement signal are mitigated by applying several post-processing methods (Figure 2.3c; see Bürgmann et al., 2000; Elachi and Van Zyl, 2006; Ferretti et al., 2007; Dzurisin and Mori, 2007).

\section{InSAR time series techniques}

InSAR has a unique advantage over other geodetic methods in its ability to measure surface displacement with millimetre- to centimetre-scale accuracy over large areas (on the order of hundreds of square kilometres; e.g. Massonnet et al., 1993; Bamler and Hartl, 1998; Bürgmann et al., 2000; Rosen et al., 2000). Temporal and baseline decorrelation and variations due to atmospheric conditions are major limitations of conventional InSAR techniques (Bürgmann et al., 2000). The effect of decorrelation is reduced by combining multiple SAR observations using multi-temporal processing techniques. Using more than two SAR scenes acquired over the same area leads to redundant measurements that are used with time series methods to produce crustal displacement maps (Ferretti et al., 2001; Kampes, 2005; Berardino et al., 2002).

InSAR time-series techniques are broadly classified into two categories: 1) Permanent Scatterer-InSAR (PS-InSAR); and 2) Small BAseline Subset (SBAS) methods. 
a)

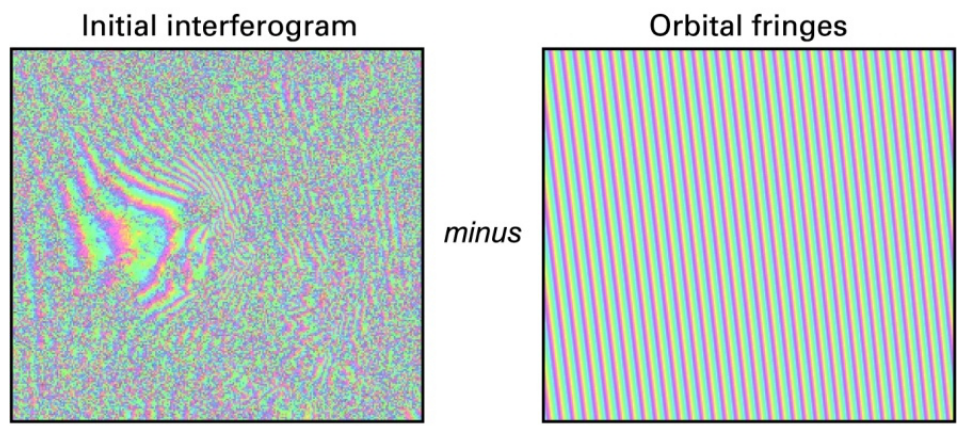

Remove viewing geometry (orbits and imaging geometry)

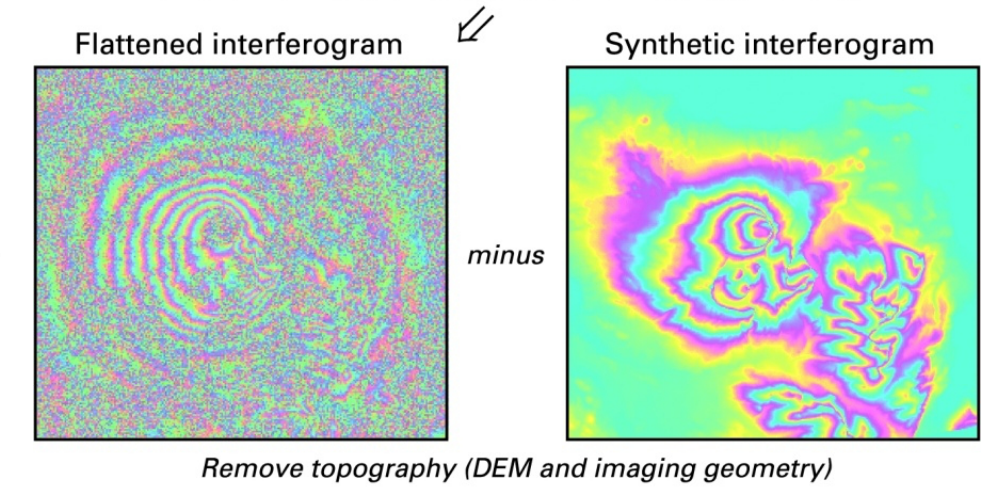

Topography-removed interferogram

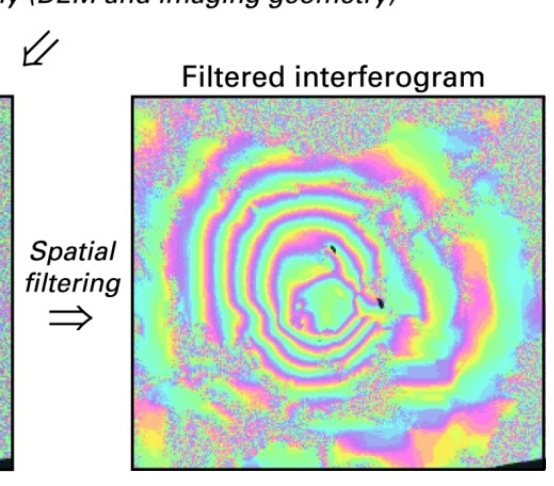

Figure 2.3: Interferometry workflow (Dzurisin and Mori, 2007). a) Interferogram flattening b) Differential interferometry c) Spatial filtering. Each fringe in the filtered interferogram represents displacement of the ground surface towards the satellite by an amount equal to half of the wavelength. 
PS-InSAR works by identifying image pixels in a stack of interferograms that remain coherent over long time intervals, with little variation of their scattering properties with either imaging geometry or time (Ferretti et al., 2000; Hooper et al., 2004). The first implementation of PS-InSAR worked well in urban areas where man-made structures behave like strong corner reflectors, but fails to perform satisfactorily in vegetated and mountainous terrains (Ferretti et al., 2000, 2001). The Stanford Method for Permanent Scatterers (StaMPS) technique, developed by Hooper et al. (2004) and modified by Hooper (2006), was the first PS-InSAR method designed to estimate displacement signals even in the absence of very bright scatterers, using Slowly Decorrelating Filtered Phase (SDFP) pixels. Filtered phase values of SDFP pixels decorrelate very little over short time intervals. This method was initially used to analyse episodic crustal displacements in non-urban environments, with application to volcanic settings. The SBAS technique (Berardino et al., 2002) minimises the effects of decorrelation on displacement signals by constructing all possible interferograms in the study area with short temporal and spatial baselines. Using SBAS techniques, velocity maps and time series of coherent pixels are produced (Lanari et al., 2007). The time-series analysis in the SBAS method is performed using the least-squares inversion methodology.

\section{Examples of InSAR time series}

Here we present examples of ground displacements in Christchurch prior to and following the $\mathrm{M}_{\mathrm{W}}$ 7.1 Darfield earthquake on 4 September 2010. This is done using Envisat and CSK acquisitions and PS-InSAR method combined with SBAS. Note that, these results are not used in other chapters for further modelling.

We measure the ground displacement for the first dataset using satellite radar interferometry including 36 Envisat SAR acquisitions in ascending orbits from February 2004 to July 2010. The second dataset spans the time interval between October 2010 and February 2011 and includes 12 CSK acquisitions in ascending orbits. We process both datasets in the same way. After reading and cropping the SLCs, we extract the satellite orbits for all images and apply DEM offset corrections using the LINZ $40 \mathrm{~m}$ DEM. All interferograms for each dataset are coregistered and resampled and all master-slave interferograms are produced using the DORIS software package (Kampes and Usai, 1999). The SBAS networks corresponding to the Envisat and CSK datasets consist of 38 and 117 interferograms, respectively. For the Envisat dataset, PS pixels are selected using the default values of PS-selection and weeding parameters of StaMPS (Hooper and Zebker, 2007; Hooper, 2008; Hooper et al., 2010). For the CSK dataset, pixels

\footnotetext{
${ }^{1}$ Land Information New Zealand; https://data.linz.govt.nz
} 
with coherence above a threshold of 0.2 are selected as Permanent Scatterer (PS). After 3D phase unwrapping the times series of displacement and the associated velocity are estimated using StaMPS (Hooper and Zebker, 2007; Hooper, 2008; Hooper et al., $2010)$.

Figure 2.4 (the Envisat dataset) shows a subsidence signal over western Christchurch that occurred over a long period of almost 6 years prior to the Canterbury sequence of 2010-2012. The subsidence occurs immediately west of the transition from confined to unconfined aquifers, in an area where the water table is locally deep (exceeding 6 $\mathrm{m}$ ), unconfined groundwater is downwards flowing and levels are highly variable (Cox et al., 2012; Van Ballegooy et al., 2013; Figure 2.4a and b).

Figure 2.5 (the CSK dataset) represents accumulated displacement due to a number of aftershocks (red stars) particularly on the December 26th, 2010, which impacted the Central City (Bannister and Gledhill, 2012). Several aftershocks shown in Figure 2.5 have magnitudes larger than $M_{w}$ 4.0. These results demonstrate how SBAS and PS-InSAR time series technique capture small magnitudes of displacements in Christchurch. 

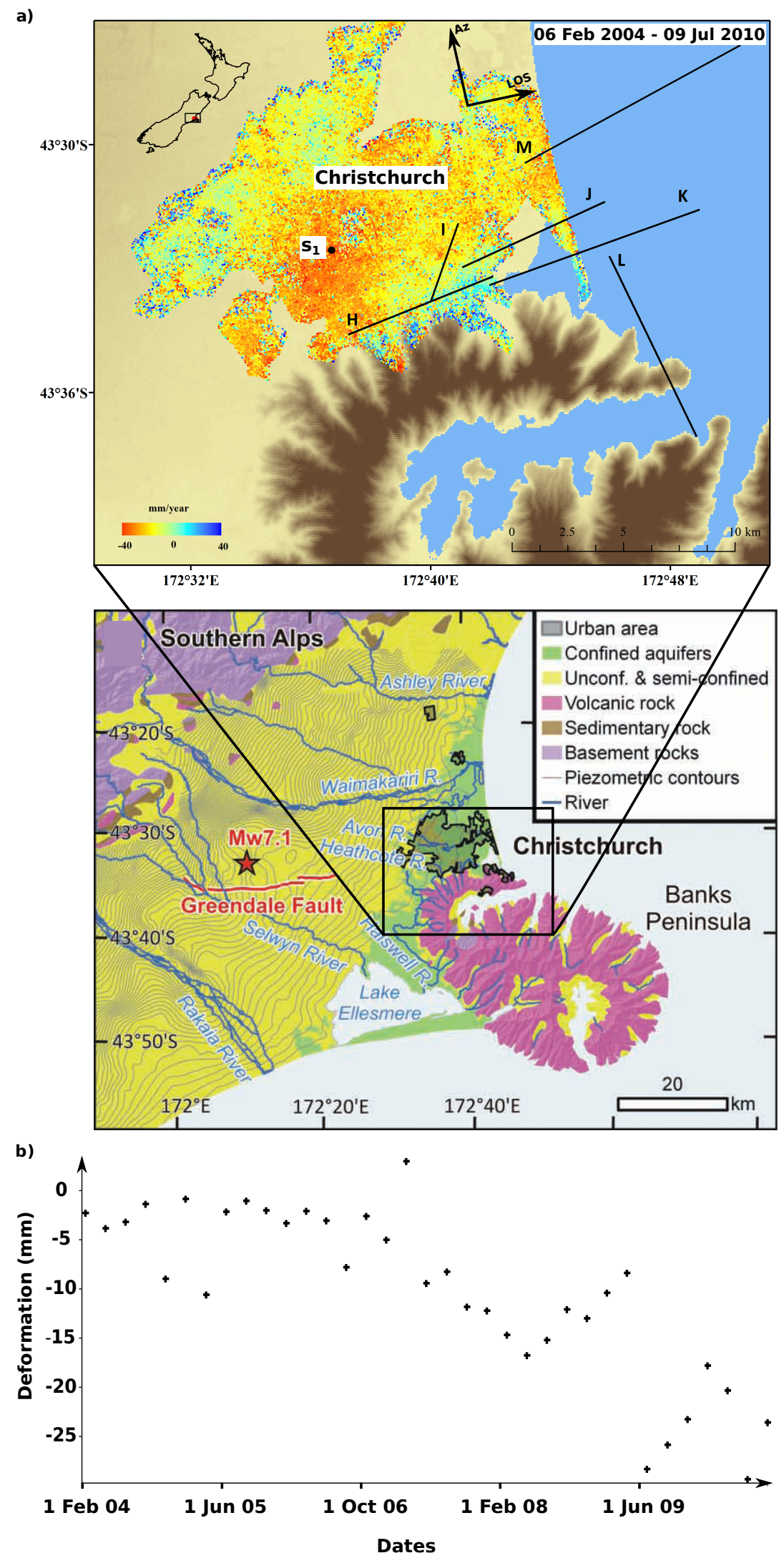

Figure 2.4: a) Mean line of sight velocity measured from ASAR/Envisat acquisitions prior to the 4 September 2010 Darfield earthquake. The colors represent displacement rates in the LOS directions: red colors indicate movement away from satellite and blue colors indicate movement towards the satellite. Transition from confined to unconfined acquifers is inferred from Cox et al. (2012) b) Time series of displacement at point $S_{1}$ in Christchurch extracted from the Envisat dataset. Faults are as described in Figure 2.5. 


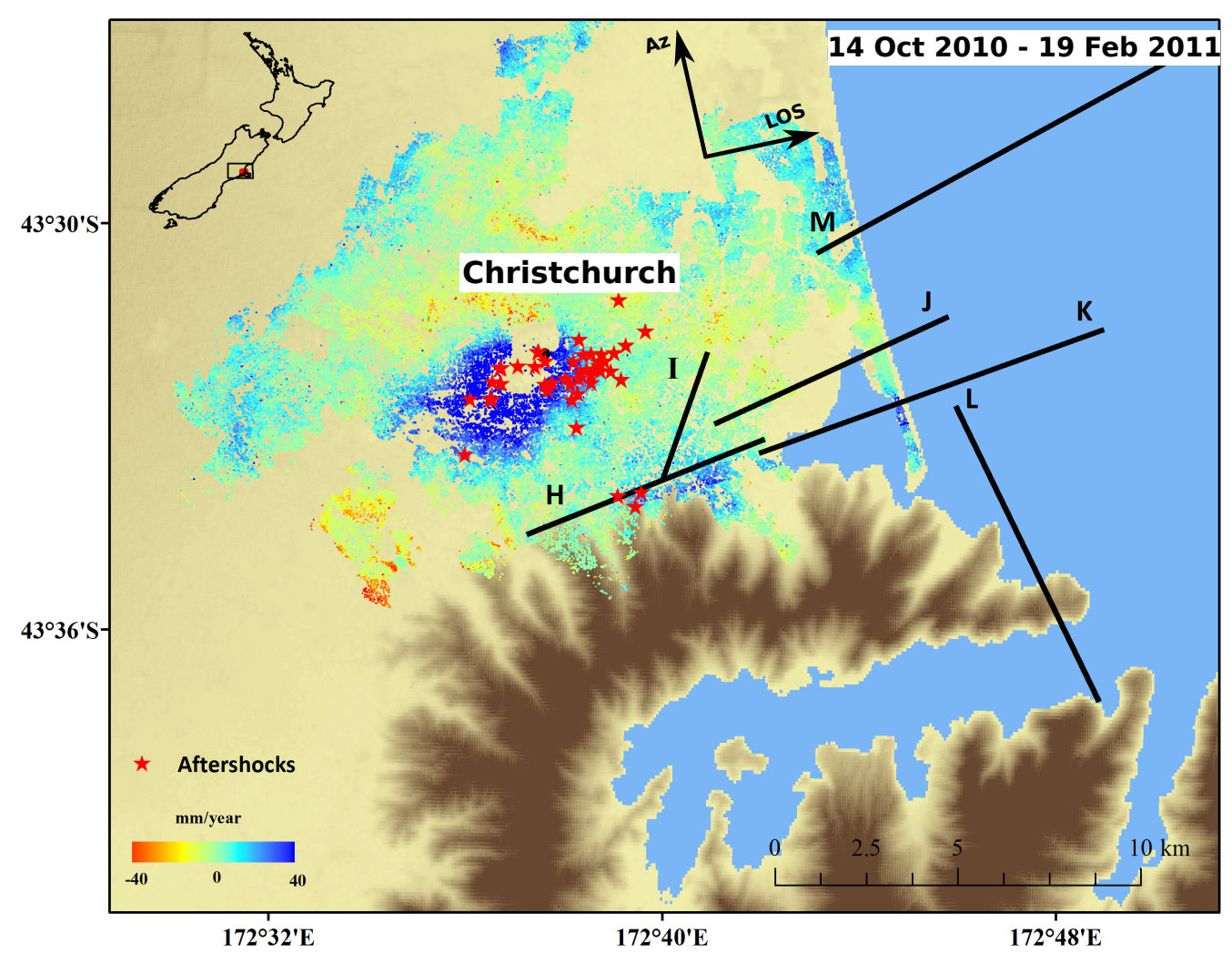

Figure 2.5: Mean line of sight velocity measured from COSMO-SkyMed ascending orbits following the Darfield and prior to the Christchurch earthquakes. Red stars represent aftershocks between October 2010 and February 2011. For permanent scatterers, the colours represent displacement rates in the LOS direction: red indicates movement away from satellite and blue indicates movement towards the satellite. H) Western ENE I) NNE J) Eastern faults related to the 22 February Christchurch earthquake, K) SW-NE L) NW-SE Faults related to the 13 June 2011 aftershock and M) SW-NE Fault related to the 23 December 2011 aftershock.

\subsubsection{Global Positioning System (GPS)}

In GPS techniques, distances between satellites and receivers are measured to compute point positions on the Earth surface. Similar to trilateration, GPS surveys require known positions of control points (satellite positions). The position of the receiver in the Cartesian coordinate system $\left(u_{x}, u_{y}, u_{z}\right)$ and the receiver's clock offset $d T_{u}$ are four unknown parameters that are estimated from measuring distances between receiver and a minimum of four other satellites (see Remondi, 1985a,b, 1988, 1991; Figure 2.6a).

The two observable variables in GPS surveys are the pseudo-range for lower-accuracy and the carrier phase for high-precision surveys. Pseudo-range $p$ and carrier phase $\phi$ 

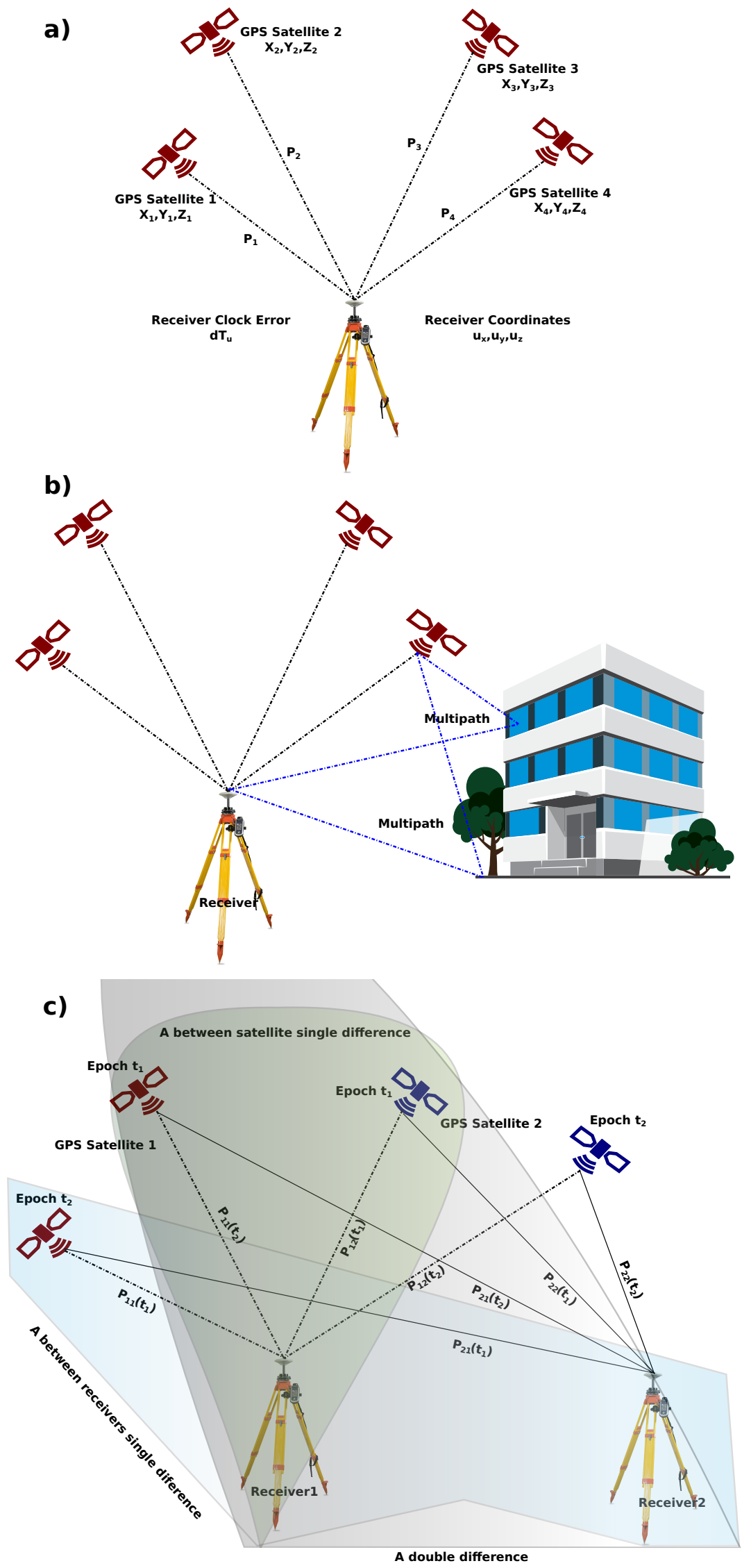

Figure 2.6: a) A navigation solution for GPS measurements of the position and receiver clock with a minimum of four other satellites b) Multipath is highlited by blue dashed lines c) Techniques of single between-receivers indicated by pale blue area, between-satellites by pale green are, double difference by pale pink area and triple difference by the overall area (Van Sickle, 2008). 
observations for each satellite-receiver pair with the distance of $\rho$ are

$$
\begin{aligned}
& p=\rho+d_{\rho}+c(d t-d T)+d_{\text {ion }}+d_{\text {trop }}+\epsilon_{m_{p}}+\epsilon_{p} \\
& \phi=\rho+d_{\rho}+c(d t-d T)+\lambda N-d_{\text {ion }}+d_{\text {trop }}+\epsilon_{m_{\phi}}+\epsilon_{\text {phi }}
\end{aligned}
$$

respectively, where $N$ is the the number of full wavelengths $\lambda$ associated with the modulated carrier. These calculations include several biases in observation equations such as: orbital errors $d_{\rho}$, ionospheric $d_{i o n}$ and tropospheric effects $d_{\text {trop }}$, receiver noises $\epsilon_{p}, \epsilon_{\phi}$, multipath $\epsilon_{m_{p}}, \epsilon_{m_{\phi}}$ and satellite and receiver clock errors $(d t-d T) . \rho$ is pseudorange or carrier phase value excluding the biases in observations.

A satellite clock error $d t$ is monitored by the Control Segment which broadcasts clock corrections as part of the navigation message. The navigation message includes all parameters required to perform a positioning service, which is sent back to the users (see Misra and Enge, 2006; Van Sickle, 2008). Effects of refraction and reflection on the direction and speed of the signal while it travels through atmosphere is another source of bias. These errors are larger for a signal travelling from satellites located near the receiver's horizon than its zenith, due to the amount of time that a signal interacts with the ionosphere while it travels from horizon (see Misra and Enge, 2006; Van Sickle, 2008).

The magnitude of ionospheric delay can be determined from the Total Electron Content (TEC) in the ionosphere, which has a daily cycle (see Coco, 1991; Borries et al., 2007). The ionospheric effect is dispersive, meaning that it is correlated with the frequency of the signal. This effect is therefore modelled and removed for dual-frequency receivers using L1 (1575.42 MHz) and L2 (1227.60 MHz) frequencies. For singlefrequency receivers, ionospheric model parameters in the navigation message are useful to partially (but not completely) remove ionospheric refraction effects.

Satellite positions are obtainable from broadcast ephemerides or final orbits generated by analysis centres a few weeks after the observation based on tracking satellites. A precise satellite position leads to a precise estimation of receiver position (e.g. Malys et al., 1997; Van Sickle, 2008). Associated errors in the estimation of satellite position remain as orbital errors $d_{\rho}$ in the final position measurement.

In contrast to the ionospheric effects, tropospheric errors are non-dispersive with similar effects on L1 and L2 frequencies. The pressure-correlated dry component contributes more to the total tropospheric errors and it is easier to be estimated. The wet component of the tropospheric effects requires information of water vapour content in the troposphere (see Dodson et al., 1996; Van Sickle, 2008).

Errors due to multipath occur when signals are reflected from nearby objects and inter- 
fere with the direct signal from the satellite (Figure 2.6b). Some receivers identify and reject weaker and more diffuse reflected signals or reverse circular polarization. Minimising the effect of multipath is also possible via GPS antenna design (see Van Sickle, 2008).

\section{Differencing}

Single, double and triple differences are the techniques most frequently used to mitigate or remove different position measurement biases. Satellite clock and orbital errors are assumed to be very similar in measurements from the same satellite but different receivers when using the technique called between-receiver single difference (Figure 2.6c, pale blue polygon). In a single between-satellite difference technique, receiver clock errors are eliminated by differencing simultaneous measurements from two satellites (Figure 2.6c, pale green polygon). Atmospheric effects also remain quite similar for satellites with a common receiver, so this technique somewhat mitigates the effects of ionospheric and tropospheric errors. With a double difference technique using a combination of both between-satellite and between-receiver difference techniques, we remove both satellite and receiver clock errors (Figure 2.6c, pale pink polygon). Two double difference techniques combined at two different epochs are referred to as a triple difference by which the integer cycle ambiguity $N$ (Equation 2.9) is quantifiable (see Parkinson and Enge, 1996; Misra and Enge, 2006; Van Sickle, 2008; Figure 2.6c).

\subsection{Modelling postseismic displacement}

Postseismic displacements following large earthquakes have generally been modelled using four mechanisms: 1) long-term viscoelastic relaxation in the lower crust/upper mantle (e.g. Elsasser, 1967; Savage and Prescott, 1978; Pollitz et al., 2000; Pollitz, 2003b; Rousset et al., 2012a); 2) short-term afterslip in the upper/lower crust on the mainshock fault surface (e.g. Perfettini and Avouac, 2007; Ryder et al., 2010b; Johnson et al., 2012; Lubis et al., 2013); 3) poroelastic rebound (e.g. Booker, 1974; Rice and Cleary, 1976; Peltzer et al., 1998; Wang and Kümpel, 2003; Hu et al., 2014); and 4) shallow fault zone dilatancy recovery (e.g. Fielding et al., 2009). We interpret the observed postseismic displacements following the 15 July 2009 Dusky Sound earthquake and the Canterbury earthquake sequence of 2010-2012 in terms of these four mechanisms. In the following sections, we focus on the principles employed to derive the governing equations for each postseismic mechanism. In order to derive analytical solutions for stress-displacement vectors, we often assume a simple geometry and homogeneous 
properties. The components of stress-displacement vectors are combinations of stress and displacement. We discuss forward analytical models in detail.

In addition, we need inversion techniques to interpret geodetic observations. Observations of time-dependent postseismic displacement using satellite-geodesy techniques enable us to analyse time-dependent mechanisms. In this chapter, we describe methods of time-dependent inversion of postseismic displacement. More details are presented in Chapters 3 and 4 of this thesis.

More realistic postseismic models require consideration of complex properties, such as an irregular non-planar medium (e.g. Pollitz, 1992), multiple relaxation time scales (Hetland and Hager, 2005), lateral heterogeneity (Pollitz, 2003a), vertical layering (Hetland and Hager, 2005) and the effects of coupling postseismic mechanisms with gravity (Pollitz, 1997; Rundle, 1980; Iwasaki, 1985). Deriving analytical solutions for complex problems is often impossible. Consequently, semi-analytical approaches and numerical models are extensively used to approximate complex mathematically-defined models (e.g. Haskell, 1953; Schapery, 1967; Peltier, 1985; Wang and Kümpel, 2003; Fukahata and Matsu'ura, 2006; Aagaard et al., 2009, 2013). We review the basic ideas behind the semi-analytical and numerical methods employed in this thesis. In particular, the Finite Element Method (FEM) using the numerical code PyLith (Aagaard et al., 2009, 2013, 2015) and the propagator matrix method of Fukahata and Matsu'ura (2006) are used to compute numerical and semi-analytical viscoelastic Green's functions in Chapters 3 and 4. In Chapter 5, we adopt the framework developed by Wang and Kümpel (2003) for computing the effects of multi-layered poroelastic rebound using an extension of Haskell's propagator method and the technique used by Fielding et al. (2009) for estimating shallow fault zone dilatancy recovery process following the Canterbury 2010-2012 earthquake sequence.

\subsubsection{Viscoelastic relaxation of ductile materials}

Viscoelastic effects during the postseismic time interval relax the deviatoric stress induced coseismically (e.g. Freed and Lin, 1998, 2001; Rydelek and Sacks, 2001; Freed, 2005; Pollitz et al., 2006a; Freed et al., 2007; He et al., 2011; Decriem and Árnadóttir, 2012).

The brittle-ductile transition of rocks occurs with increasing temperature at depths. The ductile behaviour of rocks in the lower crust and upper mantle leads to diffusion and dislocation creep processes (see Karato and Wu, 1993; Fliervoet et al., 1999; van Hunen et al., 2005). Mantle convection, which controls the cooling of the earth, is a result 
a)
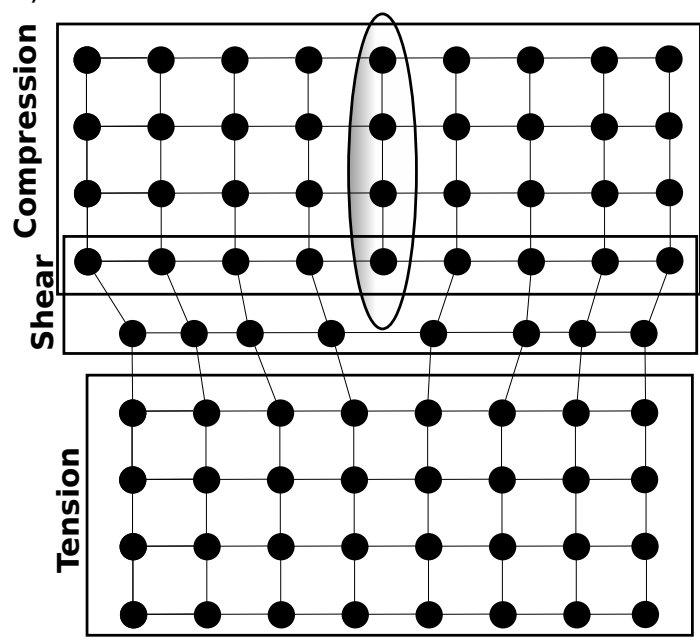

b)

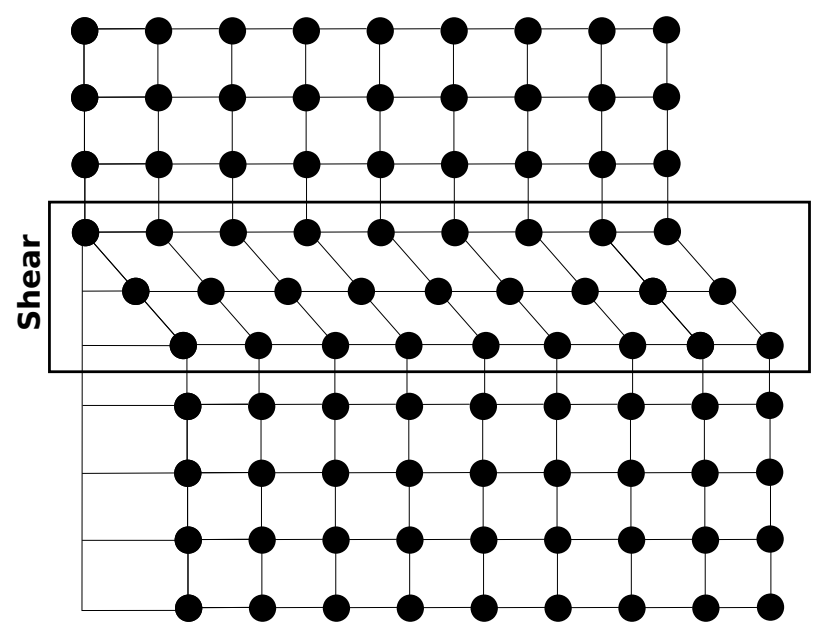

Figure 2.7: Schematic illustration of atomic displacement and lattice deformation from a) Edge dislocation creep. Compression, tension and shear zones are presented in boxes. An extra half-plane occurs in the compression zone. b) Purely shear screw dislocation creep.

of these processes. The creep processes also alter the stress induced by earthquakes, producing postseismic viscoelastic relaxation (see Turcotte and Schubert, 2014).

Creep processes are defined based on Fick's law (Fick, 1855). Atoms tend to move from high to low density, in order to reach the most stable state with evenly distributed vacancies in crystals. At lower stresses, diffusion creep dominates and rocks behave as a Newtonian fluid, with linear pressure- and temperature-dependent viscosities (Kundu and Cohen, 1990; Batchelor, 2000; Kirby, 2010). At higher stresses, edge and screw dislocations are major forms of dislocation creep that cause a power-law or non-Newtonian behaviour of fluids (Astarita and Marrucci, 1974). Dislocations cause atomic-scale deformations in crystals. Edge dislocations in crystals yield an extra halfplane in the lattice (Figure 2.7a, ellipse). Furthermore, edge dislocations produce a region of compression where the extra half-plane occurs, a tension field and a shear region (Figure. 2.7a). In contrast, screw dislocations cause an offset in the lattice and a purely shear dislocation (Figure. 2.7b) region in crystals. If the dislocation creep persists, the viscosity follows a stress-dependent power-law rheology. Laboratory studies of olivine, the primary mineral of the mantle, show that dislocation creep is the dominant process (see Karato et al., 1986; Karato and Wu, 1993; Turcotte and Schubert, 2014). However, mantle strain rates are much lower than laboratory strain rates (see Paterson, 1987; Turcotte and Schubert, 2014). It is not inferred from laboratory studies whether the dominant process is the dislocation creep, nor whether the mantle behaves as a non-Newtonian fluid (see Turcotte and Schubert, 2014). 
The general viscosity $\eta$ for either dislocation or diffusion creep is expressed as

$$
\eta=C\left(\frac{\sigma}{\mu}\right)^{1-n} \exp \left(\frac{E_{a}+p V_{a}}{R T}\right)
$$

where

$$
C=\frac{1}{2}\left(\frac{\mu}{A}\right)\left(\frac{h}{b}\right)^{m}
$$

Here $C$ is the pre-exponential factor, $n$ is a power, $\mu$ is the shear modulus, $\sigma$ is the stress magnitude, $E_{a}$ and $V_{a}$ are the activation energy and activation volume per mole, $R$ is the universal gas constant and $p$ and $T$ are pressure and absolute temperature (Kirby and Kronenberg, 1987; Carter and Tsenn, 1987). In Equation 2.11, $b$ and $h$ are the lattice spacing and grain size, respectively, $m$ is a power and $A$ is a constant. For diffusion creep, $n=1$ and $m=2.5$ are appropriate powers. In the particular case where dislocation creep is independent of the grain size and the lattice spacing, $m=0$ and $n=3.5$. We infer from Equation 2.10 that regardless of the dominant creep mechanism, the viscosity is exponentially proportional to the pressure and inverse of absolute temperature. Furthermore, if $n \neq 1$ the equation implies stress dependent viscosity for the mechanism of dominant dislocation creep (see Turcotte and Schubert, 2014).

Elsasser (1969) considered the viscous behaviour of the asthenosphere due to diffusion and dislocation creep processes with a simple one-dimensional model, assuming an elastic layer overlying a viscous channel. In this model, the elastic behaviour of the asthenosphere at short time scales was not considered. Several researchers have since attempted to model the viscoelastic relaxation of ductile materials, considering the elastic behaviour of the asthenosohere, with an elastic layer overlying a viscoelastic halfspace. This two-layer model is the most widely used representation of the brittle-ductile transition (e.g. Flügge, 1975; Iwasaki, 1985; Pollitz, 1997; Hearn et al., 2002b; Hu et al., 2004; Pollitz, 2003a; Fukahata and Matsu'ura, 2006). We use this representation in Chapters 3 and 4, where we only consider linear viscoelastic materials (Newtonian fluids) for the viscoelastic half-space with dominant diffusion creep, and $n=1$.

\section{Correspondence Principle}

We use the Correspondence Principle to achieve analytical solutions to viscoelastic problems (e.g Nur and Mavko, 1974; Savage and Prescott, 1978). Stresses arising from a Newtonian fluid flow are linearly proportional to the strain rate (Kundu and Cohen, 1990; Batchelor, 2000; Kirby, 2010). The Laplace transform of this linear constitutive law is expressed as a linear relation between transformed stress and strain. Conse- 
quently, the governing equations of viscoelasticity after applying the Laplace transform, become similar to the corresponding elastic governing equations. This statement is known as the Correspondence Principle. The derivation of this principle is as follows The constitutive law between shear stress $\sigma$ and shear strain $\epsilon$ for linear viscoelastic media is expressed as

$$
\Phi \sigma=\Psi \epsilon
$$

Depending on the rheology of the media

$$
\Phi=\sum_{k=0}^{q_{\phi}} \phi_{k} \frac{d^{k}}{d t^{k}}
$$

and

$$
\Psi=\sum_{k=0}^{q_{\psi}} \psi_{k} \frac{d^{k}}{d t^{k}}
$$

where $\phi_{k}$ and $\psi_{k}$ are the stress and strain coefficients and determined by their rheological properties (Flügge, 1975; Findley et al., 1977). The Laplace transform of equations 2.13 and 2.14 reveals the Correspondence Principle

$$
\begin{aligned}
& \hat{\Phi}=\hat{\Phi}(s)=\sum_{k=0}^{q_{\phi}} \phi_{k} s^{k} \\
& \hat{\Psi}=\hat{\Psi}(s)=\sum_{k=0}^{q_{\psi}} \psi_{k} s^{k}
\end{aligned}
$$

where the Laplace transform of a function $f(t)$ is the function $F(s)$

$$
F(s)=\int_{0}^{\infty} \exp (-s t) f(t) d t
$$

The time derivatives of equations 2.13 and 2.14 are transformed to linear combinations of the Laplacian variable $s$ in equations 2.15 and 2.16 .

\section{Rheology of the viscoelastic half-space}

The stress and strain coefficients $\phi_{k}$ and $\psi_{k}$ in Equations 2.13 and 2.14 are defined by the rheology of the viscoelastic half-space (see Hetland and Hager, 2005; Segall, 2010). The Newtonian rheology of the viscoelastic half-space is represented with different configurations of springs (elasticity) and dashpots (viscosity). A spring in series with a dashpot represents a simple univiscous Maxwell rheology (Figure 2.8a), which reproduces a non-recoverable creep. In contrast, a spring in parallel with a dashpot (Figure 2.8b) represents a recoverable Kelvin rheology.

The simplest model that describes both Kelvin and Maxwell materials is a Standard 
a) Maxwell

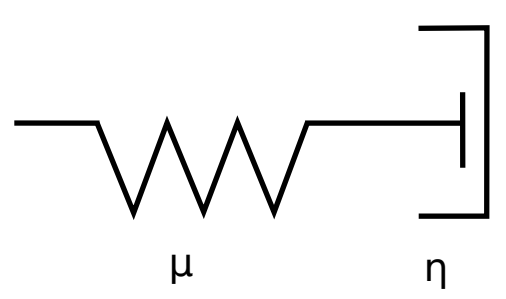

b) Kelvin

$\mu$

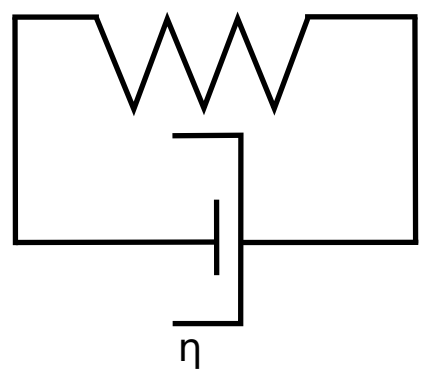

Figure 2.8: Schematic illustration of different rheology models: a) Maxwell rheology: a spring in series with a dashpot b) Kelvin rheology: a spring in parallel with a dashpot.

Linear Solid (SLS) model (Table 2.1; Wang et al., 2006; Hetland and Hager, 2005). A Maxwell element in series with a Kelvin element makes a bi-viscous Burger's body with two phases of viscoelastic creep (Table 2.1; Hetland and Hager, 2005; Pollitz, 2003b). Either by increasing the number of vertical layers or using a Burger's body, postseismic mechanisms with two mechanical time scales or more can be reproduced. However, heterogeneous rheology in which a weaker material is embedded within a stronger medium can also cause multiphases in postseismic signals (Hetland and Hager, 2005).

We summarise mechanical analogue models in terms of springs and dashpots and corresponding stress-strain constitutive laws using equations presented by Hetland and Hager (2005) and Segall (2010) in Table 2.1, for Maxwell and SLS materials, and for a Burger's body. For instance, the constitutive law for a Burger's body is written as

$$
\sigma+a_{1} \dot{\sigma}+a_{2} \ddot{\sigma}=a_{3} \epsilon+a_{4} \dot{\epsilon}
$$

The material behaviour of a Burger's body depends on the stress, the first and second derivatives of the stress, strain, and the first derivative of the strain. The coefficients $a_{1}, a_{2}, a_{3}$ and $a_{4}$ are functions of shear modulus and viscosity.

We adopt a univiscous Maxwell rheology and a Burger's body in Chapter 3 and 4, to analyse postseismic displacement following the Dusky Sound earthquake.

\section{Analytical viscoelastic solutions}

The Correspondence Principle simplifies solutions for a viscoelastic model, represented with an elastic layer overlying a viscoelastic half-space, to a two-layer elastic model. This elastic model consists of upper and lower layers with different elastic properties. Replacing the elastic moduli with the Laplace transformed moduli introduces a Lapla- 
Table 2.1: Mechanical analogue model and material behaviour for viscoelastic materials

\begin{tabular}{|c|c|c|}
\hline & $\begin{array}{c}\text { Mechanical analogue } \\
\text { model }\end{array}$ & $\begin{array}{c}\text { Material } \\
\text { behaviour }\end{array}$ \\
\hline Maxwell & & $\dot{\epsilon}=\left(\frac{\dot{\sigma}}{2 \mu}\right)+\left(\frac{\sigma}{2 \eta}\right)$ \\
Linear \\
Solid
\end{tabular}

cian variable $s$ in the corresponding viscoelastic solutions (Equation 2.22). Taking the inverse Laplace transform, converts the Laplacian variable $s$ to a time variable $t$. Therefore, in contrast with instantaneous elastic solutions, viscoelastic solutions are time-dependent.

For a model consisting of elastic upper and lower layers with different elastic properties Chou (1966) and Rybicki (1971) derived Equation 2.19, for the one-dimensional displacement field $d(x)$ due to strike-slip motion on a fault.

$$
d(x)=\left(\frac{\Delta u}{\pi}\right)\left( \pm\left(\frac{\pi}{2}\right)-\tan ^{-1}\left(\frac{x}{D}\right)+\sum_{n=1}^{\infty}\left(\frac{\mu_{c}-\mu}{\mu_{c}+\mu}\right)^{n} \tan ^{-1}\left(\frac{2 D x}{4 n^{2} H^{2}-D^{2}+x^{2}}\right)\right)
$$

where $\Delta u$ is slip on the fault with a maximum depth $D$, embedded in the upper elastic layer with thickness $H$, and \pm represents the sign of the position $x$. $\mu_{c}$ and $\mu$ are the shear moduli of the layers. For a material with a linear viscosity, the Laplace transform of Equation 2.19 is expressed as Equation 2.21. For different rheological assumptions, $\hat{\gamma}(s)$ (Equation 2.20) is first calculated and then the inverse Laplace transform of Equation 2.21 is taken. The analytical solutions obtained with this method are calculated for specific cases of a strike-slip fault within an elastic layer overlying a 
Table 2.2: Stress and strain coefficients in equations 2.13 and 2.14, specific forms of $\hat{\gamma}^{n}(s)$ and the corresponding inversely transformed forms of $\gamma^{n}(t)$ for Maxwell and SLS materials.

\begin{tabular}{|c||c||c|}
\hline & Maxwell & SLS \\
\hline Laplace coefficients & $\phi_{0}=1 ; \psi_{0}=0$ & $\phi_{0}=\frac{\mu_{v}+\mu_{e}}{\mu_{v}} ; \psi_{0}=\mu_{e}$ \\
& $\phi_{1}=\left(\frac{\eta_{m}}{\mu_{m}}\right)=\tau_{M} ; \psi_{1}=\eta_{m}$ & $\phi_{1}=\left(\frac{\eta_{v}}{\mu_{v}}\right)^{n}=\tau_{K} ; \psi_{1}=\mu_{e} \tau_{K}$ \\
\hline Specific form of $\hat{\gamma}^{n}(s)$ & $\hat{\gamma}^{n}(s)=\left(\frac{1}{2 \tau_{m}}\right)^{n}\left(\frac{1}{s+1 / 2 \tau_{m}}\right)^{n}$ & $\hat{\gamma}^{n}(s)=\left(\frac{\mu}{2 \mu_{v} \tau_{K}}\right)^{n}\left(\frac{1}{s+\frac{2 \mu_{v}+\mu}{\mu_{v} \tau_{K}}}\right)^{n}$ \\
\hline Specific form of $\gamma^{n}(t)$ & $\gamma^{n}(t)=\left(\frac{1}{2 \tau_{m}}\right)^{n} \frac{1}{(n-1) !} t^{n-1} \exp \left(-t \frac{1}{2 \tau_{m}}\right)$ & $\gamma^{n}(t)=\left(\frac{1}{2 \tau_{K}}\right)^{n} \frac{1}{(n-1) !} t^{n-1} \exp \left(-t \frac{3}{2 \tau_{K}}\right)$ \\
\hline
\end{tabular}

linear viscoelastic half-space (Equation 2.22).

$$
\begin{gathered}
\hat{\gamma}(s)=\left(\frac{\hat{\mu}_{c}-\hat{\mu}}{\hat{\mu}_{c}+\hat{\mu}}\right) \\
\hat{d}(x, s)=\left(\frac{\Delta \hat{u}(s)}{\pi}\right)\left(\left(\frac{\pi}{2}\right)-\tan ^{-1}\left(\frac{x}{D}\right)\right)+ \\
\left(\frac{1}{\pi}\right)\left(\sum_{n=1}^{\infty} \Delta \hat{u}(s) \hat{\gamma}^{n}(s) \tan ^{-1}\left(\frac{2 D x}{4 n^{2} H^{2}-D^{2}+x^{2}}\right)\right)
\end{gathered}
$$

Finally, taking the inverse Laplace transform of Equation 2.21 leads to

$$
d(x)=\left(\frac{\Delta u}{\pi}\right)\left(\frac{1}{2}-\tan ^{-1}\left(\frac{x}{D}\right)\right)+\left(\frac{1}{\pi}\right) \sum_{n=1}^{\infty} \Delta u * \gamma^{n}(t) \tan ^{-1}\left(\frac{2 D x}{4 n^{2} H^{2}-D^{2}+x^{2}}\right)
$$

where $*$ represents convolution in time.

We summarise the stress and strain coefficients in equations 2.13 and 2.14, specific forms of $\hat{\gamma}^{n}(s)$ and the corresponding inversely transformed forms of $\gamma^{n}(t)$ for Maxwell and SLS materials in Table 2.2. The material behaviour of a Burger's body follows Equation 2.18 but due to the complexity of the equations and their derivations, we do not include the corresponding $\hat{\gamma}^{n}(s)$ and $\gamma^{n}(t)$ in that case. For the complete derivations of Equation 2.20 and its inverse form see Hetland and Hager (2005).

\section{More realistic viscoelastic models}

We have previously discussed the simplest viscoelastic solution for a case of a strikeslip fault embedded in an elastic layer overlying a viscoelastic half-space. However, developing analytical forms of viscoelastic solutions is not generally possible unless the effect of heterogeneity within the media, gravity or other complexities associated with more realistic viscoelastic models can be discounted. Accordingly, in order to invert complex forms of viscoelasic solutions in the frequency domain into the time domain, time-consuming numerical methods such as the Haskell propagator algorithm (Haskell, 


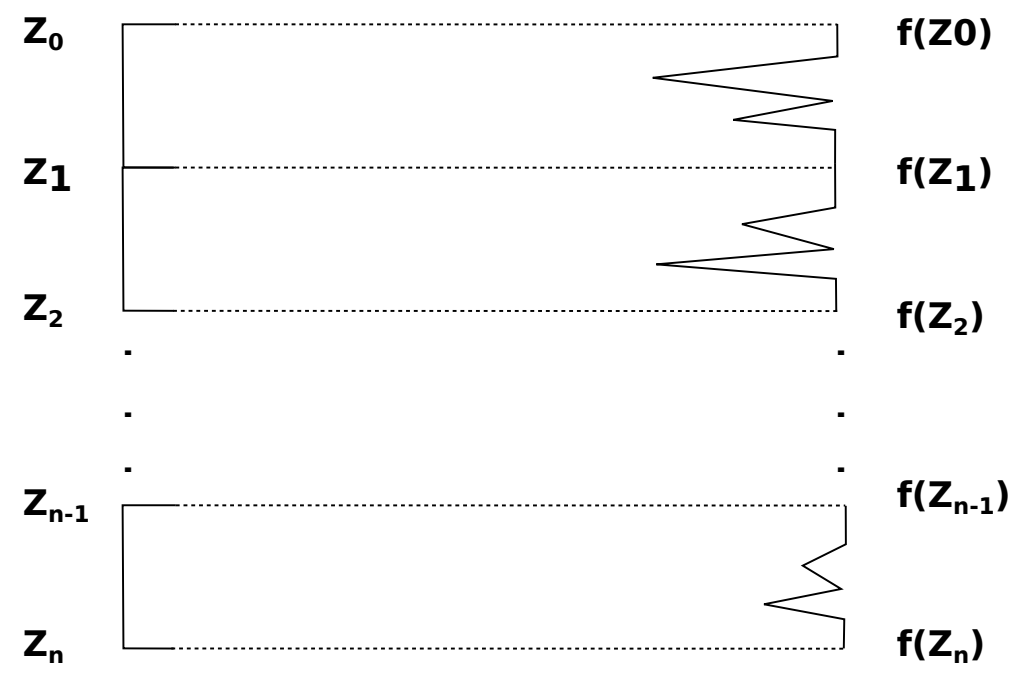

Figure 2.9: Schematic illustration of a propagator matrix being used to transform information throughout horizontally stratified layers.

1953) and the Hankel transform have been developed.

Semi-analytical algorithms are applied to reduce the computational time, using the collocation technique or full normal mode analysis and were the earliest tools for solving the Laplace transform inversion of viscoelastic solutions (Schapery, 1967; Peltier, 1985). Although the collocation technique is less time-consuming than full normal mode analysis, it requires preliminary information about the relaxation times (see Mitrovica and Peltier, 1992).

Several studies have attempted to derive semi-analytical solutions using the propagator matrix method for the layered viscoelastic representations. The gravitational layered earth models of Rundle (1980), Iwasaki (1985) and Fukahata and Matsu'ura (2006) and spherical geometry models without (Pollitz, 1992) and with considering the effects of gravity by Pollitz (1997) are examples of such semi-analytical solutions.

For layered elastic and viscoelastic models, the propagator matrix method attempts to simplify the boundary-value-problem to linear matrix equations (Gilbert, 1983). If $f(z)$ is a vector of $N$ variables, the transformation from the layer $z_{0}$ to $z_{n}$ is written in the form of propagator matrices $M$ as follows (Figure 2.9):

$$
f\left(z_{n}\right)=M\left(z_{n}, z_{n-1}\right) \cdots M\left(z_{2}, z_{1}\right) M\left(z_{1}, z_{0}\right) f\left(z_{0}\right)
$$

The roles of propagator matrices for analysing elastic or viscoelastic responses to dislocation sources in stratified earth models are: 1) To take stress-displacement vectors and propagate them to top or bottom layers; and 2) To assure that traction-free boundary conditions are met (Gilbert, 1983). 
For instance, the homogeneous governing equation derived from the equilibrium equation for the stress-displacement vector $v=\left[d_{3}, \sigma_{23}\right]$ is expressed in the general form

$$
\frac{d v(z)}{d z}=A v(z)
$$

where $z$ is the vertical component. Matrix $A$ consists of $4 \times 4$ elements as functions of the elastic constants $\lambda$ and $\mu$ and also a wavenumber $k$. The wavenumber is introduced as the result of applying the Fourier transform to the governing equation in order to reduce the partial differential equation to an ordinary differential equation.

$$
A=\left[\begin{array}{cccc}
0 & k & 1 / \mu & 0 \\
\frac{-k \lambda}{\lambda+2 \mu} & 0 & 0 & \frac{1}{\lambda+2 \mu} \\
\frac{4 \mu k^{2}(\lambda+\mu)}{\lambda+2 \mu} & 0 & 0 & \frac{k \lambda}{\lambda+2 \mu} \\
0 & 0 & -k & 0
\end{array}\right]
$$

The propagator matrix $M\left(z, z_{0}\right)$ for propagating from $z_{0}$ to $z$ in which $v(z)=M\left(z, z_{0}\right) v\left(z_{0}\right)$ is given by

$$
M\left(z, z_{0}\right)=I+\int_{z_{0}}^{z} A\left(\xi_{1}\right) d \xi_{1}+\int_{z_{0}}^{z} A\left(\xi_{1}\right)\left[\int_{z_{0}}^{\xi_{1}} A\left(\xi_{2}\right) d \xi_{2}\right] d \xi_{1}+\cdots,
$$

Similarly, the inhomogeneous form of an equilibrium equation equivalent to Equation 2.24 is written as

$$
\frac{d v(z)}{d z}=A v(z)+h(z)
$$

where $h$ represents the source term. Using the propagator matrix $M\left(z, z_{0}\right)$, the general solution of the inhomogeneous equilibrium equation is expressed in the form

$$
v(z)=M\left(z, z_{0}\right) v\left(z_{0}\right)+\int_{z_{0}}^{z} M(z, \xi) h(\xi) d \xi
$$

(e.g. Aki and Richards, 1980). We do not intend to discuss the equilibrium equations here and their derivations and thus all equations are presented in their general forms. Further details can be found in Segall (2010).

Singh (1970) and Sato (1971) proposed two types of propagator matrices, namely upgoing and down-going, respectively. The up-going and down-going propagator matrices are numerically stable below and above the source, respectively. Therefore, the downgoing propagator matrix produces stable solutions for the surface displacement and stress field. We must consider that unless unified up-going and down-going propagator matrices are used, the internal displacement is numerically unstable (Fukahata and Matsu'ura, 2005). To address this, Fukahata and Matsu'ura (2005) derived general expressions for the internal displacement field for a multi-layered elastic half-space and Fukahata and Matsu'ura (2006) for the viscoelastic half-space, using the Correspondence Principle. In Chapter 4, we apply the semi-analytical method of Fukahata 


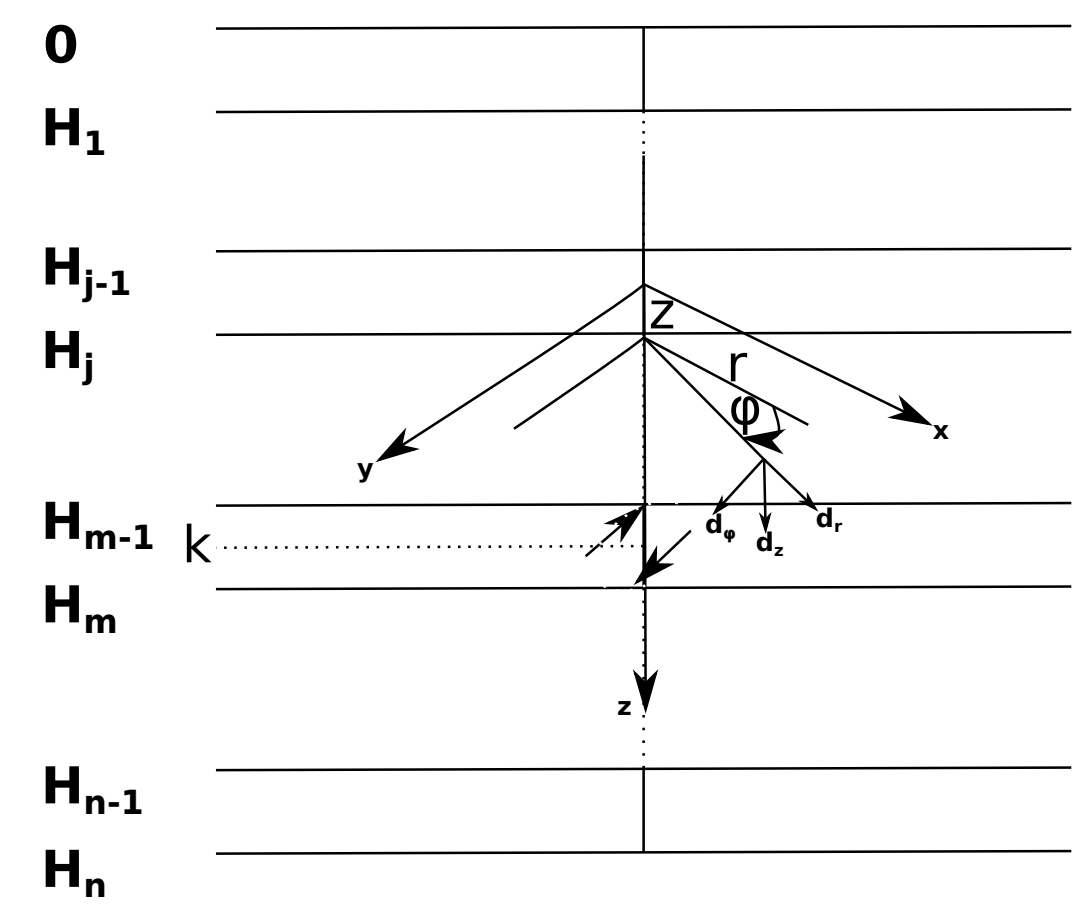

Figure 2.10: Dislocation source at a depth $d$ within a multi-layered viscoelastic half-space and viscoelastic slip response at point $(r, \phi, z)$ (Fukahata and Matsu'ura, 2006)

and Matsu'ura (2006) in order to calculate viscoelastic Green's functions (viscoelastic responses to unit slip). The viscoelastic slip-response function estimated by Fukahata and Matsu'ura (2006) consists of instantaneous elastic and viscoelastic transient terms. The elastic term is expressed by the Heaviside step function $H(t)$ and the transient term by the superposition of different decay time functions (Equation 2.31). We only present equations for the $\mathrm{z}$ component of displacement $d_{z}$ in a cylindrical coordinate system $(r, \phi, z)$ in a medium with elastic constants $\mu_{l}$ and $\gamma_{l}$ (Figure 2.10). The arbitrary point and the dislocation source are located in layer $j$ and $k$, respectively.

For an elastic multi-layered half-space the vertical component of elastic displacement $\left(d_{z}^{e}\right)$ is written as

$$
d_{z}^{e}\left(r, \phi, z, t, \mu_{l}, \gamma_{l}\right)=H(t) \frac{\Delta u}{4 \pi} \int_{0}^{\infty} \xi Y_{2}^{e}\left(z, \xi, j, \mu_{l}, \lambda_{l}\right) J(r, \phi, \xi) d \xi
$$

where $J$ is calculated from Bessel functions (see Watson, 1995) of order 0-2 (details in Fukahata and Matsu'ura 2006). More details are given for calculating $Y_{k}^{e}$ by Fukahata and Matsu'ura (2006). The elastic components are denoted by superscript $e$. For the viscoelastic multi-layered half-space using the Correspondence Principle, the Laplace transform of the $\mathrm{z}$ component of displacement is expressed as

$$
\widetilde{d}_{z}(r, \phi, z, s)=\frac{\Delta u}{4 \pi} \int_{0}^{\infty} \xi \widetilde{Y}_{2}(z, s, j) J(r, \phi) d \xi
$$


After operating the inverse Laplace transform and obtaining $Y_{k}, \widetilde{Y}_{k}$ is replaced by Equation 2.31 in Equation 2.30 and the viscoelastic displacement components are resolved.

$$
Y_{k}(z, t ; j)=H(t)\left[Y_{k}^{e}\left(z, j, \mu_{l}, \lambda_{l}\right)-\sum_{i=1}^{M} a_{i}\left[1-\exp \left(b_{i} t\right)\right]\right]
$$

The transient creep is expressed by superposition of $M$ exponential decaying functions with $M$ values of coefficients $a_{i}$ and $b_{i}$ (see Fukahata and Matsu'ura 2006 for more details of calculating the coefficients).

In addition to vertical heterogeneity of viscoelastic properties, the mechanical behaviour of the ductile material within the lower crust and upper mantle vary with lateral variations of viscosity, temperature and elastic thickness. Pollitz (2003a) considered the relaxation of the heterogeneous spherical viscoelastic model and introduced semi-analytical solutions which also account for the coupling of viscoelastic rebound and gravity.

Although the semi-analytical inversion methods were first proposed to reduce computational time, purely numerical methods are no longer impossible tasks. Wang et al. (2006) presented a numerical method using the Riemann-Mellin contour integral and the Fast Fourier Transform (FFT) technique to compute Green's functions in a viscoelasticgravitational half-space. The use of FEM (e.g. Aagaard et al., 2009, 2013, 2015) to compute the viscoelastic solutions taking into consideration vertical and lateral heterogeneity, coupling with gravity and more complex geometries (e.g. slab geometry for subduction earthquakes) is another example of a fully-numerical viscoelastic methods. In Chapter 4, we consider lateral heterogeneity and the geometry of a slab in a simulated subduction earthquake in our simulations using PyLith (Aagaard et al., 2009, 2013, 2015).

\section{Coupling with gravity}

Fault movements perturb gravitational equilibrium (Segall, 2010). Over short temporal and small spatial scales, the perturbation of gravitational forces and potential is negligible. However, for long-term viscoelastic effects, especially after large earthquakes, the effect of the gravity field must be considered as it may change the static displacement by up to 10\% (e.g. Fernández et al., 1996, 1999).

Rundle (1982) solved for static displacement in a self-gravitating earth model with gravity $g$ as a constant body force and density $\rho$. In this case, Equation 2.25 therefore 
becomes

$$
A=\left[\begin{array}{cccc}
0 & k & 1 / \mu & 0 \\
\frac{-k \lambda}{\lambda+2 \mu} & 0 & 0 & \frac{1}{\lambda+2 \mu} \\
\frac{4 \mu k^{2}(\lambda+\mu)}{\lambda+2 \mu} & k \rho g & 0 & \frac{k \lambda}{\lambda+2 \mu} \\
k \rho g & 0 & -k & 0
\end{array}\right]
$$

The eigenvalues of Equation 2.25 without considering the effects of gravity, are $\pm|k|$. With gravity we have eigenvalues $\omega= \pm|k|\left[1 \pm \frac{k_{g}}{k}\right]^{1 / 2}$ for matrix $A$ (Equation 2.32) where the gravitational wavenumber is

$$
k_{g}=\frac{\rho g}{\sqrt{\mu(\lambda+2 \mu)}}
$$

If $k \leq k_{g}$, the eigenvalues are imaginary and the boundary value problem has no solution. At long time scales, when the effective shear modulus is small, the gravitational wavenumber becomes large enough to make the boundary value problem unstable (Wang, 2005; Wang et al., 2006). Wang (2005) corrected this issue by using the Adams-Williamson condition in the governing equations

$$
\frac{d \rho}{d z}=\frac{\rho^{2} g}{K}
$$

where $K=\lambda+2 \mu / 3$ is the bulk modulus. The modified governing equations lead to the modified gravitational wavenumber and modified eigenvalues (equations 2.35 and 2.36, respectively):

$$
\begin{gathered}
k_{g}=\frac{\rho g}{\sqrt{\mu(\lambda+2 \mu})} \Longrightarrow \tilde{k_{g}}=\frac{\rho g}{\sqrt{4 / 3 K(\lambda+2 \mu)}} \\
\omega= \pm|k| \sqrt{1 \pm \frac{k_{g}}{k}} \Longrightarrow \omega= \pm \sqrt{k^{2}+2 / 3\left({\tilde{k_{g}}}^{2} \pm \tilde{k_{g}} \sqrt{{\tilde{k_{g}}}^{2}-k^{2}}\right)}
\end{gathered}
$$

Additionally, using numerical methods such as the FEM, the singularity due to gravity can also be artificially resolved by assuming a finite thickness of the half-space and imposing constraints on the bottom interface of the layer (see Wu, 1992; Zhong et al., 2003; Wu, 2004).

\section{Multiphase postseismic creep and layering}

Several researchers have shown that the postseismic signal can reveal single or multiple phases of relaxations. Savage et al. (2003) fit exponential functions to the postseismic relaxation after the $1992 M_{w} 7.3$ Landers and $1999 M_{w} 7.1$ Hector Mine earthquakes, California, of the form $u_{\exp }=A\left[1-\exp \left(-\frac{t}{\tau_{\exp }}\right)\right]$, representing a single process with a 
relaxation time of $\tau_{\text {exp }}$. Herring (2003) analysed the postseismic signal measured by the Southern California Integrated GPS network after the 1994 Northridge earthquake and described the observed displacement with logarithmic functions of the form $u_{\ln }=B[1+$ $\ln \left(-\frac{t}{\tau_{e l n}}\right)$ ]. Savage et al. (2005) explained postseismic displacements in the epicentral area, after the $1992 M_{w} 7.3$ Landers, $1999 M_{w} 7.6$ Chi-Chi (central Taiwan), $1999 M_{w}$ 7.1 Hector Mine, $2002 M_{w} 7.9$ Denali (Alaska), $2003 M_{w} 6.5$ San Simeon and $2004 M_{w}$ 6.0 Parkfield earthquakes (California), in terms of logarithmic functions.

Postseismic relaxation with two phases can be well-described by logarithmic functions. Hetland and Hager (2005) examined how layering and different rheologies affect viscoelastic relaxation. In addition to the viscoelastic half-space, they considered a viscoelastic middle layer between the upper elastic layer and the lower half-space in three-layer models. In doing so, Hetland and Hager (2005) showed that a model consisting of an elastic layer overlying a Burger's half-space (elastic-Burger's) imposes two mechanical time scales with logarithmic relaxation. The weaker the Kelvin element in the Burger's body, the larger the difference between the resulting time scales are.

The elastic-Maxwell-Maxwell model causes large-wavelength displacement if the relaxation time associated with the middle layer is long. Both exponential and logarithmic functions can describe the temporal displacement related to the elastic-MaxwellMaxwell model.

A three-layer model with a Maxwell rheology in the middle layer and a Burger's body in the half-space behaves differently near the fault if the Maxwell element is relatively weak with respect to the half-space. The greater the distance from the fault, the greater the effect the Burger's body has. If the Maxwell middle layer is strong, two phases of relaxation at any distance from the fault are produced and the model approaches the solutions of the elastic-Burger's model. Generally, this three-layer model exhibits up to three time-scales in the postseismic relaxation. Interestingly, swapping the rheologies of the middle layer and the half-space results in four mechanical time scales from the coupling of the two upper layers and the two lower layers. The number of the mechanical time scales increases to up to five by associating a Burger's body to the middle layer and a Burger's Body to the lower half-space. In Chapter 3, we only examine the effects of a bi-viscous Burger's half-space on the multiphase postseismic displacement following the Dusky Sound earthquake. 


\section{Literature review of viscoelastic models used in previous studies}

Having reviewed different viscoelastic models with a broad range of rheologies and corresponding viscosities, it is beneficial to compare the different assumptions upon which models of postseismic signals have been based. A summary of some of the parameters used in these studies is presented in Figure 2.11. For more details, we classify the information extracted from 88 studies in Tables B.1, B.2 and B.3 (Appendix B). From this literature review, key considerations are highlighted:

Non-uniqueness of postseismic solutions: Rousset et al. (2012a) demonstrated the non-uniqueness of postseismic solutions for the 1999 Chi-Chi earthquake based on a sensitivity test. It was shown that surface postseismic displacement is not sensitive to the rheology of the upper mantle. As Rousset et al. (2012a) explained, understanding the subsurface structure of the earth using geodesy can often present non-unique solutions. Different studies infer different mechanisms of postseismic displacements. For instance, the postseismic displacement observed in the period 2007-2011 after the Kuril earthquake doublet (Russia), can be explained either by a Maxwell rheology with varying viscosity from $2 \times 10^{17}$ to $10^{18} \mathrm{~Pa} \cdot \mathrm{s}$ within four years or by a power law creep (Kogan et al., 2013). In a similar way, Rollins et al. (2015) considered several models to explain the postseismic displacement following the the $2010 \mathrm{M}_{\mathrm{W}} 7.2$ El Mayor-Cucapah earthquake including: 1) Afterslip within the lower crust and assuming a zone with a low viscosity and Newtonian asthenosphere; 2) Assuming a zone with a low viscosity and mantle with a power-law rheology and Newtonian asthenosphere.

Improving the uniqueness of postseismic solutions: Using other observations from sources such as tomography, thermo-kinematics models, seismicity and the local hydro-isostatic adjustment associated with the mantle flow can improve the uniqueness of geodetic problems. Hoechner et al. (2011) demonstrated that the viscoelastic effect of a Maxwell rheology combined with an afterslip model underestimates the postseismic geoid changes observed by the GRACE mission following the $\mathrm{M}_{\mathrm{W}} 9.12004$ Sumatra earthquake. However, a Burger's body with a transient viscosity of $10^{18}$ $\mathrm{Pa} \cdot \mathrm{s}$ and steady-state viscosity of $10^{19} \mathrm{~Pa} \cdot \mathrm{s}$ within the viscoelastic layer underlain by the elastic layer, $40 \mathrm{~km}$ thick, agrees with the geoid changes. Hoechner et al. (2011) suggested that installing ocean bottom pressure gauges, measuring geoid changes by the GRACE mission, in addition to space-based geodetic observations are useful tools to distinguish between bi-viscous and afterslip as the main postseismic mechanisms. Furthermore, Panet et al. (2010) suggested that, as GPS data are less sensitive to deeper processes, gravity signals observed by the GRACE mission when used as complementary data overcome the small-scale and sparsity of GPS measurements. They 
showed how the estimated mantle and lower-mantle viscosity can be improved using large-scale gravity signals. The other advantage of constraining viscoelastic relaxation models with gravity observations is the sensitivity of gravity to the vertical component of displacement (Panet et al., 2010; Hoechner et al., 2011).

Slab geometry of subduction earthquakes Assuming a realistic slab geometry or a simple elastic over viscoelastic plane earth model leads to different postseismic displacements. Masterlark (2003), Hu et al. (2004) and Pollitz et al. (2008) explained how the damping effect of the slab can be mitigated by either reducing the viscosity of the mantle on the continental side, or the thickness of the elastic layer.

Lateral heterogeneity of viscoelastic properties Yamasaki et al. (2014) considered a localised weak zone with an exponentially decreasing viscosity at depth, to explain GPS observations within a six month period following the 1999 İzmit earthqauke (North Anatolian Fault Zone). Le Pourhiet et al. (2014) and Yamasaki et al. (2014) associated the presence of low-viscosity zones below the elastic layer with either shear heating, enhanced fluid content, fabric development or grain size reduction. They suggested that the lateral variations of rheology sometimes explain the geodetic observations more efficiently than the stratified layers.

\subsubsection{Transient creep}

Transient creep causes logarithmic decay of ground displacement following large earthquakes. Benioff (1951) interpreted postseismic relaxation in terms of a mechanism similar to the time-varying creep recovery observed in low-temperature laboratory experiments. Perfettini and Avouac (2004) inferred logarithmic creep decay from geodetic observations at a rate similar to the decay of the cumulative number of aftershocks following the Chi-Chi earthquake. Additionally, Savage et al. (2005) showed that GPS observations following multiple large earthquakes reveal logarithmic decay with time in the epicentral area during the early postseismic stages, which is primarily explained by two rheologies including frictional afterslip and a bi-viscous Burger's body.

Perfettini and Avouac (2004) considered rate-and-state frictional law in order to link the logarithmic decay of aftershocks to geodetic observations. Several authors postulate that the transient creep process is a result of stress-driven afterslip combined with viscoelastic flow (e.g. Johnson et al., 2009; Wang et al., 2009; Panet et al., 2010; Yamagiwa et al., 2015; Rollins et al., 2015). If so, the rapid logarithmic decay at the early stage of postseismic time period can be explained by afterslip being driven by elastic and viscoelastic stress changes induced by earthquakes. Johnson et al. (2009) devel- 


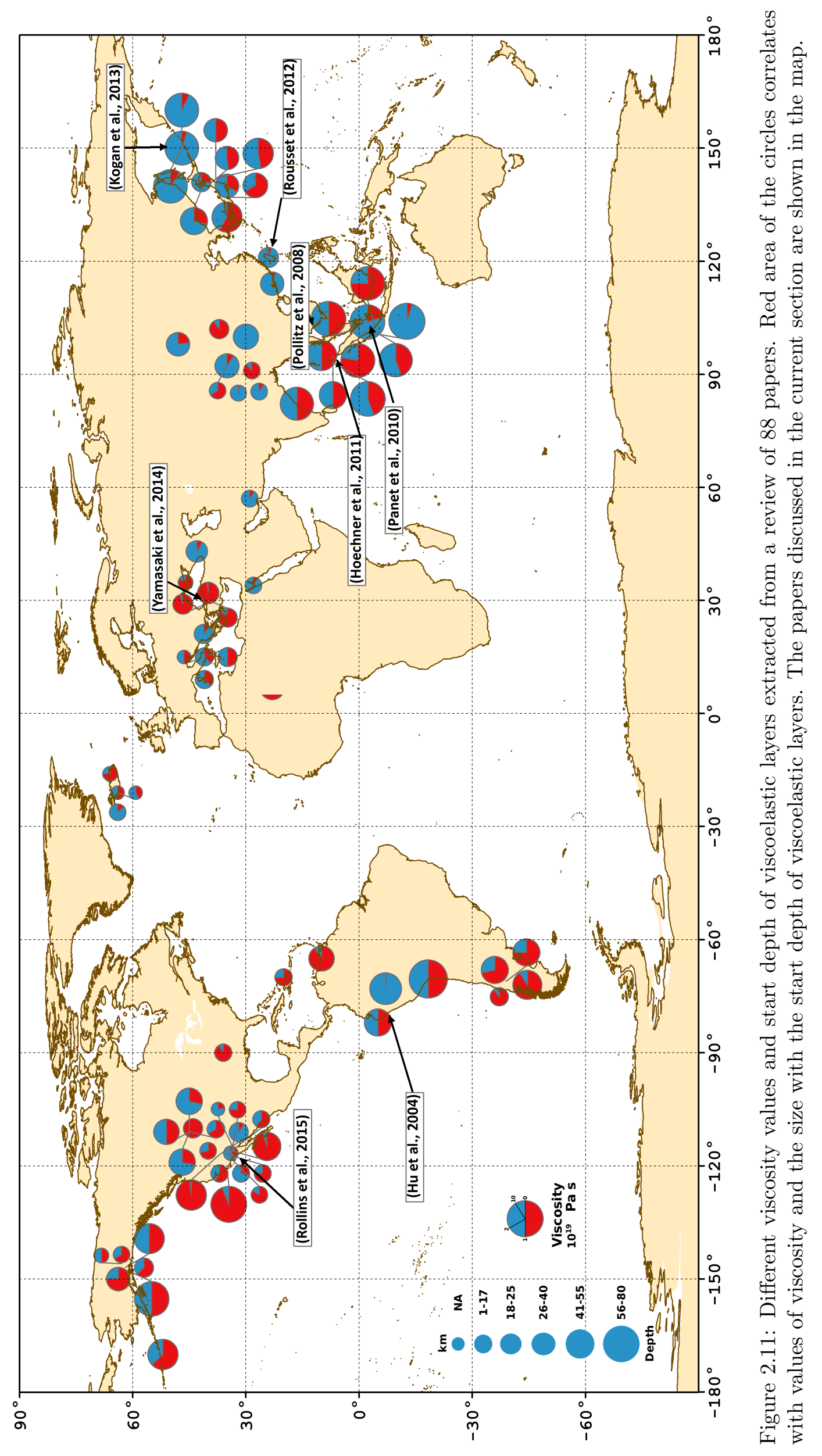


oped a numerical coupled afterslip and viscoelastic relaxation model in order to model postseismic displacement following the Denali, Alaska, earthquake. They assumed that afterslip obeys the rate-strengthening friction law and solved for the numerical integral in Equation 2.37 in order to estimate slip $(s)$ at time $t$

$$
\tau_{i}(t)=\tau_{i}^{c}(t)+\int_{0}^{t} G_{i}\left(t-t^{\prime}\right) v\left(t^{\prime}\right) d t^{\prime}
$$

where $\tau_{i}^{c}(t)$ is the shear stress induced by coseismic elastic and viscoelastic responses on each patch of the fault (patch $i$ ). $G_{i}$ is the time-dependent viscoelastic Green's functions (Fukahata and Matsu'ura, 2006), and $v$ is the time-dependent slip-rate.

Barbot and Fialko (2010a) presented a framework in which a unified representaion of postseismic mechanisms is considered by defining viscoelastoplastic rheology and different relaxed quantities. These quantities are deviatoric stress for viscoelastic relaxation, shear stress for creep and the trace of stress tensor for poroelastic rebound.

In addition to frictional afterslip, the Burger's rheology for the upper mantle with two transient and steady state viscosities has been shown to account for transient creep following several large earthquakes. Transient rapid decays observed in central Alaska following the Denali earthquake and beneath the Mojave Desert following the Hector Mine earthquake were the first such examples of using the Burger's rheology (Pollitz, 2005, 2003b). The bi-viscous rheology has since been used in several other studies to model the early rapid decay in the postseismic period (e.g. Árnadóttir et al., 2005; Pollitz et al., 2006b; Johnson et al., 2007; Pollitz et al., 2008; Hearn et al., 2009; Panet et al., 2010; Hoechner et al., 2011; Sun and Wang, 2015).

Short-term rapid decay of postseismic displacement is often explained in terms of very low values of transient viscosity $\left(<10^{17} \mathrm{~Pa} \cdot \mathrm{s}\right)$ and short relaxation times (e.g. Palano et al., 2009). Very short relaxation times are also consistent with the characteristics timescales of afterslip (a few months to a few years; Pritchard and Simons, 2006; Wang et al., 2012). Therefore, in Chapters 3 and 4, we interpret the logarithmic transient displacement following the Dusky Sound earthquake in terms of afterslip and viscoelastic responses to both coseismic stress changes and afterslip mechanism.

\section{Fault frictional afterslip}

A rate-strengthening rheology is commonly assumed to be the dominant type of friction during the postseismic time period inducing afterslip (Lapusta et al., 2000; Rice et al., 2001; Barbot et al., 2009). This form is derived from the general rate-and-state- 
dependent friction law of Ruina (1983) and Dieterich (1994)

$$
\begin{aligned}
& f=f_{0}+a \ln \left(\frac{v}{v_{0}}\right)+b \ln \theta \\
& f \equiv f_{0}+a \sinh ^{-1}\left(\frac{v}{2 v_{0}} \theta^{\frac{b}{a}}\right)
\end{aligned}
$$

where $f_{0}$ is the coefficient of friction at a reference time and reference slip rate $v_{0} . \theta$ is a state variable, $v$ is slip rate and $a$ and $b$ are frictional parameters. If $\frac{b}{a}>1$, the workhardening parameter $b / a$ is rate-weakening, whereas if $\frac{b}{a}<1$, it is rate-strengthening.

Assuming, $\tau=f \sigma^{\prime}$, where $\tau$ is shear stress in the direction of sliding on a fault and $\sigma^{\prime}$ is the effective normal stress, the following extra assumptions have been made in order to derive the rate-strengthening form of the friction law (Lapusta et al., 2000; Rice et al., 2001; Barbot et al., 2009):

- Normal stress changes are negligible with respect to lithostatic and tectonic stress components;

- There are no weakening processes;

- Friction is steady-state; and

- Coulomb stress changes induced by earthquakes are the dominant stress changes inducing further afterslip.

Accordingly, the slip rate induced by coseismic stress changes is derived from Equation 2.38 in the form of Equation 2.39

$$
v=2 v_{0} \sinh ^{-1}\left[\frac{\Delta \tau}{(a-b) \sigma}\right]
$$

where $\Delta \tau$ is shear stress changes and $(a-b) \sigma$ is the parameter of the model associated with the background normal stress and the frictional parameter $(a-b$; e.g. Lapusta et al., 2000; Rice et al., 2001; Barbot et al., 2009).

Assuming the component of earthquake-induced traction changes (in the direction of sliding on the fault surface) drives afterslip, the forward relation between the slip rate and the traction changes is defined by Equation 2.39. Alternatively, afterslip can be estimated using conventional methods by inverting the observed surface displacement (e.g. Freed, 2007; Kositsky and Avouac, 2010; Evans and Meade, 2012). Using forward modelling methods, we need to know the coseismic slip distribution, the geometry of the afterslip surface, the sliding direction or rake angle and the appropriate frictional properties. In contrast with the forward method, inverting the surface displacement data does not consider the traction changes on the fault surface induced by the earthquake; Slip occurs either within or beyond the coseismic rupture patches. Moreover, in Equation 2.39, if $(a-b) \sigma \ll \Delta \tau$, the rate-strengthening afterslip model possesses a singularity. In addition, the general form of the rate-and-state dependent 
friction law requires knowledge of several properties such as state variable $\theta$ and frictional parameters $a$ and $b$.

In Chapter 5, we employ forward model of frictional afterslip from Equation 2.39 as a first-order approximation in order to understand the main postseismic mechanisms following the Canterbury earthquake sequence of 2010-2012. In Chapter 3 and 4, we discuss time-dependent inversion methods with which to estimate afterslip following the Dusky Sound earthquake in an elastic-viscoelastic medium.

\section{Time-dependent inversion of geodetic data}

Daily measurements of continuous GPS (cGPS) sites provide high-resolution crustal information with a horizontal accuracy of a few millimetres and poorer vertical accuracy (approximately three times larger, Heflin et al., 1992; King et al., 1995). Timedependent inversion of temporally dense observations measured from cGPS data enables estimation of transient slip associated with postseismic displacements. Therefore, providing a framework to invert temporal geodetic observations is of great use. Segall and Matthews (1997) developed the Network Inversion Filter (NIF) method to approximate slip using spatial basis functions $B_{k}(\xi)$ and temporal coefficients $c_{k}(t)$ of the form

$$
s(\xi, t)=\sum_{k=1}^{M} c_{k}(t) B_{k}(\xi)
$$

Slip through time is predicted in this method from the sum of steady-state slip and random walks. Segall and Matthews (1997) computed Kalman gain (see Anderson and Moore 2012) to estimate the update for each parameter and associated covariance matrix. The temporal smoothness of slip was controlled by a constant scaling parameter. Fukuda et al. (2004) subsequently modified the NIF method by applying a Monte Carlo Mixture Kalman Filter (MCMKF) in which the temporal smoothness varies as a stochastic variable. Consequently, if slip changes abruptly, MCMKF is capable of capturing the slip event. However, Fukuda et al. (2004) assumed spatially uniform fault slip in their method, a limitation later addressed by Fukuda et al. (2008) with combining a hierarchical Bayesian approach (MacKay, 1992) and state-space Kalman filtering (Kalman, 1960; Segall and Matthews, 1997; Kitagawa and Gersch, 2012). The effect of viscoelastic relaxation is not taken into account in these methods.

Estimating time-dependent slip distribution was also considered by Fukahata et al. (2004) using Akaike's Bayesian Information Criterion (ABIC) method (Akaike, 1977, 1980; Yabuki and Matsu'Ura, 1992; Ide et al., 1996). The advantage of this approach 

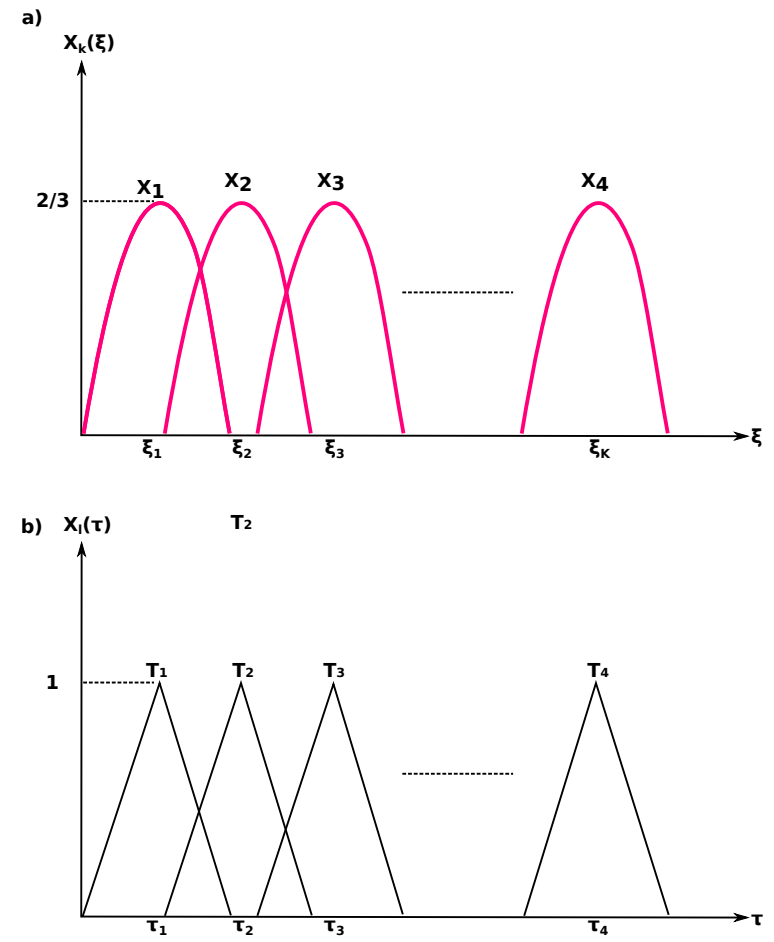

Figure 2.12: Schematic diagrams for a) Spatial basis b) Temporal basis functions used to model slip rates throughout ABIC method.

is that they could consider the effects of viscoelastic relaxation in the mantle. Slip rate is thus written as the superposition of temporal $T_{l}(t)$ and spatial basis functions $X_{k}(\xi)$ (Figure 2.12)

$$
v(\xi, t)=\sum_{k=1}^{K} \sum_{l=1}^{L} a_{k l} X_{k}(\xi) T_{l}(t)
$$

where $a_{k l}$ are coefficients determined from the inversion of geodetic observations using the ABIC method. The Bayesian framework used in the ABIC method incorporates the smoothness of the fault slip in time and space as a priori information by introducing two hyperparameters, $\alpha^{2}$ and $\beta^{2}$. Coefficients $a_{k l}$ and the hyperparameters are optimised in order to minimise the misfit with geodetic observations as well as the spatial and temporal roughnesses of fault slip.

In all the above methods, inversion of time-dependent geodetic observations is performed by the expansion of slip in terms of spatial and temporal basis functions. Kositsky and Avouac (2010) inverted geodetic time series with the inverse of elastic Green's functions multiplied by Singular Value Decomposition (SVD) of ground observations $D$. This method does not require use of a temporal hyperparameter. Temporal information is directly obtained from decomposition of temporally dense geodetic observations 
into spatial $(U)$ and temporal functions $(V)$, via

$$
\begin{gathered}
D_{r}=U_{r} \Lambda_{r} V_{r}^{\prime} \\
\mathbf{S}=G_{e}^{-1} U_{r}\left(\Lambda_{r} V_{r}^{\prime}\right)
\end{gathered}
$$

where $\mathbf{S}$ is time-dependent slip matrix and $G_{e}$ is the matrix of elastic Green's functions, $D_{r}$ is a filtered form of the full displacement matrix $D$ with $n$ observed components at $T$ epochs, of the dimensions $n \times T ; U_{r}$ is of dimension $n \times r$, representing the first $r$ columns of $U ; \Lambda_{r}$ is of dimension $r \times r$, formed from the first $r$ rows and columns of $\Lambda$, which contains singular values of $D$; and $V_{r}$ is of dimension $T \times r$ and represents the first $r$ columns of $V . r$ is the number of retained singular values and the optimal value of $r$ is selected by minimising the misfit between the displacement signal $D$ and the filtered signal $D_{r}$ (Kositsky and Avouac, 2010). If the single values are sorted from higher to lower values, we can perform filtering by single value truncation. Throughout this thesis, matrix transpose is denoted by prime $\left({ }^{\prime}\right)$ symbol.

Viscoelastic responses in the lower crust/upper mantle using the Principal Component Analysis-based Inversion Method (PCAIM) are not considered here. In Chapter 4, we propose an approach using semi-analytical (Fukahata and Matsu'ura, 2006) or hybrid numerical viscoelastic Green's functions (Aagaard et al., 2009, 2013, 2015) in order to invert the geodetic time series assuming an elastic-viscoelastic medium.

\subsubsection{Viscoelastic cycle models}

The ultimate aim of Chapter 4 of this thesis is to provide an inversion framework in which to constrain postseismic displacement mechanisms in an elastic-viscoelastic medium. This framework is useful to model long-term ground displacements measured from geodetic observations within a viscoelastic cycle. Studying a viscoelastic cycle encompassing repeated earthquakes, with corresponding postseismic mechanisms, and the longer-term interseismic period is a subject of ongoing research. The materials described in this section are not directly used in the following chapters, although different rheological models here compliment the assumptions in Chapters 3 and 4.

The concept of viscoelastic cycle models of periodic earthquakes is that the asthenosphere relaxes the stress imposed by the periodic earthquakes on the upper part of the fault, and aseismic creep on the lower segment. Postseismic relaxation within the asthenosphere thereafter transfers stress to the elastic layer. The idealised earthquake cycle with periodic earthquakes on the upper part of the fault has been assumed for viscoelastic cycle models in several studies under different rheological assumptions. 
Perfettini and Avouac (2004) proposed the Brittle Creeping Fault Zone (BCFZ) rheology for the region connecting the seismogenic zone (at temperatures $<250{ }^{\circ} \mathrm{C}$ ) to the region with temperatures $>400^{\circ} \mathrm{C}$ (Figure. 2.13a). The rheology for the BCFZ is assumed to be stable rate-strengthening, which does not produce earthquakes. During the interseismic period, the upper part of the fault is locked. The stress drop induced by the earthquake is transferred to the creeping part of the fault below the coseismic rupture zone, relaxing the stress produced by the upper part of the fault. Perfettini and Avouac (2004) assumed that microseismicity over the region below the locked fault is triggered by interseismic strain accumulation. However, the strain released by microseismicity is a fraction of the measured strain by the geodetic data (Perfettini and Avouac, 2004). Thus, rate-strengthening creep is suggested to be the primary mechanism explaining the geodetic observations.

An alternative mechanism is that, grain size reduction after repeated earthquakes localises the ductile shear zone displacement down-dip of the seismogenic zone (Montési and Hirth, 2003). Ductile transient creep is associated with power-law or Newtonian rheologies. For a ductile shear zone of thickness $h$, the slip rate depends on the power $n$ of the stress. For a Newtonian fluid with a linear rheology $(n=1)$ and a pre-exponential factor $A$

$$
v=A h \sigma^{n}
$$

Equilibrium requires that

$$
\frac{d \sigma}{d t}=K\left(v-v_{p}\right)
$$

A spring-slider system with tectonic- and earthquake-induced stresses is assumed, represented by a loading point velocity $v$ and a shear zone velocity $v_{p}$, respectively (Figure 2.14). $K$ is the stiffness of the spring and represents stress sources and shear zone geometry.

Assuming either rate-strengthening friction (Equation 2.39) or a power-law rheology (Equation 2.44) yields the differential equation (Equation 2.45) to be solved and thus an estimation of the fault slip history. Fault slip due to the rate-strengthening rheology evolves in time in the form of $\ln \left(1+\frac{t}{\tau_{R}}\right)$ with $\tau_{R}$ a material constant. For the power-law creep and a linear rheology, evolution of fault slip has the form $n\left(1-\left[1+\left(1-\frac{1}{n}\right) \frac{t}{\tau_{R}}\right]^{\frac{1}{1-n}}\right)$ and $\left(1-\exp \left(-\frac{t}{\tau_{R}}\right)\right)$, respectively (Montési, 2004).

Savage (2007) developed an analytical model of postseismic slip on the BCFZ with the rheology of Lomnitz solids. Based on the experiments of Lomnitz 1956, materials having the transient creep response are referred to as Lomnitz solids (Savage, 2007). In contrast with the model of Perfettini and Avouac (2004), the BCFZ extends to the 

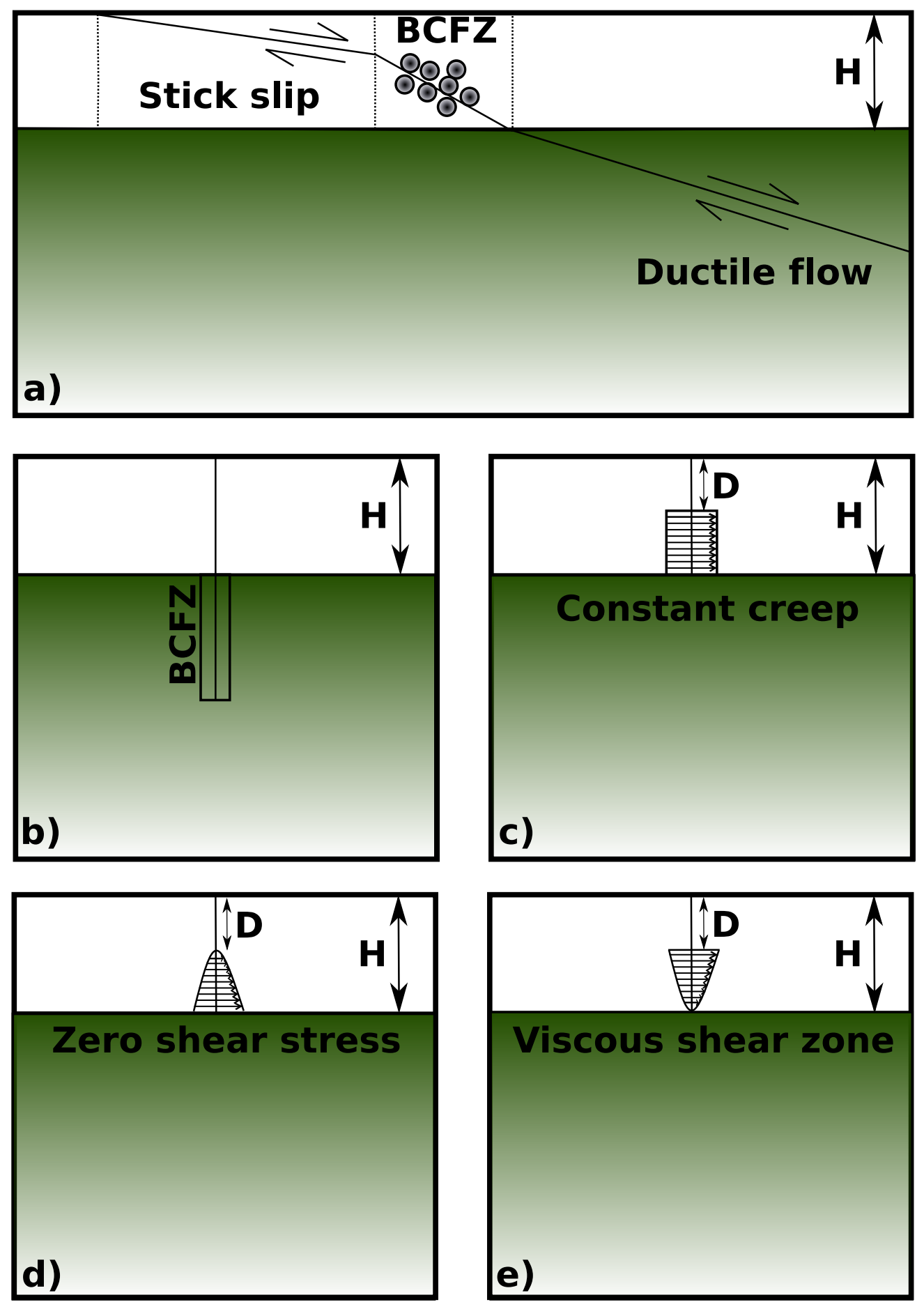

Figure 2.13: a) The BCFZ rheology and triggered microseismicity during the interseismic period, $\mathrm{BCFZ}$ is located in the region with temperatures between 250 and 400 below the coseismic rupture patch. b) BCFZ rheology extending to the viscoelastic subsurface. c) Viscoelastic cycle model with constant creep on the creeping part of the fault d) Viscoelastic cycle model over a zero shear stress zone. e) Viscoelastic cycle model over a viscous shear zone. 


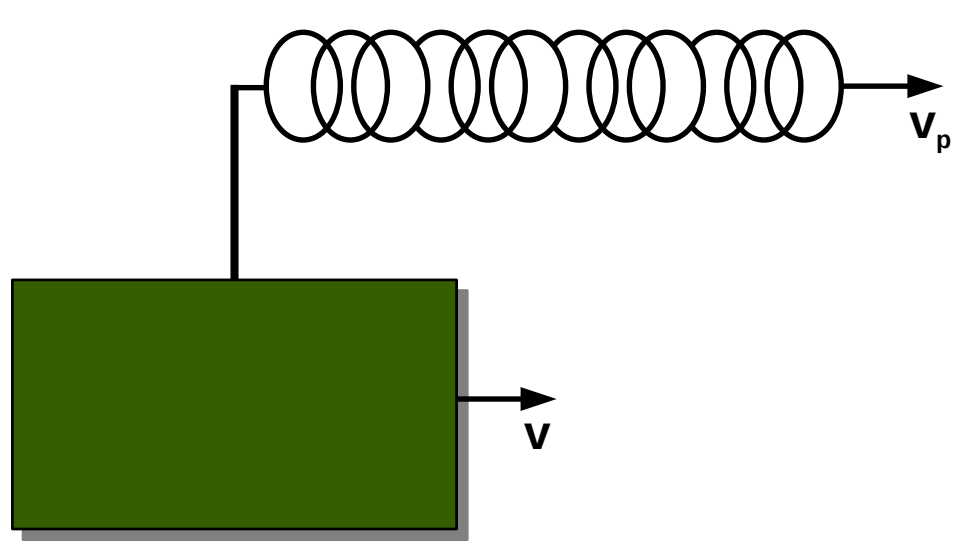

Figure 2.14: Spring-slider system

viscoelastic subsurface (Figure. 2.13b). The constitutive law for the Lomnitz solids is

$$
\sigma=\frac{\mu}{\left(1+q \ln \left(1+\frac{t}{\tau_{R}}\right)\right)} \epsilon
$$

where $\sigma$ is the imposed stress, $\epsilon$ is the strain response, $\mu, q$ and $\tau_{R}$ are material constants.

Figure. 2.13.(c-e) are illustrations for three different analytical cycle models that Johnson and Segall (2004) proposed for modelling creep along the San Andreas Fault System. All three viscoelastic cycle models consist of a vertical fault within an elastic layer, overlying a Maxwell viscoelastic half-space under the following assumptions: 1) constant creep on the lower part of the creeping fault (Figure 2.13c); 2) creep within a zero shear stress zone (Figure 2.13d); and 3) creep within a linear viscous shear zone (Figure 2.13f).

\subsubsection{Poroelastic rebound of a porous medium}

Understanding the role of fluids at different times and positions in an earthquake cycle is also important in order to interpret postseismic displacement. An instantaneous change of the confining stress due to an earthquake causes pore pressure changes and the formation of dilational and compressional zones (e.g. Peltzer et al., 1996, 1998). Later in the postseismic period, the fluid flows between these zones in order to equilibrate the pore pressure changes. This postseismic process is known as poroelastic rebound (e.g. Roeloffs, 1996; Peltzer et al., 1996, 1998). 
In this section, we discuss the following governing equations of poroelastic rebound

$$
\begin{array}{r}
(\lambda+2 \mu) \nabla(\nabla \cdot \vec{d})-\mu \nabla \times(\nabla \times \vec{d})-\alpha \nabla P_{p}=\vec{f} \\
Q^{-1} \frac{\partial P_{p}}{\partial t}-\chi \nabla^{2} P_{p}+\alpha \frac{\partial}{\partial t} \nabla \cdot \vec{d}=0
\end{array}
$$

where $\lambda$ and $\mu$ are the Lamé parameters, $d$ is displacement signal induced by poroelastic rebound, $P_{p}$ is pore pressure, $\chi$ and $Q^{-1}$ are Darcy conductivity and bulk compressibility, respectively, and $f$ is a body force. In order to derive these governing equations, we require several basic definitions.

For a cube of porous rock, an instantaneous change in confining stress yields a decrease of the mean normal stress by an amount $\Delta \sigma_{k k} / 3$. Consequently, a cube with undrained bulk modulus $K_{u}$ compacts isotropically by an amount $\Delta \sigma_{k k} / 3 K_{u}$. As a result, pore pressure increases by an amount $-B \delta \sigma_{k k} / 3$, where $B$ is Skempton's coefficient (e.g. Biot, 1941; Rice and Cleary, 1976; Roeloffs, 1988; Wang, 2000). The coseismic and subsequent postseismic pressure changes are often referred to as undrained and drained states with values of Poisson's ratio $\nu_{u}$ and $\nu$, respectively (e.g. Peltzer et al., 1998; Jonsson et al., 2003; Freed et al., 2006). In an undrained state (the coseismic period), forces are applied rapidly and the process is not in equilibrium. In contrast, if the porous solid is deformed slowly (during the postseismic period) fluids can flow and maintain equilibrium (Segall, 2010).

The constitutive behaviour of a porous elastic rock saturated with fluid was first described in detail by Biot (1962). Terzaghi (1943) had previously shown that for the first time that pore pressure $\left(P_{p}\right)$ has impact on the normal components of the stress tensor $\left(S_{i j} ; i=j\right)$ but not on the shear components $\left(S_{i j} ; i \neq j\right)$ according to equation

$$
\sigma_{i j}^{\prime}=S_{i j}-\delta_{i j} P_{p}
$$

where $\sigma_{i j}^{\prime}$ is the effective normal stress (the difference between applied stress and pore pressure) and $\delta_{i j}$ is the Kronecker delta function. Nur and Byerlee (1971) modified the Terzaghi equation to obtain the exact effective stress law

$$
\sigma_{i j}^{\prime}=S_{i j}-\delta_{i j} \alpha P_{p}
$$

where $\alpha$ is Biot coefficient

$$
\alpha=1-\frac{K_{b}}{K_{g}}
$$

and $K_{b}$ and $K_{g}$ are the rock bulk modulus and the individual solid grains' bulk modulus. For an elastic solid (with Lamé parameters $\lambda$ and $\mu$ ) and neglecting poroelasticity, the 


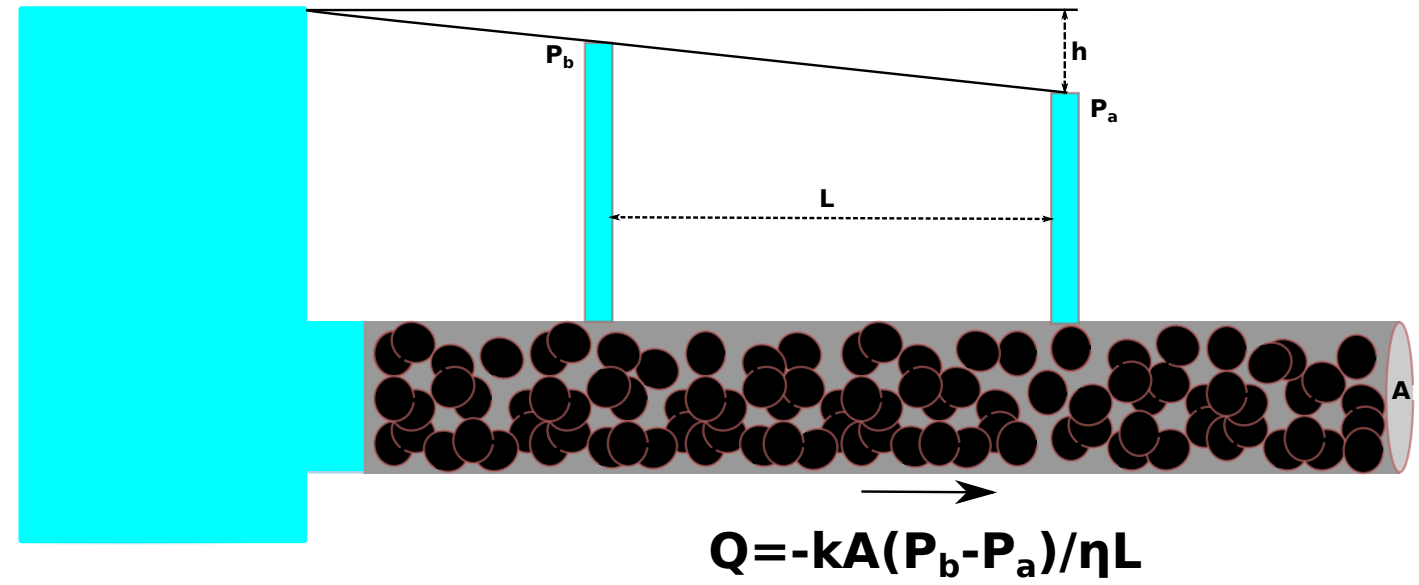

Figure 2.15: Fluid flow through porous medium formulted by Darcy's law.

stress and strain components are linearly proportional, such that

$$
\sigma_{i j}=\lambda \epsilon_{k k} \delta_{i j}+2 \mu \epsilon_{i j}
$$

when poroelasticity is considered, Equation 2.52 is modified to

$$
\sigma_{i j}=\lambda \epsilon_{k k} \delta_{i j}+2 \mu \epsilon_{i j}-\alpha \delta_{i j} P_{0}
$$

where

$$
P_{0}=\frac{1-2 \nu}{1+\nu} P_{p}
$$

and $\nu$ is Poisson's ratio (Nur and Byerlee, 1971; Zoback and Byerlee, 1975; Walls and Nur, 1979).

The strain-displacement relation and the equilibrium equations, respectively, are defined as follows

$$
\begin{array}{r}
\epsilon_{i j}=\frac{1}{2}\left(\frac{\partial d_{i}}{\partial x_{j}}+\frac{\partial d_{j}}{\partial x_{i}}\right) \\
\frac{\partial \sigma_{i j}}{\partial x_{j}}=f_{i}
\end{array}
$$

where $f_{i}$ and $d_{i}$ are the $i^{\text {th }}$ components of body force and displacement at position $x$.

Based on Darcy's law, the discharge rate $Q$ is proportional to the pressure drop between two points, the permeability $k$ and surface area $A$, and is inversely proportional to the distance $L$ between two points and the viscosity $\eta$ (Figure 2.15).

Accordingly, the volumetric flux density $q_{i}$ in the $i^{\text {th }}$ direction through a porous medium with permeability tensor $k_{i j}$ and density $\rho_{0}$ is obtained from Darcy's law

$$
q_{i}=-\rho_{0} \frac{k_{i j}}{\eta}\left(\frac{\partial P_{p}}{\partial x_{j}}-\rho_{0} g \delta_{3 j}\right)
$$


where $g$ is gravity, $\eta$ viscosity and $x_{j}$ the $j^{\text {th }}$ component of position (see Whitaker, 1986; Segall, 2010).

Conservation of fluid mass $(m)$ requires that

$$
\frac{\partial q_{i}}{\partial x_{j}}=\frac{\partial m}{\partial t}
$$

The strain-displacement relation (Equation 2.55), equilibrium conditions (Equation 2.56), Darcy's law (Equation 2.57), and conservation of fluid mass relation (Equation 2.58) are used along with the constitutive behaviour of a porous elastic solid (Equation 2.53) to derive the governing equations (for further details see Segall 2010 and Wang and Kümpel 2003).

$$
\begin{aligned}
\mu \nabla^{2} d_{i}+\frac{\mu}{1-2 \nu} \frac{\partial^{2} d_{j}}{\partial x_{i}} \partial x_{j}-\alpha \frac{\partial P_{p}}{\partial x_{j}} & =f_{i} \\
Q^{-1} \frac{\partial P_{p}}{\partial t}-\chi \nabla^{2} P_{p}+\alpha \frac{\partial}{\partial t} \nabla \cdot d & =0
\end{aligned}
$$

where bulk compressibility and Darcy conductivity are expressed as

$$
\begin{aligned}
Q^{-1} & =\frac{9}{2} \frac{\left(1-2 \nu_{u}\right)\left(\nu_{u}-\nu\right)}{(1-2 \nu)\left(1+\nu_{u}\right)^{2} \mu B^{2}} \\
\chi & =\frac{9}{2} \frac{\left(1-\nu_{u}\right)\left(\nu_{u}-\nu\right) D}{(1-\nu)\left(1+\nu_{u}\right)^{2} \mu B^{2}}
\end{aligned}
$$

Here $D$ is hydraulic diffusivity and $\nu_{u}$ and $\nu$ are the undrained and drained Poisson's ratios, respectively. The governing equations are expressed in vector form using the curl of the curl identity

$$
\nabla \times(\nabla \times d)=\nabla(\nabla \cdot d)-\nabla^{2} d
$$

Thus

$$
\begin{array}{r}
(\lambda+2 \mu) \nabla(\nabla \cdot \vec{d})-\mu \nabla \times(\nabla \times \vec{d})-\alpha \nabla P p=\vec{f} \\
Q^{-1} \frac{\partial P_{p}}{\partial t}-\chi \nabla^{2} P_{p}+\alpha \frac{\partial}{\partial t} \nabla \cdot \vec{d}=0
\end{array}
$$

where $\lambda=\frac{2 \mu \nu}{1-2 \nu}$.

\section{Poroelasticity's solutions}

Wang and Kümpel (2003) suggested a method for numerical computation of the governing equations 2.64 and 2.65 in a multi-layered poroelastic medium, using the LaplaceHankel transform of the solution vectors (Equation 2.66). For displacements of interior interfaces, the pore pressure and traction on both sides of the layer are equal. The top and bottom of the domain are assumed to be free surfaces with zero pressure changes and traction; the boundary conditions are satisfied in the numerical solutions. Using 
cylindrical surface harmonics, the governing equations are transformed to a system of ordinary differential equations. The Hankel transform for displacement is expressed in terms of cylindrical surface harmonics by

$$
d(z, r, \theta, s)=\sum_{m} \int_{0}^{\infty}\left[U_{m}(z, s) Z_{k}^{m}(r, \theta)+V_{m}(z, s) R_{k}^{m}(r, \theta)+W_{m}(z, s) T_{k}^{m}(r, \theta)\right] k d k
$$

where the cylindrical surface harmonics are

$$
\begin{aligned}
& Z_{k}^{m}(r, \theta)=e_{z} Y_{k}^{m}(r, \theta) \\
& R_{k}^{m}(r, \theta)=e_{r} \frac{1}{k} \frac{\partial}{\partial r} Y_{k}^{m}(r, \theta)+e_{\theta} \frac{1}{k r} \frac{\partial}{\partial \theta} Y_{k}^{m}(r, \theta) \\
& T_{k}^{m}(r, \theta)=e_{r} \frac{1}{k r} \frac{\partial}{\partial \theta} Y_{k}^{m}(r, \theta)-e_{\theta} \frac{1}{k} \frac{\partial}{\partial r} Y_{k}^{m}(r, \theta)
\end{aligned}
$$

Here $Y_{k}^{m}$ represents a Bessel functions of degree $m$ multiplied by a trigonometric vector (Equation 2.70), $(z, r, \theta)$ are the components of the cylindrical coordinate system, $s$ is the Laplace parameter, $U_{m}, V_{m}$ and $W_{m}$ are the coefficients in approximating the solution vectors.

$$
Y_{k}^{m}(r, \theta)=J_{m}(k r)\left(\begin{array}{c}
\cos m \theta \\
\sin m \theta
\end{array}\right)
$$

Using cylindrical harmonics, the problem of poroelasticity is simplified to optimising the coefficients $U_{m}, V_{m}$ and $W_{m}$. For more details see Wang and Kümpel (2003).

\section{Literature review of poroelastic rebound models}

A gradual change in Poisson's ratio from undrained (coseismic) to drained (postseismic) values is the conventional way of explaining observed poroelastic rebound following earthquakes (e.g. Peltzer et al., 1998; Jonsson et al., 2003; Freed et al., 2006). The relations between undrained $\nu_{u}$ and drained $\nu$ values for Poisson's ratio are written as (Wang, 2000)

$$
\begin{aligned}
\nu & =\frac{3 \nu_{u}-\alpha B\left(1+\nu_{u}\right)}{3-2 \alpha B\left(1+\nu_{u}\right)} \\
\nu_{u} & =\frac{3 \nu+\alpha B(1-2 \nu)}{3-\alpha B(1-2 \nu)}
\end{aligned}
$$

where $\alpha$ and $B$ are Biot and Skempton's coefficients.

Peltzer et al. (1998) used values of 0.31 and 0.27 for the undrained and drained Poisson's ratios to explain displacement observed across the Emerson Fault in a combination of afterslip and poroelastic rebound following the Landers earthquake. They showed that the poroelastic rebound effects with higher undrained Poisson's ratio of 0.35 account 
for the observed uplift signal in Homestead Valley.

Jonsson et al. (2003) observed subsidence within coseismic compressional zones and uplift within dilational zones correlated with water level changes. The signal associated with poroelastic rebound vanished within two months of the June 2000 sequence in the South Iceland Seismic Zone (SISZ). Freed et al. (2006) assessed the contribution of poroelastic rebound in the postseismic signal measured after the Denali, Alaska earthquake with undrained and drained values of 0.26 and 0.22 . They found largest contribution of poroelastic rebound near the Denali/Totschunda junction.

Assuming Poisson's ratio to be an isotopic material property is inappropriate in some cases, particularly along subduction zones due to the the contrast between stiff oceanic crust and compliant continental crust (Masterlark, 2003; Eberhart-Phillips and Stuart, 1992; Wald and Graves, 2001; Masterlark et al., 2001). Masterlark (2003) explained the delayed triggering effect from the $M_{w} 8.01995$ Jalisco-Colima Mexico subduction earthquake on a nearby $M_{w} 6.3$ rupture 63 days later due to a transient pore fluid pressure recovery. In their study, a 3D Finite Element Model was used to simulate the poroelastic postseismic displacement (see Section 2.2.6). They assigned different values of permeability to the oceanic and the continental crusts in order to account for the lag between the two events. In a similar study, Hughes et al. (2010) investigated stresscoupling between the $M_{w} 9.2$ Sumatra-Andaman and the $M_{w} 8.7$ Nias earthquakes in terms of transient fluid flow. Their model produced separate regimes of poroelastic rebound in the shallow forearc and volcanic arc and in the oceanic slab. The former dissipated within a month after the event. The migration of fluids within the oceanic crust led to the recovery of pore pressure changes three months after the main event which was coincident with the Nias earthquake.

Masterlark and Wang (2002) considered transient coupling between the Landers and Hector Mine earthquakes to be caused by upper-crust poroelastic rebound with a diffusivity of $0.01 \mathrm{~m}^{2} / \mathrm{s}$ and lower-crust viscous flow with a viscosity of $5 \times 10^{18} \mathrm{~Pa} \cdot \mathrm{s}$. Diffusivity $D$ controls the time scale of pore pressure recovery due to the migration of fluids and varies between $10^{-11}$ to $10^{5} \mathrm{~m}^{2} / \mathrm{s}$ (Roeloffs, 1996). Hydraulic diffusivity $D$ is expressed as (Rice and Cleary, 1976)

$$
D=\frac{2}{9} \frac{k}{\eta} B^{2} \mu \frac{(1-\nu)\left(1+\nu_{u}\right)^{2}}{\left(1-\nu_{u}\right)\left(\nu_{u}-\nu\right)}
$$

where $\eta$ and $k$ are viscosity and permeability. All other variables are as previously defined.

In Chapter 5, to model postseismic displacement following the Darfield earthquake (a non-subduction event), we neglect any lateral heterogeneity of poroelastic properties. 
We assume poroelastic properties only vary vertically within the continental crust. We use the multi-layered poroelastic rebound method appropriate for non-subduction earthquakes implemented by Wang and Kümpel (2003). This method enables us to consider vertical heterogeneity in a multi-layered poroelastic medium. Fully timedependent solutions can be estimated by this method which is another advantage over conventional methods that address only two end-members (undrained and drained). In contrast with the FEM, which approximates solutions with shape functions (see Section 2.2.6), poroelastic solutions are approximated by cylindrical surface harmonics (Wang and Kümpel, 2003).

\section{Example models of poroelasticity}

We present below the results of simulating poroelastic rebound using the Fortran codes implemented by Wang and Kümpel (2003). The ground displacement is computed in response to right-lateral slip of $5 \mathrm{~m}$ on a $90^{\circ}$ east striking vertical fault, with both length and width of $10 \mathrm{~km}$. We use P- and S-wave velocities from the national New Zealand velocity model in the Canterbury region (Eberhart-Phillips et al., 2010). Two values for hydraulic diffusivity are considered: $1 \mathrm{~m}^{2} / \mathrm{s}$ above depth of $1.75 \mathrm{~km}$ and $0.001 \mathrm{~m}^{2} / \mathrm{s}$ below depth of $1.75 \mathrm{~km}$. We set Skempton's and Biot coefficients to values of 0.5 and 0.6 , respectively. The cumulative east, north and vertical components of coseismic and postseismic displacement within 365 days of the simulated rupture are shown in Figure 2.16a and b, respectively. The flow of fluids induces subsidence and uplift (Figure 2.16b) in previously (during the coseismic period) compressional (+ sign Figure 2.16a) and dilational zones (- sign Figure 2.16a), respectively.

We also simulate poroelastic rebound within a step-over between two vertical dextral strike-slip faults with lengths and widths of $20 \mathrm{~km}$ and $10 \mathrm{~km}$, respectively, striking east to west and slipping by $2 \mathrm{~m}$. In both simulations, the vertical components of displacement due to coseismic and postseismic signals are similar in pattern and different in sign. In Figure 2.17c, we present the time series of poroelastic displacement for a point shown within the step-over. This shows that with the assumed poroelastic parameters, the east and vertical components for the point decay rapidly within approximately 60 and 90 days of the rupture, respectively. In Chapter 5, we apply a similar calculation to estimate the poroelastic rebound effect on the postseismic ground displacement following the Darfield earthquake . 

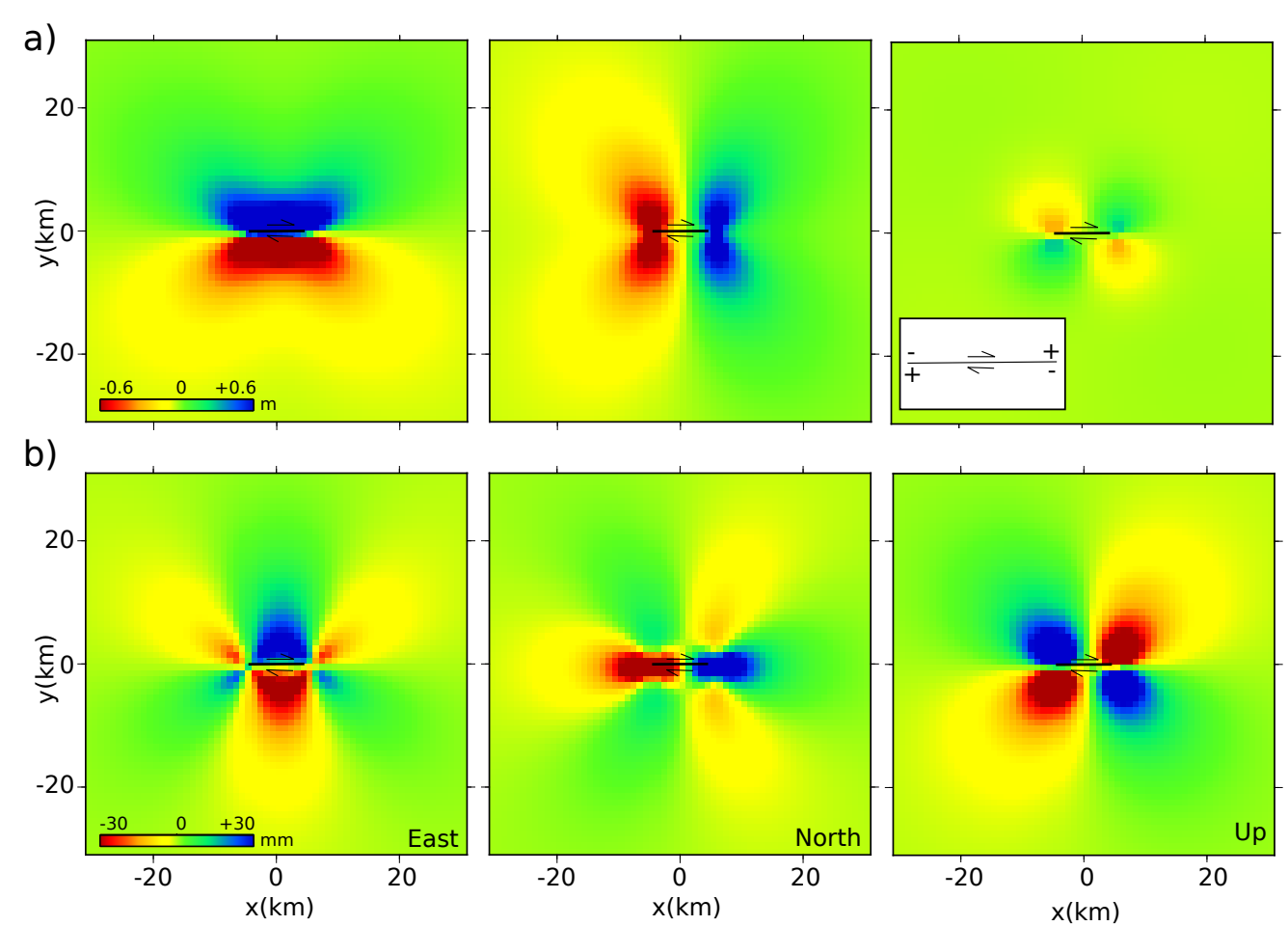

Figure 2.16: Simulated east, north and vertical components of a) Coseismic and b) Accumulated postseismic displacement within 365 days of the simulated rupture due right-lateral slip of $5 \mathrm{~m}$ on a vertical fault with the length and width of $10 \mathrm{~km}$. Red colours indicate movement away and blue colours indicate movement towards the direction. Dilational and compressional zones are illustrated by minus and positive signs, respectively. 

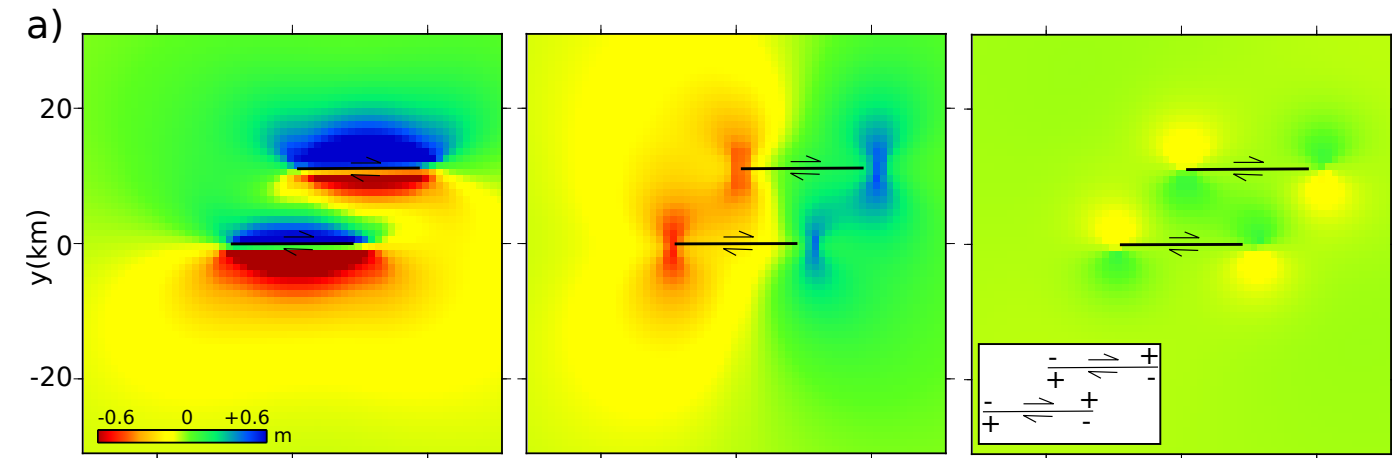

b)
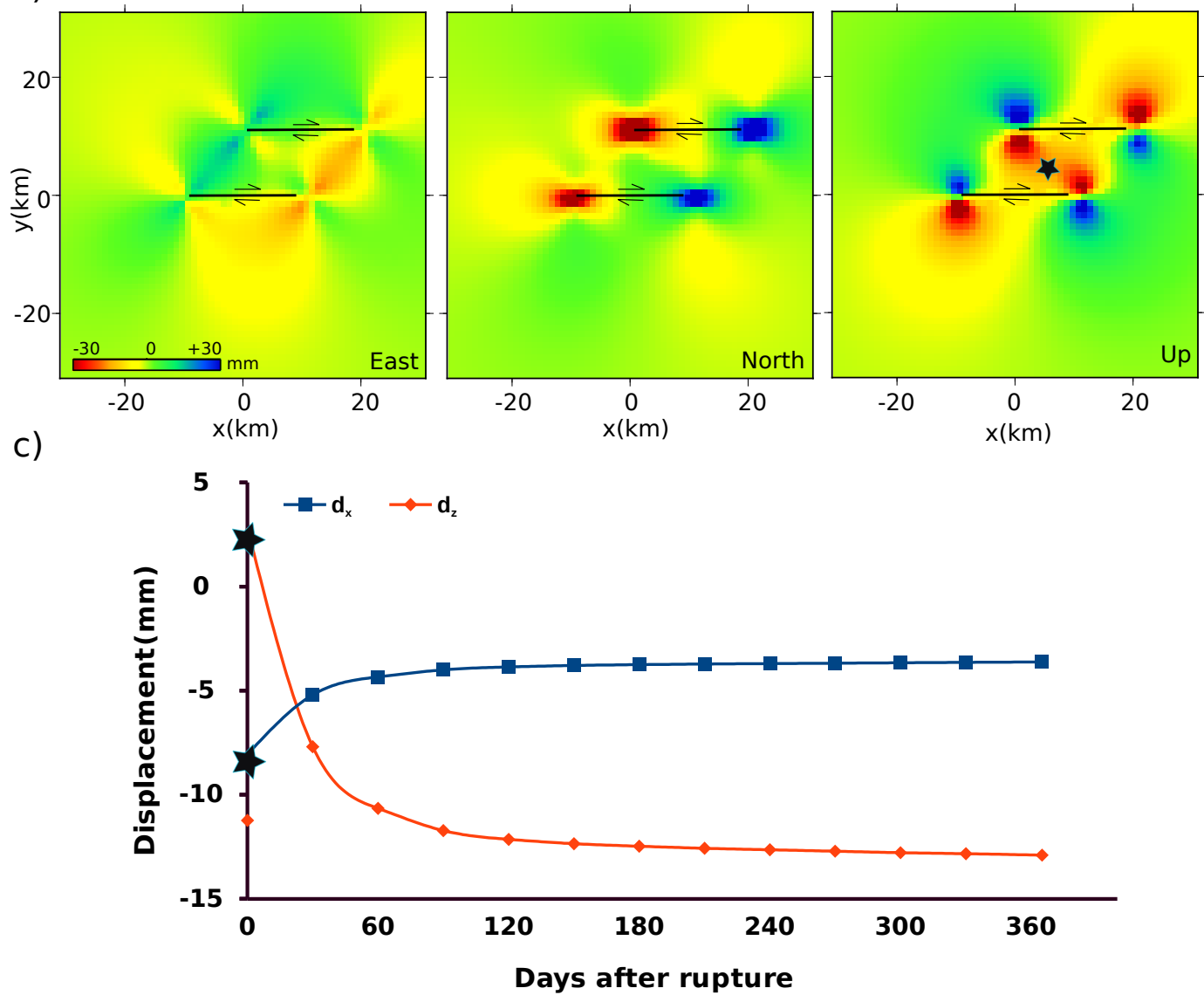

Figure 2.17: Simulated east, north and vertical components of a) Coseismic and b) Accumulated postseismic displacement within 365 days of the simulated rupture on a step over between two right-lateral faults with lengths and widths of 20 and $10 \mathrm{~km}$, respectively, and slip of $2 \mathrm{~m}$. Red colours indicate movement away and blue colours indicate movement towards the direction. Dilational and compressional zones are illustrated by minus and positive signs, respectively. c) Time series of east (blue) and vertical (red) components of displacement for every 30 days calculated from simulated poroelastic rebound.

\subsubsection{Shallow fault zone dilatancy recovery}

Static and dynamic stress changes after earthquakes cause the process of dilatancy during the coseismic period and the consequent post-recovery (Nur, 1971; Scholz, 1974; Vidale and Li, 2003). Dilatancy and its recovery alter the velocity of seismic waves 
after an earthquake and during postseismic period when the fault zone contracts (Vidale and Li, 2003; Schaff and Beroza, 2004; Peng and Ben-Zion, 2006; Reyners et al., 2014). Fialko et al. (2005) observed significant shallow slip deficits associated with the 2003 Bam earthquake in Iran. Subsequently, Fielding et al. (2009) explained this phenomenon to be the result of very shallow fault zone dilatancy recovery. Postseismic displacement due to viscoelastic relaxation in the lower crust/upper mantle, afterslip and poroelastic rebound in the upper crust often dominates the displacement field from dilatancy recovery. For the first time, Fielding et al. (2009) geodetically observed the subsidence signal corresponding to the process of dilatancy recovery 3.5 years after the 2003 Bam earthquake in Iran. To model the subsidence signal due to the contracting fault zone, an array of Mogi point sources are assumed on a fault surface (Figure 2.18). This method simultaneously allows the fault surface to slip and contract. Note that, Fielding et al. (2009) believes more continuous contracting volume source is required to explain the physics of dilatancy recovery. For the purpose of efficiency and simplicity, we use the same method in order to understand the process of dilatancy recovery within the top $4 \mathrm{~km}$ of the Darfield faults.

The ground displacement resulting from a combination of slip and volumetric displacement on a buried fault is linearly related to the slip components $\left(s_{s}\right.$ and $\left.s_{d}\right)$ and volume change $(\Delta v)$. This means that we can express the observation equation relating the observed ground displacement $(d)$ and the parameters of interest $(s)$ as follows

$$
d=G s+\epsilon=\left[\begin{array}{ll}
G_{f} & G_{v}
\end{array}\right]\left[\begin{array}{c}
s_{s} \\
s_{d} \\
\Delta v
\end{array}\right]+\epsilon
$$

where $G$ is the combined design matrix, $G_{f}$ and $G_{v}$ are functions of dislocation model and Mogi point source parameters (Okada, 1985; Mogi, 1958), and $\epsilon$ is the residual.

If $X, Y$ and $D$ are the east, north coordinates and depth of the point source with respect to the ground location and $R=\sqrt{x^{2}+y^{2}+d^{2}}$, then three-dimensional ground displacement induced by a Mogi point source is given by

$$
d_{v}=\frac{\nu-1}{\pi R^{3}}\left[\begin{array}{l}
X \\
Y \\
D
\end{array}\right] \Delta v+\epsilon_{v}=G_{v} \Delta v+\epsilon_{v}
$$

where $\nu$ is Poisson's ratio. In order to solve two components of afterslip on fault planes as well as the volume change, we use the linear least squares approach to invert the displacement signal. Inversion of Equation 2.74 is still an ill-posed problem and 
requires spatial smoothing. During the inversion, the following function is minimised

$$
f=\|d-G s\|^{2}+\gamma^{-2}\left\|\nabla^{2} s\right\|^{2}
$$

$\nabla^{2}$ is the approximation of the Laplacian operator. $\gamma$ determines the trade-off between misfits and smoothness of parameters. A value between spatially smooth and rough extremes is chosen (e.g. Beavan et al., 2012a) for hyperparameter $\gamma$, which minimises the misfit with observations and the smoothness.

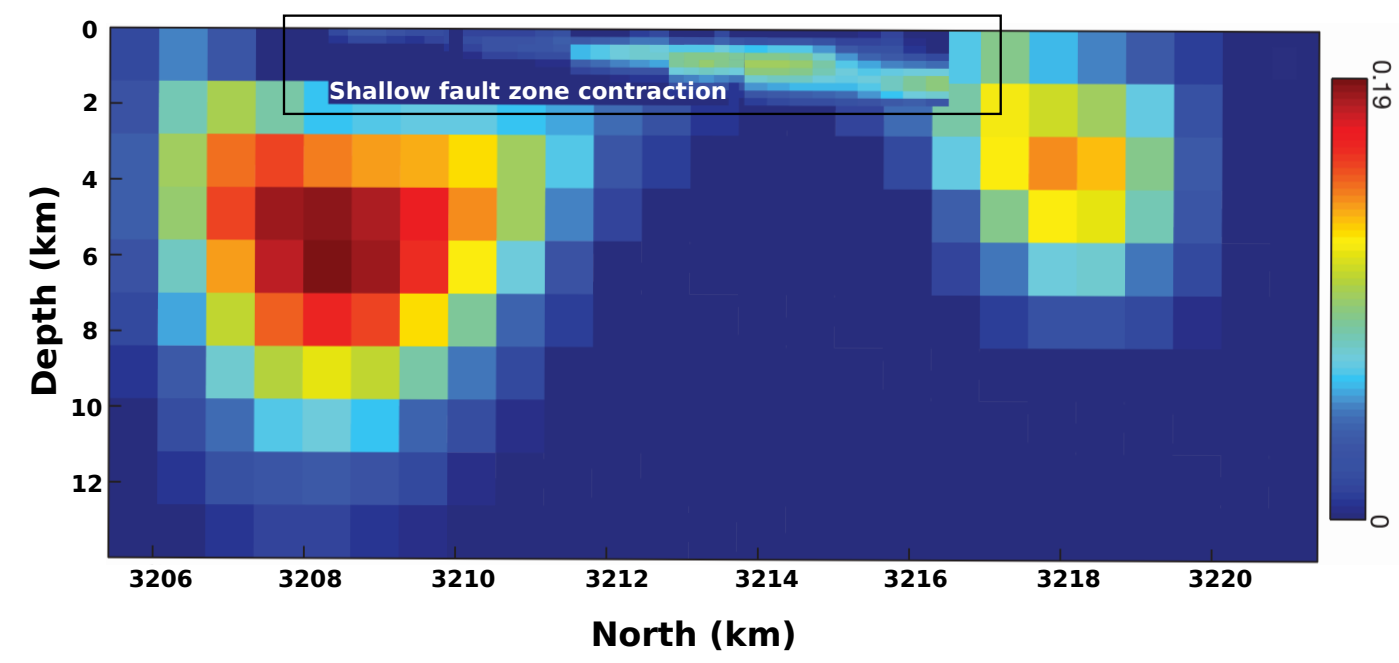

Figure 2.18: An array of regularly space contracting Mogi point sources in the shallow fault zone along with afterslip mechanism after the 200 Bam earthquake in Iran (Figure4; Fielding et al., 2009).

\subsubsection{Numerical computation using the Finite Element Method (FEM)}

The FEM is applied for modelling a structure by using finite number of interconnected elements (see Strang and Fix, 1973; Brenner and Carstensen, 2004). For complex structures, analytical solutions for a Boundary Value Problem (BVP) often do not exist.

As the name suggests, FEM begins with discretising the continuum into finite elements. For three-dimensional (3D) problems, tetrahedral and hexahedral (or brick) elements are widely used. Solving the BVPs using the FEM consists of: 1) forming the weak statement of the BVP; 2) approximating the weak form with the use of finite element functions (Hughes, 2012). A weak form is an integral function which is simpler to solve than the strong form of Partial Differential Equations (PDEs).

Using the FEM, element equations are converted to their corresponding matrix forms using variational or weighted residual methods (see Reddy, 1989, 1993). One of the 
primary sub-groups of the weighted residual methods is the Galerkin method by which weak forms of the element equations are generated (see Nayroles et al., 1992; Belytschko et al., 1994; Lu et al., 1994).

For a function $f$, the general weak form over the domain $\Omega$ is written as follows

$$
\int_{\Omega} w f d \Omega=0
$$

where $w$ is a weight function. The integral equations are solved using integration by parts. We approximate dependent variables (e.g. $u$ at position $x$ and time $t$ ) by their corresponding shape functions as expressed by

$$
u(x, t)=\sum_{i=1}^{n} \phi_{i}(x) u^{i}(t)=\Phi^{T} U
$$

where $\phi_{i}(x)$ are shape functions. The weighted residual formulation is applicable to a wide variety of BVPs. In the Galerkin method, we use the same weights for the dependent variables and the weak forms $(w \equiv \phi)$. There are other methods classified under weighted residual formulation such as techniques of collocation, sub-domain and least squares (e.g. Ackroyd, 1986; Reddy, 1989), but we do not discuss such techniques here.

To convert the element functions using the weak forms, we use the form below

$$
\begin{gathered}
{\left[\begin{array}{c}
f_{1} \\
f_{2} \\
\vdots \\
f_{n}
\end{array}\right]=\left[\begin{array}{cccc}
K_{11} & k_{12} & \cdots & k_{1 n} \\
K_{21} & k_{22} & \cdots & k_{2 n} \\
\vdots & & & \vdots \\
K_{n 1} & k_{n 2} & \cdots & k_{n n}
\end{array}\right]} \\
\{f\}=\{K\}\{u\}
\end{gathered}
$$

To illustrate this, we discuss the steps required for a simple heat transfer BVP by Equation 2.80

$$
\begin{aligned}
& f: \frac{\partial}{\partial x}\left(k_{x} \frac{\partial T}{\partial x}\right)+\frac{\partial}{\partial y}\left(k_{y} \frac{\partial T}{\partial y}\right)+\frac{\partial}{\partial z}\left(k_{z} \frac{\partial T}{\partial z}\right)-\frac{\partial T}{\partial t}=0 ; G=-\frac{\partial T}{\partial t} \\
& T=T_{b}: S 1 \\
& \left(k_{x} \frac{\partial T}{\partial x}\right) n_{1}+\left(k_{y} \frac{\partial T}{\partial y}\right) n_{2}+\left(k_{z} \frac{\partial T}{\partial z}\right) n_{3}+q=0: S 2 \\
& \left(k_{x} \frac{\partial T}{\partial x}\right) n_{1}+\left(k_{y} \frac{\partial T}{\partial y}\right) n_{2}+\left(k_{z} \frac{\partial T}{\partial z}\right) n_{3}+h\left(T-T_{a}\right)=0: S 3
\end{aligned}
$$

Here $T$ is the unknown temperature, $q$ heat flux, $h$ heat transfer coefficient, $n$ surface normal and $k$ the thermal conductivity. $S 1, S 2$ and $S 3$ are the boundaries shown in Figure 2.19. 


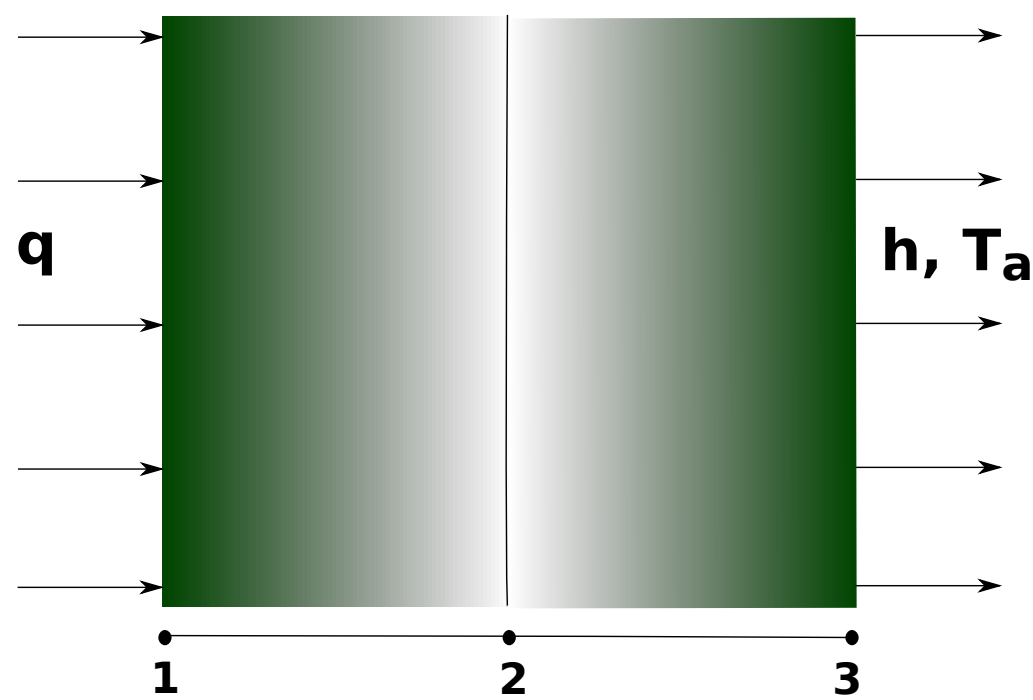

Figure 2.19: Heat transfer through two materials with three interfaces

The weak form of Equation 2.80 is achieved from Equation 2.77

$$
\int_{\Omega} w\left\{\frac{\partial}{\partial x}\left(k_{x} \frac{\partial T}{\partial x}\right)+\frac{\partial}{\partial y}\left(k_{y} \frac{\partial T}{\partial y}\right)+\frac{\partial}{\partial z}\left(k_{z} \frac{\partial T}{\partial z}\right)+G\right\} d \Omega=0
$$

Green's lemma derived from the integration by parts for arbitrary functions $\alpha_{1}$ and $\alpha_{2}$ over a domain $\Omega$ and a boundary $S$ is

$$
\int_{\Omega} \alpha_{1} \frac{\partial \alpha_{2}}{\partial x_{1}} d \Omega=-\int_{\Omega} \frac{\partial \alpha_{1}}{\partial x_{1}} \alpha_{2} d \Omega+\int_{S} \alpha_{1} \alpha_{2} n_{1} d S
$$

Using Green's lemma, Equation 2.81 is written in the form of

$$
\begin{aligned}
\int_{S} w\left(k_{x} \frac{\partial T}{\partial x} n_{1}+\right. & \left.k_{y} \frac{\partial T}{\partial y} n_{2}+k_{z} \frac{\partial T}{\partial z} n_{3}\right) d S \\
& -\int_{\Omega}\left(\frac{\partial w}{\partial x} k_{x} \frac{\partial T}{\partial x}+\frac{\partial w}{\partial y} k_{y} \frac{\partial T}{\partial y}+\frac{\partial w}{\partial z} k_{z} \frac{\partial T}{\partial z}\right) d \Omega+\int_{\Omega_{e}} G w d \Omega
\end{aligned}
$$

Equation 2.83 is approximated using the boundary conditions in Equation 2.80 as well as the approximation of a dependent variable in Equation 2.78. Using the Galerkin method, the weights for both dependent variables and weak forms are the same $(\phi)$.

$$
\begin{aligned}
-\int_{S} \phi_{k} q d S & +\int_{S} h \phi_{k} \phi_{i} T^{i} d S+\int_{S} h T_{a} \phi_{k} d S \\
& -\int_{\Omega}\left(k_{x} \frac{\partial \phi_{k}}{\partial x} \frac{\partial \phi_{i}}{\partial x}+k_{y} \frac{\partial \phi_{k}}{\partial y} \frac{\partial \phi_{i}}{\partial y}+k_{z} \frac{\partial \phi_{k}}{\partial z} \frac{\partial \phi_{i}}{\partial z}\right) T^{i} d \Omega+\int_{\Omega_{e}} G \phi_{k} d \Omega
\end{aligned}
$$

From Equation 2.84, components of matrices $f$ and $K$ in Equation 2.79 are extracted as follows

$$
\begin{array}{r}
K_{k i}=-\int_{\Omega}\left(k_{x} \frac{\partial \phi_{k}}{\partial x} \frac{\partial \phi_{i}}{\partial x}+k_{y} \frac{\partial \phi_{k}}{\partial y} \frac{\partial \phi_{i}}{\partial y}+k_{z} \frac{\partial \phi_{k}}{\partial z} \frac{\partial \phi_{i}}{\partial z}\right) d \Omega+\int_{S} h \phi_{k} \phi_{i} d S \\
f_{k}=-\int_{S} \phi_{k} q d S+\int_{S} h T_{a} \phi_{k} d S+\int_{\Omega_{e}} G \phi_{k} d \Omega
\end{array}
$$




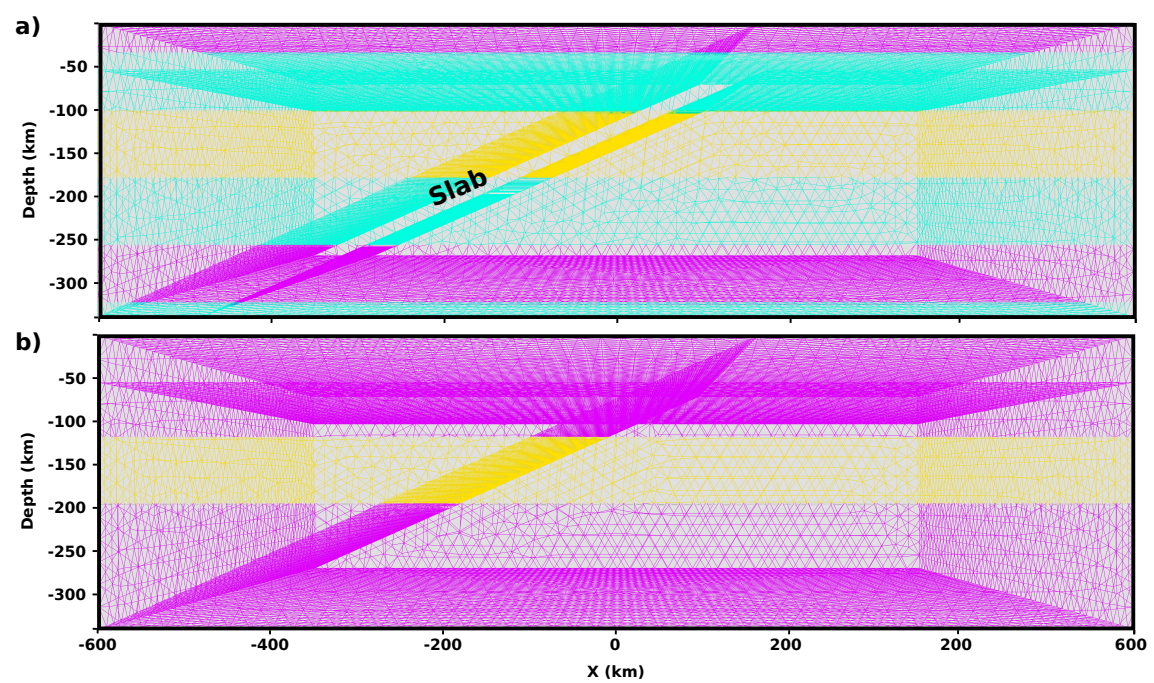

Figure 2.20: Tedrahedral mesh geometries generated in Trelis a) A geometry of an elasticviscoelastic medium for a subduction earthquake b) A simple geometry of an elasticviscoelastic medium

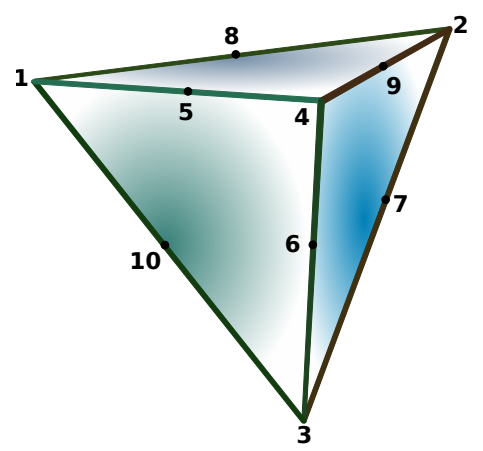

Figure 2.21: Quadratic tetrahedral element as a shape fucntion used in the Finite Element Method (FEM)

\section{Shape functions}

In Chapters 3 and 4, we discretise the 3D continuum into finite tetrahedral elements using a commercial software package Trelis ${ }^{2}$ (e.g. Figure 2.20). Here we briefly describe the shape functions associated with the quadratic tetrahedral element in three dimensions. The natural coordinates of a tetrahedral element are $\left(\xi_{1}, \xi_{2}, \xi_{3}, \xi_{4}\right)$. The $i^{\text {th }}$ component of the natural coordinates of the tetrahedron for point $P$ is the ratio between the volume encompassed by the point and the opposite face to the corner $i$, and the total volume (Figure 2.21). Thus, $\xi_{1}+\xi_{2}+\xi_{3}+\xi_{4}=1$ for each point. The shape functions for the quadratic tetrahedral element shown in Figure 2.21 are

\footnotetext{
${ }^{2}$ http://www.csimsoft.com/help/trelishelp.htm
} 


$$
\left[\begin{array}{l}
1 \\
x \\
y \\
z \\
u
\end{array}\right]=\left[\begin{array}{cccc}
1 & 1 & \cdots & 1 \\
x_{1} & x_{2} & \cdots & x_{10} \\
y_{1} & y_{2} & \cdots & y_{10} \\
z_{1} & z_{2} & \cdots & z_{10} \\
u_{1} & u_{2} & \cdots & u_{n}
\end{array}\right] *\left[\begin{array}{c}
\phi_{1} \\
\phi_{2} \\
\vdots \\
\vdots \\
\phi_{10}
\end{array}\right]
$$

Equation 2.86 shows how to calculate the coordinates of each point within a tetrahedron using the coordinates of the ten corners of the element and shape functions $\phi$. Similarly, an arbitrary dependent variable $u$ is approximated using the value of the dependent variable at ten corners and the shape functions. $\phi_{k}$ is a function of the natural coordinates of the tetrahedral element as below

$$
\begin{aligned}
\phi_{1} & =\xi_{1}\left(2 \xi_{1}-1\right) \\
\phi_{2} & =\xi_{2}\left(2 \xi_{2}-1\right) \\
\phi_{3} & =\xi_{3}\left(2 \xi_{3}-1\right) \\
\phi_{4} & =\xi_{4}\left(2 \xi_{4}-1\right) \\
\phi_{5} & =4 \xi_{1} \xi_{2} \\
\phi_{6} & =4 \xi_{2} \xi_{3} \\
\phi_{7} & =4 \xi_{3} \xi_{1} \\
\phi_{8} & =4 \xi_{1} \xi_{4} \\
\phi_{9} & =4 \xi_{2} \xi_{4} \\
\phi_{10} & =4 \xi_{3} \xi_{4}
\end{aligned}
$$




\title{
Chapter 3
}

\section{Postseismic displacement following the $M_{W}$ 7.8 Dusky Sound earthquake, Fiordland, New Zealand, and its effect on the $M_{\mathrm{W}} 7.1$ Darfield (Canterbury) earth- quake 14 months later}

\begin{abstract}
Continuous and campaign GPS observations reveal a broad postseismic displacement signal in the southern and central South Island of New Zealand in the months following the $\mathrm{M}_{\mathrm{W}}$ 7.8 Dusky Sound earthquake of 15 July 2009. The 2010-2012 Canterbury earthquake sequence that commenced with the $\mathrm{M}_{\mathrm{W}} 7.1$ Darfield (Canterbury) earthquake on 4 September 2010 and involved the fatal $\mathrm{M}_{\mathrm{W}} 6.2$ Christchurch earthquake and other damaging aftershocks occurred within the region of post-Dusky Sound displacement. This raises a fundamental question: did stress perturbations in the Canterbury region induced by the Dusky Sound earthquake 14 months earlier trigger or otherwise affect the Darfield earthquake and the protracted aftershock sequence that ensued? To answer this question, we use an iterative approach to quantify time-dependent afterslip following the Dusky Sound earthquake, and the coupled viscoelastic response to this afterslip and the original mainshock. The main patches of afterslip are inferred to have accommodated maximum cumulative slip exceeding 1 meter within 400 days of the mainshock. Our preferred model of afterslip involves oblique slip mainly at depths between 0-80 km in an elastic-viscoelastic medium with a Maxwell rheology and a viscosity higher than $10^{18} \mathrm{~Pa} \cdot \mathrm{s}$. After estimating the rheology and time-dependent afterslip, we calculate the Coulomb stress changes resolved on the faults involved in the Darfield event as well as the cumulative strain in the region. We estimate positive Coulomb stress changes of a few kilopascals immediately prior to the Darfield event due to both coseismic and postseismic mechanisms following the Dusky Sound earthquake. However, neither very small positive Coulomb stress changes of a few kilopascals nor
\end{abstract}


the estimated strain accumulation seems to have advanced the Darfield earthquake.

\subsection{Introduction}

The $\mathrm{M}_{\mathrm{W}} 7.8$ Dusky Sound earthquake of 15 July 2009 was the third-largest earthquake of that year globally ${ }^{1}$, and the largest New Zealand earthquake since the $\mathrm{M}_{\mathrm{W}} 7.8$ Hawke's Bay earthquake of $1931^{2}$. Despite its size, the earthquake caused little damage in southern New Zealand as a consequence of its occurrence in a little-populated area of the country and its south west-ward rupture direction (Fry et al., 2010; Beavan et al., 2010a). Moreover, its effects were quickly eclipsed by those of the 4 September 2010 Darfield (Canterbury) earthquake, which originated $37 \mathrm{~km}$ west of Christchurch, and the ensuing protracted aftershock sequence (Beavan et al., 2010b; Gledhill et al., 2010; Holden et al., 2011; Gledhill et al., 2011; Quigley et al., 2012). That sequence included the highly damaging Christchurch earthquake on 22 February 2011, which caused 185 fatalities (Holden, 2011; Fry et al., 2011; Bannister et al., 2011; Beavan et al., 2011; Kaiser et al., 2012). The spatial and temporal proximity of the events lead us to investigate whether the Canterbury earthquake sequence of 2010-2012 may have been triggered by the Dusky Sound earthquake.

In order to understand the interaction between earthquakes, and especially near- or farfield triggering of large earthquakes, it is important to consider the effects of postseismic as well as coseismic displacements. In principle, the effects of coseismic stress changes may be reinforced, diminished, or completely counteracted by postseismic processes such as the following:

- Stress transfer from the ductile lower crust and upper mantle to the brittle upper crust (e.g. Freed and Lin, 1998, 2001; Rydelek and Sacks, 2001; Freed, 2005; Pollitz et al., 2006a; Freed et al., 2007; Tabrez et al., 2008; He et al., 2011; Decriem and Árnadóttir, 2012);

- Changes in effective stress associated with pore fluid pressure redistribution (e.g. Cocco and Rice, 2002; Bosl and Nur, 2002; Masterlark and Wang, 2002; Piombo et al., 2005; Hughes et al., 2010, 2011); and

- Changes in stress caused by time-dependent frictional afterslip (e.g. Hearn et al., 2002a; Perfettini and Avouac, 2004; Perfettini and Ampuero, 2008; MurrayMoraleda and Simpson, 2009; Cattania et al., 2015).

Freed and Lin (1998) proposed that the first of these mechanisms caused lateral ex-

\footnotetext{
${ }^{1}$ http://www.emsc-csem.org/Earthquake and http://earthquake.usgs.gov/earthquakes/

${ }^{2}$ http://www.gns.cri.nz
} 
pansion of the region of positive coseismic Coulomb stresses following the $\mathrm{M}_{\mathrm{W}} 6.51971$ San Fernando earthquake. Similarly, Freed and Lin (2001) modelled viscoelastic stress transfer seven years following the $\mathrm{M}_{\mathrm{W}} 7.31992$ Landers earthquake and concluded that positive Coulomb stress changes of up to 0.1-0.2 MPa were produced at the hypocentre of the ensuing $\mathrm{M}_{\mathrm{W}} 7.11999$ Hector Mine earthquake. In comparison, Pollitz and Sacks (2002) calculated Coulomb stress increases of approximately 0.07 MPa on the Hector Mine fault surface due to postseismic relaxation. Hearn et al. (2002a) computed postseismic Coulomb stress changes of 0.1 MPa in response to modelled frictional afterslip in the time interval between the $\mathrm{M}_{\mathrm{W}} 7.61999$ İzmit and $\mathrm{M}_{\mathrm{W}}$ 7.2 Düzce earthquakes at the Düzce hypocentre. In that study, rheological models incorporating features such as a layered elastic structure at the Düzce hypocentre, a viscous shear zone, and viscoelastic lower crust in the Marmara region yielded greater Coulomb stress changes than those estimated from uniformly elastic frictional afterslip.

Coulomb stress changes in a poroelastic medium due to the redistribution of pore fluids after earthquakes were investigated by Piombo et al. (2005). They inferred from their simulation that the effect of pore fluid diffusion was to alter Coulomb stresses from initially negative values to significantly positive values at some locations. In the same way, pore-pressure recovery following the $\mathrm{M}_{\mathrm{W}} 9.12004$ Sumatra-Andaman earthquake has been interpreted to have clock-advanced the $M_{\mathrm{W}} 8.62005$ Nias earthquake (Hughes et al., 2010, 2011).

Based on these and other studies, and taking into account the large moment of the Dusky Sound earthquake, we describe and interpret the postseismic displacement that followed, and consider the effects of this displacement on stress and strain in the vicinity of the Darfield earthquake 14 months later. We first estimate afterslip and viscoelastic effects based on spatial and temporal patterns of displacement measured geodetically. Next, the stress changes and strain associated with these two displacement processes are computed at the position of the Darfield earthquake hypocentre, $\sim 600 \mathrm{~km}$ away, in order to examine the interaction between the two earthquakes. We presume the effects of pore fluid redistribution to be negligible at the distance from the Darfield earthquake $(\sim 600 \mathrm{~km})$. Distances of about 2.5 fault lengths (here $\sim 200 \mathrm{~km}$ ) are assumed to be affected by Coulomb stress changes induced by fluid distribution (Piombo et al., 2005).

\subsubsection{Tectonic setting}

We summarise the main features of present New Zealand plate tectonics from northernmost to southernmost as follows: 1) subduction of the Pacific plate along the Hiku- 
rangi Margin beneath the North Island (Barnes and Lépinay, 1997; Barnes et al., 1998; Reyners, 1998; Eberhart-Phillips and Reyners, 1999; Wallace et al., 2004; Barker et al., 2009); 2) a southward transition to transpressive slip along the Alpine Fault (Van Dissen and Yeats, 1991; Holt and Haines, 1995; Beavan et al., 1999; Norris and Cooper, 2001); and 3) eastward subduction of the Australian plate beneath the Pacific plate along the Puysegur Trench (Norris et al., 1990; Figure 4.6).

The fault surface associated with the Dusky Sound earthquake forms the plate boundary along the Puysegur subduction interface, dipping eastwards beneath southwestern New Zealand in the Fiordland region (Fry et al., 2010). Understanding the plate boundary, the geometry of the slab, the thickness of the elastic layer, and the heterogeneous lithospheric structure are of great importance in adapting our models for more complexities, as discussed further below.

Lebrun et al. (2000) located the Pacific-Australian plate boundary between the Alpine Fault and Puysegur Trench using bathymetric data. They also emphasised the difference between the northern subduction transition at the Hikurangi Margin and the southern transition at the Fiordland Margin. The northern transition occurs gradually via strain partitioning, whereas the southern transition involves abrupt changes in the strike direction and dip angle. This abrupt transition is associated with tearing of the subducting plate, which creates a crustal discontinuity at the Resolution Ridge System (Lebrun et al., 2000).

Subducting of the Australian plate beneath the Pacific plate in the Fiordland region accommodates the major component of plate motion. Near Doubtful Sound a sharp direction change in the strike of the subducting slab from $023^{\circ}$ to $040^{\circ}$ occurs from south to north (Figure 4.6 shown in the circle; Reyners and Webb, 2002). This bending of the subducting slab leads to a positive Bouguer gravity anomaly in the Fiordland region in a non isostatic manner (Malservisi et al., 2003).

Reyners et al. (1991) noted a contrast in dipping seismic zones from less steep in the south-west to near vertical north-east of the 4 June and 19 July 1988 Te Anau earthquakes. Down-dip tearing of the subduction zone was inferred from the orientation of the fault surfaces (Reyners et al., 1991), as previously suggested by Smith and Davey (1984). Smith and Davey (1984) concluded that the intermediate-depth events northeast of the Te Anau earthquake are situated on a rotated section of the Australian plate interface.

Eberhart-Phillips and Reyners (2001) imaged the Fiordland subduction zone using 3-D seismic tomography. They noted the major crustal features as follows: 1) low velocity 


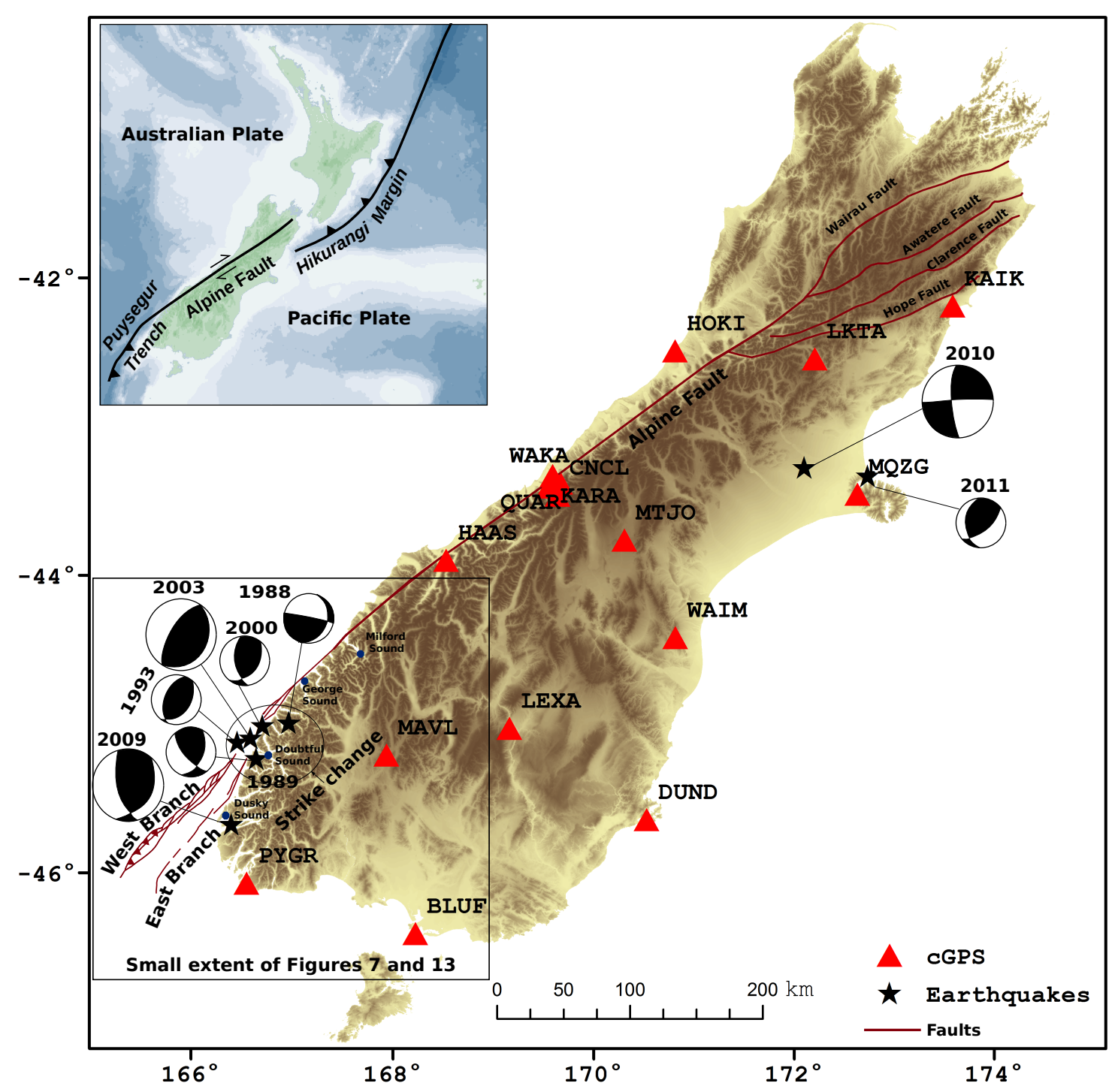

Figure 3.1: Tectonic setting of the Fiordland region and focal mechanisms of recent earthquakes, namely the 1988 Te Anau, 1989 Doubtful Sound, 1993 Secretary Island, 2000 Thompson Sound, 2003 Fiordland and 2009 Dusky Sound earthquakes, which were widely felt in the South Island, New Zealand. Subduction zone steepening and change of strike from south to north of Doubtful Sound are shown in the circle (Reyners and Webb, 2002).

within the upper $10 \mathrm{~km}$ beneath the Te Anau and Waiau basins, 2) Western Fiordland Orthogneiss with high velocity $\mathrm{Vp}$ and low ratio $\mathrm{Vp} / \mathrm{Vs}, 3$ ) high velocity Pacific mantle next to the deepest earthquakes, 4) high $\mathrm{Vp} / \mathrm{Vs}$ ratio where the subduction slab bends and expresses a distributed fracturing and finally 5) low velocity crustal root in the transition to the Alpine Fault, northern Fiordland. 


\subsubsection{Recent seismicity in southern New Zealand}

We here describe the characteristics of recent large earthquakes in the Fiordland region, namely the 1989 Doubtful Sound, 1993 Secretary Island, 2000 Thompson, 2003 Fiordland and 2009 Dusky Sound earthquakes, each of which was widely felt in the South Island. The focal mechanisms of these events are illustrated in Figure 4.6.

The $\mathrm{M}_{\mathrm{W}}$ 6.4 Doubtful Sound earthquake on 31 May 1989 involved oblique slip on a reverse fault (Anderson et al., 1993). The southeast-dipping nodal plane computed from first motion data was inferred to be the fault surface assuming that the mainshock occurred on the plate interface (Reyners and Webb, 2002). Four years later, the shallower $(22 \mathrm{~km}) \mathrm{M}_{\mathrm{W}} 6.8$ Secretary Island earthquake on 10 August 1993 initiated a rich aftershock sequence (Reyners and Webb, 2002). The rupture zone from this event seems to abut that of the earlier Doubtful Sound earthquake and lies within a zone of positive Coulomb stress changes, beneath the surface trace of the eastern strand of the Alpine Fault (Reyners and Webb, 2002). The results of body-wave inversion suggest that the Secretary Island earthquake involved nearly pure reverse faulting. The larger number of aftershocks associated with this earthquake than the Doubtful Sound event may reflect its shallower depth and depth-dependent variations of frictional properties at the plate interface (Reyners and Webb, 2002).

The Thompson Sound earthquake occurred on 1 November 2000. Robinson et al. (2003) relocated the mainshock and aftershock hypocentres using the double-difference method and demonstrated that the aftershocks define a west-dipping zone extending from $12 \mathrm{~km}$ to $24 \mathrm{~km}$.

Teleseismic body-wave inversions indicate that the Thompson Sound earthquake involved reverse slip with a subordinate left-lateral component. The cumulative Coulomb stress changes produced by a $\mathrm{M}_{\mathrm{W}} 6.7$ earthquake near Te Anau in 1988 and the 1989 Doubtful Sound and 1993 Secretary Island earthquakes were positive on the Thompson Sound rupture surface.

The $\mathrm{M}_{\mathrm{W}}$ 7.2 Fiordland earthquake on 21 August 2003 was the largest earthquake in southwest New Zealand in the 41 years prior to the Dusky Sound event (Reyners et al., 2003; McGinty and Robinson, 2007). Reyners et al. (2003) analysed the fault geometry of the Fiordland earthquake using campaign GPS measurements acquired shortly after the event between 29 August and 3 September 2003. Their model indicates that the earthquake was a shallow thrust event on the plate interface with a strike angle of $030^{\circ}$, a finding consistent with centroid moment tensor calculations. McGinty and Robinson (2007) noted that the aftershock distribution of the Fiordland earthquake did not define 
a planar rupture surface. They interpreted this event to have been triggered by the Secretary Island earthquake a decade earlier.

The largest earthquake to strike South Island of New Zealand in 80 years was the $\mathrm{M}_{\mathrm{W}}$ 7.8 Dusky Sound earthquake. Based on analysis of strong-motion records, Fry et al. (2010) inferred that rupture initiated at a depth of $38 \mathrm{~km}$ and proceeded upwards and southwestwards at a velocity of approximately $2.6 \mathrm{~km} / \mathrm{s}$.

Beavan et al. (2010a) modelled the fault geometry and slip distribution of the Dusky Sound earthquake using campaign and continuous GPS data and ascending and descending tracks of the ALOS/PALSAR radar satellite. Their results suggest a maximum slip of $\sim 6 \mathrm{~m}$ occurring on a curve-shaped patch of approximately $4000 \mathrm{~km}^{2}$ area, equivalent to a scalar moment of $\sim 4.0 \times 10^{20} \mathrm{~N} \cdot \mathrm{m}$. Li et al. (2011) also computed fault geometry and slip parameters and obtained similar results.

Despite its large size, the Dusky Sound earthquake's effects were eclipsed internationally by the very damaging earthquakes and tsunami that struck Tonga and Samoa in September 2009, and in the New Zealand public's mind by the much closer-to-home Darfield earthquake and then the damaging Christchurch earthquake. Approximately 14 months after the Dusky Sound earthquake, the Darfield earthquake nucleated 37 $\mathrm{km}$ west of Christchurch. This earthquake originated on the Charing Cross Thrust Fault (Holden et al., 2011; Gledhill et al., 2010, 2011) and propagated eastwards along the previously unknown Greendale Fault (Gledhill et al., 2011; Kaiser et al., 2012; Quigley et al., 2012). Following the mainshock, a prolonged aftershock sequence ensued, resulting with the 22 February 2011 Christchurch earthquake only $10 \mathrm{~km}$ from the central business district (Holden, 2011; Bannister et al., 2011; Beavan et al., 2011). That earthquake resulted in the loss of 185 lives and extensive damage to buildings and other infrastructure.

\subsection{Methods}

\subsubsection{Observations}

The observational data used in this chapter are 1) continuous GPS (cGPS) observations made at 16 sites in the southern to central South Island (locations and time series are represented in Figure 4.6 and Figure 3.4, respectively) ${ }^{3}$; 2) a campaign GPS dataset acquired shortly after the earthquake in August 2009 (Beavan et al., 2010a); and 3)

\footnotetext{
${ }^{3}$ Processed by Dr. Euan Smith
} 
Differential Interferometric Synthetic Aperture Radar (DInSAR) observations of postseismic displacement, acquired by PALSAR/ALOS satellite for the July 15-August 30 ascending path and the July 23-September 7 descending path (Beavan et al., 2010a).

Daily recordings of GPS data from the New Zealand network (GeoNet) were de-trended to determine mean secular velocities, as follows. First the longest period unaffected by regular or slow earthquakes prior to the Dusky Sound earthquake was identified by inspection. A weighted regression displacement against time was fitted. Weights were calculated as reciprocals of error estimates supplied by GeoNet. The residuals from the regression were then stacked to estimate short-term position changes (Figure 3.2; cf. spatial filtering; e.g. Wdowinski et al. 1997). This was removed from the time series and a seasonal effect was calculated using a 30 day moving average, which was also subtracted from the time series. The regression was then re-fitted to obtain velocities and uncertainty estimates. Mean residual variance reduction was $87 \%$.

The data from selected sites for the inter-earthquake period between the Dusky Sound and Darfield earthquakes (IEP; 416 days) was then detrended using the secular velocities. Sites from the eastern and central North Island were not considered because of ongoing slow earthquakes in the Hikurangi subduction zone (Douglas et al., 2005; McCaffrey et al., 2008; Wallace and Beavan, 2010). Co-and post-Dusky displacement was apparent on most South Island sites (Beavan et al. 2010). Three components of displacement were visible on sites within $200 \mathrm{~km}$ of the Dusky Sound centroid and used to represent the earthquake as a point source. The largest component was a westward movement, which was 3-5 times the southward component and $>7$ times the vertical component. Inspection suggested that the ongoing postseismic displacement was growing logarithmically. A model for the co- and post-Dusky displacement was fitted as follows. First an initial model for the displacement was fitted to an ensemble of sites, of the form

$$
d(t)=d_{0}+L \log \left(1+\frac{t}{c}\right)+e
$$

where $d$ is displacement, $t$ is time (days), $c$ is a timescale factor, $d_{0}$ is displacement at $t=0, L$ is the postseismic productivity and $e$ is an error, assumed Gaussian. For a set of observed displacements $d_{j}$ and times $t_{j}$ and a specified value of $c$, the maximum likelihood estimates of $d_{0}$ and $L$ can be found by (weighted) least squares regression of $d$ against $\log (1+t / c)$.

For the first fitting, $c$ was set to 1 day for all sites. Inspection showed that the model fit was not sensitive to the choice of $c$. However, for the stronger signals the best fit for a range of $\mathrm{c}$ values was given by $c=0.5$ or $c=1$ day. 

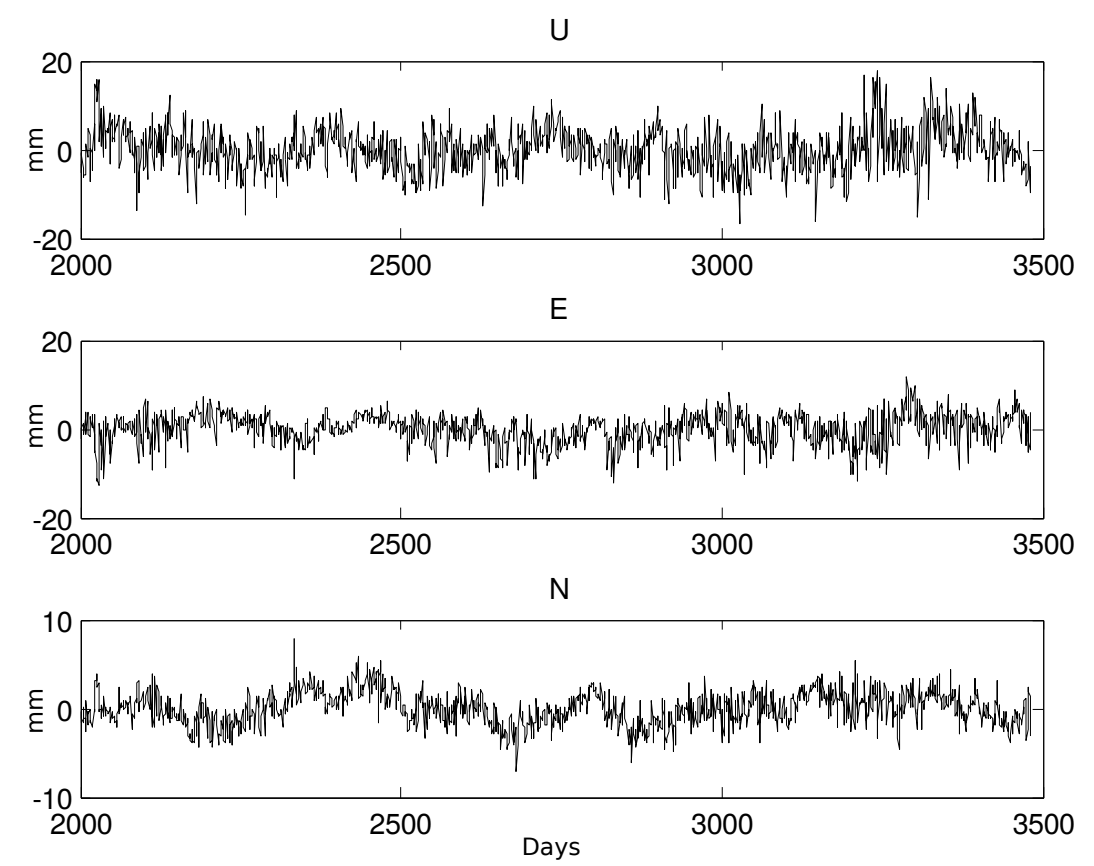

Figure 3.2: Stack of residuals pre-Dusky Sound earthquake from 22 sites.

Residuals from all but the nearest three sites were stacked for spatial filtering and a 30 point moving average of the filtered residuals was then calculated and subtracted. This method estimates the actual seasonal signal, including inter-seasonal variability (see Figure 3.3). Outliers amongst the residuals were down-weighted using Jeffreys' weighting, which downweights outliers in proportion to the probability that they arise from a central Gaussian distribution (Jeffreys, 1961). The fitted model represented by Equation 3.1 was then added back to the resulting time series. For the westward component, the best-fitting $c$ value was now 0.5 day at all sites except the nearest one (PYGR) where it was 1 day. For the final model fitting, $c=0.5$ day was adopted for all components at all sites. The final, overall Root Mean Square Error (RMSE) of the data-model was $1.1 \mathrm{~mm}$ (22 sites).

\subsubsection{Modelling time-dependent afterslip}

At time $t$, the elastic-viscoelastic displacement $\vec{d}$ in an arbitrary direction is presumed to be expressed by the superposition of responses to unit-step slip $(\vec{s})$ occurring at time $\tau<t$. Accordingly,

$$
\vec{d}(t)=\overrightarrow{d^{c}}(t)+\int_{0}^{t} G(t-\tau) \vec{v}(\tau) d \tau
$$



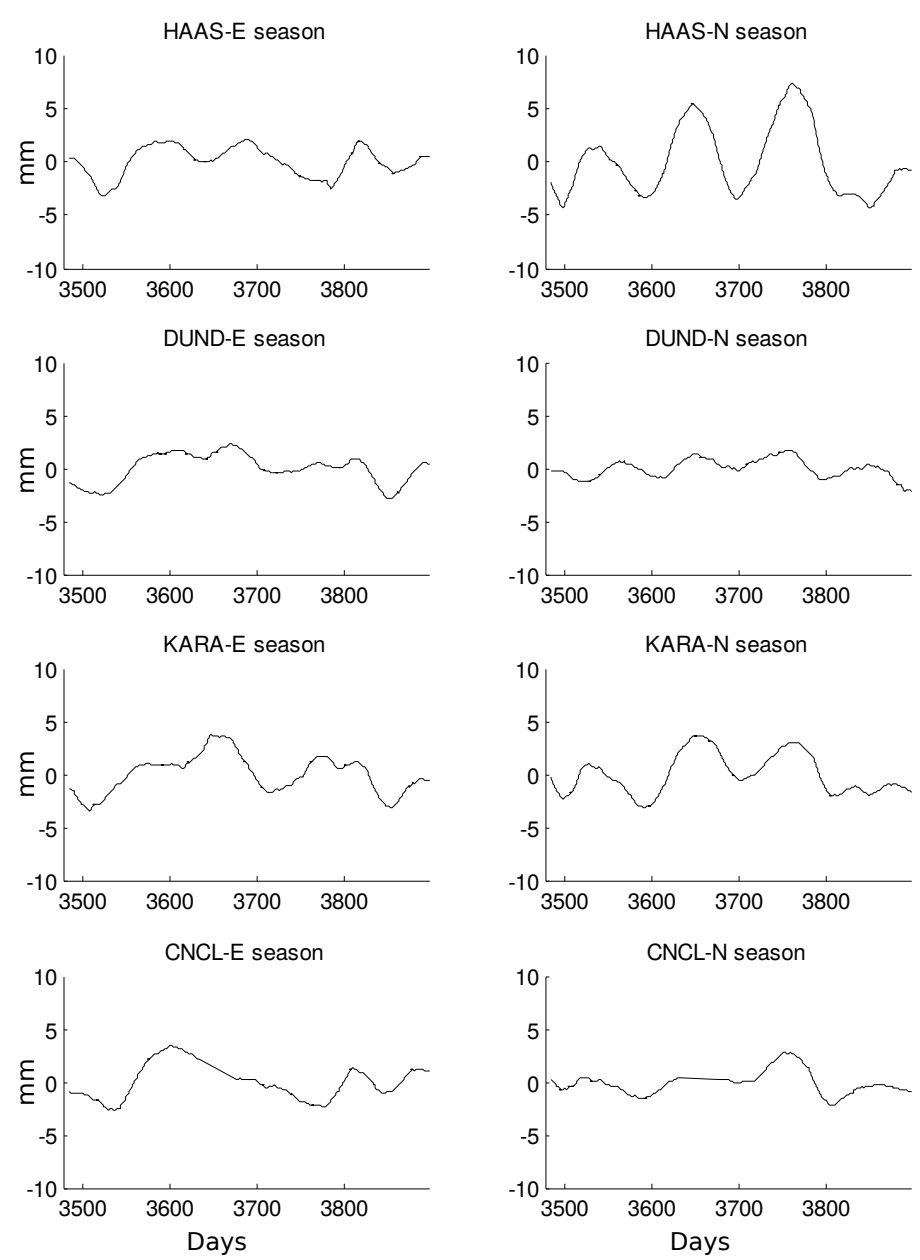

Figure 3.3: Selection of seasonal models, at HAAS, DUND KARA and CNCL sites, derived from 30 point moving averages as described in Section 3.2.1.

where $G$ is the matrix of corresponding elastic-viscoelastic Green's functions and $\vec{v}$ is the afterslip rate. By discretising the integral, we approximate Equation 3.2 as

$$
\vec{d}\left(t_{j}\right) \simeq \overrightarrow{d^{c}}\left(t_{j}\right)+\sum_{k=1}^{j-1} G\left(t_{j}-t_{k}\right) \vec{s}_{k}
$$

In Equations 3.2 and 3.3, the superscript $c$ indicates the viscoelastic displacement in response to coseismic stress changes.

At this point we have not discussed the viscoelastic response to time-dependent afterslip and we discuss how to account for this response in Section 3.2.3. We compute the viscoelastic response to the coseismic event and then subtract this from the observed displacement to leave $\vec{d}$ (the second term in Equations 3.2 and 3.3). The remainder is the elastic displacement due to time-dependent afterslip mechanism. Using elastic 

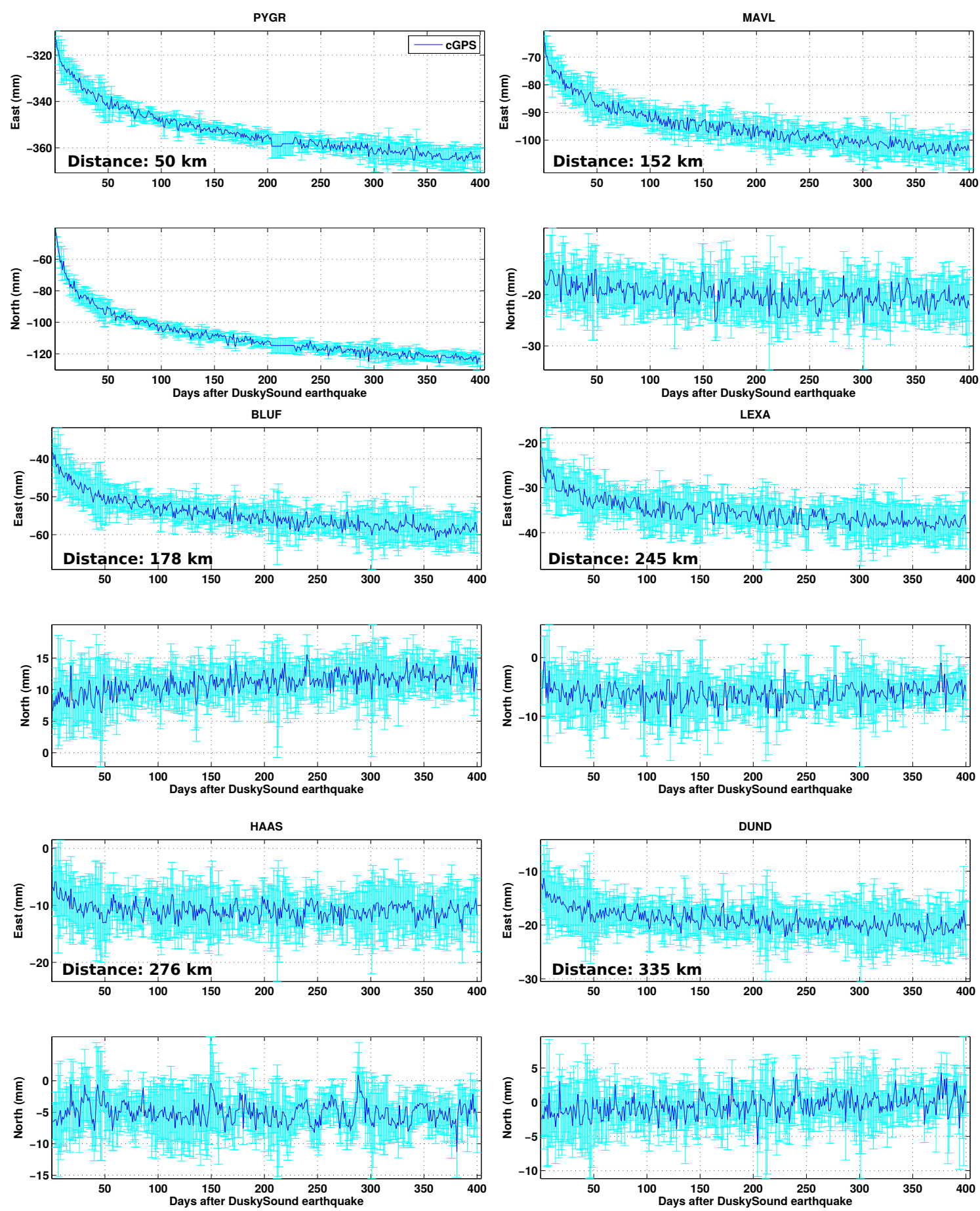

Figure 3.4: cGPS displacement observations (blue symbols) at PYGR, MAVL, BLUF, LEXA, HAAS, DUND sites. Locations of the sites are presented in Figure 4.6.

Green's functions $\left(G_{e}\right)$ Equation 3.3 can then be rewritten as follows

$$
\begin{aligned}
\vec{d}_{n \times 1}^{1} & =G_{e} \vec{s}_{m \times 1}^{1} \\
\vec{d}_{n \times 1}^{2} & =G_{e} \vec{s}_{m \times 1}^{1}+G_{e} \vec{s}_{m \times 1}^{2} \\
\vec{d}_{n \times 1}^{3} & =G_{e} \vec{s}_{m \times 1}^{1}+G_{e} \vec{s}_{m \times 1}^{2}+G_{e} \vec{s}_{m \times 1}^{3} \\
\quad & \\
\vec{d}_{n \times 1}^{T} & =G_{e} \vec{s}_{m \times 1}^{1}+G_{e} \vec{s}_{m \times 1}^{2}+\cdots \cdots \cdots+G_{e} \vec{s}_{m \times 1}^{T}
\end{aligned}
$$



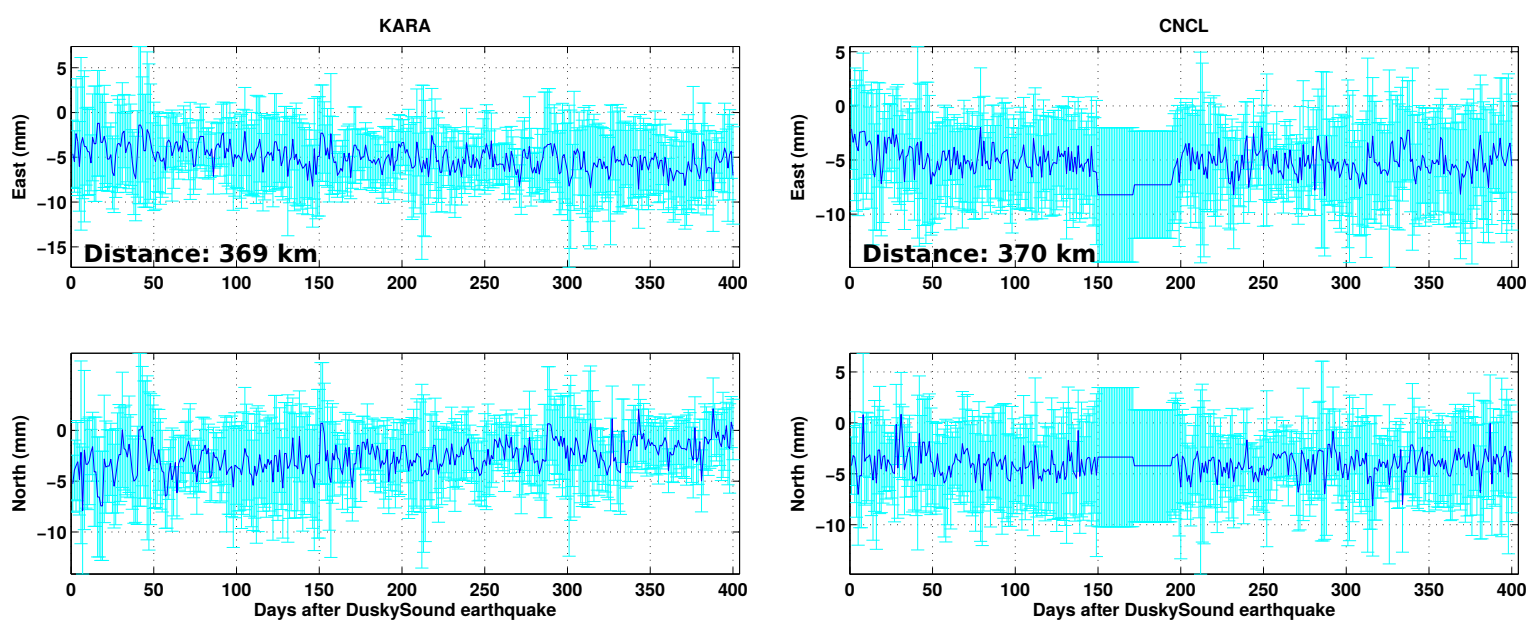

QUAR
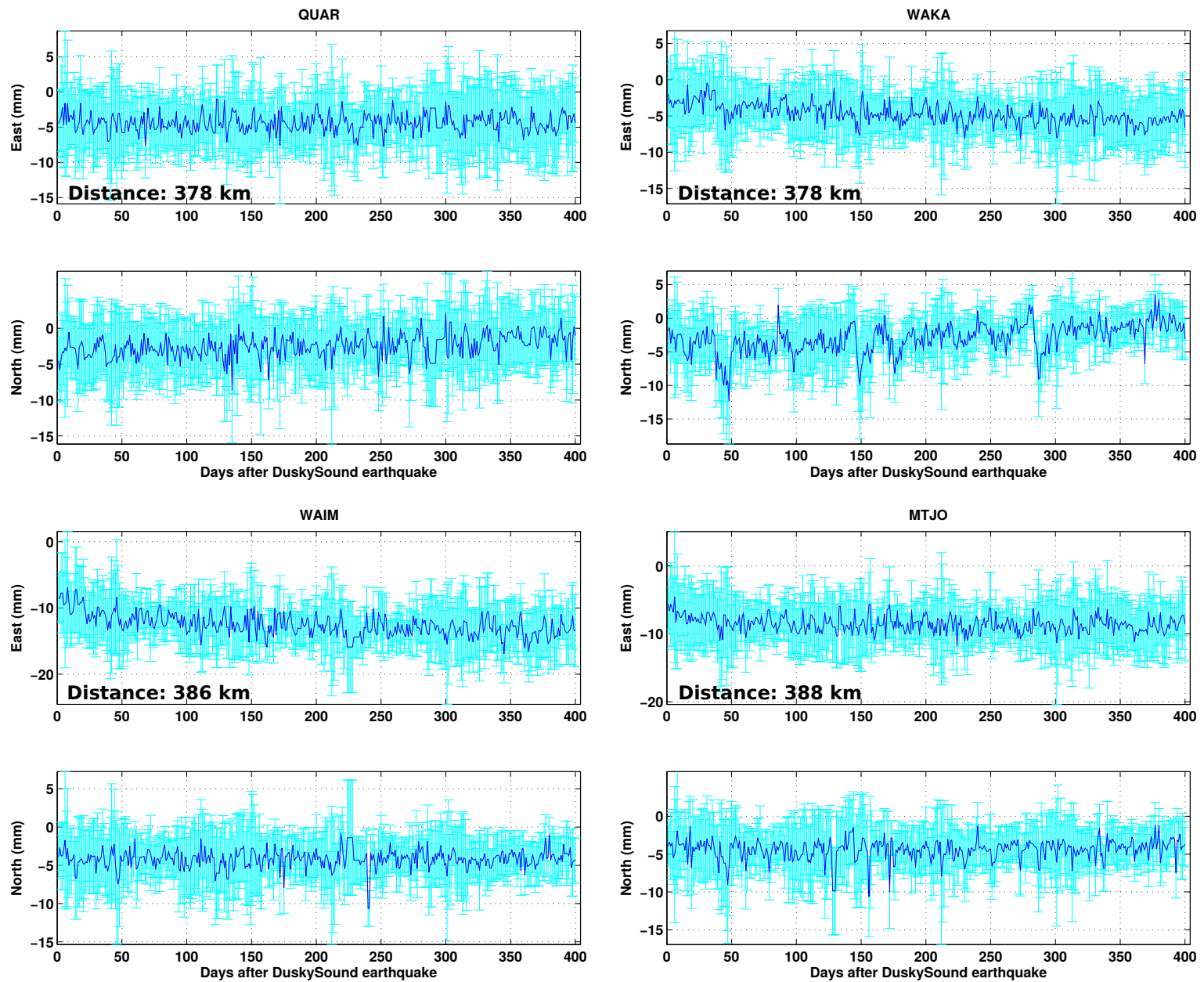

cGPS displacement observations (blue symbols) at KARA, CNCL, QUAR, WAKA, WAIM and MTJO sites. Locations of the sites are presented in Figure 4.6.

where $n$ is the number of displacement components, $m$ the number of slip components on the fault surface and $T$ the number of epochs. $G_{e}$ represents the elastic Green's functions with dimensions of $n \times m$. The displacement vector at epoch $T$ is written in terms of cumulative afterslip $\left(\vec{S}^{T}\right)$ as

$$
\vec{d}_{n \times 1}^{T}=G_{e} \vec{S}_{m \times 1}^{T}
$$



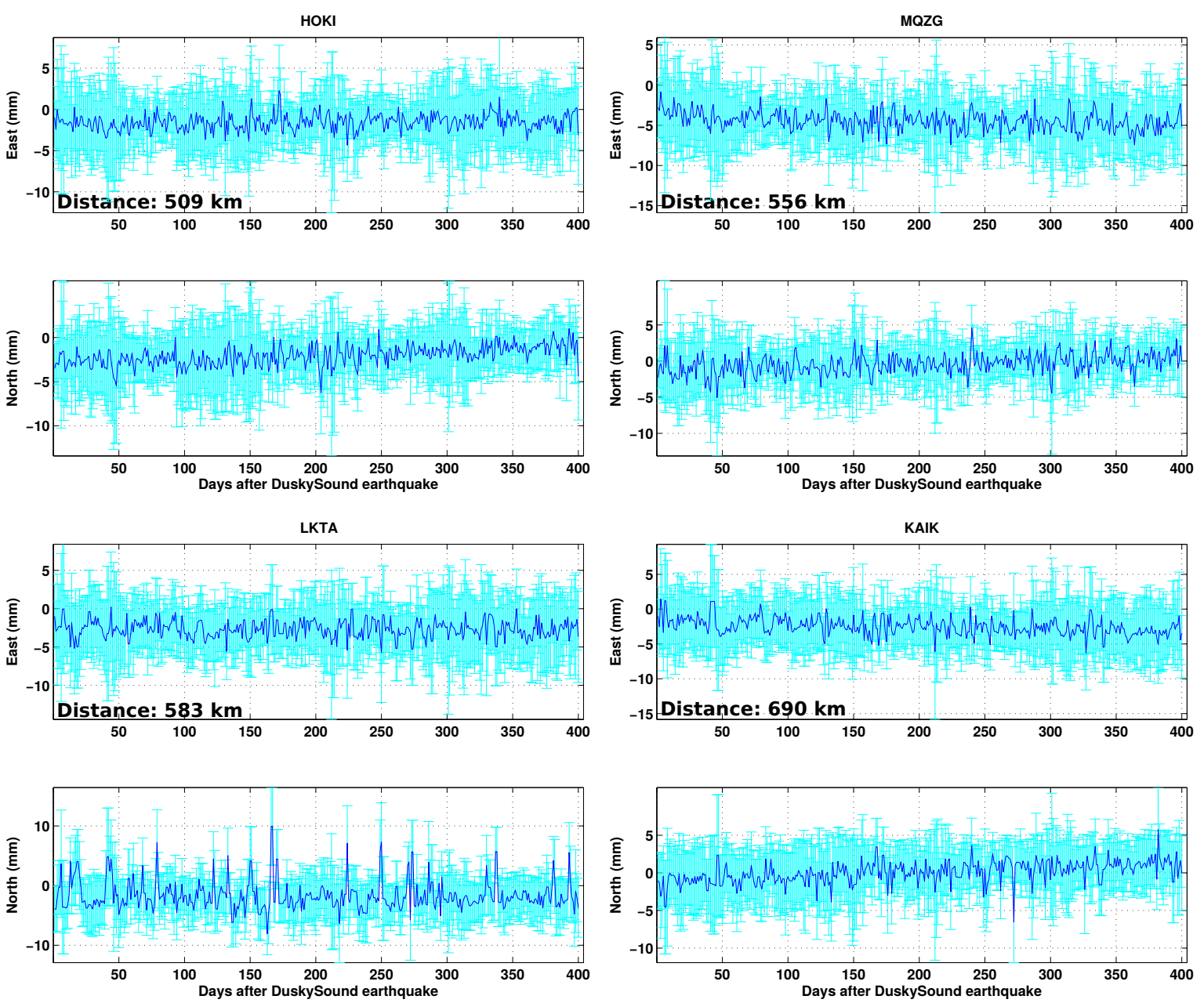

cGPS displacement observations (blue symbols) at HOKI, MQZG, LKTA and KAIK sites. Locations of the sites are presented in Figure 4.6.

Equation 3.5 is expressed in matrix form as

$$
D_{n \times T}=G_{e} \mathbf{S}_{m \times T}
$$

where $D$ and $\mathbf{S}$ are the time-dependent displacement and cumulative afterslip matrices, respectively.

The strategy underpinning our time-dependent afterslip calculations is based on a Singular Value Decomposition (SVD; see Forsythe and Moler, 1967) of the spatio-temporal displacement signal into a space function $U$ and a time function $V$ (Kositsky and Avouac, 2010). In other words, we represent the matrix $D$ consisting of displacement components measured at $T$ epochs as

$$
D=U \Lambda V^{\prime}
$$

where $U$ is an $n \times n$ matrix whose columns are the left singular vectors of $D, V$ is a $T \times T$ matrix whose columns are the right singular vectors of $D$, and $\Lambda$ is an $n \times T$ diagonal matrix containing the singular values of $D$. 
Given the linearity of the problem, the slip function with $m$ components is simply expressed as

$$
\mathbf{S}=G_{e}^{-1} U \Lambda V^{\prime}
$$

where the $n \times m$ matrix $G_{e}$ is calculated from elastic Green's functions relating the fault slip to the surface displacement (Okada, 1985). $G_{e}^{-1}$ refers to the regularised inverse of $G_{e}$.

The Principal Component Analysis-based Inversion Method (PCAIM) introduced by Kositsky and Avouac (2010), without considering the viscoelastic effects, simplifies the time-dependent inversion process to one in which the inversion step is applied only to the spatial function $(U)$ obtained by Singular Value Decomposition (SVD) and temporal information is retrieved from the time function $(V)$. In this method, temporally dense information such as continuous GPS or InSAR time series are used to decompose the displacement signal into spatial and time functions. Additionally, we can constrain the spatial pattern of fault slip $\left(U_{s}\right)$ using temporally sparse data such as InSAR or campaign GPS data ( $D_{\text {sparse }}$; Kositsky and Avouac, 2010). If $\mathbf{S}$ is written in terms of slip spatial and temporal functions:

$$
\mathbf{S}=U_{s} \Lambda_{s} V_{s}^{\prime}
$$

using Equation 3.6

$$
\begin{aligned}
D & =G_{e} U_{s} \Lambda_{s} V_{s}^{\prime} \\
V_{s}^{t} & =\Lambda_{s} V_{s}^{\prime}
\end{aligned}
$$

then

$$
D=G_{e} U_{s} V_{s}^{t}
$$

where $U_{s}, \Lambda_{s}$ and $V_{s}^{\prime}$ are the matrices of spatial, scaling and time functions of fault slip, respectively (' denotes matrix transpose). $V_{s}^{t}$ is expressed as the multiplication of the singular values of the slip matrix by the slip time function. If the campaign dataset spans the time interval between $t_{1}$ and $t_{2}$, the sparse constraint equation for a set of sparse observations is written as

$$
D_{\text {sparse }}=G_{e}\left[V_{s}^{t}\left(t_{2}\right)-V_{s}^{t}\left(t_{1}\right)\right] U_{s}
$$

The PCAIM method decomposes the observed displacement signals into components of decreasing singular value and thus of successively lower significance. Correspondingly, instead of using the full SVD representation of the displacements $D$, we can restrict the analysis to only the first $r$ components, in which case

$$
D_{r}=U_{r} \Lambda_{r} V_{r}^{\prime}
$$


Here $D_{r}$ is a filtered form of the full displacement matrix $D$, of the same dimensions $n \times T$; $U_{r}$ is of dimension $n \times r$, representing the first $r$ columns of $U ; \Lambda_{r}$ is of dimension $r \times r$, formed from the first $r$ rows and columns of $\Lambda$; and $V_{r}$ is of dimension $T \times r$ and represents the first $r$ columns of $V$. The optimal value of $r$ is chosen by minimising the misfit between the displacement signal $D$ and the filtered signal $D_{r}$ (Kositsky and Avouac, 2010). Filtering by single value truncation is performed assuming the single values are sorted from high to low.

\subsubsection{Modelling coupled viscoelastic rebound and afterslip}

Postseismic displacement following large earthquakes has generally been modelled in terms of three separate mechanisms: 1) short-term afterslip in the upper/lower crust on the mainshock fault surface (e.g. Savage and Church, 1974; Johnson et al., 2012; Wen et al., 2012a; Reddy et al., 2013); 2) poroelastic rebound (e.g. Booker, 1974; Rice and Cleary, 1976; Barbot and Fialko, 2010b; Lubis et al., 2013); or 3) long-term viscoelastic relaxation in the lower crust/upper mantle (e.g. Elsasser, 1967; Savage and Prescott, 1978; Pollitz et al., 2000; Pollitz, 2003b; Rousset et al., 2012b). Here, we allow for short-term time-dependent afterslip and longer-term viscoelastic rebound to both occur during the postseismic time interval in response to deviatoric stresses induced coseismically (Barbot and Fialko, 2010a).

A simple Maxwell rheology consisting of an elastic layer overlying a viscoelastic halfspace of uniform viscosity and hence uniform relaxation time is the most widely adopted representation of viscoelastic relaxation (e.g. Koseluk and Bischke, 1981; Pollitz and Dixon, 1998; Hao et al., 2012). Multiphase relaxation can be treated using either more complex rheologies, such as a bi-viscous Burger's body with two phases of viscoelastic creep (e.g. Pollitz, 2003b; Hetland and Hager, 2005) or a Standard Linear Solid (SLS) (e.g. Pollitz et al., 2000; Decriem and Árnadóttir, 2012), or by using different layerings, such as that consisting of a viscoelastic layer sandwiched between an elastic upper layer and a viscoelastic lower half-space (e.g. Hetland and Hager, 2005).

In several previous studies, other factors such as coupling between displacement and gravity field (e.g. Fernández and Rundle, 2004; Wang, 2005; Wang et al., 2006), lateral heterogeneity (e.g. Pollitz, 2003a), stratification (e.g. Hetland and Hager, 2005) and compressibility (e.g. Tanaka et al., 2006) have been considered in order to provide more realistic models. As a first attempt at examining the postseismic displacement induced by the Dusky Sound earthquake, we consider only simple Maxwell rheologies of various viscosities and a single bi-viscous Burger's body in an elastic-viscoelastic medium. In 
each case, we use a homogeneous elastic layer with a thickness of $45 \mathrm{~km}$, appropriate for this region based on the 3-D seismic velocity model of New Zealand (Eberhart-Phillips et al., 2010), overlying a viscoelastic half-space. This simple viscoelastic forward model is used in this chapter due to several limitations: 1) sparsity of the continuous GPS measurements in the central and southern South Island; 2) lack of campaign GPS measurements spanning the early postseismic interval excluding the coseismic signal; 3) lack of sufficient structural data. The fault surface is approximately inferred by Beavan et al. (2010a) from initial geodetic and seismic models (Fry et al., 2010); and 4) solving the problem in an inversion framework rather than forward modelling.

Our modelling strategy is illustrated schematically in Figure 3.5. To couple viscoelastic and afterslip models, we first compute the viscoelastic response to coseismic stress changes using the coseismic slip model of (Beavan et al. 2010a; $D_{\text {co-viscous }}$ ) and the numerical code PyLith (Aagaard et al., 2009, 2013, 2015). We subtract the viscoelastic response from the observations $\left(D_{\text {pseudo }}\right)$, to remove the viscoelastic response to the coseismic event. Next, after centering the time series of the displacement about zero, we invert the remaining signal using elastic Green's functions to extract the time-dependent afterslip using the decomposition technique described in the previous section. In doing so we must account for the fact that the stress changes associated with afterslip can themselves produce a viscoelastic response that has not yet been accounted for. Therefore, we re-estimate displacement time series as the response to the previously inferred time-dependent afterslip in an elastic-viscoelastic medium with the assumed rheology in PyLith. If the viscoelastic response to the previous afterslip model is significant enough, the displacement can vary from the previously calculated time series.

To address this, we perform an iterative calculation in which a correction term equal to the difference between observed and calculated displacements $D_{\text {correction }}=D-D_{\text {PyLith }}$ is added to the previous calculation of ground displacement to incorporate the viscoelastic response to afterslip. The lower the viscosity of the viscoelastic medium, the larger the viscoelastic response to afterslip becomes, meaning that more iterations are required for convergence. For reasons discussed below, the lowest viscosity used here is $5 \times 10^{18}$ $\mathrm{Pa} \cdot \mathrm{s}$ and even in this case only one iteration is required to fit the observations.

We note that using elastic Green's functions throughout the PCAIM inversion method does not allow us to incorporate the viscoelasic effects directly in our inversion. Alternatively, time-dependent numerical techniques such as Aagaard et al. (2009, 2013, 2015) or semi-analytical propagator matrix methods of Fukahata and Matsu'ura (2006) for instance, are used to estimate the viscoelastic Green's functions and thus to relate 


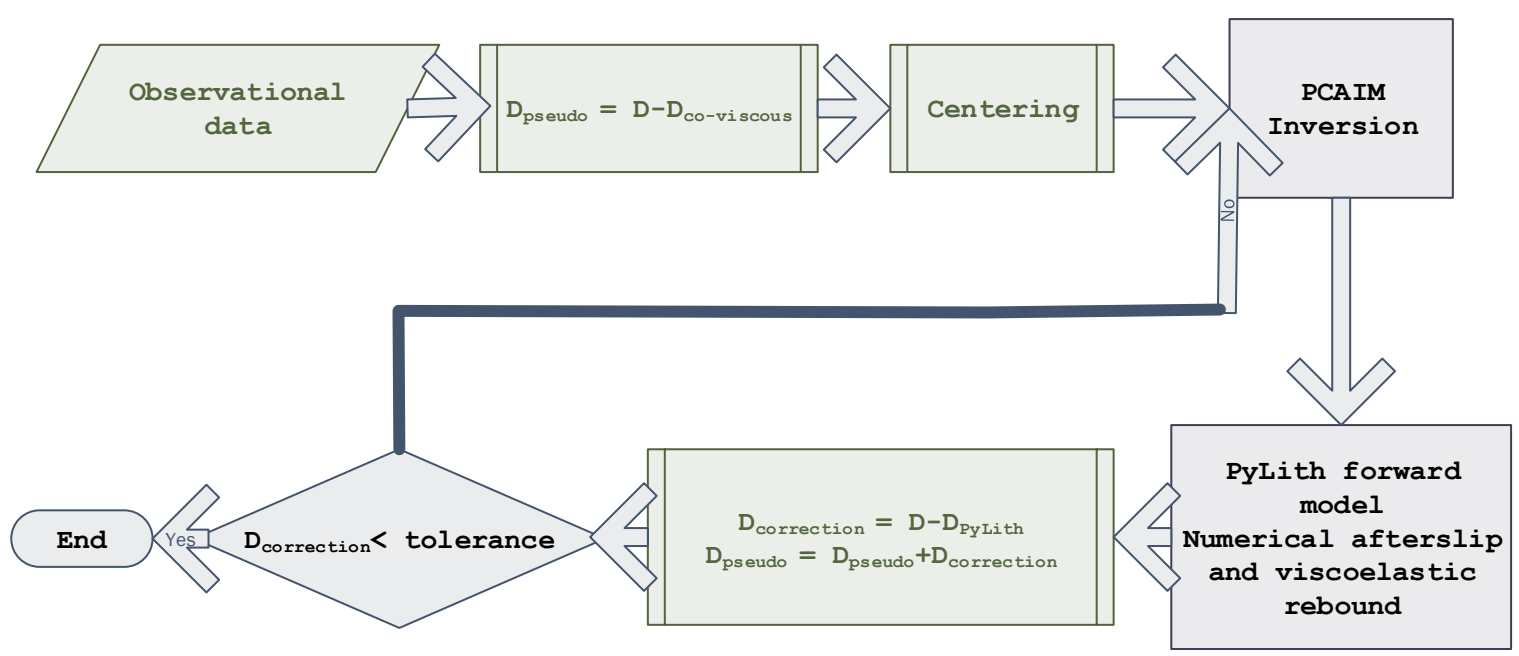

Figure 3.5: Flowchart illustrating the iterative approach used in order to couple timedependent afterslip and viscoelastic rebound.

slip vectors to ground displacements measured geodetically. Adapting the PCAIMinversion method in order to accommodate viscoelastic Green's functions is the subject of further study.

\subsection{Results}

Figure 3.6 illustrates viscoelastic responses to coseismic slip for Maxwell rheologies with viscosities of $10^{17}-10^{20} \mathrm{~Pa} \cdot \mathrm{s}$ and for a Burger's body with a short-term transient viscosity of $10^{17} \mathrm{~Pa} \cdot \mathrm{s}$ and steady-state viscosity of $10^{20} \mathrm{~Pa} \cdot \mathrm{s}$. To illustrate the spatial extent and characteristics of the viscoelastic displacement, we illustrate in Figure 3.7 the near-field horizontal and vertical ground displacement and the far-field horizontal ground displacement for the Burger's model.

It is evident from Figure 3.6 and 3.7 that the viscoelastic response to coseismic stress changes does not account for several of the features observed in the cGPS time series. In particular, neither the observed southward motion of sites such as PYGR located within the dashed box in Figure 3.7a nor the spatial and temporal patterns of the observed ground displacement are well fit. Lower values of viscosity, such as $10^{17} \mathrm{~Pa} \cdot \mathrm{s}$, yield rapid decays in the postseismic displacement for sites near the epicentral area, which is consistent with observations of the east component of displacement, but at far-field sites the decay rate of calculated displacement exceeds that observed.

To improve the fit with geodetic observations, we next consider the effects of timedependent afterslip coupled with viscoelastic rebound. Appropriate values for viscosity 

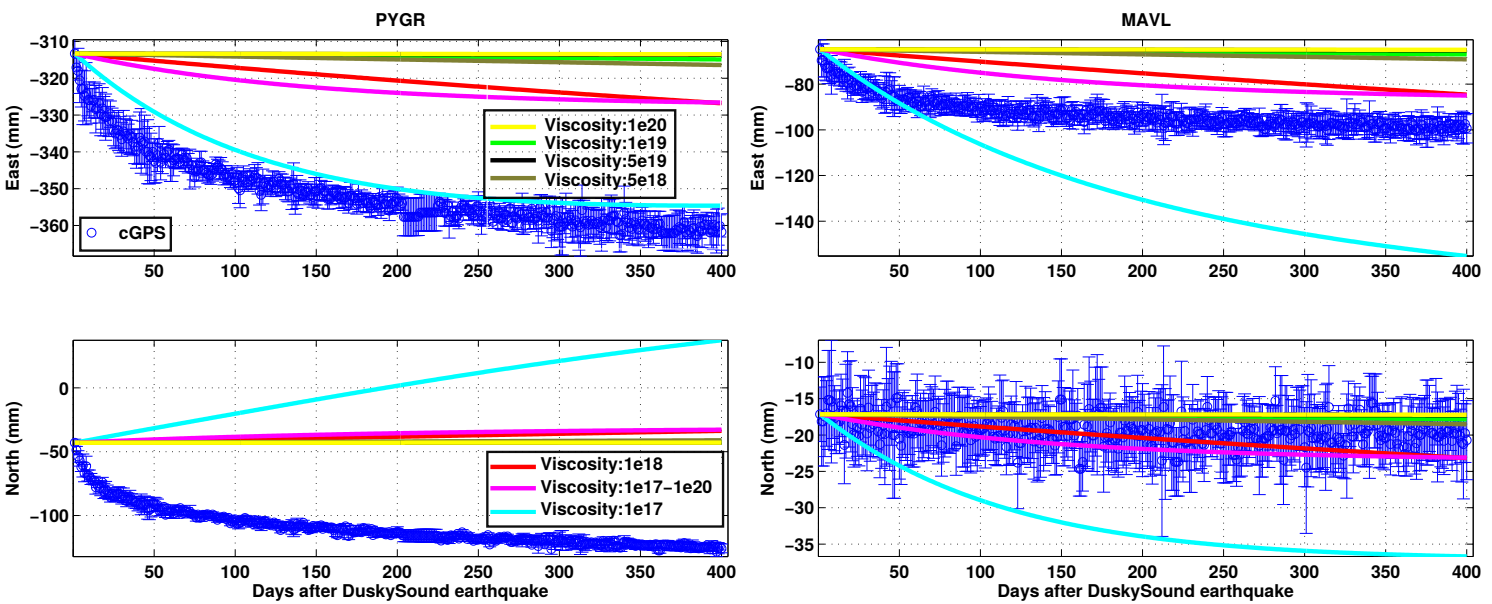

BLUF
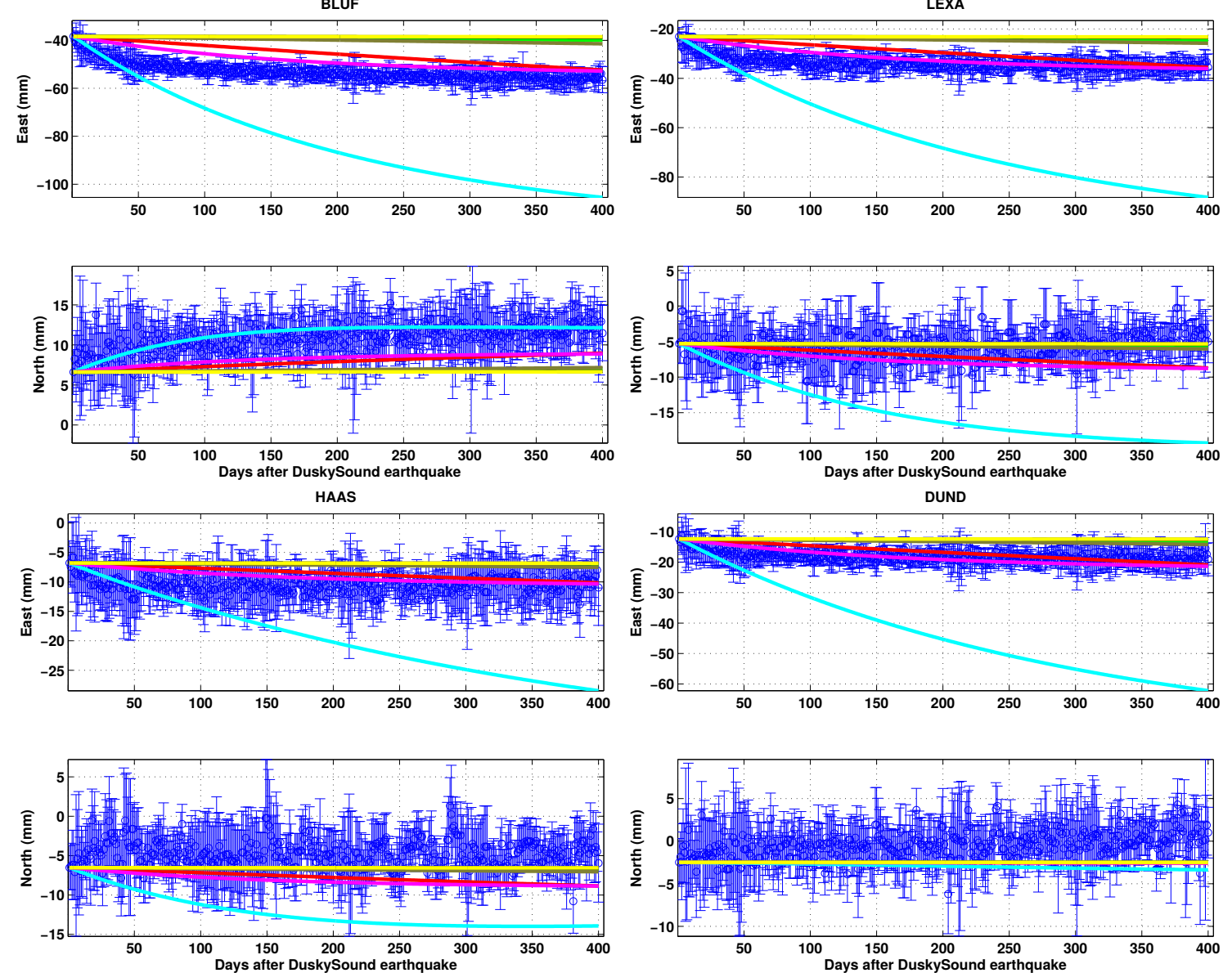

Figure 3.6: cGPS displacement observations (blue symbols) and predicted viscoelastic displacement induced by the earthquake (coloured lines) calculated for a Maxwell rheology of different viscosity values $\left(10^{17}, 10^{18}, 5 \times 10^{18}, 10^{19}, 5 \times 10^{19}\right.$ and $\left.10^{20} \mathrm{~Pa} \cdot \mathrm{s}\right)$ and a Burger's body with transient and steady-state viscosity values of $10^{17}$ and $10^{20} \mathrm{~Pa} \cdot \mathrm{s}$, at PYGR, MAVL, BLUF, LEXA, HAAS, DUND sites.

are chosen by decomposing the displacement time series after subtracting the viscoelastic response to coseismic slip. Figure 3.8 illustrates the first and second temporal modes of the displacement after removing viscoelastic responses to the earthquake induced in Maxwell materials of two different viscosities, $10^{18} \mathrm{~Pa} \cdot \mathrm{s}$ and $5 \times 10^{18} \mathrm{~Pa} \cdot \mathrm{s}$. The first 

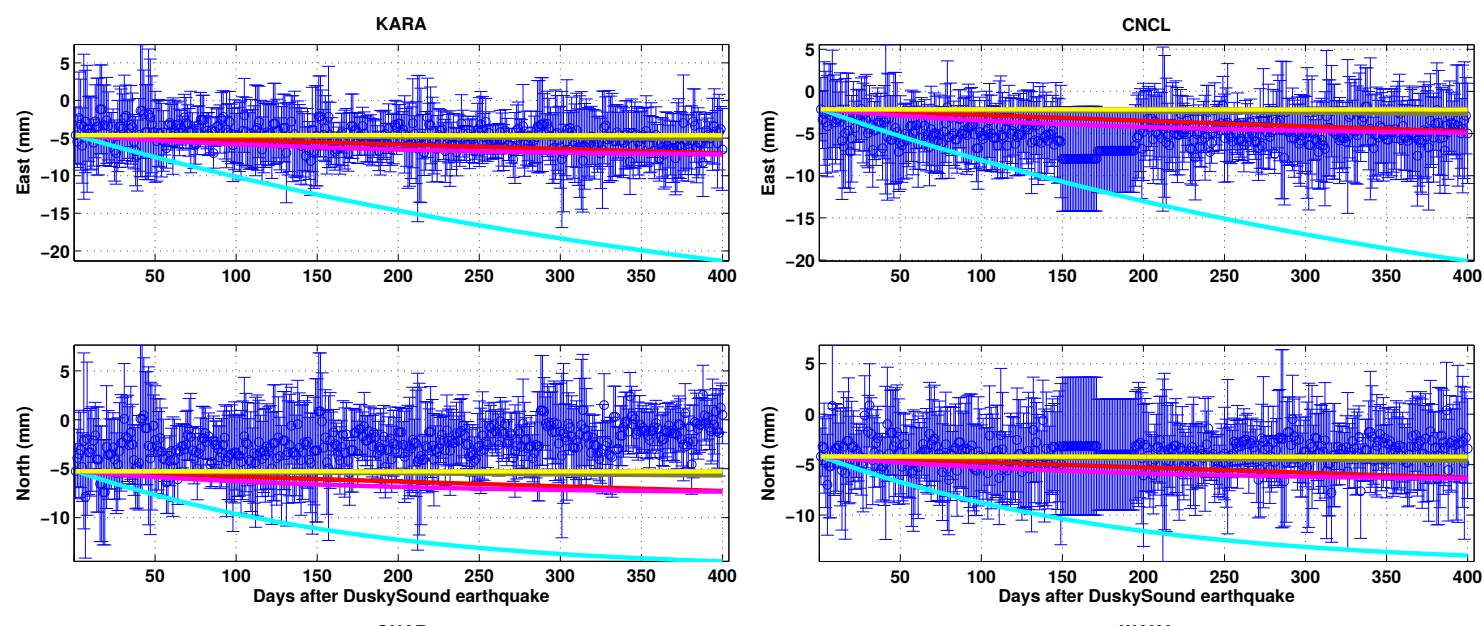

QUAR

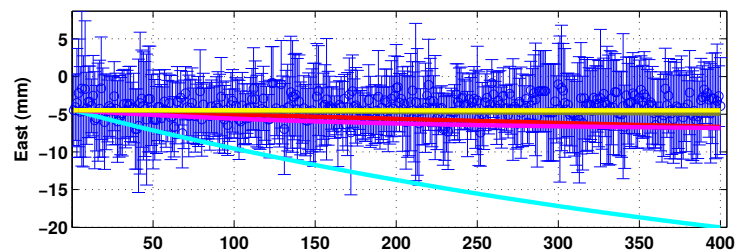

WAKA
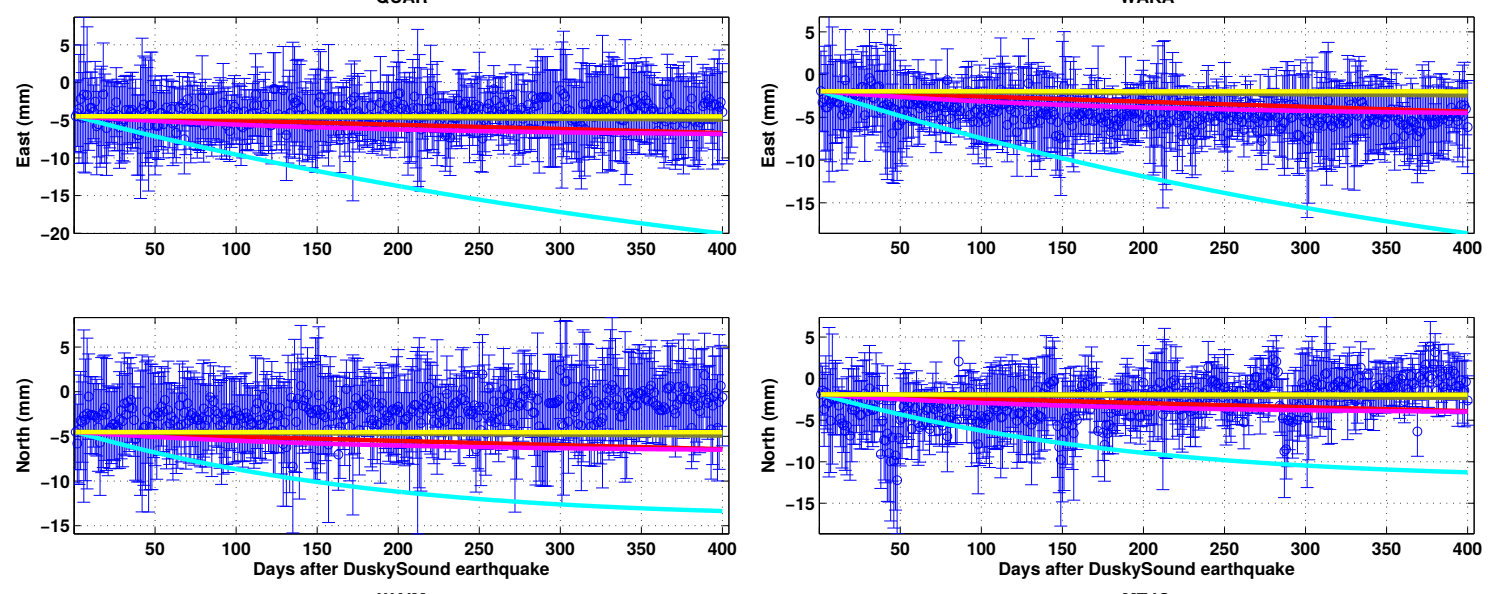

WAIM
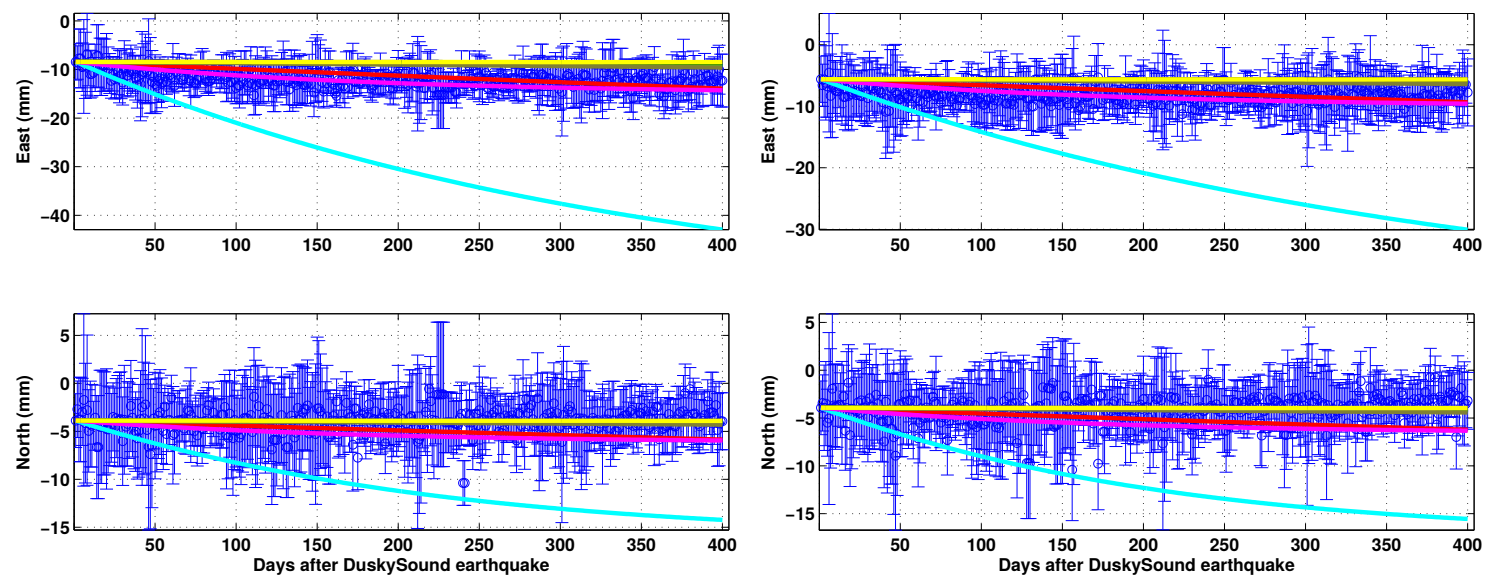

cGPS displacement observations (blue symbols) and predicted viscoelastic displacement induced by the earthquake (coloured lines) calculated for a Maxwell rheology of different viscosity values $\left(10^{17}, 10^{18}, 5 \times 10^{18}, 10^{19}, 5 \times 10^{19}\right.$ and $\left.10^{20} \mathrm{~Pa} \cdot \mathrm{s}\right)$ and a Burger's body with transient and steady-state viscosity values of $10^{17}$ and $10^{20} \mathrm{~Pa} \cdot \mathrm{s}$, at KARA, CNCL, QUAR, WAKA, WAIM and MTJO sites.

temporal modes (i.e. the first column of $V$; V1) shown in blue resemble each other for both viscosities, whereas the second temporal modes V2 shown in red are distinctly different. For a viscosity value of $10^{18} \mathrm{~Pa} \cdot \mathrm{s}, \mathrm{V} 2$ reaches a maximum value almost 100 days after the mainshock; in contrast, for a viscosity value of $5 \times 10^{18} \mathrm{~Pa} \cdot \mathrm{s}$ (illustrated) 

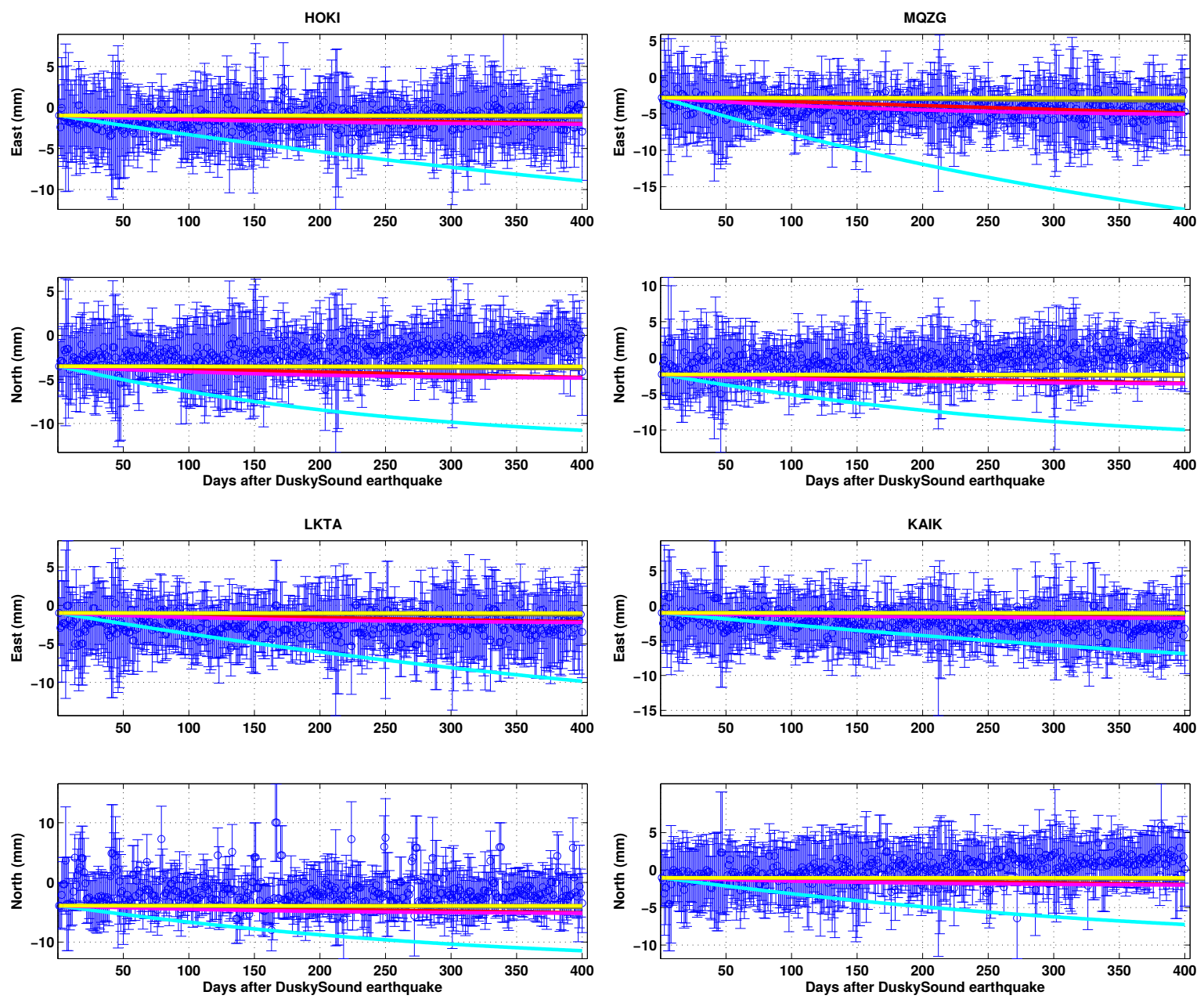

cGPS displacement observations (blue symbols) and predicted viscoelastic displacement induced by the earthquake (coloured lines) calculated for a Maxwell rheology of different viscosity values $\left(10^{17}, 10^{18}, 5 \times 10^{18}, 10^{19}, 5 \times 10^{19}\right.$ and $\left.10^{20} \mathrm{~Pa} \cdot \mathrm{s}\right)$ and a Burger's body with transient and steady-state viscosity values of $10^{17}$ and $10^{20} \mathrm{~Pa} \cdot \mathrm{s}$, at HOKI, MQZG, LKTA and KAIK sites.

or higher, V2 exhibits only random variations.

As discussed in Section 3.2.2, in an elastic medium a slip distribution in time maps directly to the time function of the decomposed ground displacement. Therefore, a temporal mode with a turning point either implies a change of slip direction on the fault, the existence of a different source of displacement in the opposite direction, or under-estimation of viscosity value. We consider a change in the direction of afterslip to be physically unreasonable. If the viscoelastic response to afterslip occurs in the opposite direction resulting in a temporal mode with a turning point, the method described in flowchart 3.5 cannot be used. The reason is that we are limited to perform the inversion on the observational data using elastic Green's functions. Accordingly, based on the direction change in the second temporal mode associated with viscosities equal to or lower than $10^{18} \mathrm{~Pa} \cdot \mathrm{s}$, we adopt viscosities of $5 \times 10^{18}-10^{20} \mathrm{~Pa} \cdot \mathrm{s}$ for the coupled model. 

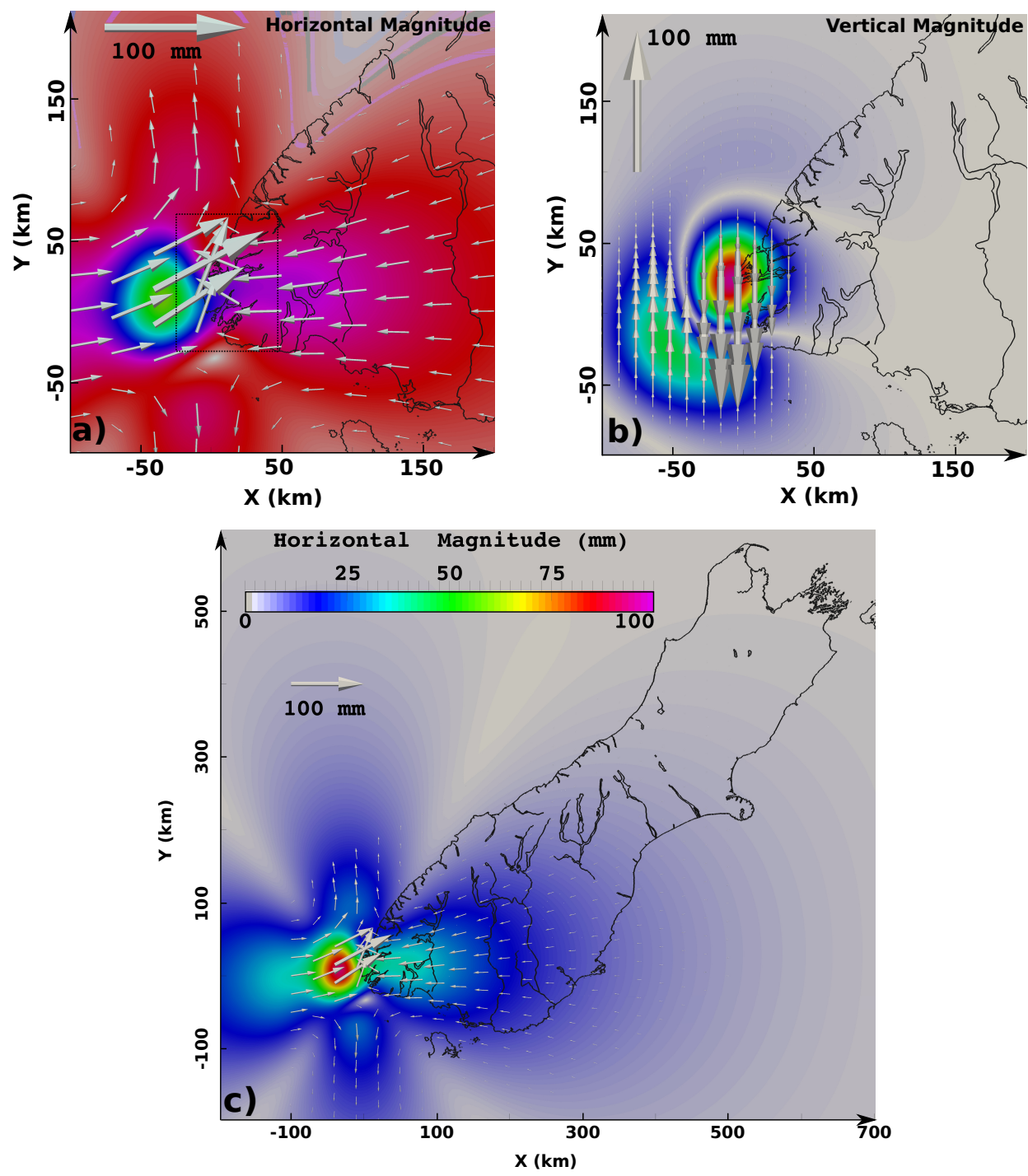

Figure 3.7: a) Horizontal, b) Vertical and c) Regional horizontal components of accumulated displacement due to the viscoelastic rebound of a Burger's body with a transient viscosity of $10^{17}$ and steady-state viscosity $10^{20} \mathrm{~Pa}$.s. Corresponding time series of displacements are shown as magenta in Figure 3.6.

After choosing appropriate values for viscosity, we perform the inversion method. In principle, the iterative calculations must be continued until the modelled ground displacement from the coupled afterslip and viscoelastic displacement match the geodetic observations. We find that only one iteration is required to produce satisfactory fit with GPS and InSAR observations. We can explain that to be the result of insignificant viscoelastic response to time-dependent afterslip with a maximum slip value of $\sim$ $1 \mathrm{~m}$, accumulated within 400 days.

We consider the extension of the coseismic fault surface to be $20 \mathrm{~km}$ on each side and to a maximum depth of $200 \mathrm{~km}$. Throughout the iterative approach, four options have been considered for the boundary of the estimated slip on the extended fault surface, Option 1: unconstrained slip boundary (Figure 3.9a); Option 2: no slip-boundary 

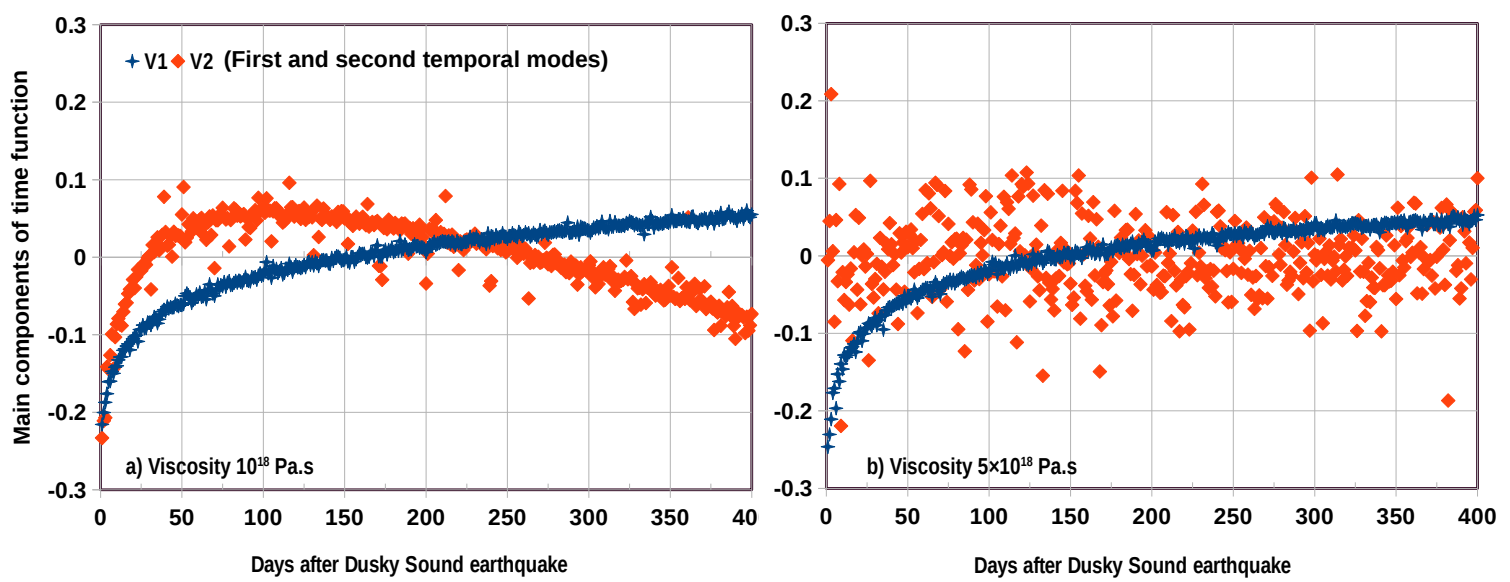

Figure 3.8: First and second temporal modes (V1 and V2) obtained by decomposing the cGPS data after removing the coseismic viscoelastic rebound effects with a viscosity of a) $10^{18} \mathrm{~b}$ ) and $5 \times 10^{18} \mathrm{~Pa} \cdot \mathrm{s}$. For the first viscosity, we can see that the results of SVD method suggest direction change within 400 days. Therefore we only run the models with viscosities higher than $10^{18} \mathrm{~Pa} \cdot \mathrm{s}$.

constraint on the sides, top and bottom of the fault (Figure 3.9b); Option 3: no-slip boundary constraint on the sides of the fault (Figure 3.9c); and Option 4: no-slip boundary constraint on the sides and bottom of the fault (Figure 3.9d). Misfits with cGPS observations are measured in terms of RMSE values. RMSE values of $1.8 \mathrm{~mm}$ are computed for Option 1, Option 3 and Option 4 with a viscosity value of $5 \times 10^{18}$ $\mathrm{Pa} \cdot \mathrm{s}$ and an elastic thickness of $45 \mathrm{~km}$. A RMSE value of $1.9 \mathrm{~mm}$ is obtained from Option 2 with no-slip boundary constraint on the sides, top and bottom of the fault.

The campaign GPS data acquired in August 2009 include the coseismic signal as well as early postseismic effects. consequently, direct measurements of the early postseismic displacement for the campaign GPS sites are not available. We subtract the coseismic signal estimated by Beavan et al. (2010a) from the observed displacement, in order to extract the early postseismic displacement at the campaign GPS sites. Figure 3.10a and b illustrate fits with horizontal and vertical displacements calculated from the postseismic coupled model spanning the time interval reoccupied by the campaign observations (Option 4; observation and modelling results are presented in blue and cyan, respectively). We partially associate the misfit with the residuals of coseismic displacement estimated by Beavan et al. (2010a). The sites with poor accuracy of GPS measurements based on the uncertainty values published by Beavan et al. (2010a) are indicated with grey stars.

We assign uniform weights to the campaign and InSAR measurements. Figure 3.11 illustrates the observed and estimated displacements of the postseismic model for Option 

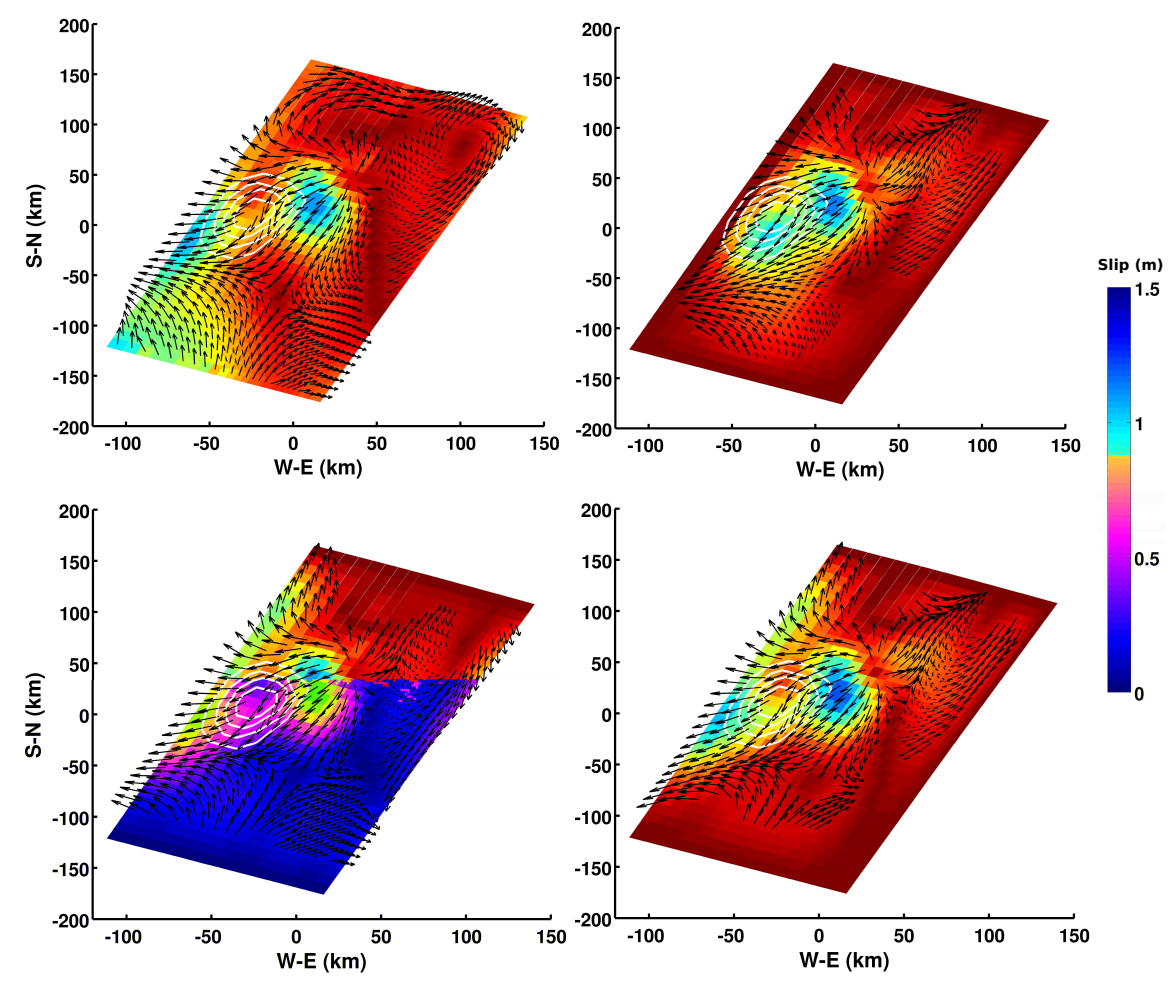

Figure 3.9: Accumulated slip 400 days after the Dusky Sound earthquake. a) Option 1: unconstrained slip boundary. b) Option 2: no slip-boundary constraint on the sides, top and bottom of the fault. c) Option 3: no-slip boundary constraint on the sides of the fault. d) Option 4: no-slip boundary constraint on the sides and the bottom of the fault. Viscosity and the thickness of elastic layer are assumed to be $5 \times 10^{18} \mathrm{~Pa} \cdot \mathrm{s}$ and $45 \mathrm{~km}$, respectively.
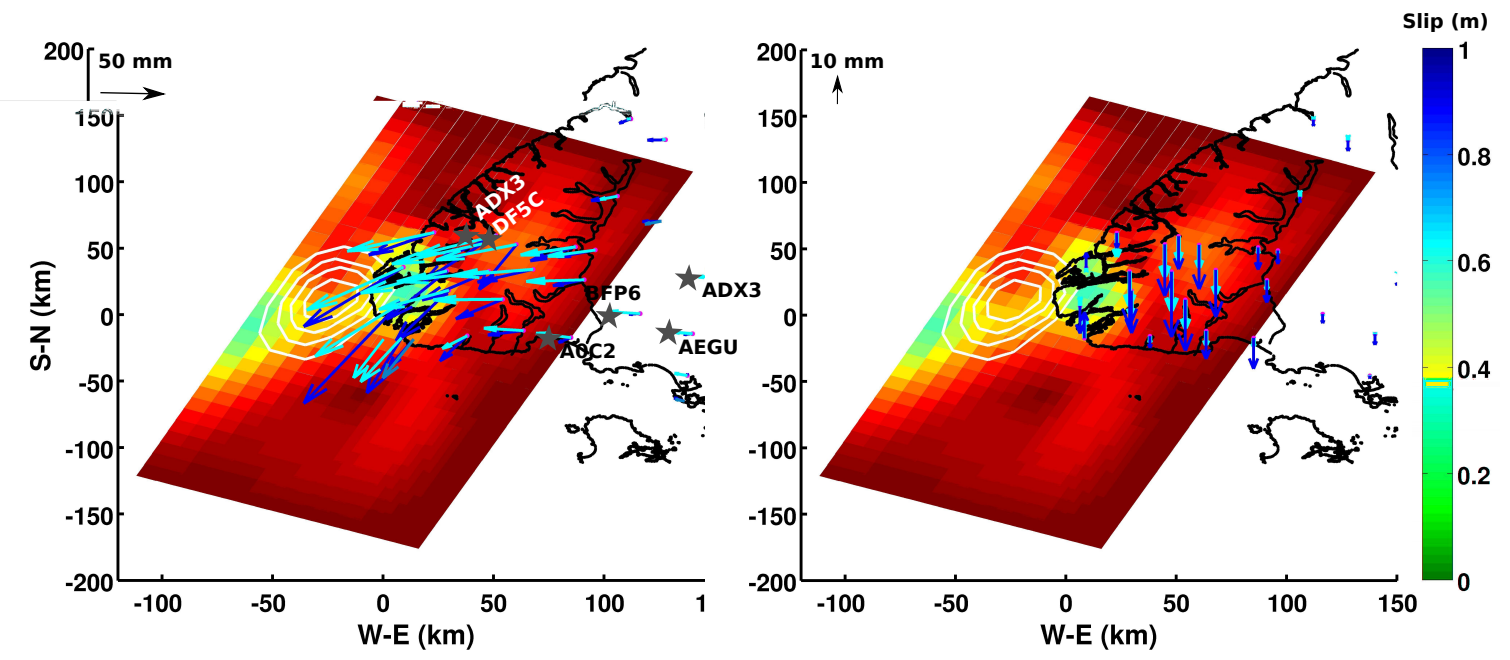

Figure 3.10: a) Horizontal and b) Vertical fits with the early postseismic displacement (August 2009) for the coupled viscoelastic rebound with a viscosity of $5 \times 10^{18} \mathrm{~Pa} \cdot \mathrm{s}$ and time-dependent afterslip (Option 4). The coseismic displacement estimated by Beavan et al. (2010a) is subtracted from the measurements in a-b.

4 in ascending (a, b) and descending ALOS orbits (c, d).

For each of Option 1-4, we identify two main patches located on either sides of the coseismic slip patch (white contours in Figure 3.9) reaching to the ground surface and 

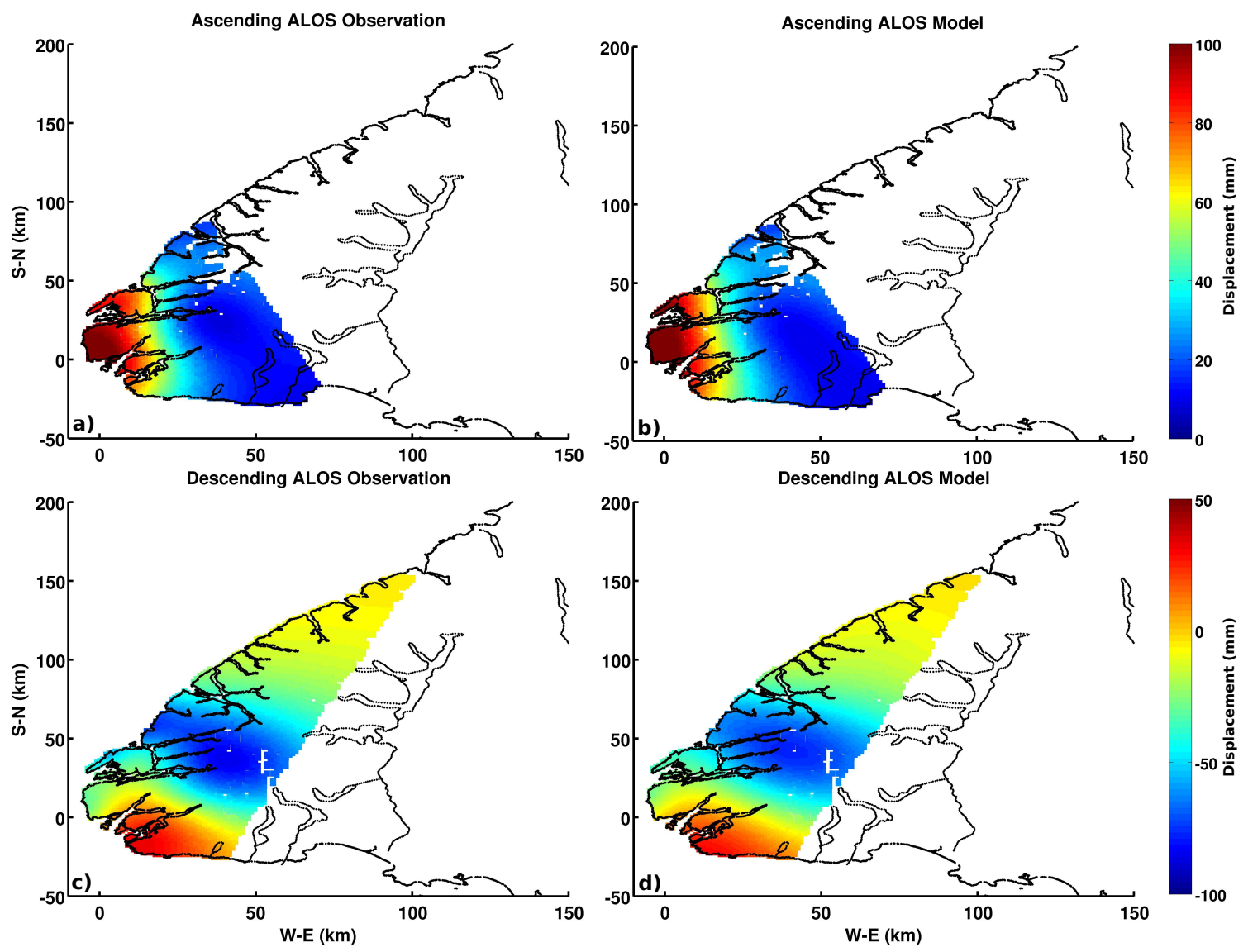

Figure 3.11: a) Observed and b) Estimated postseismic displacements, calculated from Option 4 of the coupled model, projected in the Line Of Sight (LOS) direction of ALOS ascending orbit. c) Observed and d) Estimated postseismic displacements, calculated from Option 4 of the coupled model, projected in the LOS direction of ALOS descending orbit.

depth $80 \mathrm{~km}$. The coupled viscoelastic and afterslip model accounts well for both the spatial and temporal patterns of postseismic displacement with RMSE of $1.8 \mathrm{~mm}$ for Option 4.

Figure 3.12 shows the displacement time series corresponding to Option 4 of the coupled model (red) and observations (blue) at 16 continuous GPS sites. The patterns of ground displacement due to the same coupled model, with a viscosity of $5 \times 10^{18} \mathrm{~Pa} \cdot \mathrm{s}$ and a $45 \mathrm{~km}$ thick elastic layer, for a Maxwell material are illustrated in Figure 3.13. The accumulated far-field horizontal ground displacements, 400 days after the earthquake estimated from the coupled time-dependent afterslip and viscoelastic rebound model are presented in Figure 3.13c. The figure shows larger responses but similar directions compared with the ground displacement calculated from the viscoelastic rebound model of the Burger's body ( Figure 3.13c compared to Figure 3.7c). In the western portion of the dashed box, the purely viscoelastic model predicts north-directed movement (Figure 3.7a), while the coupled model predicts southward movement (Figure 3.13a). Station "PYGR", which is located within this region, displays movement that is more 

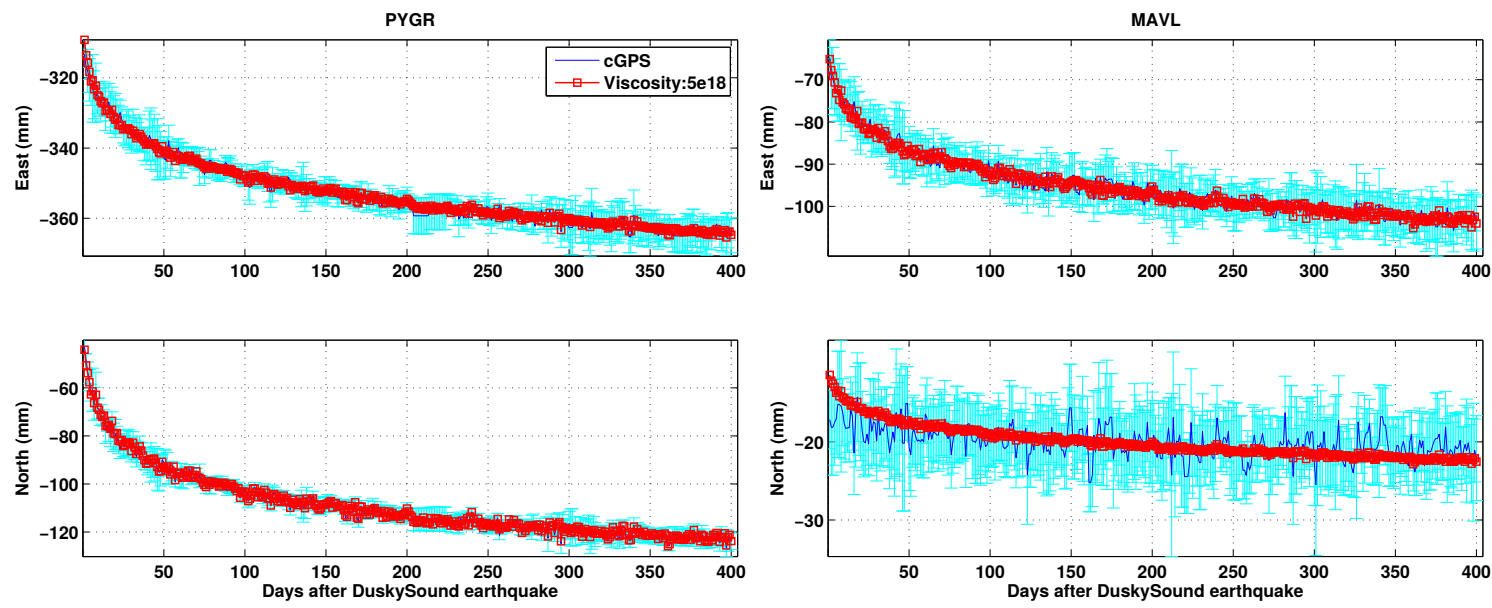

BLUF
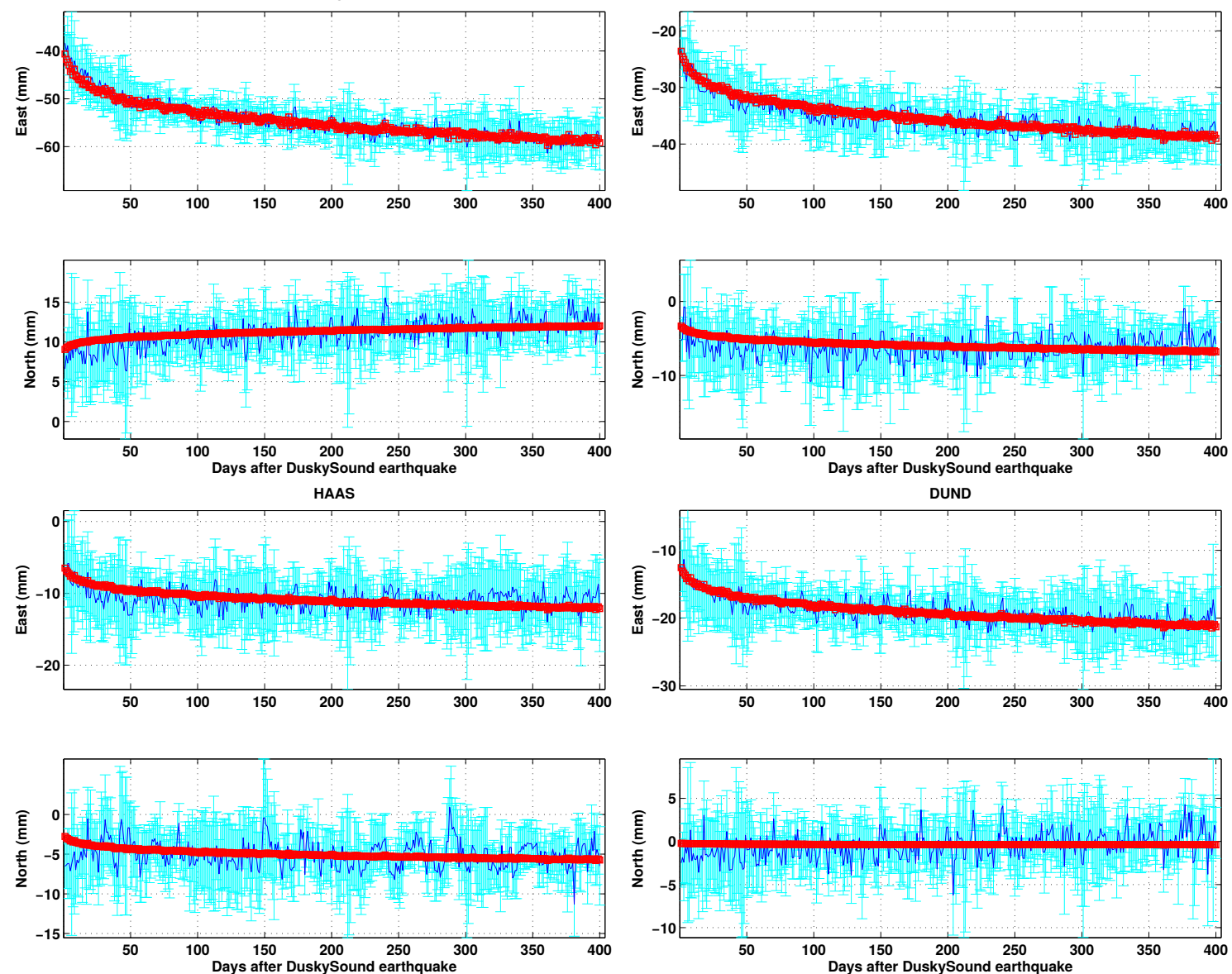

Figure 3.12: cGPS displacement observations (blue symbols) and predicted coupled afterslip with viscoelastic rebound (red lines) calculated for a Maxwell rheology of viscosity $5 \times 10^{18}$ Pa.s, at PYGR, MAVL, BLUF, LEXA, HAAS, DUND sites (Option 4).

consistent with the coupled model. Subsidence patch located in-land from the coast and a partially off-shore uplift patch are the main features of the vertical displacement calculated 400 days after the earthquake from the postseismic model (Figure 3.13b). The vertical component shows an uplift signal at the nearest site "PYGR". 

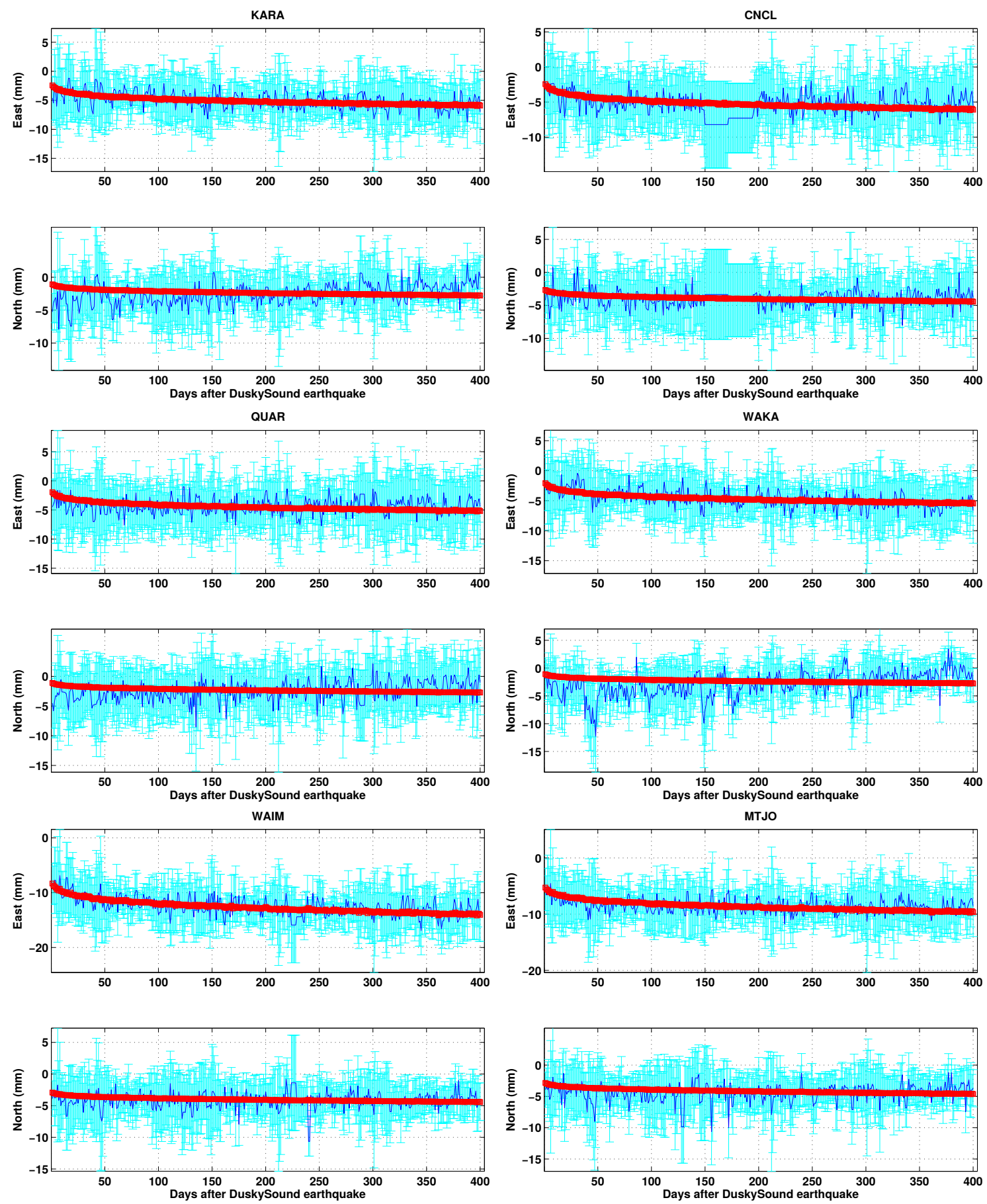

cGPS displacement observations (blue symbols) and predicted coupled afterslip with viscoelastic rebound (red lines) calculated for a Maxwell rheology of viscosity $5 \times 10^{18} \mathrm{~Pa} \cdot \mathrm{s}$, at KARA, CNCL, QUAR, WAKA, WAIM and MTJO sites (Option 4). 

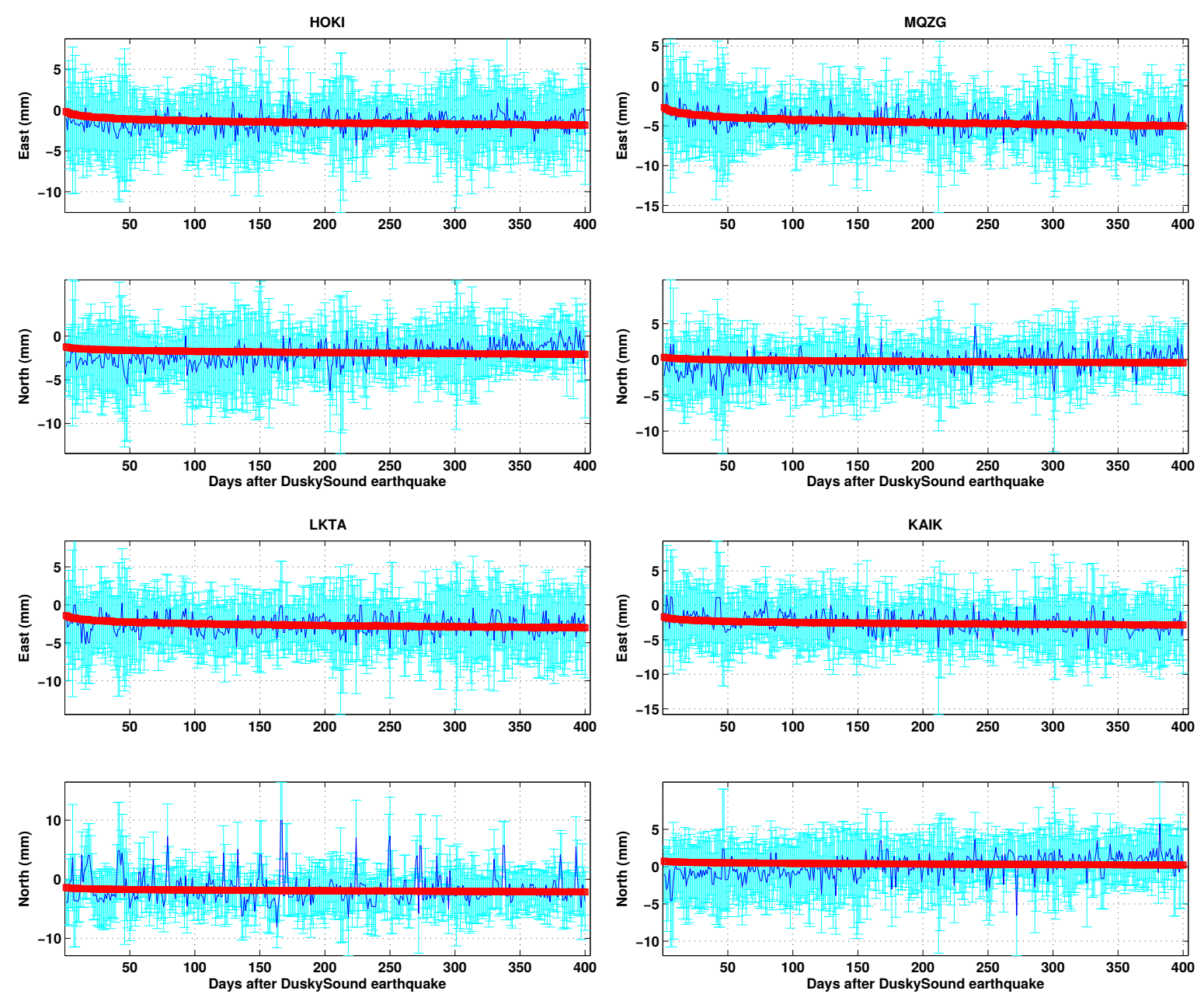

cGPS displacement observations (blue symbols) and predicted coupled afterslip with viscoelastic rebound (red lines) calculated for a Maxwell rheology of viscosity $5 \times 10^{18} \mathrm{~Pa} \cdot \mathrm{s}$, at HOKI, MQZG, LKTA and KAIK sites (Option 4). 

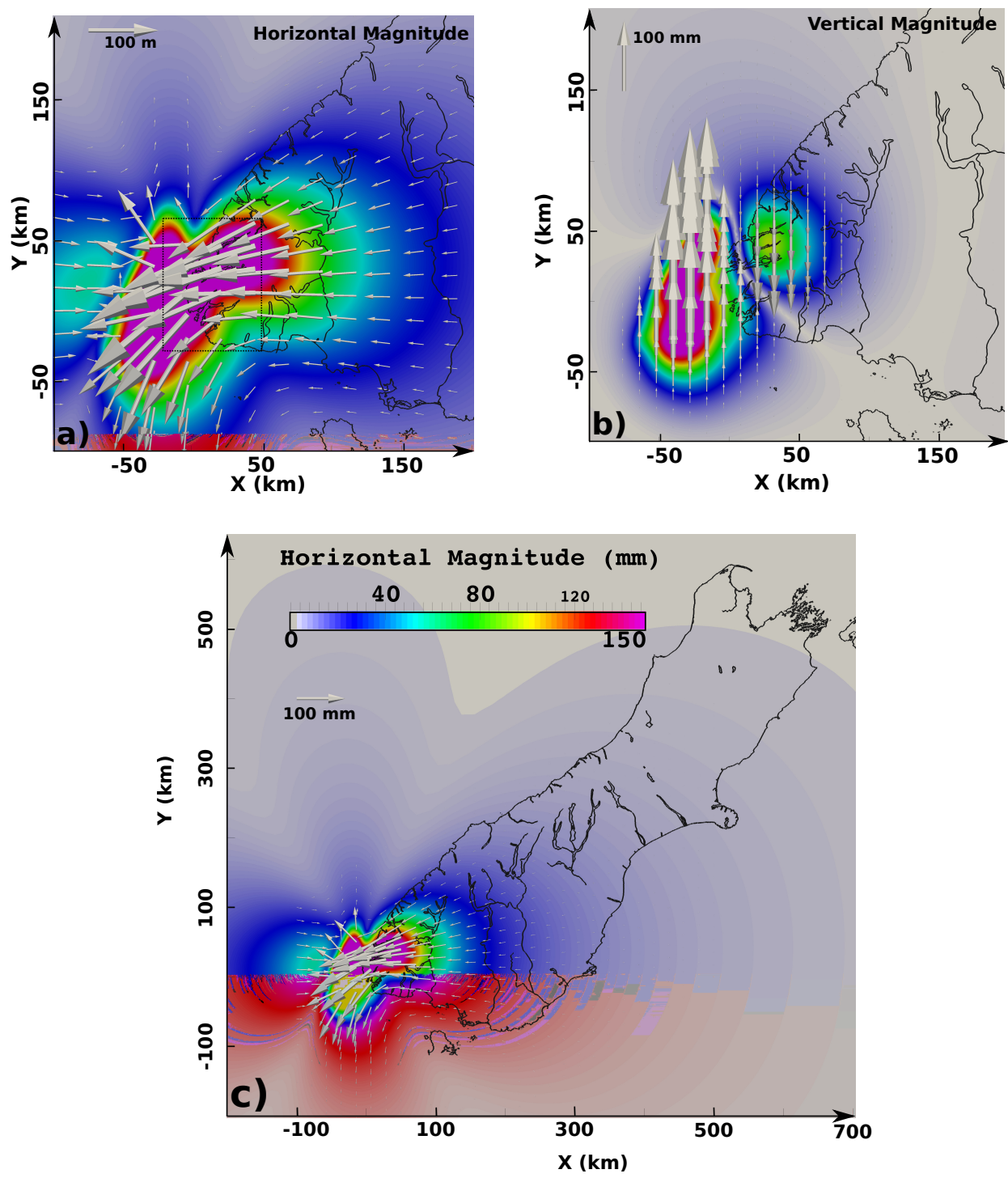

Figure 3.13: a) Horizontal, b) Vertical and c) Regional horizontal components of accumulated displacement due to coupled viscoelastic rebound (viscosity $5 \times 10^{18} \mathrm{~Pa} \cdot \mathrm{s}$ ) and time-dependent afterslip 400 days after Dusky Sound earthquake (Option 4). Corresponding time series of displacements are shown as red in Figure 3.12.

\subsection{Discussion}

\subsubsection{Alternative viscoelastic models}

We attempt to elucidate the postseismic signal following the Dusky Sound earthquake by examining viscoelastic responses to the earthquake for different rheologies. The examined values of viscosity, range from $10^{17}$ to $10^{20} \mathrm{~Pa} \cdot \mathrm{s}$, for the lower crust and the mantle in this chapter are consistent with values assumed elsewhere, from very low ( $<=10^{17} \mathrm{~Pa} \cdot \mathrm{s}$; e.g. Vladimirova et al., 2011; Pollitz et al., 1998a; Ryder et al., 2010a) to very high viscosity $\left(>=10^{20} \mathrm{~Pa} \cdot \mathrm{s}\right.$; e.g. Vladimirova et al., 2011; Pollitz et al., 1998a; 
Table 3.1: Root Mean Square Errors of viscoelastic models for Maxwell rheologies with viscosity values of $10^{17}, 10^{18}, 5 \times 10^{18}, 10^{19}, 5 \times 19$ and $10^{20}$ and a Burger's body with transient and steady-state viscosity values of $10^{17}$ and $10^{20} \mathrm{~Pa} \cdot \mathrm{s}$ at continuous GPS sites.

\begin{tabular}{|c|ccccccc|}
\hline & \multicolumn{7}{|c|}{ No afterslip } \\
\hline Viscosity & $10^{17}$ & $10^{17}-10^{20}$ & $10^{18}$ & $5 \times 10^{18}$ & $10^{19}$ & $5 \times 10^{19}$ & $10^{20}$ \\
\hline RMSE & 25.0 & 14.7 & 14.8 & 15.2 & 15.3 & 15.4 & 15.4 \\
\hline
\end{tabular}

Ryder et al., 2010a).

The nearest site to the epicentral area is PYGR, which exhibited westward and southward postseismic motion. The computed viscoelastic response involves westward and northward motion; in other words, we are unable to achieve reasonable fit with the observed north component of the measured displacement. Neither varying the Maxwell viscosity from $10^{17}$ to $10^{20} \mathrm{~Pa} \cdot \mathrm{s}$ nor altering the Burger's transient viscosity of $10^{17}$ to steady-state viscosity of $10^{20} \mathrm{~Pa} \cdot \mathrm{s}$ can account for the southward displacement at PYGR (Figure 3.6).

The viscoelastic response for a very low viscosity of $10^{17} \mathrm{~Pa} \cdot \mathrm{s}$ lags behind the observed east component at the same site, PYGR. Further from the epicentral area the computed viscoelastic response decays more rapidly than the observed east and north components. Accordingly, we conclude that the observed signal cannot be accounted for in terms of viscoelastic rebound only with the range of viscosities from $10^{17}$ to $10^{20} \mathrm{~Pa} \cdot \mathrm{s}$. RMSEs are calculated for all viscoelastic models in Table 3.1. The two timescales obtained by a Burger's body allow us to represent or approximate transient processes (e.g., fault slip) that are not possible with a single viscosity. Consequently, we can see the bi-viscous Burger's body provides better fit with observations compared to other viscoelastic models (RMSE $=14.7 \mathrm{~mm}$ ).

To summarise, the key findings of alternative viscoelastic models neglecting afterslip are as follows:

1. None of the viscoelastic models accounts for the southward motion observed in the epicentral area;

2. The response of a bi-viscous (Burger's) body predicts the observations more accurately than that of a simple Maxwell rheology with different viscosity values (RMSE error $14.7 \mathrm{~mm}$ ); and

3. The simple Maxwell rheology with the lowest viscosity of $10^{17} \mathrm{~Pa} \cdot \mathrm{s}$ deviates significantly from the cGPS measurements with the RMSE of $\sim 25.0 \mathrm{~mm}$. 


\subsubsection{Coupled viscoelastic rebound and afterslip}

The iterative approach for coupling the viscoelastic rebound and time-dependent inversion after achieving reasonable fit with campaign and continuous GPS has been followed. As we described in Section 3.3, using Options 1-4, different boundary constraints have been imposed within the PCAIM-inversion method. The main patches using Options 1-4 are located on either sides of the coseismic patch. The unconstrained slip boundary in Option 1 estimates spurious slip on the edges of the extended fault surface. The estimated slip on the off-shore southern side of the fault surface is not well constrained with observations due to the lack of GPS and InSAR observations in the region. We also infer non-uniform rake angles, inconsistent with the average coseismic rake by Beavan et al. (2010a) and the expected rake of the plate motion on the plate interface (DeMets et al., 2010; Beavan et al., 2002), on the edges of the fault surface. Option 2 with a RMSE value of $1.9 \mathrm{~mm}$ presented a relatively poor estimation of the postseismic displacement model. The poor accuracy of Option 2 indicates that slip reaches the top edge of the fault surface. Although slip is allowed on the bottom edge of the fault surface, Option 3 predicts insignificant slip at the bottom extension of the fault surface. Thus Option 4 is selected as the final preferred postseismic slip model in an elastic-viscoelastic medium with viscosities of $5 \times 10^{18}-10^{20} \mathrm{~Pa} \cdot \mathrm{s}$.

The grey stars in Figure 3.10 represent sites with inaccurate postseismic measurements. We suspect the higher uncertainties at these sites stem from uncertainties in the estimation of secular motion and removal from the total displacement. At these locations, the misfit of the model with campaign measurements, in spite of satisfactory fits to the cGPS data is explained as a combination of

1. Uncertainties in the coseismic model used to remove the coseismic part from the total displacement. Early postseismic measurements are contaminated by coseismic deformation.

2. Uncertainties in the estimation of secular motion at several sites.

\subsubsection{Stress and strain changes in Canterbury}

We can address the question of whether or not the Canterbury earthquake sequence of 2010-2012 was advanced by the Dusky Sound earthquake of 2009 by better understanding the mechanisms of postseismic displacement. We consider what the cumulative stress changes produced by the Dusky Sound earthquake were at the hypocentre 
of the Darfield earthquake, which originated on the reverse Charing Cross Fault, and at the centre of the Greendale Central Fault on which most slip subsequently occurred (Beavan et al., 2010b).

The geometry and the average rake angle of the reverse Charing Cross Fault and the right-lateral Greendale Central Fault were determined by Beavan et al. (2012b). We calculate the cumulative Coulomb stress changes produced by coseismic and postseismic displacement 400 days after the Dusky Sound earthquake on each of these faults at a depth of $4 \mathrm{~km}$. We assume an isotropic poroelastic rheology for the frictional model (Harris, 1998) with Skempton's and frictional coefficients of 0.5 and 0.6, respectively.

On the Charing Cross Fault, striking $035^{\circ}$ and dipping $070^{\circ}$ south-east, the Coulomb stress changes for both postseismic (Figure 3.14a) and accumulated coseismic and postseismic processes (Figure 3.14b) are negative. In contrast, on the Greendale Central Fault, which strikes $086^{\circ}$ and dips $080^{\circ}$ southwards, the Coulomb stress changes induced by postseismic mechanisms alone (Figure 3.14c) or by coseismic and postseismic mechanisms together (Figure 3.14d) after 400 days show positive changes of the order of a few kilopascals.

Our analysis indicates that the Coulomb stress changes due to the Dusky Sound earthquake are consistent with further ruptures on the Greendale Fault in the central South Island, in the Canterbury region but of a very small order. However, the Darfield rupture initiated on the Charing Cross Fault (Holden et al., 2011; Gledhill et al., 2010, 2011) on which negative Coulomb stress changes are estimated due to the Dusky Sound earthquake.

Another way of considering the effects of the Dusky Sound earthquake on the Canterbury region is to examine the pattern of cumulative horizontal strain (Figure 3.15). The cumulative postseismic principal contractional strain and azimuth of maximum horizontal contraction can be calculated from cGPS observations and compared with those inferred from the numerical model. Monte Carlo bootstrapping is used to estimate uncertainties in the strain parameters. Figure 3.15 illustrates the strain ellipses for three different areas in the southern South Island. The ellipses are annotated with the $95 \%$ confidence intervals for the principal strains and axes. The results shown in blue are computed from data recorded at all 16 cGPS sites in Figure 4.6. The results and corresponding stations illustrated in green and red represent smaller areas.

Using all 16 cGPS sites yields spatially averaged estimates for the cumulative principal strain components and horizontal contraction azimuth, with values of [8.3 to 13$] \times 10^{-8}$ 

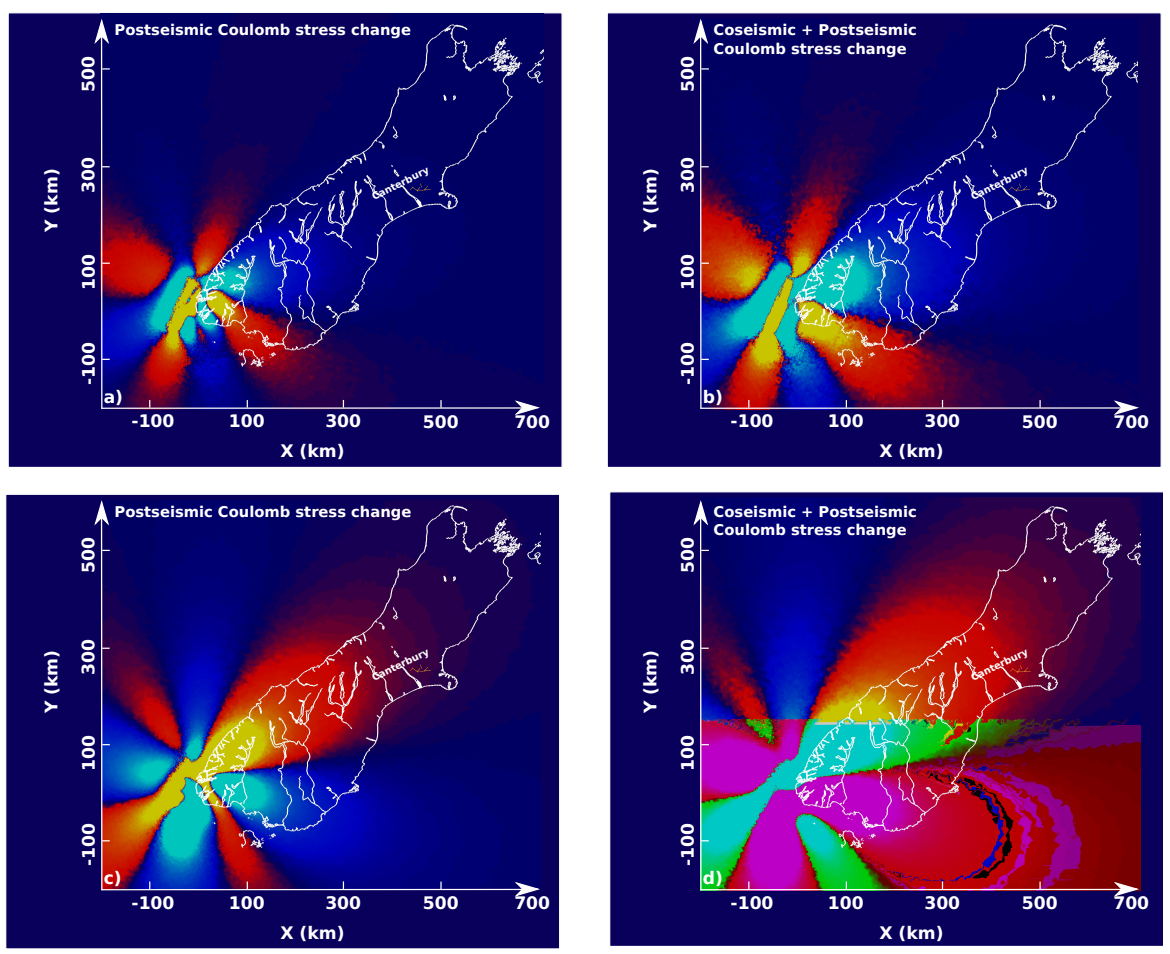

Figure 3.14: a) The receiver fault is set to the geometry of the Charing Cross Fault in order to calculate the cumulative Coulomb stress changes induced by the coupled viscoelastic rebound with a viscosity of $5 \times 10^{18} \mathrm{~Pa} \cdot \mathrm{s}$ and time-dependent afterslip, b) The receiver fault is set to the geometry of the Charing Cross Fault in order to calculate the cumulative Coulomb stress changes induced by the coupled model added to the coseismic stress changes, c) The receiver fault is set to the geometry of the Greendale Fault in order to calculate the cumulative Coulomb stress changes induced by the coupled model and d) The receiver fault is set to the geometry of the Greendale Fault in order to calculate the cumulative Coulomb stress changes induced by the coupled model added to the coseismic stress changes. The isotropic poroelastic rheology for the frictional model is assumed with Skempton's and frictional coefficients of 0.5 and 0.6, respectively. For Greendale Fault, strike $086^{\circ}$ and dip $080^{\circ}$ southward, for Charing Cross Fault, strike $035^{\circ}$ and dip $070^{\circ}$ south-east are considered. All calculations are performed within 400 days after the Dusky Sound earthquake at a depth of $4 \mathrm{~km}$.

$\left(\right.$ mean $\left.9.4 \times 10^{-8}\right),[-8.6$ to -0.5$] \times 10^{-8}\left(\right.$ mean $\left.-3.0 \times 10^{-8}\right)$ and $\left[111^{\circ}\right.$ to $\left.157^{\circ}\right]($ mean $\left.143^{\circ}\right)$, respectively, 400 days after the Dusky Sound earthquake. The corresponding values for the green and red subsets are $[-18.1$ to 21.9$] \times 10^{-8}\left(\right.$ mean $\left.15.4 \times 10^{-8}\right)$, $[-14.2$ to +11.2$] \times 10^{-8}\left(\right.$ mean $\left.-4.4 \times 10^{-8}\right)$ and $\left[110^{\circ}\right.$ to $\left.167^{\circ}\right]\left(\right.$ mean $\left.144^{\circ}\right)($ green, southern $)$ and $[-1.2$ to 1.8$] \times 10^{-8}\left(\right.$ mean $\left.1.2 \times 10^{-8}\right),[-1.0$ to +0.9$] \times 10^{-8}($ mean $-0.4 \times$ $10^{-8}$ ) and $\left[79^{\circ}\right.$ to $\left.125^{\circ}\right]$ (mean $106^{\circ}$; red, northern; extension is positive and contraction is negative). Other calculations, not shown, demonstrate that a displacement gradient across the South Island from north-west to south-east contributes to the cGPS strain estimates. The red strain set includes more sites spanning the north-west to south-east direction as shown in Figure 3.15, and the effect of the displacement gradient in this direction is to rotate the red strain ellipse anticlockwise with respect to the green one. 


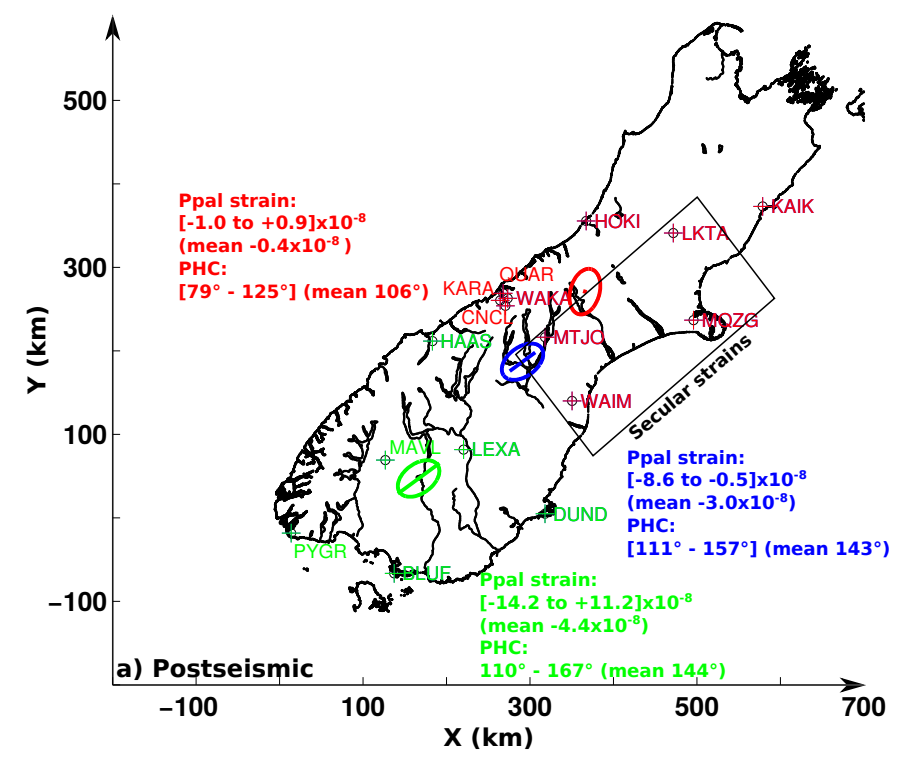

Figure 3.15: Ellipses for strain accumulated 400 days after the Dusky Sound earthquake shown for three strain sets. Blue indicates the strain ellipse for all 16 cGPS observations shown in Figure 4.6. Second and third strain sets and the calculated stain ellipses are illustrated in green and red. The smaller the number of cGPS sites used, the larger the uncertainties that are estimated from Monte Carlo bootstrapping. PHC: Principal Horizontal Contraction azimuth.

Figure 3.16 illustrates the strains calculated using the numerical model of postseismic displacement following the Dusky Sound earthquake, taking into account the coseismic displacement. Maximum extension directions in blue and contraction directions in red are plotted on a background of the second invariant and, for clarity, we only show results in the south-eastern South Island and Canterbury. The upper panel (Figure 3.16a) shows the coseismic strain and the lower panel (Figure 3.16b) shows the sum of coseismic and postseismic strains over a 400 day period.

The postseismic model does not incorporate secular strain in the South Island or any other sources of strain associated, for instance, with the Alpine Fault or other components of the plate boundary. Therefore, the results are not directly comparable with the strains illustrated in Figure 3.15. Nevertheless, some conclusions can be drawn about the observed and modelled distributions of strain. Focusing on the Canterbury region, we note that the modelled direction of maximum contraction is $\sim 151^{\circ}$ similar to the blue and green cGPS estimates (mean $144^{\circ}$ ) but approximately $45^{\circ}$ clockwise around the red cGPS result for both coseismic and the sum of coseismic and postseismic strains. Principal strains $\sim 2.4 \times 10^{-8}$ and $\sim-0.3 \times 10^{-8}$ are calculated near the Darfield earthquake epicentre from the coupled time-dependent model within 400 days of the Dusky Sound earthquake, taking into account the coseismic strain. Principal strains of $-6.9 \times 10^{-8}$ and $2.7 \times 10^{-8}$ and principal horizontal contraction azimuth, $109^{\circ}$ are obtained from the average regional strain rate tensor (annual rate). We calculate 

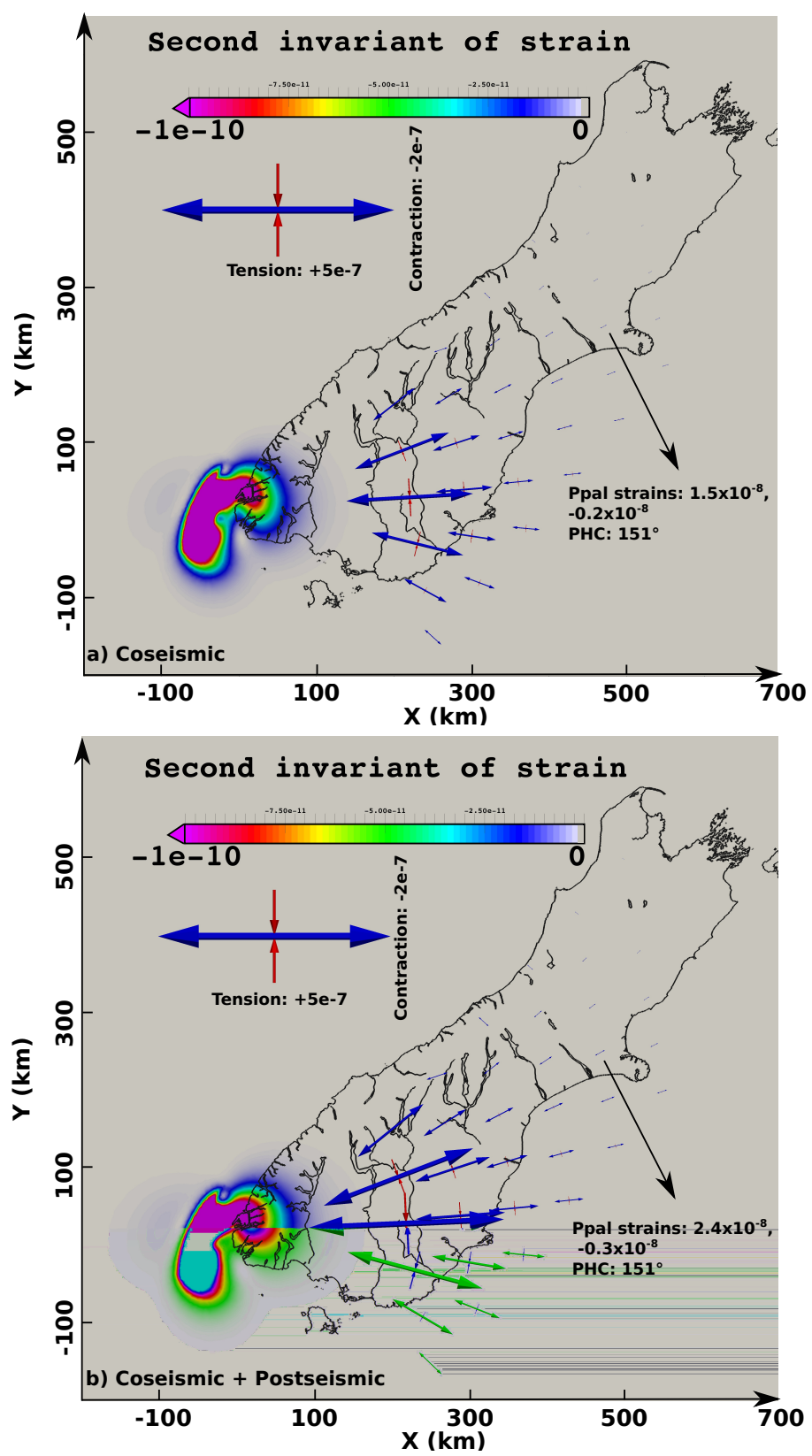

Figure 3.16: a) Coseismic b) The sum of coseismic and cumulative postseismic principal strain components, blue arrows pointing outwards for extension and red arrows pointing inwards for contraction and the color contours show the second invariant of strain (Option $4)$.

the average regional strain rate tensor from the secular velocities of four sites in the box shown in Figure 3.16. The sum of coseismic and postseismic principal extensional and contractional strain rates are approximately $80 \%$ and $4 \%$ of the equivalent secular rates in the Canterbury region, respectively. However, the principal horizontal contraction azimuth of the sum of coseismic and postseismic strain model is oriented $\sim 42^{\circ}$ clockwise from the secular motion contraction axis azimuth. Therefore, even if other sources of strain associated, for instance, with the Alpine Fault or other components of the plate boundary can rotate the modelled contraction axis favourably towards 
$S_{H \max }$, of $116^{\circ} \pm 18$ in the Canterbury region (Holt et al., 2013; Townend et al., 2012), the effect of the Dusky Sound earthquake and the associated postseismic can be only $4 \%$ of the secular motion.

\subsection{Conclusions}

In this study, we examine different postseismic processes following the Dusky Sound earthquake and consider the farfield effects of this event on the subsequent Darfield earthquake. The iterative method described here reveals: 1) afterslip occurring at depths between $0-80 \mathrm{~km}$ on both sides of the coseismic slip patch evolving logarithmically in time; and 2) a viscosity higher than $10^{18} \mathrm{~Pa} \cdot \mathrm{s}$ for the viscoelastic half-space underlying the elastic layer with a thickness of $45 \mathrm{~km}$.

In order to investigate the effect of the Dusky sound earthquake postseismic responses, we compute Coulomb stress and strain models for both postseismic and the sum of postseismic and coseismic changes. Lateral expansion of the region of positive coseismic Coulomb stresses due to the postseismic mechanisms, following the Dusky Sound earthquake on the Greendale Fault, is inferred from Figure 3.14b and c. In addition, contractional and extensional strain arrows (inferred from Figure 3.16a to b) become larger with considering the postseismic strain model. Therefore we infer that the Dusky Sound earthquake postseismic responses enhanced the coseismic Coulomb stress and strain models significantly. However, the earthquake and the associated postseismic mechanisms do not seem to have directly triggered the Darfield earthquake, based on 1) the negative Coulomb stress changes computed for the Charing Cross Fault on which the Darfield earthquake nucleated; 2) positive but very small Coulomb stress changes on the Greendale Fault; and 3) the magnitude of the principal strain rates, $4 \%$ of the regional principal contractional strain rate, and contraction azimuth, $42^{\circ}$ clockwise around the regional contraction axis. 



\section{Chapter 4}

\section{Coupling time-dependent afterslip with viscoelastic relaxation using analytical and numerical Green's functions}

\section{Abstract}

Postseismic displacement following large earthquakes is often described in terms of either time-varying afterslip on the coseismically ruptured surface or its extension, or viscoelastic relaxation. Here, we describe an inversion framework with which to estimate afterslip while taking account of viscoelastic effects. Estimating time-dependent afterslip is an ill-posed problem unless steps are taken to avoid high-frequency components of displacement affecting the calculated slip, and this requires regularisation in both space and time. Conventionally, the problem of computing the slip distribution in space is regularised using Laplacian smoothing of the fault slip. In order to regularise the ill-posed problem in time, we adapt the method of Kositsky and Avouac (2010) to accommodate viscoelastic media. Ground displacement in response to a dislocation source is a linear function of slip for an elastic medium, meaning that Singular Value Decomposition (SVD) of the observed displacement time series yields the temporal evolution of slip. The problem remains linear for a linear viscoelastic medium but in this case the Green's functions are time-dependent. We estimate Green's functions representing the response to both afterslip and viscoelastic relaxation using the finite element code PyLith or the semi-analytical solution of Fukahata and Matsu'ura (2006). With this approach, we decompose the time-dependent Green's functions and the ground displacement into space and time functions and use these to represent the evolution of slip. We apply this method to the postseismic ground displacement observed following the $\mathrm{M}_{\mathrm{W}} 7.8$ Dusky Sound (New Zealand) earthquake of 15 July 2009 and to two numerical and analytical synthetic examples. 


\subsection{Introduction}

Afterslip in the upper/lower crust on the mainshock fault surface (e.g. Savage and Church, 1974; Johnson et al., 2012; Wen et al., 2012a; Reddy et al., 2013) and viscoelastic relaxation in the lower crust/upper mantle (e.g. Elsasser, 1967; Savage and Prescott, 1978; Pollitz et al., 2000; Pollitz, 2003b; Rousset et al., 2012b) are inferred to be the two most predominant postseismic mechanisms following the subduction earthquakes.

Afterslip models often explain the transient displacement following large earthquakes without taking viscoelastic effects into consideration (e.g. Hsu et al., 2002; Miyazaki et al., 2004; Hsu et al., 2006). Time-dependent inversion of continuous global positioning system (GPS) observations yields estimates of the transient afterslip associated with postseismic periods. Such inversion techniques often approximate the slip history in an elastic medium with its corresponding spatial and temporal basis functions and use additional hyperparameters to regularise the problem in both space and time (Segall and Matthews, 1997; Fukuda et al., 2004, 2008).

Several studies have demonstrated that viscoelastic responses are required to match GPS time series data in addition to afterslip (e.g. Azúa et al., 2002; Sheu and Shieh, 2004; Chan and Stein, 2009; Johnson et al., 2009; Bruhat et al., 2011; Diao et al., 2011; Gunawan et al., 2014; Rollins et al., 2015; Sun and Wang, 2015). If the viscoelastic effects of mantle flow are not taken into consideration, very long-term afterslip is required to explain persistent postseismic displacements (e.g. Sawai et al., 2004). However, such prolonged afterslip is incompatible with the characteristic afterslip time-scales observed, which are typically of the order of a few months to a few years (Pritchard and Simons, 2006; Wang et al., 2012). Furthermore, short-term rapid decay of postseismic displacement is often explained by very low values of viscosity and correspondingly short relaxation times (e.g. Palano et al., 2009). Very short relaxation times can also be interpreted in terms of significant afterslip. Therefore, investigating the relative contributions of afterslip and viscoelastic flow in the mantle is essential.

A forward frictional afterslip model in its rate-strengthening form (e.g. Ruina, 1983; Dieterich, 1994; Lapusta et al., 2000; Rice et al., 2001; Marone et al., 1991; Johnson et al., 2006; Barbot et al., 2009; Barbot and Fialko, 2010a) and corresponding viscoelastic responses form a unified framework that incorporates coupled processes of afterslip and viscoelastic flow (e.g. Perfettini et al., 2005; Hearn et al., 2009; Barbot et al., 2009; Johnson et al., 2009; Barbot and Fialko, 2010a). The rate-strengthening form of the friction theory restricts afterslip to locations outside the coseismic patch at 
which stresses increase coseismically (Barbot et al., 2009; Johnson et al., 2009). The other method of estimating afterslip is to invert geodetic observations in which the necessary slip occurs within or outside the main coseismic rupture patches (e.g. Freed, 2007; Kositsky and Avouac, 2010; Evans and Meade, 2012). Accordingly, we need to develop appropriate inversion methods to estimate time-dependent afterslip while also considering viscoelastic responses to coseismic and postseismic stress changes.

Fukahata et al. (2004) developed a framework for geodetic data inversion using viscoelastic Green's functions and taking advantage of Akaike's Bayesian Information Criterion (ABIC). The framework performs the inversion by expanding the slip with respect to basis functions and regularizing the solution using two hyperparameters.

Kositsky and Avouac (2010) demonstrated how to invert geodetic time series without requiring additional hyperparameters using the inverse of the elastic Green's function $\left(G^{-1}\right)$ multiplied by a singular value decomposition (SVD) of the ground observations $(D)$. In this approach, referred to as the Principal Component Analysis-based Inversion Method (PCAIM), information about time-dependent slip is obtained directly by decomposing the temporally dense geodetic observations into spatial $(U)$ and temporal $(V)$ functions:

$$
\begin{gathered}
D_{r}=U_{r} \Lambda_{r} V_{r}^{\prime} \\
S=G^{-1} U_{r}\left(\Lambda_{r} V_{r}^{\prime}\right)
\end{gathered}
$$

Here $S$ is a time-dependent slip matrix; $r$ is the number of singular values retained; $D_{r}$ ( $n \times T$ dimensions) is a filtered form of the full displacement matrix $D$ with $n$ observed components at $T$ epochs; $U_{r}(n \times r)$ represents the first $r$ columns of $U ; \Lambda_{r}(r \times r)$ is formed from the first $r$ rows and columns of $\Lambda$, which contains singular values of $D$; and $V_{r}(T \times r)$ represents the first $r$ columns of $V$. The optimal value of $r$ is selected by minimising the misfit between the displacement signal $D$ and the filtered signal $D_{r}$. In this chapter, we adapt the PCAIM method of Kositsky and Avouac (2010) to invert geodetic time series in the case of an elastic-viscoelastic medium. This approach enables us to accommodate either semi-analytical (Fukahata and Matsu'ura, 2006) or hybrid numerical viscoelastic Green's functions (Aagaard et al., 2009, 2013, 2015). 
Table 4.1: Table of nomenclature

\begin{tabular}{c||c}
\hline Symbol & Explanation \\
\hline $\mathrm{n}$ & Number of displacement components \\
$\mathrm{m}$ & Number of slip components \\
$\mathrm{T}$ & Number of epochs \\
$\vec{d}$ & Displacement vector minus coseismic viscoelastic response \\
$\overrightarrow{\hat{d}}$ & Total postseismic displacement vector \\
$\overrightarrow{d c}$ & Displacement vector of coseismic viscoelastic response \\
$D$ & Temporal displacement matrix \\
$\mathrm{g}$ & Matrix of Green's functions \\
$\mathrm{G}$ & Matrix of temporal Green's functions \\
$\widetilde{G}$ & unit-step slip vector \\
$\vec{s}$ & Slip rate vector \\
$\vec{v}$ & Temporal slip matrix \\
$S$ & Decomposed space function of $X$ \\
$U_{X}$ & Diagonal singular values of $X$ \\
$\Lambda_{X}$ & Decomposed time function of $X$ \\
$V_{X}$ & The transpose of a matrix $X$ \\
$V_{X}^{t}$ & Scaled decomposed time function of $X\left(\Lambda_{X} V_{X}\right)$ \\
$X^{\prime}$ & Matrix of decomposed viscoelastic time function \\
$\widetilde{V}_{v}$ & Matrix of decomposed elastic time function \\
$\widetilde{V}_{e}$ & \\
\hline
\end{tabular}

\subsection{Methods}

\subsubsection{Mathematical framework}

In order to calculate viscoelastic effects, we must first compute appropriate viscoelastic Green's functions. Fukahata and Matsu'ura (2006) used the semi-analytical propagator matrix method to compute viscoelastic Green's functions for a dislocation source embedded in a multi-layered elastic-viscoelastic half-space, taking into consideration the gravity field. It is also possible to compute the equivalent viscoelastic Green's functions numerically, which we do here using the PyLith code (Aagaard et al., 2009, 2013, 2015). Table 4.1 lists the parameters referred to in the analysis below.

Postseismic displacements can be split into elastic and viscoelastic components. At time $t$, the elastic-viscoelastic displacement $\overrightarrow{\hat{d}}$ in an arbitrary direction is presumed to be expressed by the superposition of responses to unit-step slip $(\vec{s})$ occurring at time $\tau<t$. Accordingly,

$$
\overrightarrow{\hat{d}}(t)=\vec{d}^{c}(t)+\int_{0}^{t} G(t-\tau) \vec{v}(\tau) d \tau
$$


where $G$ is the matrix of elastic-viscoelastic Green's functions and $\vec{v}$ is the afterslip rate. By discretising the integral, we approximate Equation 4.3 as

$$
\overrightarrow{\hat{d}}\left(t_{j}\right) \simeq \overrightarrow{d^{c}}\left(t_{j}\right)+\sum_{k=1}^{j-1} G\left(t_{j}-t_{k}\right) \vec{s}_{k}
$$

In both Equations 4.3 and 4.4, the superscript $c$ indicates the viscoelastic displacement in response to coseismic stress changes.

Our approach here is to compute the viscoelastic response to the coseismic event and then subtract this from the observed displacement to leave $\vec{d}$ (the second term in Equations 4.3 and 4.4). Equation 4.4 can then be rewritten as

$$
\begin{aligned}
\vec{d}_{n \times 1}^{1} & =G_{n \times m}^{1} \vec{s}_{m \times 1}^{1} \\
\vec{d}_{n \times 1}^{2} & =G_{n \times m}^{2} \vec{s}_{m \times 1}^{1}+G_{n \times m}^{1} \vec{s}_{m \times 1}^{2} \\
\vec{d}_{n \times 1}^{3} & =G_{n \times m}^{3} \vec{s}_{m \times 1}^{1}+G_{n \times m}^{2} \vec{s}_{m \times 1}^{2}+G_{n \times m}^{1} \vec{s}_{m \times 1}^{3} \\
\quad & \\
\vec{d}_{n \times 1}^{T} & =G_{n \times m}^{T} \vec{s}_{m \times 1}^{1}+G_{n \times m}^{T-1} \vec{s}_{m \times 1}^{2}+\cdots \cdots \cdots+G_{n \times m}^{1} \vec{s}_{m \times 1}^{T}
\end{aligned}
$$

where $n$ is the number of displacement components and equals the number of stations multiplied by the number of dimensions (horizontal and vertical), $m$ the number of slip components on the fault plane, and $T$ is the number of epochs. $G_{n \times m}^{1}$ represents the viscoelastic Green's functions after one epoch, $G_{n \times m}^{2}$ after two epochs and similarly $G_{n \times m}^{T}$ after $T$ epochs. Green's functions incorporate both elastic $\left(G_{e}\right)$ and viscoelastic $\left(G_{v}\right)$ responses to slip as

$$
G_{n \times m}=G_{v n \times m}+G_{e n \times m}
$$

In contrast with the impulsive elastic response, viscoelastic Green's functions are timedependent. Using SVD of the viscoelastic Green's functions, the time functions can be decoupled from the space functions. We consider the matrix $\widetilde{G}_{v}$ consisting of the viscoelastic Green's functions from epoch 1 to epoch $T$.

$$
\widetilde{G}_{v(n \times m) T}=\left[\begin{array}{lllll}
g_{v(n \times m) 1}^{1} & g_{v(n \times m) 1}^{2} & g_{v(n \times m) 1}^{3} & \cdots & g_{v(n \times m) 1}^{T}
\end{array}\right]
$$

Here $g_{v}$ is the vector form of the viscoelastic Green's functions $\left(G_{v}\right)$ at a particular time. The vector $g_{v}$ in time is a function of one or more values chosen for viscosity or relaxation time and the rheological models.

In this study, we use reduced SVD (Kawamura and Yamaoka, 2006, 2009), in which it is sufficient to represent $\widetilde{G}_{v}$ at $r=1$ levels. Assuming more complex geometries, other rheologies and heterogeneity of elastic and viscoelastic materials may impose more than one spatial and temporal mode. Whether or not a reduced number of one $(r=1)$ is sufficient to precisely decompose the Green's functions is a subject of ongoing research. 
With this assumption, we can express the viscoelastic Green's function as

$$
\begin{gathered}
\widetilde{G}_{v}=U_{\widetilde{G}_{v}(n \times m) \times r} \Lambda_{\widetilde{G}_{v} r \times r} V_{\widetilde{G}_{v} r \times T}^{\prime} \\
V_{\widetilde{G}_{v} r \times T}^{t^{\prime}}=\Lambda_{\widetilde{G}_{v} r \times r} V_{\widetilde{G}_{v} r \times T}^{\prime}
\end{gathered}
$$

Here $U_{\widetilde{G}_{v}}$ is the matrix of space functions (spatial mode), $\Lambda_{\widetilde{G}_{v}}$ is the diagonal matrix of singular values associated with $\widetilde{G}_{v}$, and $V_{\widetilde{G}_{v}}^{\prime}$ is the matrix of time functions (temporal modes). We use symbols $U, \Lambda$ and $V$ to represent space functions, scale values and time functions throughout this chapter.

We now revise Equation 4.5 using Equation 4.8 to obtain the associated elastic displacement $\overrightarrow{d_{e}}$

$$
\begin{aligned}
\vec{d}_{e n \times 1}^{1} & =G_{e}\left(\vec{s}_{m \times 1}^{1}\right) \\
\vec{d}_{e n \times 1}^{2} & =G_{e}\left(\vec{s}_{m \times 1}^{1}+\vec{s}_{m \times 1}^{2}\right) \\
\vec{d}_{e n \times 1}^{3} & =G_{e}\left(\vec{s}_{m \times 1}^{1}+\vec{s}_{m \times 1}^{2}+\vec{s}_{m \times 1}^{3}\right) \\
\quad & \\
\vec{d}_{e n \times 1}^{T} & =G_{e}\left(\vec{s}_{m \times 1}^{1}+\vec{s}_{m \times 1}^{2}+\cdots \cdots \cdots+\vec{s}_{m \times 1}^{T}\right)
\end{aligned}
$$

and viscoelastic displacement $\vec{d}_{v}$

$$
\begin{aligned}
\vec{d}_{v n \times 1}^{1} & =\sum_{i=1}^{r} U_{\widetilde{G}_{v}}^{i}\left(v_{i}^{1} \vec{s}_{m \times 1}^{1}\right) \\
\vec{d}_{v n \times 1}^{2} & =\sum_{i=1}^{r} U_{\widetilde{G}_{v}}^{i}\left(v_{i}^{2} \vec{s}_{m \times 1}^{1}+v_{i}^{1} \vec{s}_{m \times 1}^{2}\right) \\
\vec{d}_{v n \times 1}^{3} & =\sum_{i=1}^{r} U_{\widetilde{G}_{v}}^{i}\left(v_{i}^{3} \vec{s}_{m \times 1}^{1}+v_{i}^{2} \vec{s}_{m \times 1}^{2}+v_{i}^{1} \vec{s}_{m \times 1}^{3}\right) \\
\vdots & \\
\vec{d}_{v n \times 1}^{T} & =\sum_{i=1}^{r} U_{\widetilde{G}_{v}}^{i}\left(v^{T} \vec{s}_{m \times 1}^{1}+v^{T-1} \vec{s}_{m \times 1}^{2}+\cdots \cdots \cdots \cdots+v^{1} \vec{s}_{m \times 1}^{T}\right)
\end{aligned}
$$

where $v_{i}^{1}$ is calculated by multiplication of the $i^{\text {th }}$ singular value and the decomposed time function of viscoelastic Green's functions at the first epoch, $v_{i}^{2}$ at the second epoch and $v_{i}^{T}$ at the $T_{t h}$ epoch.

The time-dependent slip matrix $(S)$, elastic $\left(D_{e}\right)$ and viscoelastic $\left(D_{v}\right)$ matrices of displacement can now be expressed as follows

$$
\begin{aligned}
S & =\left[\begin{array}{llll}
\vec{s}^{1} & \vec{s}^{2} & \ldots & \vec{s}^{T}
\end{array}\right] \\
D_{e} & =\left[\begin{array}{llll}
\vec{d}_{e}^{1} & \vec{d}_{e}^{2} & \cdots & \vec{d}_{e}^{T}
\end{array}\right] \\
D_{v} & =\left[\begin{array}{llll}
\vec{d}_{v}^{1} & \vec{d}_{v}^{2} & \cdots & \vec{d}_{v}^{T}
\end{array}\right]
\end{aligned}
$$


Accordingly, from equations 4.9 and 4.10, we can express the elastic-viscoelastic displacement in matrix forms as

$$
D_{e}=G_{e} S \widetilde{V}_{e}
$$

and

$$
D_{v}=\sum_{i=1}^{r} U_{\widetilde{G}_{v}}^{i} S \widetilde{V}_{v}^{i}
$$

or after decomposing the slip matrix $\mathrm{S}$, we have

$$
D_{e}=G_{e} U_{S} V_{S}^{t} \widetilde{V}_{e}
$$

and

$$
D_{v}=\sum_{i=1}^{r} U_{\widetilde{G}_{v}}^{i} U_{S} V_{S}^{t} \widetilde{V}_{v}^{i}
$$

Space and time functions of the slip matrix are computed from the elastic displacement matrix ( $D_{e}$; Kositsky and Avouac, 2010), namely

$$
\begin{aligned}
U_{S} & =\left(G_{e}\right)^{-1} U_{e} \\
V_{S}^{t} & =V_{e}^{t}\left(\widetilde{V}_{e}\right)^{-1}
\end{aligned}
$$

Consequently, the relationships between elastic and viscoelastic space and time functions are as follows

$$
\begin{aligned}
U_{v}^{i} & =U_{\widetilde{G}_{v}}^{i}\left(G_{e}\right)^{-1} U_{e} \\
V_{v}^{t ; i} & =V_{e}^{t}\left(\widetilde{V}_{e}\right)^{-1}\left(\widetilde{V}_{v}^{i}\right)
\end{aligned}
$$

where

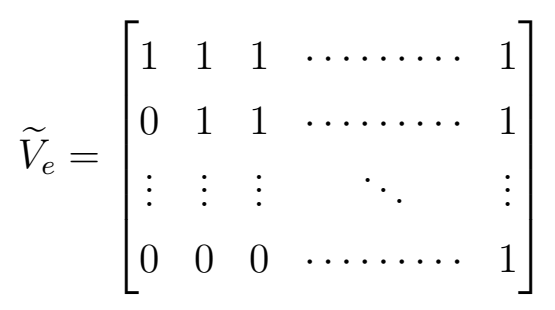

and

$$
\widetilde{V}_{v}=\left[\begin{array}{ccccc}
v_{i}^{1} & v_{i}^{2} & v_{i}^{3} & \ldots \ldots \ldots & v_{i}^{T} \\
0 & v_{i}^{1} & v_{i}^{2} & \ldots \ldots \ldots & v_{i}^{T-1} \\
\vdots & \vdots & \vdots & \ddots & \vdots \\
0 & 0 & 0 & \ldots \ldots \ldots & v_{i}^{1}
\end{array}\right]
$$

The overall displacement is the sum of both displacement components, elastic and 
viscoelastic:

$$
D=D_{e}+D_{v}
$$

After rearrangement

$$
D_{v}=\sum_{i=1}^{r} U_{\widetilde{G}_{v}}^{i}\left(G_{e}\right)^{-1} D_{e}\left(\widetilde{V}_{e}\right)^{-1}\left(\widetilde{V}_{v}^{i}\right)
$$

such that

$$
D=D_{e}+\sum_{i=1}^{r} U_{\widetilde{G}_{v}}^{i}\left(G_{e}\right)^{-1} D_{e}\left(\widetilde{V}_{e}\right)^{-1}\left(\widetilde{V}_{v}^{i}\right)
$$

where ()$^{-1}$ denotes the regularised inverse of a matrix.

With further rearrangement, Equation 4.26 can be formulated as a Sylvester equation (Sylvester, 1884) of the form $C=A X+X B$, for which a unique solution exists if and only if $A$ and $B$ have disjoint eigenvalues (Bartels and Stewart, 1972). We simplify Equation 4.26 by assuming $r=1$ for in order to decouple the viscoelastic Green's functions into spatial and temporal modes. Equation 4.27 is expressable in terms of spatial and temporal modes of elastic displacement.

$$
D=U_{e} V_{e}^{t}+U_{\widetilde{G}_{v}}\left(G_{e}\right)^{-1} U_{e} V_{e}^{t}\left(\widetilde{V}_{e}\right)^{-1}\left(\widetilde{V}_{v}\right)
$$

Keeping variable $U_{e}$ constant

$$
A=U_{e} ; B=U_{\widetilde{G}_{v}}\left(G_{e}\right)^{-1} U_{e} \equiv U_{\widetilde{G}_{v}} U_{S} ; C=\left(\widetilde{V}_{e}\right)^{-1}\left(\widetilde{V}_{v}\right)
$$

thus

$$
D=A V_{e}^{t}+B V_{e}^{t} C
$$

\subsubsection{Iterative Decoupling of Afterslip and Viscoelastic relax- ation (IDAV)}

We estimate the elastic spatial function $\left(U_{e}\right)$ in Equation 4.27 from decomposition of the observed displacement signal, and treat this parameter as unchanging in our inversion. This assumption reduces the task of solving the Sylvester equation to one of iteratively updating the temporal elastic time function $V_{e}$. Methods of solving the Sylvester equation in which $U_{e}$ and $V_{e}$ are estimated simultaneously will be left for further research.

In order to regularise Equation 4.29, we take advantage of Laplacian smoothing in space and decomposition into separate space and time functions. Solving Equation 4.29 yields 
a solution for the time functions of elastic displacement.

The overall method of solving Equation 4.29 is illustrated in Figure 4.1. The expected viscoelastic response to coseismic stress changes can be calculated using known (or assumed) viscoelastic properties, specifically viscosity and the thickness of the elastic layer in a half-space model, in conjunction with the coseismic slip distribution. We start by subtracting this response and centering the displacement time series about zero. Thereafter, the remainder encompasses afterslip and the associated viscoelastic response. We apply SVD to the remaining signal in order to extract the elastic part of the signal from the total displacement. Regular (unconstrained) decomposition can over- or underestimate the elastic part of the signal and disrupt convergence of the proposed method.

Equation 4.17 implies that the viscoelastic displacement in time is a function of timedependent afterslip $\left(V_{S}^{t}\right)$ and time-dependent viscoelastic Green's functions $\left(\widetilde{V_{v}^{i}}\right)$. Timedependent Green's functions are calculated based on the geometry of the region and the fault surface, viscosity and the rheology of the viscoelastic medium. Green's functions vary by changing the geometry of the problem and/or assuming multiple layers with different elastic and viscoelastic properties. Time-dependent afterslip is yet to be determined, meaning that the time functions of viscoelastic displacement are unknown. However, the domain of the viscoelastic time functions spans all possibilities of viscoelastic response to slip with relaxation times between zero $(\tau=0)$ and infinity $(\tau=\infty)$.

The faster the afterslip decays, the closer the viscoelastic displacement time functions become to the end-member $\tau=0$. The temporal mode of the end-member $\tau=0$ corresponds with the temporal mode of the viscoelastic Green's functions $\left(\widetilde{V_{v}^{i}}\right)$. Accordingly, such a temporal mode of viscoelastic response can be imposed as one component of the time functions in the decomposition method, if unconstrained decomposition does not apply. We approximate the space functions of elastic displacement $\left(U_{e}\right)$ with the result of constrained decomposition by imposing $\widetilde{V_{v}^{i}}$ ( $\equiv$ end-member $\left.\tau=0\right)$ or the first component of the unconstrained decomposition. The next step in the calculation is to remove the remaining viscoelastic response from the estimated elastic component and to add it back to the viscoelastic spatial and temporal functions.

To summarize, we solve Equation 4.29 with an iterative block linear method (Miller, 1988 ) for time functions of elastic displacement $\left(V_{e}\right)$. We invert the elastic space functions $\left(U_{e}\right)$ using Laplacian smoothing. We keep variable $U_{e}$ constant and therefore iteratively we update the variable $V_{e}$ to find a solution for Equation 4.29, which also corresponds to Equation 4.26. The initial elastic time functions are set to $V_{e}$ obtained 


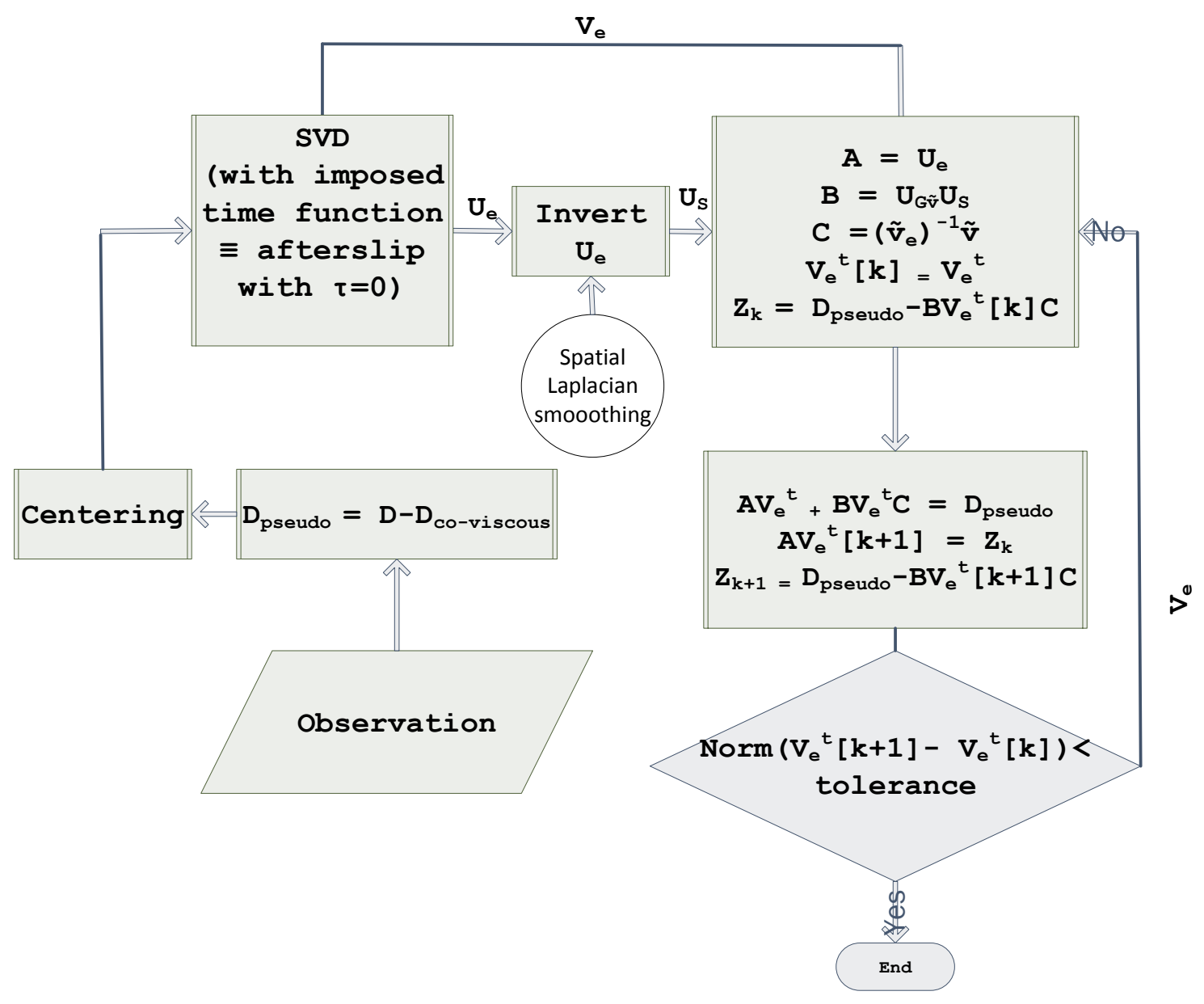

Figure 4.1: Methodology used to obtain unique solutions for the elastic-viscoelastic Sylvester equations 4.26 and 4.29. In the flowchart we show how to decouple elastic from viscoelastic time functions.

from decomposition. Thereafter we solve for an equation in a form of $A V_{e}^{t}(k+1)=Z(k)$ where $Z(k)=D-B V_{e}^{t}(k) C$ at iteration $k$. We then update $Z(k+1)$ using the updated $V_{e}^{t}(k+1)$. The algorithm terminates once norm $\left[V_{e}^{t}(k+1)-V_{e}^{t}(k)\right]<$ tolerance value and the required correction to the elastic time functions are performed (Figure 4.1). We call this framework of solving the elastic-viscoelastic equation "Iterative Decoupling of Afterslip and Viscoelastic relaxation (IDAV)" method. 


\subsection{Simulating postseismic displacement using an- alytical Green's functions}

To demonstrate the utility of this method, we first simulate deformation in the case of an elastic layer with a thickness of $35 \mathrm{~km}$ overlying a viscoelastic half-space with a viscosity value of $10^{18} \mathrm{~Pa} \cdot \mathrm{s}$ and a constant shear modulus of $30 \mathrm{GPa}$. In this simulation, exponentially decaying afterslip occurs within two patches with a maximum accumulated slip of $2.5 \mathrm{~m}$ down-dip of the coseismic fault plane, striking $023^{\circ}$ and dipping between $013^{\circ}$ and $050^{\circ}$ towards the south-east. Displacements due to afterslip (red; $\vec{d}_{e}$ ), the viscoelastic response to afterslip (green; $\vec{d}_{v}$ ) and the viscoelastic response to imposed coseismic rupture (blue; $\overrightarrow{d^{c}}$ ) at each of 40 sites are computed using elastic (Okada, 1985) and viscoelastic Green's functions (Fukahata and Matsu'ura, 2006; Figure 4.2).

We infer from the simulated data for this geometry and the location of sites that the viscoelastic responses to coseismic rupture and to afterslip can occur in different directions. The arrows illustrate the accumulated displacement within 400 days of the simulated rupture. In the time series generated at three representative sites, the total displacement is depicted in black. Note that the associated names do not correspond to real cGPS sites.

We next invert the calculated total displacement $(\vec{d})$ to reproduce the simulated afterslip model. In Figure 4.3a, we decompose the total displacement into two components using the constrained (solid lines) and unconstrained (dashed lines) decomposition methods (see Section 4.1; Equation 4.1). Unconstrained decomposition yields correlated components and consequently underestimates the first component (blue). In order to avoid this, we decompose the signal with a viscoelastic time function corresponding to slip with relaxation time of zero $(\tau=0$; Figure $4.3 \mathrm{~b}$, red). The viscoelastic time function equivalent to constant slip with infinite relaxation time is illustrated in blue in Figure 4.3b as the upper bound of the viscoelastic temporal mode $(\tau=\infty)$.

The iterative method presented in Figure 4.1 is followed until convergence, which in this case occurs after three iterations when norm $\left[V_{e}^{t}(k+1)-V_{e}^{t}(k)\right]<1 \mathrm{~mm}$. In Figure $4.3 \mathrm{c}$ and $\mathrm{d}$, the initial and final elastic and viscoelastic time functions are presented in blue and red, respectively. The estimated slip after convergence is illustrated in Figure 4.4a in comparison to the originally imposed slip in Figure 4.4b, within 400 days of the simulated rupture. We also illustrate horizontal and vertical fits (blue) to the simulated elastic displacements (red) between one and 400 days of the simulated rupture, in 

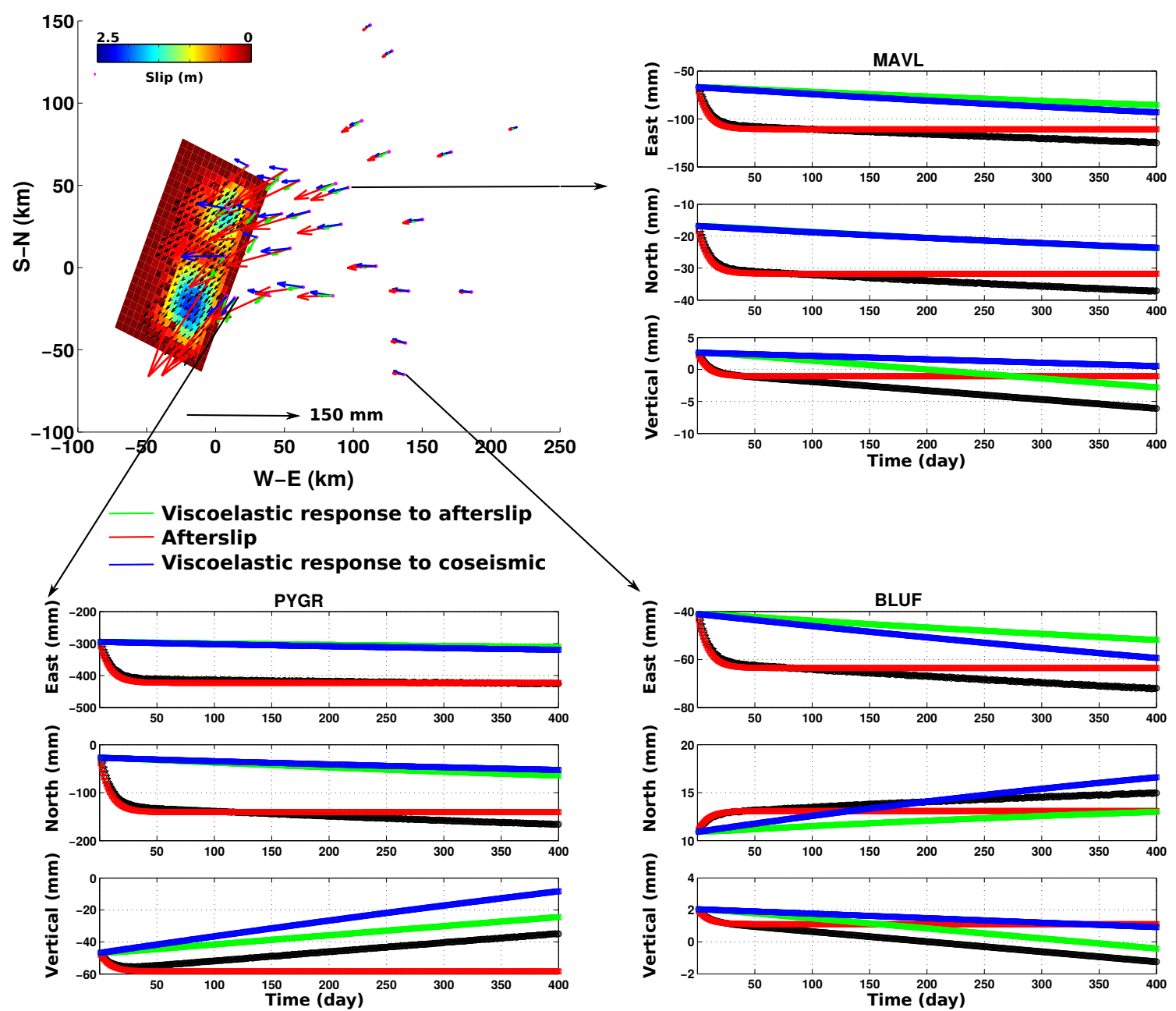

Figure 4.2: The accumulated displacement within 400 days of a simulated rupture due to afterslip (red), the viscoelastic response to afterslip (green) and the viscoelastic response to coseismic slip (blue). Corners: Time series of different components of simulated displacement illustrated for three sites. Black lines show the total displacement time series. 

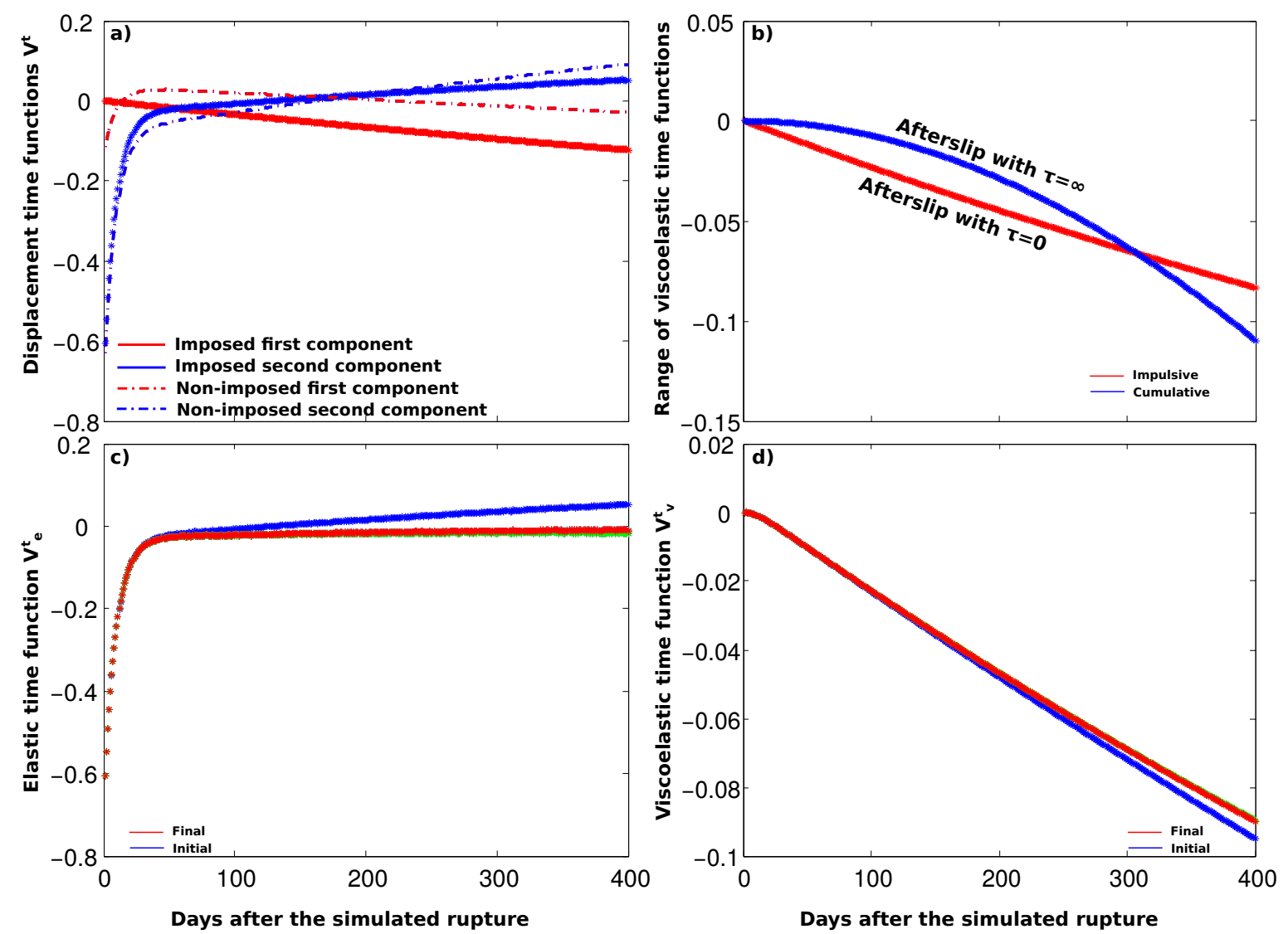

Figure 4.3: a) Time functions resulting from constrained (solid lines) and unconstrained (dashed lines) decomposition methods. b) Domain of viscoelastic time functions from a response to afterslip with relaxation time of zero (red) and infinity (blue). c) Convergence of elastic time functions from blue to red. d) Convergence of viscoelastic time functions from blue to red.

Figures $4.5 \mathrm{a}$ and $\mathrm{b}$.

\subsection{Postseismic displacement following the $\mathrm{M}_{\mathrm{W}} 7.8$ Dusky Sound earthquake, Fiordland, New Zealand}

Postseismic displacement following the Dusky Sound earthquake and associated Coulomb stress changes and strain accumulation in the time period between the 15 July 2009 Dusky Sound and 4 September 2010 Darfield earthquakes have been calculated in Chapter 3. We illustrate focal mechanisms of the Dusky Sound and Darfield earthquakes, the region of study, the locations of cGPS sites and cGPS displacement times series at the two sites nearest to the epicentral area in Figure 4.6.

In Chapter 3, we explained the postseismic displacement observed with continuous GPS and early campaign GPS data acquired during August 2009 and Differential Interfero- 


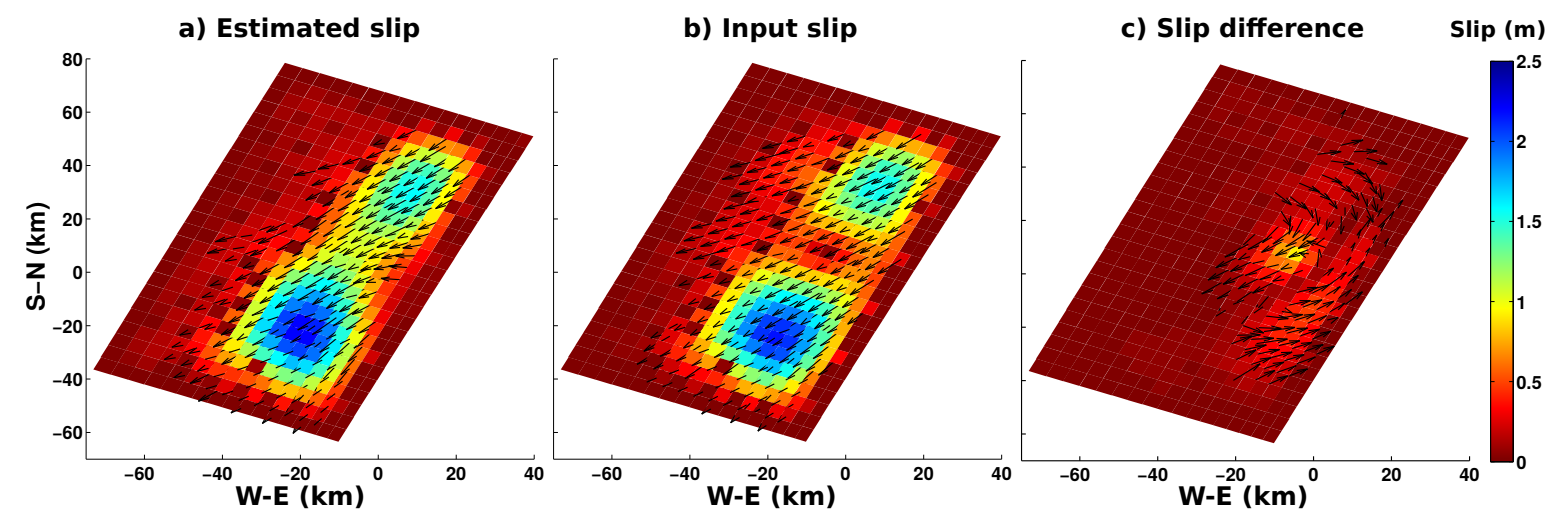

Figure 4.4: a) Estimated slip accumulated 400 days after coseismic rupture b) Simulated slip used as input in order to produce the displacement time series c) Difference between estimated and simulated slip.
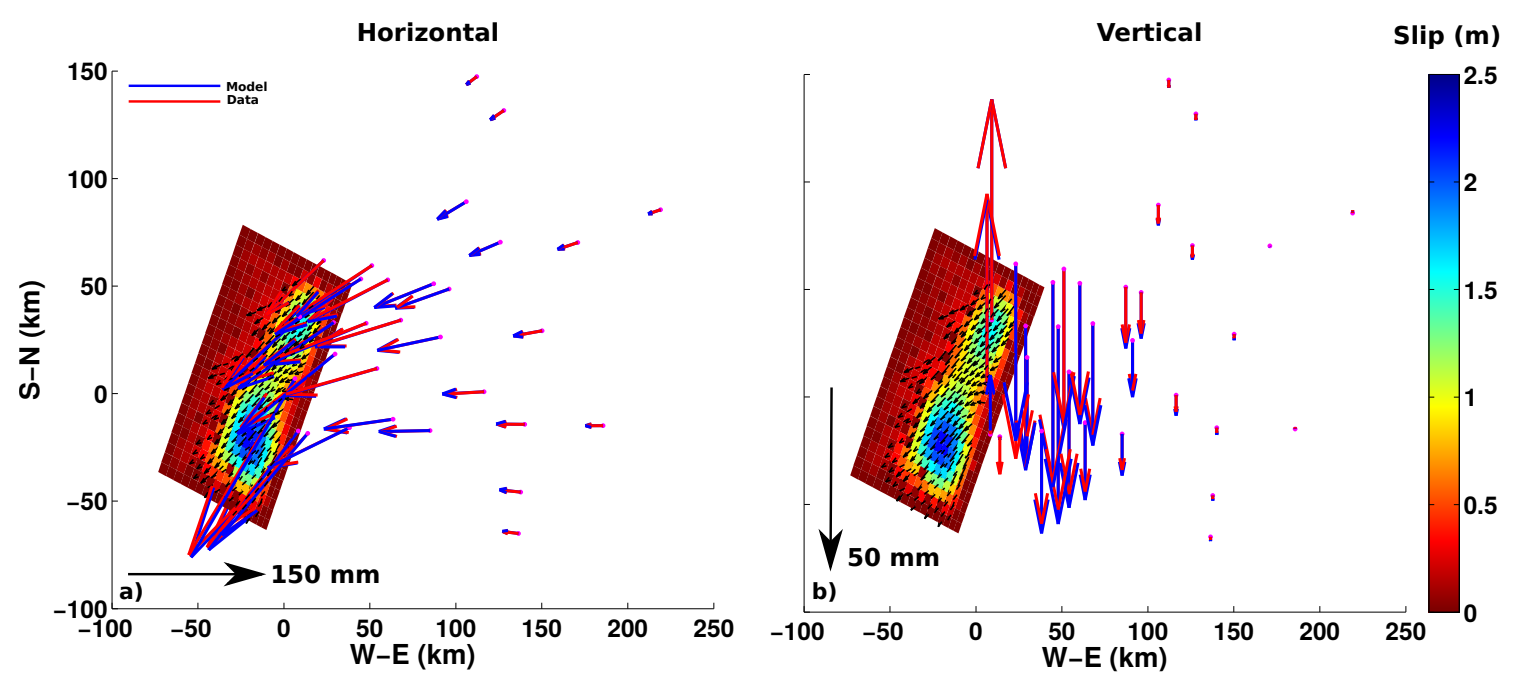

Figure 4.5: a) Horizontal and b) Vertical fits (blue) to the simulated elastic displacement (red) between day one and day 400 following the simulated rupture. 

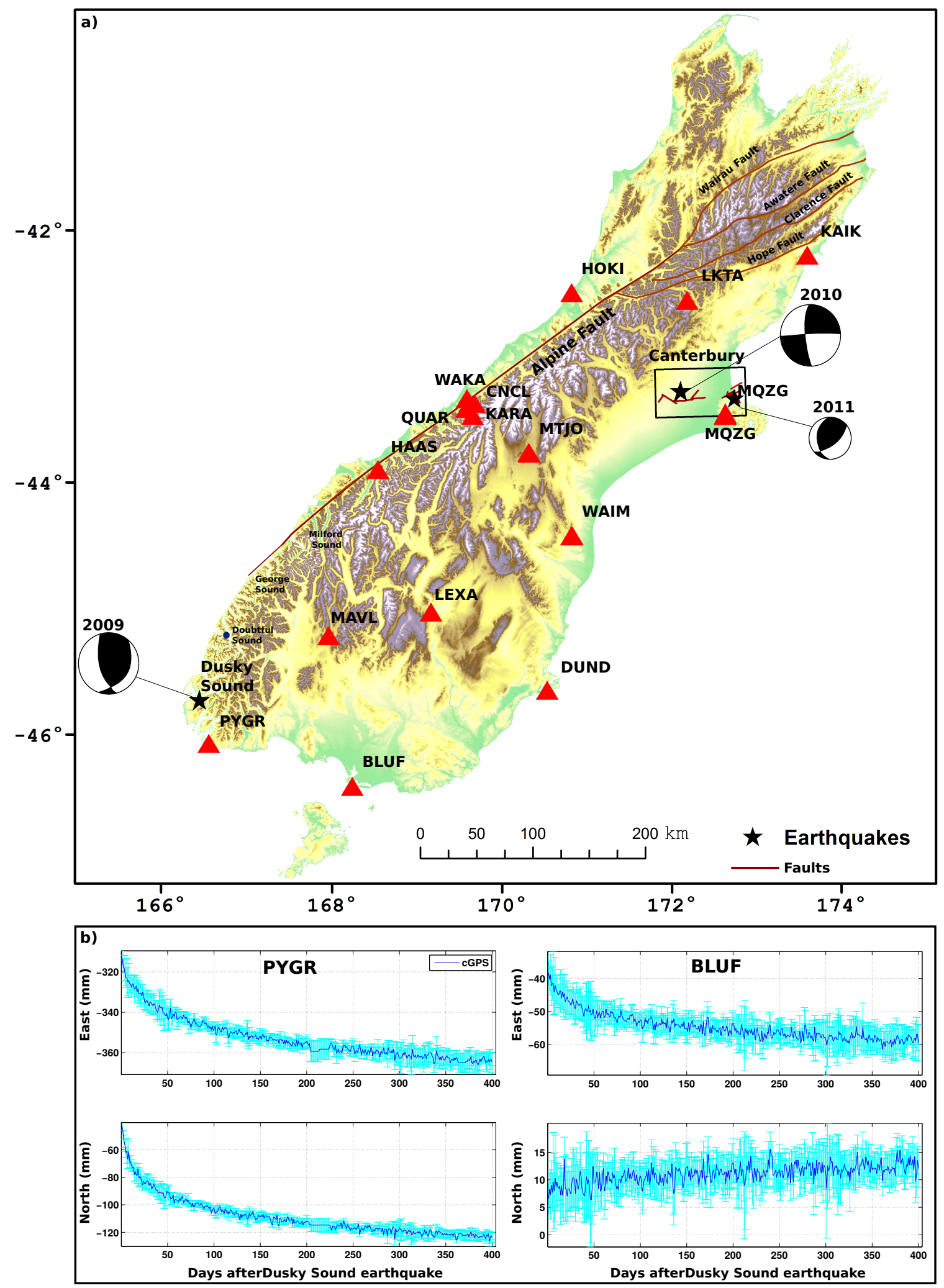

Figure 4.6: a) Focal mechanisms of recent earthquakes, namely the 15 July 2009 Dusky Sound, 4 September 2010 Darfield and 22 February 2011 Christchurch earthquakes, which were widely felt in the South Island, New Zealand. Locations of cGPS sites are shown in red triangles. b) Time series of cGPS displacement following the Dusky Sound earthquake at two nearest cGPS sites to the epicentral area. 


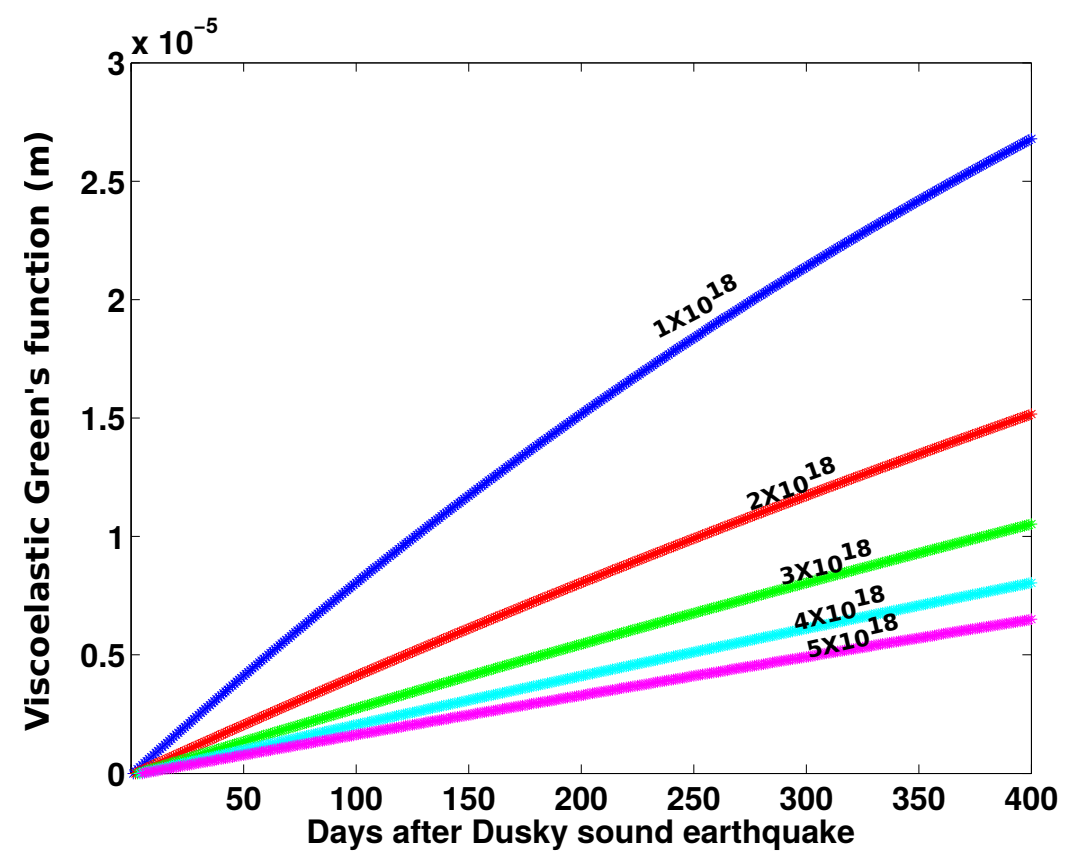

Figure 4.7: Viscoelastic Green's functions for a fault patch at an arbitrary point in time, with different values of viscosity. The cumulative displacement over one day for a Maxwell material with a viscosity of $10^{18} \mathrm{~Pa} \cdot \mathrm{s}$ is twice that of a viscosity of $2 \times 10^{18} \mathrm{~Pa} \cdot \mathrm{s}$

metric Synthetic Aperture Radar (DInSAR) observations in terms of coupled afterslip and viscoelastic relaxation. In this model, two patches on the fault surface (Beavan et al., 2010a) at depths of 0-80 km accommodated afterslip with a maximum slip of $\sim 1 \mathrm{~m}$ decaying logarithmically in the period between the Dusky Sound and Darfield earthquakes. The fault surface is embedded in an elastic layer with a thickness of 45 $\mathrm{km}$ overlying a Maxwell viscoelastic half-space with viscosities of $5 \times 10^{18}-10^{20} \mathrm{~Pa} \cdot \mathrm{s}$.

Here, lower values of viscosity than those considered in Chapter 3 , between $2 \times 10^{18}$ and $4 \times 10^{18} \mathrm{~Pa} \cdot \mathrm{s}$ for the Maxwell half-space, are examined using the IDAV method introduced in Section 4.2. Green's functions are calculated for the fault patches assuming a viscosity of $10^{18} \mathrm{~Pa} \cdot \mathrm{s}$ and using the semi-analytical propagator matrix methods of Fukahata and Matsu'ura (2006). The cumulative displacement over one day for a Maxwell material with a viscosity of $10^{18} \mathrm{~Pa} \cdot \mathrm{s}$ is twice that for a material with a viscosity of $2 \times 10^{18} \mathrm{~Pa} \cdot \mathrm{s}$ (Figure 4.7). Therefore, in order to reduce the computational time for higher-viscosity models, the Green's functions are computed by scaling the functions associated with a viscosity of $10^{18} \mathrm{~Pa} \cdot \mathrm{s}$.

In this case, unconstrained decomposition yields two uncorrelated components of displacement. Equation 4.29 converges after a few iterations with a tolerance of $1 \mathrm{~mm}$, for three values of viscosity. Elastic and viscoelastic time functions in the first and last iterations are presented in Figure 4.8a-c, from initial blue and cyan to final red and magenta, respectively. The total estimated afterslip models 400 days after the Dusky 
Table 4.2: Root Mean Square Errors, moment magnitudes and maximum slip values of coupled models for a Maxwell rheology with viscosity values of $2 \times 10^{18}, 3 \times 10^{18}$ and $4 \times 10^{18}$ $\mathrm{Pa} \cdot \mathrm{s}$.

\begin{tabular}{|c|ccc|}
\hline & \multicolumn{3}{|c|}{ Coupled models } \\
\hline Viscosity $(\mathbf{P a} \cdot \mathbf{s})$ & $2 \times 10^{18}$ & $3 \times 10^{18}$ & $4 \times 10^{18}$ \\
\hline RMSE $(\mathbf{m m})$ & 1.9 & 1.6 & 1.5 \\
Moment $\left(\times \mathbf{1 0}^{\mathbf{2 0}} \mathbf{N} \cdot \mathbf{m}\right)$ & 2.8 & 3.4 & 3.8 \\
Magnitude $\left(\mathbf{M}_{\mathbf{w}}\right)$ & 7.6 & 7.7 & 7.72 \\
Max slip $(\mathbf{m})$ & 0.86 & 1 & 1.1 \\
\hline
\end{tabular}

Sound earthquake are illustrated in Figure 4.8d-f for different viscosity values. Table 4.2 presents Root Mean Square Error (RMSE) values, the cumulative moment magnitude and the maximum values of slip for each coupled model with different viscosities. The higher the viscosity value, the larger the estimated afterslip. We can describe the difference between afterslip models for different values of viscosity with the effects of viscoelastic relaxation to partially explain the observed displacement signal.

The supplementary file contains the displacement time series estimated from the coupled model with a viscosity of $2 \times 10^{18}$ in red and observed time series in blue at 16 continuous GPS sites.

\subsection{Simulating postseismic displacement using nu- merical Green's functions}

We can also use the IDAV method of describing coupled viscoelastic relaxation and afterslip to simulate more complex faulting scenarios. We numerically simulate postseismic displacement following a $\mathrm{M}_{\mathrm{W}} 8.4$ subduction earthquake using the Finite Element Method (FEM) implemented in PyLith (Aagaard et al., 2009, 2013, 2015). The geometry for numerical simulation of the Green's functions consists of an elastic layer with a thickness of $50 \mathrm{~km}$ overlying a viscoelastic half-space (Figure 4.9). We also take into account an elastic slab with a thickness of $50 \mathrm{~km}$. We assume uniform elastic properties with a density of $2.5 \mathrm{~kg} / \mathrm{m}^{3}$, and $\mathrm{P}$ - and S-wave velocities of 5000 and 3000 $\mathrm{m} / \mathrm{s}$. We assign viscosities of $10^{18}$ and $5 \times 10^{18} \mathrm{~Pa} \cdot \mathrm{s}$ to the continental mantle and oceanic mantle, respectively (Figure 4.9). The north-south-striking fault, dipping $24^{\circ}$ westward, extends to the depth of $80 \mathrm{~km}$.

We mesh the geometry with a uniform resolution of $25 \mathrm{~km}$ using the software package Trelis (http://www.csimsoft.com/help/trelishelp.htm). We calculate Green's 

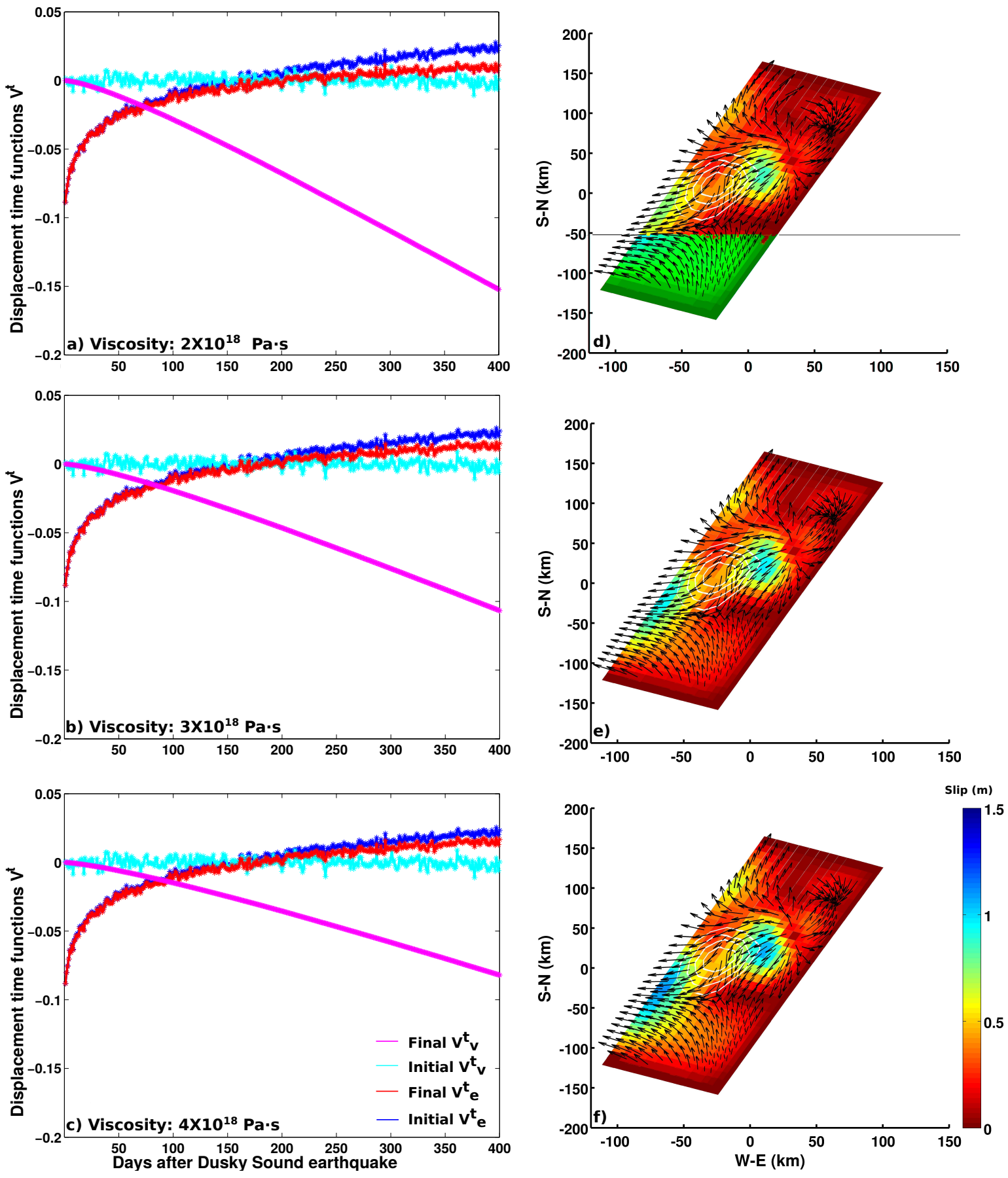

Figure 4.8: Convergence of elastic and viscoelastic time functions in first and last iterations from initial blue and cyan to final red and magenta, respectively a) Viscosity value of $2 \times 10^{18}$ $\mathrm{Pa} \cdot \mathrm{s}$ b) Viscosity value of $3 \times 10^{18} \mathrm{~Pa} \cdot \mathrm{s}$ c) Viscosity value of $4 \times 10^{18} \mathrm{~Pa} \cdot \mathrm{s}$. Accumulated slip 400 days after the Dusky Sound earthquake d) Viscosity value of $2 \times 10^{18} \mathrm{~Pa} \cdot \mathrm{s}$ e) Viscosity value of $3 \times 10^{18} \mathrm{~Pa} \cdot \mathrm{s}$ and $\mathrm{f}$ ) Viscosity value of $4 \times 10^{18} \mathrm{~Pa} \cdot \mathrm{s}$. The temporal accumulated slip in magnitude differs when we increase the viscosity from $2 \times 10^{18}$ to $4 \times 10^{18} \mathrm{~Pa} \cdot \mathrm{s}$. 


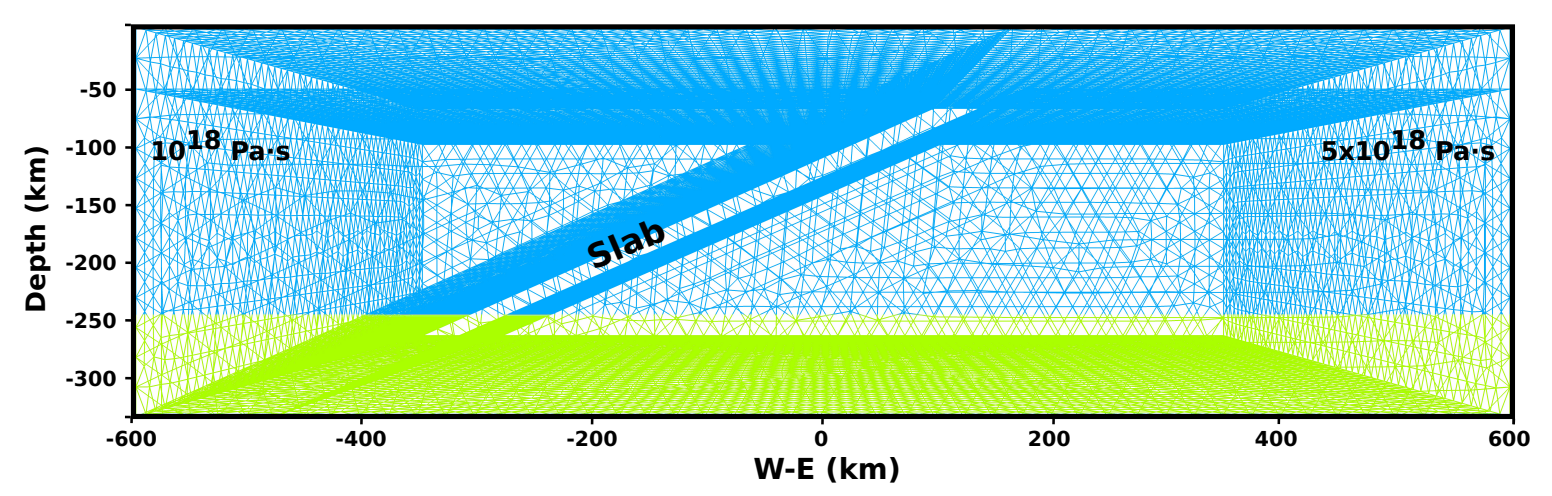

Figure 4.9: Viscoelastic half-space considering the slab geometry. Viscosities of $10^{18}$ and $5 \times 10^{18} \mathrm{~Pa} \cdot \mathrm{s}$ are assigned to the continental and oceanic mantle, respectively. The northsouth striking fault, dipping $24^{\circ}$ westward, extends to the depth of $80 \mathrm{~km}$ and the elastic slab has a thickness of $50 \mathrm{~km}$.

functions at each vertex, on a simulated fault, associated with 55 ground control points, as responses to unit strike- and dip-slip. Figure 4.10 illustrates unit-step slip at a vertex on the fault surface, which yields the Green's functions shown in the graph associated with a ground point (A1).

We compute the elastic (red) and viscoelastic (blue and green) components of displacement due to simulated time-dependent afterslip (Figure 4.11). Viscoelastic responses to the simulated rupture and afterslip are shown in blue and green, respectively and the total displacement in black. In Figure 4.11, we compare the elastic and viscoelastic components of displacement. We also illustrate time series of elastic component (red), viscoelastic responses to the simulated rupture (blue) and afterslip (green) in Figure 4.11.

We decompose the time-dependent displacement signals into spatial and time functions imposing the temporal mode of the Green's functions. The elastic and viscoelastic time functions converge to the final solutions (red and magenta, respectively) after five iterations with a threshold value of $1 \mathrm{~mm}$ (Figure 4.12). Initial elastic and viscoelastic time functions obtained from the constrained decomposition are shown in blue and cyan, respectively.

We show the simulated afterslip model in Figure 4.13a and the estimated afterslip model after inverting the displacement time series in Figures $4.13 \mathrm{~b}$. The discrepancy between simulated and estimated afterslip models is approximately $7 \%$ of the slip magnitude. 


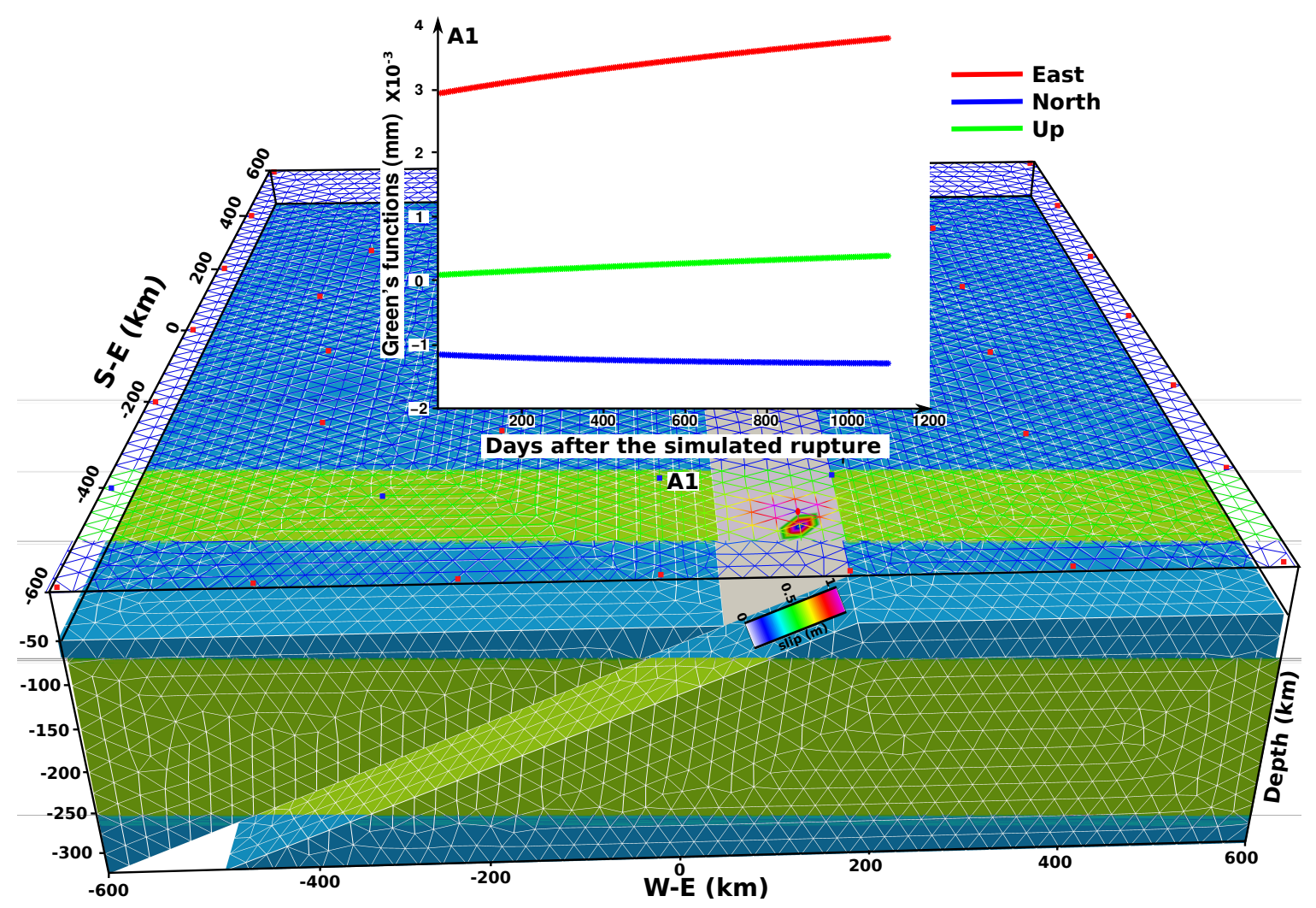

Figure 4.10: Numerically calculated Green's functions as responses to unit-step slip on a simulated fault. The north-south striking fault, dipping $24^{\circ}$ westward, extends to the depth of $80 \mathrm{~km}$ and the elastic slab has a thickness of $50 \mathrm{~km}$. 

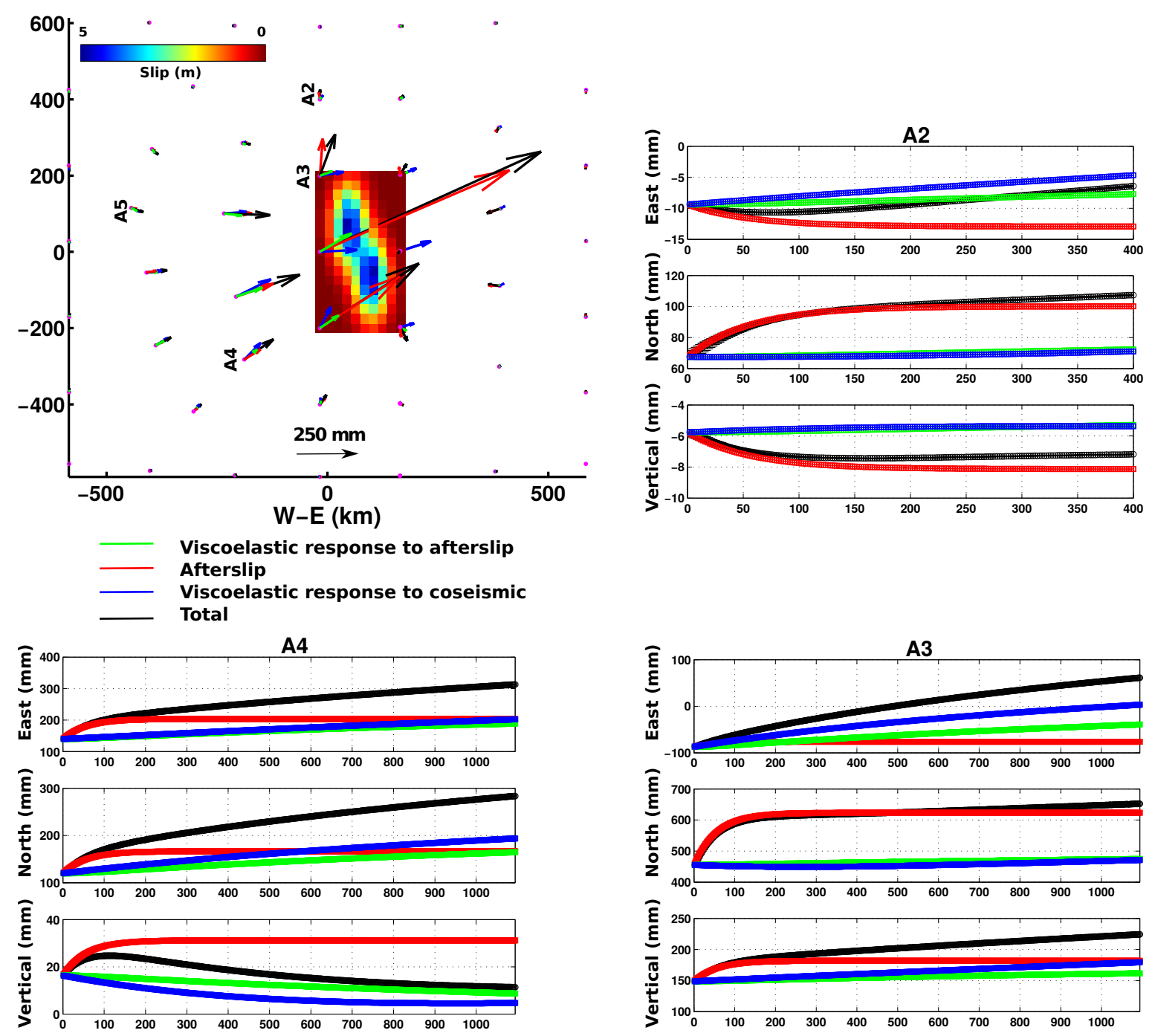

Figure 4.11: The accumulated displacement model of the slab geometry within 1095 days of a simulated subduction earthquake due to afterslip (red), viscoelastic response to afterslip (green) and viscoelastic response to coseismic slip (blue). Corners: Time series of different components of simulated displacement illustrated for three sites. 


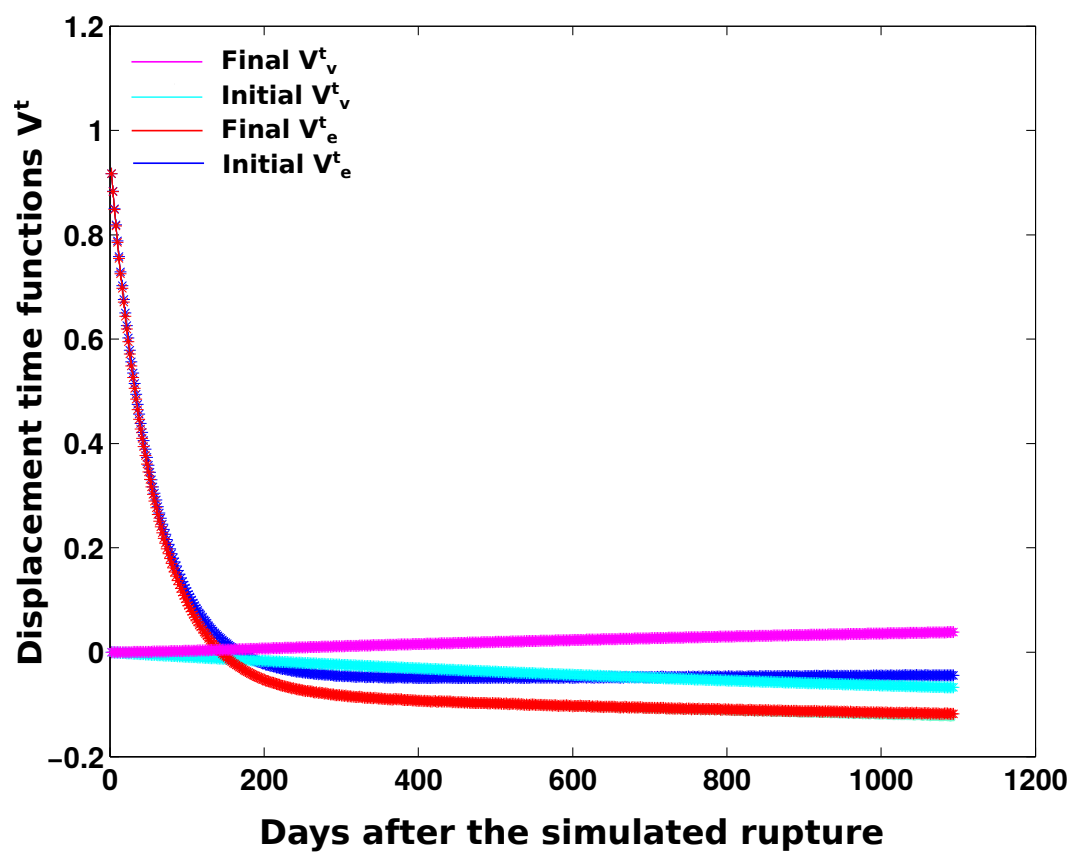

Figure 4.12: Elastic and viscoelastic time functions in different iterations from initial blue and cyan to final red and magenta, respectively.
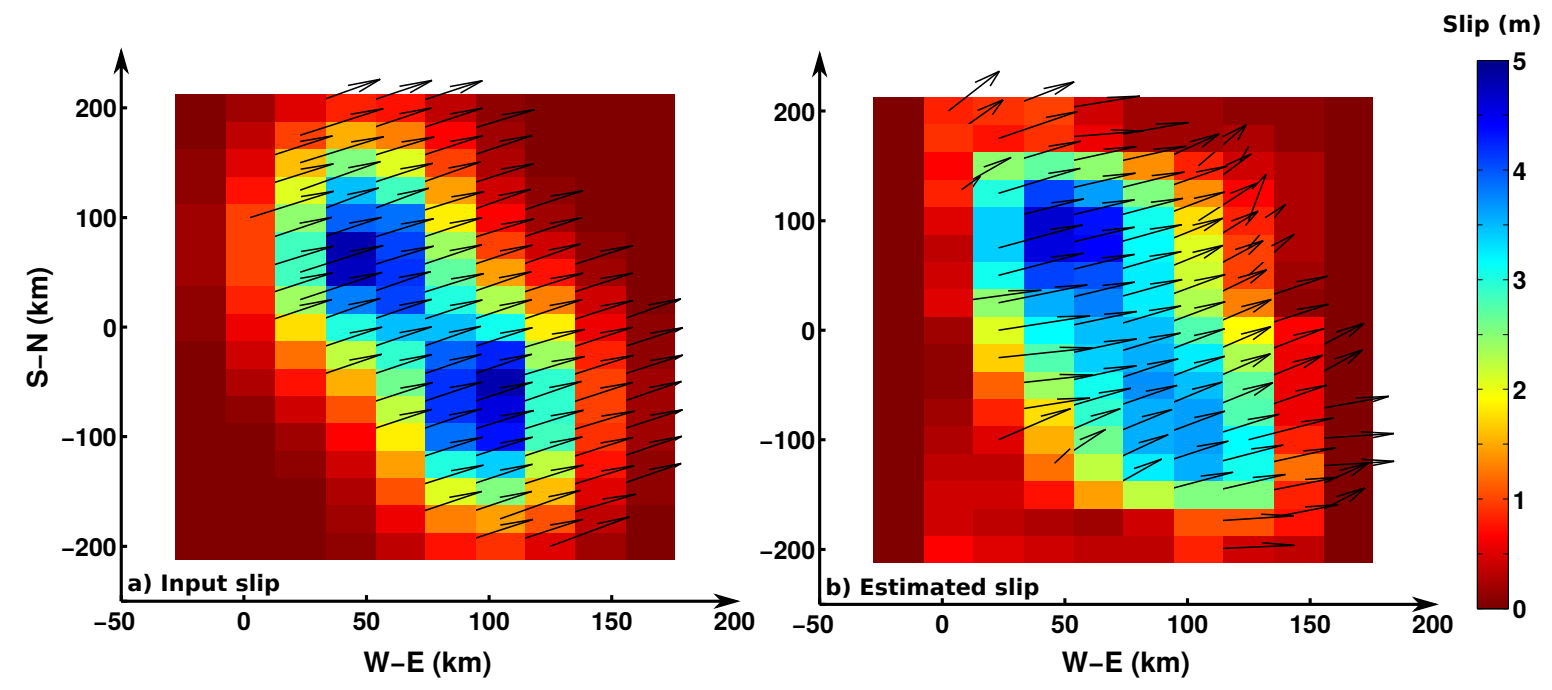

Figure 4.13: a) Simulated slip used as input in order to produce the displacement time series. b) Estimated slip from inverting the displacement time series induced by the slab geometry. 


\subsection{Discussion}

We have shown how to formulate the total postseismic displacement corresponding to elastic and viscoelastic components in terms of a Sylvester Equation 4.29 and developed a method of solving this equation in order to estimate each component (IDAV method).

The analytical and numerical simulations of postseismic displacement demonstrate that after large earthquakes, such as the 2009 Dusky Sound event, the viscoelastic response to afterslip can be significant. Within 400 days of the Dusky Sound earthquake, consideration of viscoelastic responses means that the moment magnitude of afterslip changes from $\mathrm{M}_{\mathrm{w}} 7.6$ to 7.7 , as the assumed viscosity varies from $2 \times 10^{18}$ to $4 \times 10^{18} \mathrm{~Pa} \cdot \mathrm{s}$. The difference between afterslip models for different viscosity values in this time interval are insignificant. In contrast, the effects of viscoelasticity do affect the longer-term displacement signal. Three years of numerically simulated postseismic displacement following a $\mathrm{M}_{\mathrm{W}} 8.4$ subduction earthquake reveals significant viscoelastic displacement compared to the displacement signal due to afterslip (see Figure 4.11).

In order to formulate the postseismic displacement in terms of separate elastic and viscoelastic responses, we have decomposed the Green's functions corresponding to the three examples considered into one set of spatial and time functions. Although sufficiently accurate for demonstrating the method's applicability, higher levels of Green's function decomposition should be assessed as part of further work.

For numerical simulations, the accuracy of the Green's functions is likely affected by two factors: 1) interpolating the Green's functions from the finite elements of the mesh onto the finite rectangular patches of the fault; 2) the residuals of displacement at the boundaries of the model domain. In an inversion framework for a real case study, a much larger model domain would be required to ensure accuracy. Assigning varying elastic properties to different parts of the model domain, and particularly to the slab, may result in different displacement models associated with the assumed geometry.

In this inversion method, we have assumed a priori knowledge of viscosity, rheology and the geometry of the viscoelastic half-space. Therefore, the validity of the solutions is contingent on the accuracy of these parameters, and in an ideal situation complementary data would be used to estimate the viscoelastic parameters. 


\subsection{Conclusions}

Postseismic displacement consisting of elastic and viscoelastic components can be expressed as a Sylvester equation, providing a novel means of estimating the time dependent slip distribution in an elastic-viscoelastic medium without introducing additional hyperparameters. The temporal modes of the time-dependent slip are directly inferred from the temporal modes of the viscoelastic Green's functions and the observed postseismic displacement. The IDAV method of solving the equation is straightforward in that only one temporal mode is used to decouple the viscoelastic Green's functions. This assumption is consistent with the analytical and numerical simulations of viscoelastic Green's functions for various geometries, and the simple assumptions of rheology and viscosity considered in this chapter.

We have examined the utility of the IDAV method using postseismic displacement observations made following the Dusky Sound earthquake and two simulated earthquakes, analytically and numerically. The results of inverting the postseismic displacements after the Dusky Sound earthquake imply viscosity values equal to or higher than $2 \times 10^{18}$ $\mathrm{Pa} \cdot \mathrm{s}$ for the viscoelastic half-space. The inversion method is applicable to more complex faulting scenarios using numerical simulation of the Green's functions, enabling consideration of realistic elastic-slab geometries for subduction earthquakes and lateral heterogeneity of elastic or viscoelastic properties. 


\section{Chapter 5}

\section{Observations and modelling of postseismic displace- ment during and following the 2010-2012 Canter- bury earthquake sequence}

\section{Abstract}

The 2010-2012 Canterbury earthquake sequence commenced with the 4 September $2010 \mathrm{M}_{\mathrm{W}}$ 7.1 Darfield and produced several destructive aftershocks, of which the most damaging, the 22 February $2011 \mathrm{M}_{\mathrm{W}} 6.2$ Christchurch earthquake, caused 185 fatalies and extensive damage to infrastructure. The sequence also produced postseismic deformation signals, which we analyse here using Interferometric Synthetic Aperture Radar (InSAR) and campaign Global Positioning System (GPS) data. Measurements made with ascending and descending acquisitions by the COSMO-SkyMed satellite suggest that poroelastic deformation induced by the Darfield earthquake terminated within one month, whereas longer-term dilatancy recovery continued for at least five months. Afterslip does not appear to have caused significant postseismic deformation after the Darfield earthquake. TerraSAR-X and campaign GPS measurements spanning the eastern end of the Greendale East Fault, which exhibited the greatest slip in the Darfield earthquake, reveal a pronounced deformation gradient and relative lineof-sight motion of $4 \mathrm{~mm} / \mathrm{yr}$ across the fault following the Christchurch earthquake. This $\sim 15 \mathrm{~km}$-long feature coincides with a concentration of aftershocks defining a previously recognised lineament, and we interpret the postseismic signal to represent afterslip on the lineament which is a continuation of the Western Christchurch Fault and subsidiary faults. 


\subsection{Introduction}

The 2010-2012 Canterbury earthquake sequence commenced with the $\mathrm{M}_{\mathrm{W}} \sim 7.1$ Darfield earthquake on 4 September 2010; the ensuing aftershock sequence included the $\mathrm{M}_{\mathrm{W}} \sim 6.2$ Christchurch earthquake on 22 February 2011, which caused 185 fatalities, and several other damaging aftershocks.

The Darfield and Christchurch earthquakes both occurred on previously unrecognised fault planes (Figure 5.1; Gledhill et al., 2011; Kaiser et al., 2012; Quigley et al., 2012), but subsequent seismological and geodetic studies have revealed complex fault structures. The Darfield earthquake produced a $\sim 30 \mathrm{~km}$-long surface rupture extending along the newly named Greendale Fault. Based on campaign Global Positioning System (GPS) measurements and airborne Light Detecting And Ranging (LiDAR) data, Quigley et al. (2012) estimated a static stress drop for the Darfield earthquake of $\sim 13$ $\mathrm{MPa}$. Regional and teleseismic moment tensor analysis conducted immediately after the Darfield earthquake revealed that reverse faulting occurred at the hypocentre but that most of the slip occurred on an east-west-striking vertical fault as dextral strikeslip motion (Gledhill et al., 2010, 2011). The state of stress in the crust encompassing the Canterbury earthquake sequence has been the focus of several studies (Sibson et al., 2011; Townend et al., 2012; Holt et al., 2013; Herman et al., 2014). The study of the contemporary regional stress field in the Canterbury region shows that the maximum horizontal compressive stress axis has a trend of $\sim 115^{\circ}$, similar to the orientation seen throughout much of the South Island (Holt et al., 2013; Townend et al., 2012).

Beavan et al. (2010b) obtained a preliminary geodetic source model for the Darfield earthquake based on the analysis of ground displacement measurements made with GPS and Interferometric Synthetic Aperture Radar (InSAR) data. Their source model revealed a complex rupture involving slip on several fault segments. The preferred model required a combination of reverse faulting on the Charing Cross fault, strike-slip faulting on the Greendale Fault, and normal faulting on the north-western segment as well as failure of the Hororata thrust Fault (Figure 5.1b). Using regional seismicity data, Gledhill et al. (2011) later refined the complex source structure in terms of aftershock hypocentres.

Elliott et al. (2012) modelled the geometry of the Darfield earthquake with seven fault segments using InSAR measurements, SAR amplitude offsets, a LiDAR DEM, aerial photogrammetry and satellite imagery along with teleseismic body-wave analysis. Their results agreed with the rupture initiated on a reverse fault and continued onto strike-slip fault segments. Syracuse et al. (2012) observed a NNW-SSE-striking 
band of seismicity corresponding to the seventh fault-segment identified from geodetic observations by Elliott et al. (2012).

Herman et al. (2014) calculated best-fitting double couple moment tensors and source depths for earthquakes having $\mathrm{M}_{\mathrm{W}}$ larger than 3.7 in order to determine the stress evolution throughout the Canterbury earthquake sequence. The focal mechanisms indicated major right-lateral slip on ENE fault planes and reverse faulting.

Shortly after the Christchurch earthquake, Beavan et al. (2011) inverted GPS and InSAR data to obtain models of the fault geometry and slip distribution. They explained most of the observed ground displacement by coseismic slip on two sub-parallel fault planes. In a subsequent study, Beavan et al. (2012a) revised the original source models for the Darfield and Christchurch earthquakes, and two significant events in June and December 2011. The results of their study reinforced earlier findings regarding the locations and geometries of the faults that had ruptured in each event, but added a left-lateral NNW-striking fault extending from the epicentre to the Darfield source model and two further faults (the strike-slip Western ENE fault and the reverse NNE fault) to the Christchurch source model (Figure 5.1b).

The Christchurch earthquake was notable for the high ground accelerations it produced near the epicentre. Fry et al. (2011) documented peak ground accelerations of $2 \mathrm{~g}$ and reported that asymmetry in the vertical-component seismograms was consistent with non-linear behaviour of near-surface materials. Kinematic source modelling of the Christchurch earthquake indicated maximum slip of $\sim 4 \mathrm{~m}$ at a depth of $\sim 4 \mathrm{~km}$ and a rupture velocity of $2.8 \mathrm{~km} / \mathrm{s}$ (Holden et al., 2011).

Barnhart et al. (2011) computed static stress changes induced by the Darfield earthquake and concluded that the Christchurch earthquake nucleated in a positive region of static Coulomb stress changes, with a peak of $0.31 \mathrm{MPa}$. In contrast, the Coulomb stress changes calculated by Beavan et al. (2011) in the vicinity of the Christchurch event are very small, although the region is still located in the positive Coulomb stress change area. The difference between the magnitude of the Coulomb stress changes can be related to the different source models that have been used for the Darfield and Christchurch events in the studies by Beavan et al. (2011) and Barnhart et al. (2011).

Bannister and Gledhill (2012) summarised the evolution of the Canterbury earthquake sequence as well as reverse and strike-slip aftershocks. This and other studies have identified a lineament defined by the concentration of the aftershocks extending from further south of the Greendale East Fault to the Western Fault that ruptured during the Christchurch earthquake (Figure 5.1b; Sibson et al., 2011; Syracuse et al., 2012; Herman 
et al., 2014) . Sibson et al. (2011) inferred a step-over, a potential dilational zone, throughout the area between the Greendale East Fault and the lineament. Dilational zones are known as kinematic barriers to arrest the rupture and dissipate the terminated strain energy by slip transfer to the nearby fault segments (Sibson, 1986).

Motagh et al. (2014b) undertook the first study of postseismic displacement following the Darfield earthquake using differential interferometry applied to two months' observations. They constructed a displacement time series based on ascending COSMOSkyMed (CSK) acquisitions spanning the interval between September 2010 and February 2011. The early postseismic displacement field reveals motions towards the ascending CSK satellite along the Greendale East Fault and away from the satellite along the Charing Cross Fault and within the step-over between the Greendale East and Central Faults.

This study examines the postseismic ground displacement following the Darfield and the smaller but more destructive Christchurch earthquakes. Following the Darfield earthquake and prior to the Christchurch earthquake, we focus specifically on the area extending from near the Hororata Fault to the easternmost end of the Greendale East Fault (Figure 5.1b and c). The area encloses the faults related to the Darfield earthquake. Following the Christchurch earthquake, the area of interest spans the region between the easternmost end of the Greendale East Fault and the urban area of Christchurch. This area encompasses the faults that ruptured during the Christchurch earthquake and those that ruptured subsequently during the 13 June 2011 and 23 December 2011 events (Figure 5.1b).

In previous studies of postseismic displacement following large earthquakes, several processes yielding different spatiotemporal patterns of surface deformation have been proposed.

- Viscoelastic relaxation, transferring stress from the ductile lower crust and upper mantle to the brittle upper crust (e.g. Freed and Lin, 1998, 2001; Rydelek and Sacks, 2001; Freed, 2005; Pollitz et al., 2006a; Freed et al., 2007; Tabrez et al., 2008; He et al., 2011; Decriem and Árnadóttir, 2012);

- Poroelastic rebound, in which instantaneous changes in confining stress induced coseismically cause pore pressure changes which equilibrate postseismically (Peltzer et al., 1996, 1998);

- Rate-strengthening afterslip on the faults that slipped coseismically (Lapusta et al., 2000; Rice et al., 2001; Barbot et al., 2009), as formulated in terms of a rate-and-state-dependent friction relationship (Ruina, 1983; Dieterich, 1994);

- Inverse afterslip model, meaning afterslip occurring in both the upper and lower 
crust on the mainshock fault surface (e.g. Savage and Church, 1974; Johnson et al., 2012; Wen et al., 2012a; Reddy et al., 2013); and

- Dilatancy recovery, by which the crust recovers its preseismic strength (Fielding et al., 2009) and which has been suggested to have occurred following the Darfield earthquake by Reyners et al. 2014 (see Section 2.2.5).

The main objective of this chapter is to understand which of the postseismic mechanisms above can best account for ground displacements observed following the Darfield and Christchurch earthquakes. For the Darfield earthquake, we use InSAR observations acquired within one month of the earthquake and approximately five months of displacement time series from October 2010 to February 2011 using the Italian CSK satellite constellation. For the Christchurch event, we use campaign GPS velocities acquired annually in early 2012 to 2015 and InSAR time-series analysis derived from TerraSAR-X (TSX) acquisitions.

\subsection{Methodology}

\subsubsection{Observations}

\section{GPS velocities}

We analyse campaign GPS data acquired annually between early 2012 to 2015 in the Canterbury region ${ }^{1}$. We solve for station coordinates, satellite orbit and Earth rotation parameters, and estimate atmospheric zenith delay every two hours and three atmospheric gradients per day. We use the IGS08 (International GNSS Service; Schmid et al., 2007) azimuth and elevation-dependent absolute phase centre model with an elevation cutoff angle of $10^{\circ}$ for the ground-based antennas and apply the FES2004 (Finite Element Solutions (FES) tidal atlases; Lyard et al., 2006) ocean loading model. The positions are transformed into the ITRF08 (International Terrestrial Reference Frame; version 2008) Pacific plate fixed reference frame (Altamimi et al., 2012), via a sixparameter transformation (translations and rotations). We minimise the residual of 30 reference stations in the IGS network (Herring et al., 2008) and subtract the velocity of site MQZG in three successive years (Figure 5.2).

\footnotetext{
${ }^{1}$ Processed by Dr. Sigrún Hreinsdóttir
} 


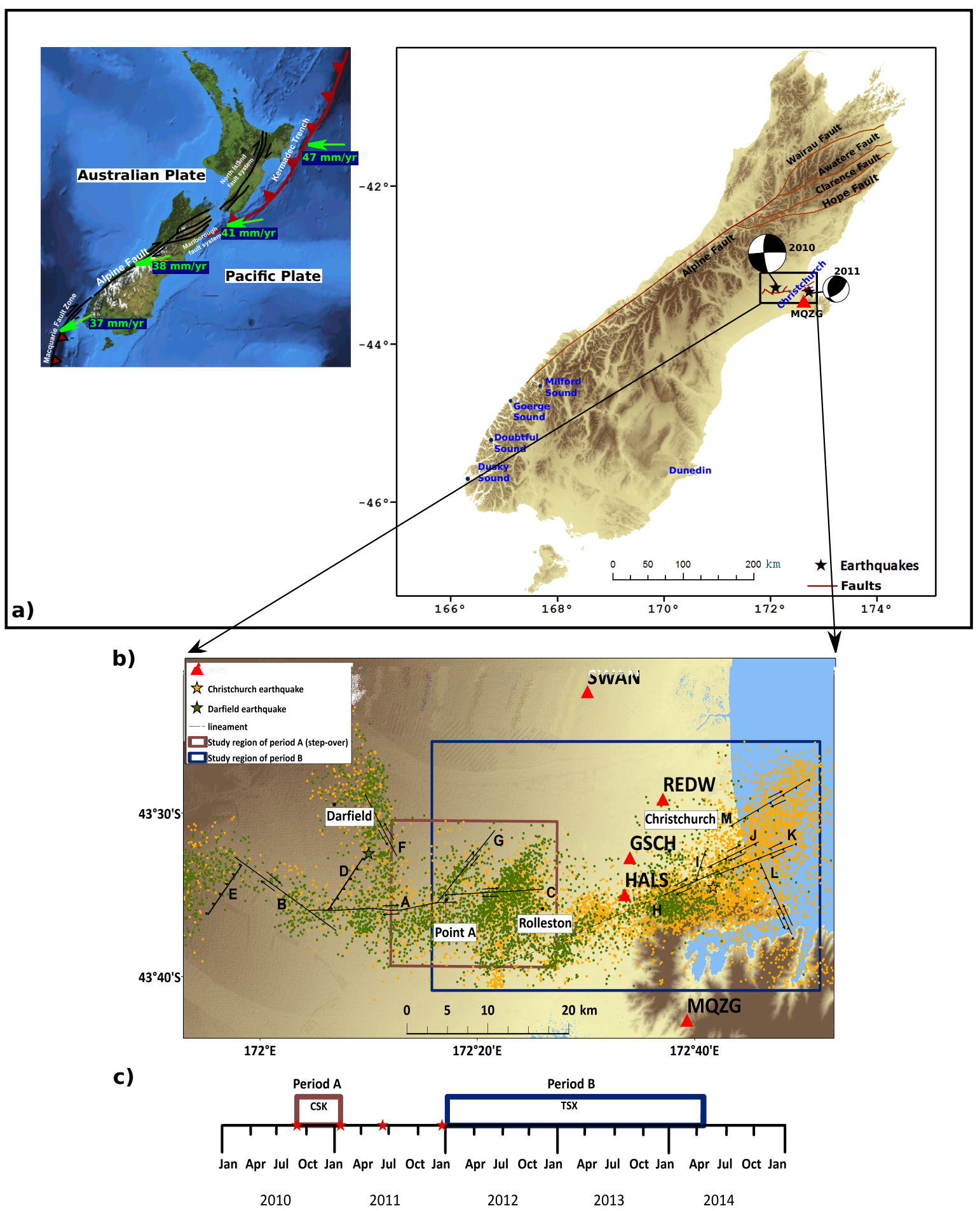

Figure 5.1: a) Tectonic setting of the South Island, New Zealand and focal mechanisms of recent earthquakes, namely the 2010 Darfield and 2011 Christchurch earthquakes (continued on the next page).

\section{Interferometric Synthetic Aperture Radar (InSAR) analysis}

To map and interpret postseismic displacements following the Darfield earthquake, we use interferograms acquired by the CSK satellite (Figure 5.3a and b) and generated by Motagh et al. (2014b). The interferograms enclose the principal fault segments 
Figure 5.1: b) Map of the study area showing the epicentres of the Darfield (Canterbury) earthquake and the Christchurch earthquake (green and yellow stars, respectively). Also shown are the surface traces of faults inferred to have ruptured during the Darfield and Christchurch earthquakes and large aftershocks: A) Greendale Central, B) Greendale NWSE, C) Greendale East, D) Charing Cross, E) near Hororata, F) NNW from the epicentre, G) NE from step-over Faults related to the Darfield earthquake, H) Western, I) NNE, J) Eastern Christchurch Faults related to the Christchurch earthquake, K) SW-NE, L) NW-SE Faults related to the 13 June 2011 event and M) SW-NE Fault related to the 23 December 2011 event (Beavan et al., 2012a). Green dots indicate the aftershocks following the Darfield and prior to the Christchurch earthquake. Yellow dots indicate aftershocks following the Christchurch earthquake. Red triangles indicate cGPS sites. c) Time intervals related to the ascending CSK and descending TSX interferograms in period A (first interval) and B (second interval). Red stars indicate the Darfield, Christchurch, 13 June and 23 December 2011 earthquakes. Aftershocks are reported by http://quakesearch.geonet.org.nz/.

Table 5.1: Incidence angle and temporal baselines of interferograms in Figure 5.3 and 5.5.

\begin{tabular}{c|c|c|c} 
Data type & Image date & Incidence $^{\text {angle }}{ }^{\circ}$ & $B_{\text {temp }}$ (days) \\
\hline CSK ascending & $20100906-20100922$ & 22 & 17 \\
CSK descending & $20100913-20100929$ & 47 & 17 \\
TSX descending & $20100907-20100918$ & 40 & 12
\end{tabular}

that ruptured during the Darfield earthquake. In addition, we use 12 ascending CSK images to generate a displacement time series between October 2010 and February 2011 (Figure 5.4). We present temporal displacement results for the step-over between the Greendale East and Central Faults. The Small BAseline Subset (SBAS) network comprises 38 interferograms in which pixels with coherence above a threshold of 0.2 are selected. After 3D phase unwrapping, the time series of displacement was estimated using StaMPS (Hooper and Zebker, 2007; Hooper, 2008; Hooper et al., 2010).

An additional post-Darfield interferogram is generated from two descending TSX acquisitions that in conjunction with the descending CSK interferogram help to constrain the sense of motion along the easternmost end of the Greendale East Fault (Figure 5.5). We generate the interferogram using standard techniques implemented in the commercial software SARscape. We do not model either the descending CSK interferogram (Figure 5.3b) or the TSX interferogram directly (Figure 5.5), because of the strong long-wavelength orbital and tropospheric errors (Motagh et al., 2014b). Table 5.1 presents incidence angle and temporal baselines of each interferogram in Figure 5.3 and 5.5 .

Following the Christchurch earthquake, we use 66 TSX acquisitions in descending orbits with an incidence angle of $40^{\circ}$ and heading angle of $196^{\circ}$ acquired between March 2011 and May 2014. After reading and cropping the Single Look Complex (SLC) products, satellite orbits for all images are extracted and Digital Elevation Model (DEM) 


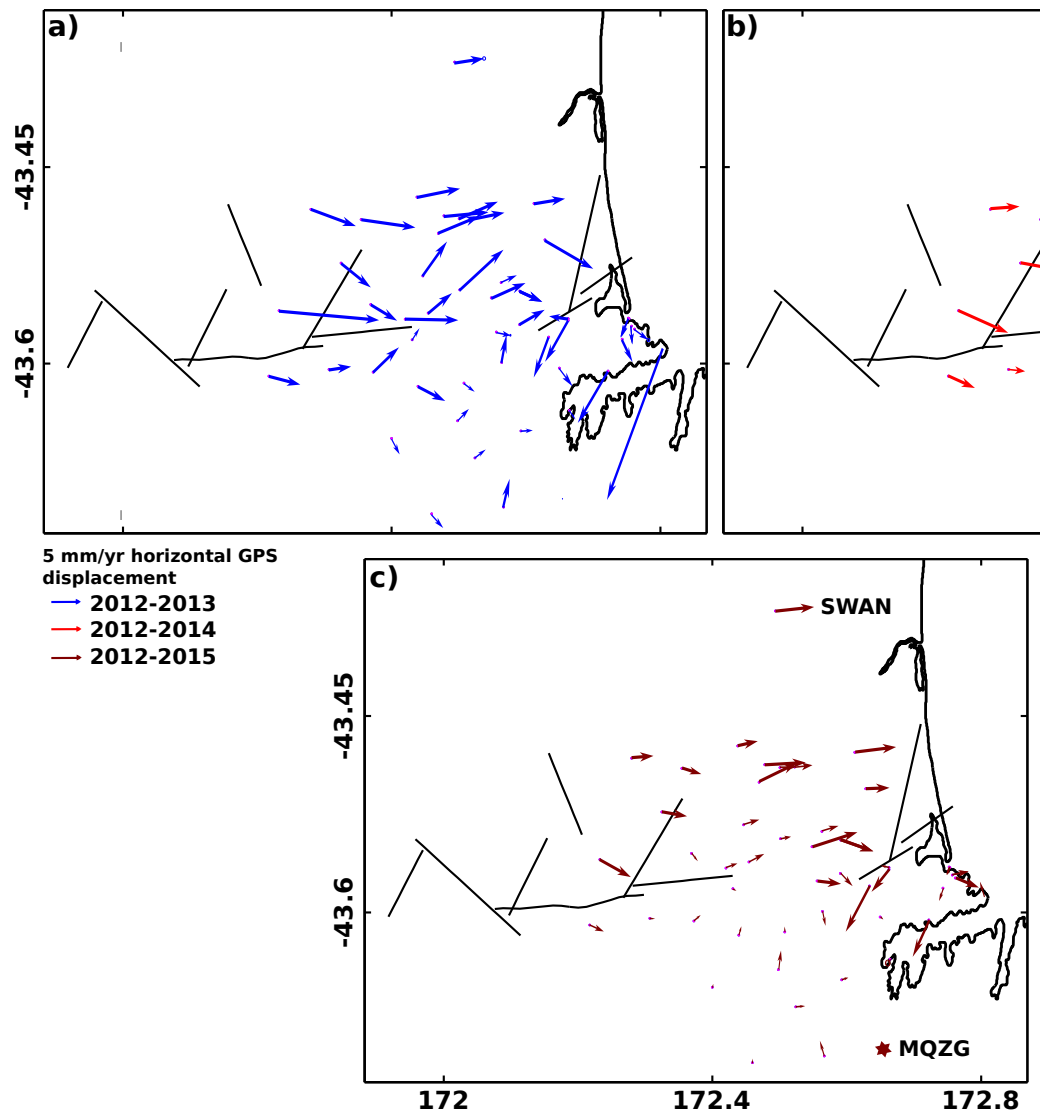

Figure 5.2: Arrows represent GPS horizontal velocities in the Pacific plate reference frame relative to the continious GPS site MQZG for three periods: a) 2012-2013 (blue), b) 2012-2014 (red) and c) 2012-2015 (maroon). 


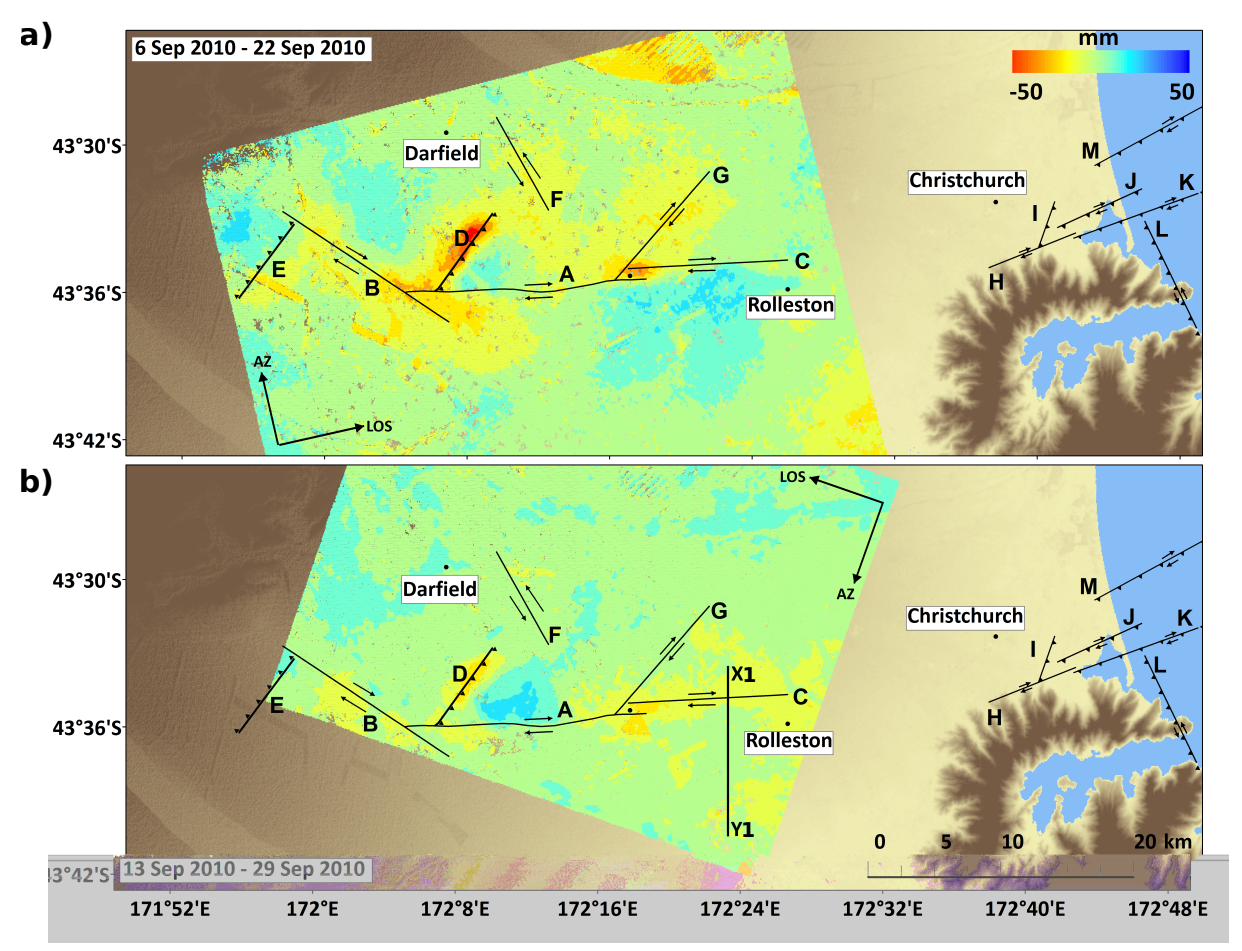

Figure 5.3: a) Unwrapped interferogram acquired from ascending CSK orbits between the $6^{\text {th }}$ and $22^{\text {nd }}$ of September 2010. b) Unwrapped interferogram acquired from descending CSK orbits between the 13th and 29th of September 2010. Red colours indicate movement away from satellite and blue colours indicate movement towards the satellite.

offset corrections applied using the LINZ (Land Information New Zealand) $40 \mathrm{~m}$ digital elevation model. All pairs for each dataset are coregistered and resampled and 65 master-slave interferograms are produced using the software package DORIS (Kampes and Usai, 1999). The TSX acquisition from 20 July 2012 was selected as the master image for the postseismic time interval between 2 March 2011 and 22 May 2014 as it yields the minimum temporal and perpendicular baselines for the master-slave pairs. Time series analysis with the default StaMPs parameters is only conducted for Slowly Decorrelating Filtered Phase (SDFP) pixels (Hooper and Zebker, 2007; Hooper, 2008; Hooper et al., 2010). The maximum density of the candidate pixels is 2 percent (per $\mathrm{km}^{2}$ ). The effect of turbulent atmospheric delay has been mitigated by stacking numerous acquisitions. The stratified atmospheric delay masks the displacement signal over the area on the south-eastern corner of the case study. For this reason we discard measurements from the region with elevations above $150 \mathrm{~m}$ in the final velocity calculations.

The mean Line Of Sight (LOS) velocity for data acquired between March 2011 and May 2014 is illustrated in Figure 5.6a: this velocity includes coseismic offsets associated with the June and December 2011 earthquakes (see Appendix D), as well as postseismic displacements. Due to the effects of the June and December 2011 events, we also solve 


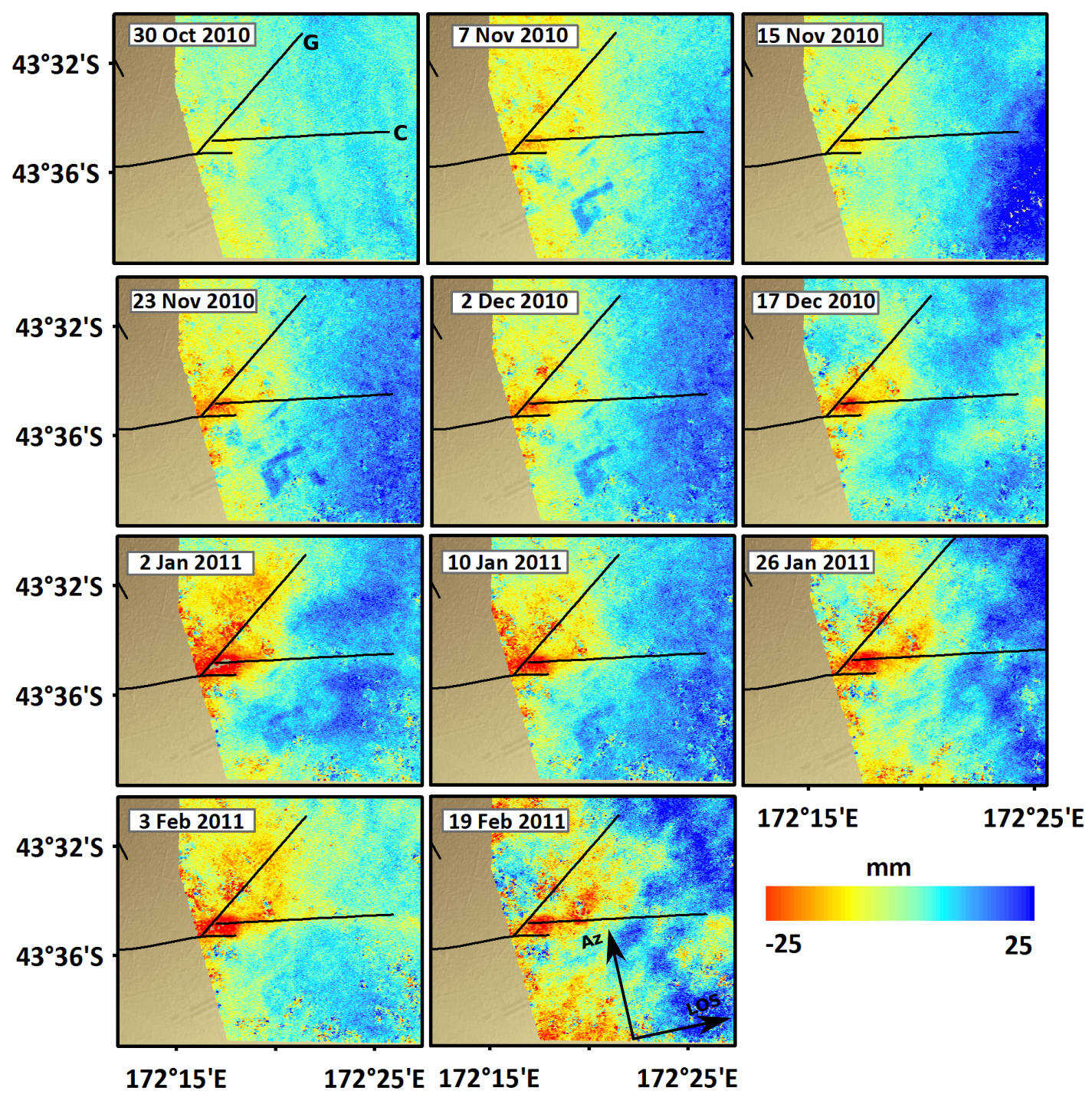

Figure 5.4: Observed time series of postseismic displacement acquired from ascending CSK acquisitions following the Darfield and prior to the Christchurch earthquakes on the Greendale step-over between October 2010 and February 2011 relative to 14 October 2010. Red colours indicate movement away from satellite and blue colours indicate movement towards the satellite.

for the mean LOS velocity using data acquired between January 2012 and May 2014 only (Figure 5.6b; the standard deviations of the velocity results are presented in Appendix D).

\subsubsection{Analytical and numerical models}

In this study, we simultaneously invert the ground displacement after subtracting poroelastic effects to estimate the dilatancy recovery and afterslip responses. Methods of forward modelling viscoelastic, poroelastic, and afterslip deformation are reasonably 


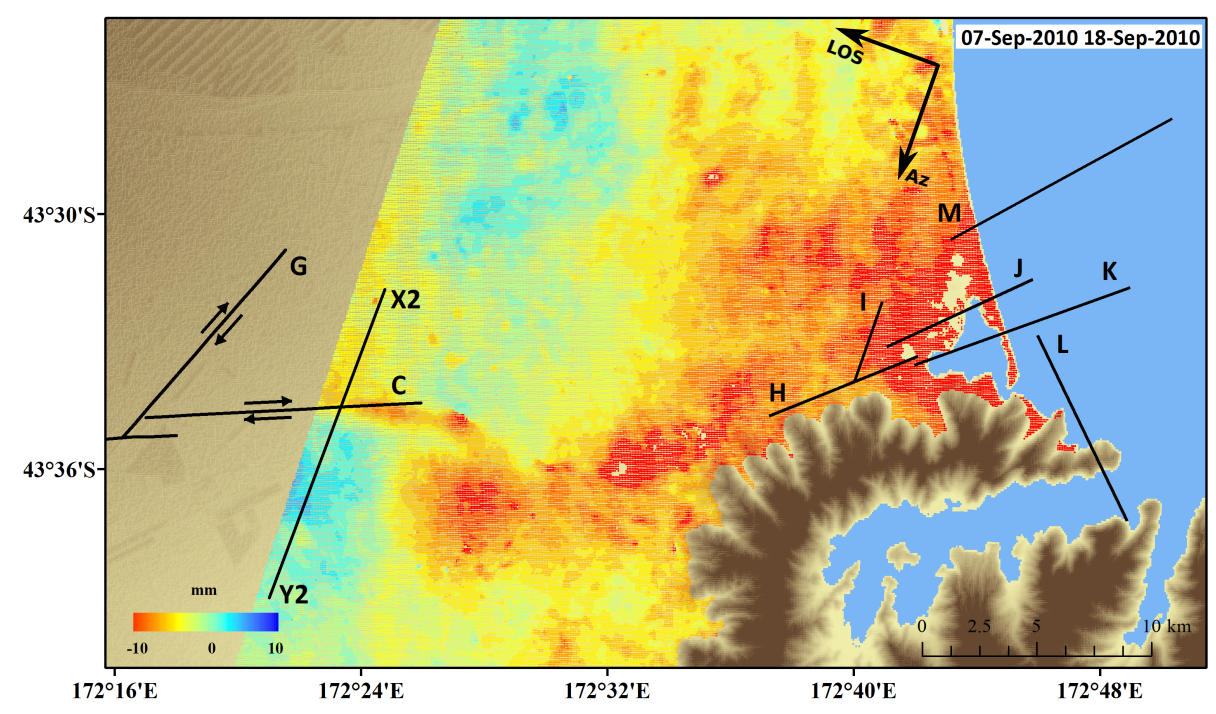

Figure 5.5: Unwrapped interferogram acquired from descending TSX orbits between the $7^{\text {th }}$ and $18^{\text {th }}$ of September 2010 within one month of the Darfield earthquake. Red colours indicate movement away from satellite and blue colours indicate movement towards the satellite.

standard and are not discussed in detail here.

We model viscoelastic relaxation, poroelastic rebound and frictional afterslip with the numerical code PyLith (Aagaard et al., 2009, 2013, 2015), Fortran codes of Wang and Kümpel (2003) and RELAX (Barbot et al., 2009; Barbot and Fialko, 2010a), respectively.

\section{Simultaneous poroelastic rebound, dilatancy recovery and afterslip responses}

To combine poroelastic rebound, dilatancy recovery and afterslip, we first subtract a forward estimate of poroelastic rebound from the observations. We then invert the residual observations to estimate the components of slip (afterslip) and volumetric changes (dilatancy recovery) occurring on assumed faults. Appropriate constraints must be imposed in order to ensure that the sense of motion represented by the resulting afterslip model is consistent with that expected for each fault.

One way of accounting for dilatancy recovery is to assume a regular grid of Mogi point sources on the fault planes that ruptured coseismically (Mogi, 1958). This approach is simplistic in that it does not completely describe the physical process, as Fielding et al. (2009) explained. However, the advantage of assuming the same grid for the Mogi point sources as the slip distribution is that the only parameter we estimate is the volume change at each point source with defined coordinates and depth. In this study, therefore, we simultaneously solve for afterslip consisting of strike- and dip-slip 


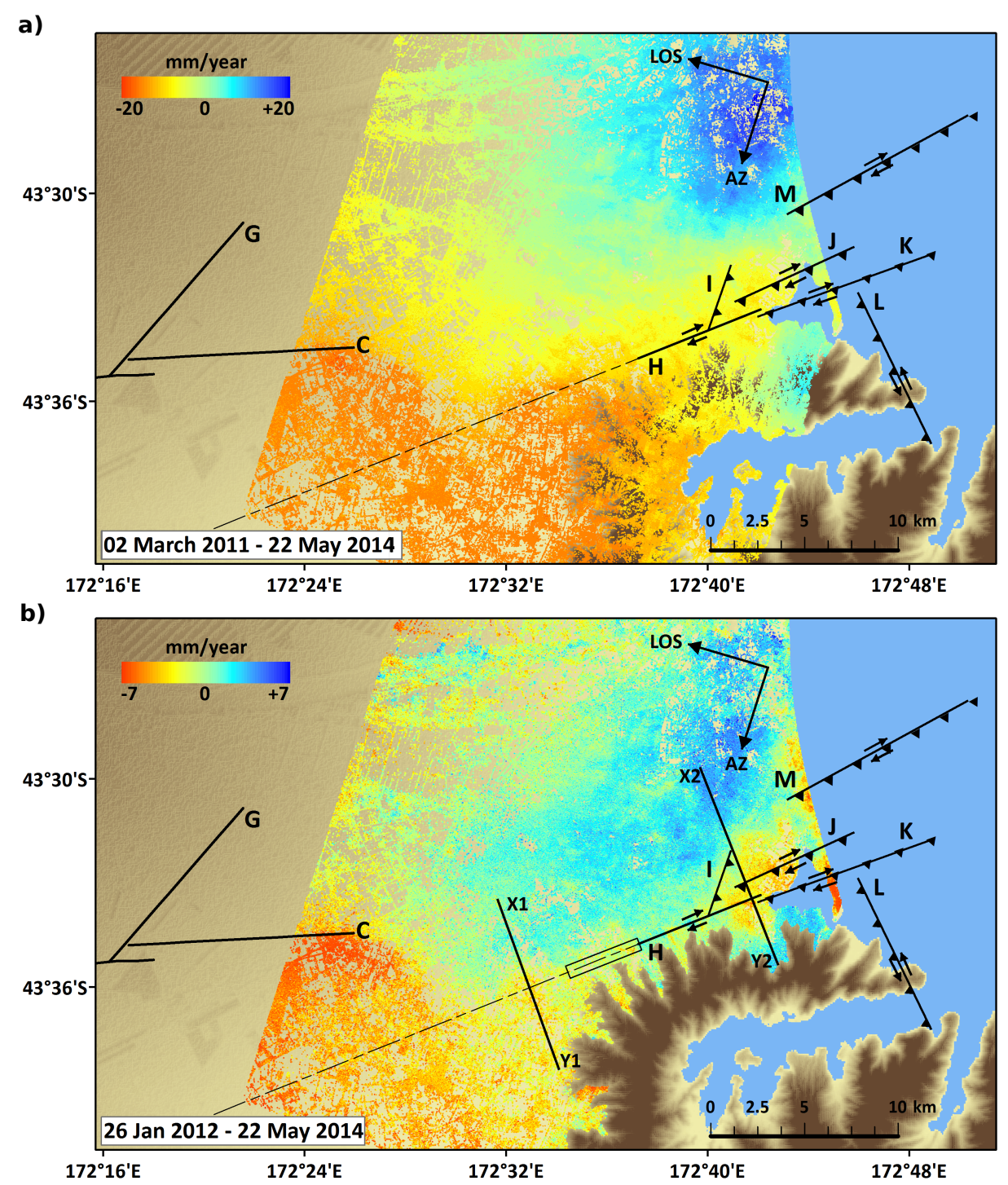

Figure 5.6: a) Observed mean LOS velocity acquired from TSX acquisitions following the Christchurch earthquake between March 2011 and May 2014. b) Observed mean LOS velocity acquired from TSX acquisitions following the Christchurch earthquake between January 2012 and May 2014. Red colours indicate movement away from the satellite and blue colours indicate movement towards the satellite. Dashed line is micro-earthquake lineament (extension of $\mathrm{H})$. Other faults are described in Figure 5.1b.

components $\left(s_{s}\right.$ and $\left.s_{d}\right)$ and volume change $(\Delta v)$ at each point of the defined regular grid.

We can express the observation equation relating the observed ground displacement $(d)$ and the parameters of interest $(s)$ as follows

$$
d=G s+\epsilon=\left[\begin{array}{ll}
G_{f} & G_{v}
\end{array}\right]\left[\begin{array}{c}
s_{s} \\
s_{d} \\
\Delta v
\end{array}\right]+\epsilon
$$

where $G$ is the combined (augmented) design matrix, $G_{f}$ and $G_{v}$ are functions of the dislocation model and the Mogi point source parameters (Okada, 1985; Mogi, 1958), 
and $\epsilon$ is the residual.

If $X, Y$ and $D$ are the east, north coordinates and depth of the point source with respect to the ground location and $R=\sqrt{X^{2}+Y^{2}+D^{2}}$, then the three-dimensional ground displacement induced by a Mogi point source is given by

$$
d_{v}=\frac{\nu-1}{\pi R^{3}}\left[\begin{array}{l}
X \\
Y \\
D
\end{array}\right] \Delta v+\epsilon_{v}=G_{v} \Delta v+\epsilon_{v}
$$

where $\nu$ is Poisson's ratio. Since observations are made in the LOS direction, we project Equations 5.1 and 5.2 in the appropriate direction using the heading and incidence angles of the sensors.

In order to solve for the two components of afterslip on the fault planes as well as the volume changes, we use the linear least squares approach to invert the displacement observations. Equation 5.1 is still an ill-posed problem and requires spatial smoothing. During the inversion, the following function is minimised

$$
f=\|d-G s\|^{2}+\gamma^{-2}\left\|\nabla^{2} s\right\|^{2}
$$

$\nabla^{2}$ is the approximation of the Laplacian operator and $\gamma$ determines the trade-off between misfits and smoothness of parameters.

\subsection{Post-Darfield displacement}

\subsubsection{InSAR observations}

Our observations of displacement following the Darfield earthquake exhibit several notable features (Figure 5.3, 5.4, 5.5 and 5.7):

1. Localised movement away from the satellite of $\sim 20 \mathrm{~mm}$ within the Greendale step-over at the intersection between fault segments (A) and (C), within approximately one month of the Darfield earthquake, and cumulative movement away from the satellite of $\sim 25 \mathrm{~mm}$ between October 2010 and February 2011;

2. Movement away from the satellite on the Greendale NW-SE (B), Charing Cross (D), near Hororata (E), NNW from the epicentre (F) and NE from step-over (G) Faults;

3. Movement towards the satellite on the southern side of the Greendale East Fault (C) and within the region between the Greendale NW-SE (B) and Charing Cross 
Faults (D); and

4. Movement away from the satellite in the corner between the Greendale Central (A) and the Greendale NW-SE Faults (B).

The signals were originally noted by Motagh et al. (2014b) based on an analysis of a short-term record of ascending CSK acquisitions. Due to the incidence angle $22^{\circ}$ of the acquisitions, ground displacements projected in the LOS of the satellite are more sensitive to the vertical component of displacement than the horizontal components. The descending interferograms obtainable from descending CSK (Motagh et al., 2014b) and TSX observations are contaminated by long-wavelength tropospheric and orbital artefacts, which preclude an accurate interpretation of the sense of motion. Despite the existence of these long-wavelength artefacts, displacement profiles across the Greendale East Fault (Figure 5.7) from descending acquisitions of the CSK and TSX satellites both reveal motions towards the satellites on the southern side of the Greendale East Fault. Accordingly, we can interpret the observed signal as being mostly vertical motion rather than representing right-lateral displacement.

\subsubsection{Modelling results}

\section{First-order approximation}

We calculate the viscoelastic response of a Maxwell material to the coseismic stress changes due to the Darfield earthquake using the numerical code PyLith (Aagaard et al., 2009, 2013, 2015). An elastic thickness of $35 \mathrm{~km}$ is appropriate for the Canterbury region based on the 3-D seismic velocity model of New Zealand (Eberhart-Phillips et al., 2010). We assume a viscosity value of $10^{18} \mathrm{~Pa} \cdot \mathrm{s}$ for the viscoelastic half-space in order to maximise the effect of viscoelastic relaxation. The computed viscoelastic response in the time interval between the Darfield and Christchurch earthquakes is negligible $(<2 \mathrm{~mm}$ throughout the model) and is not discussed further below.

To understand what other physical processes produce the observed postseismic displacement, we also consider afterslip on faults that had ruptured coseismically. We use the public-domain software package RELAX, which implements the method described by Barbot et al. (2009), and assume the fault geometries and coseismic slip distributions computed by Beavan et al. (2012a). This approach allows us to compare the predicted three dimensional displacement field with the observations and examine whether an afterslip model alone can satisfy the observed postseismic displacement. We emphasise that the results of employing rate-strengthening afterslip, using RELAX, represent a 

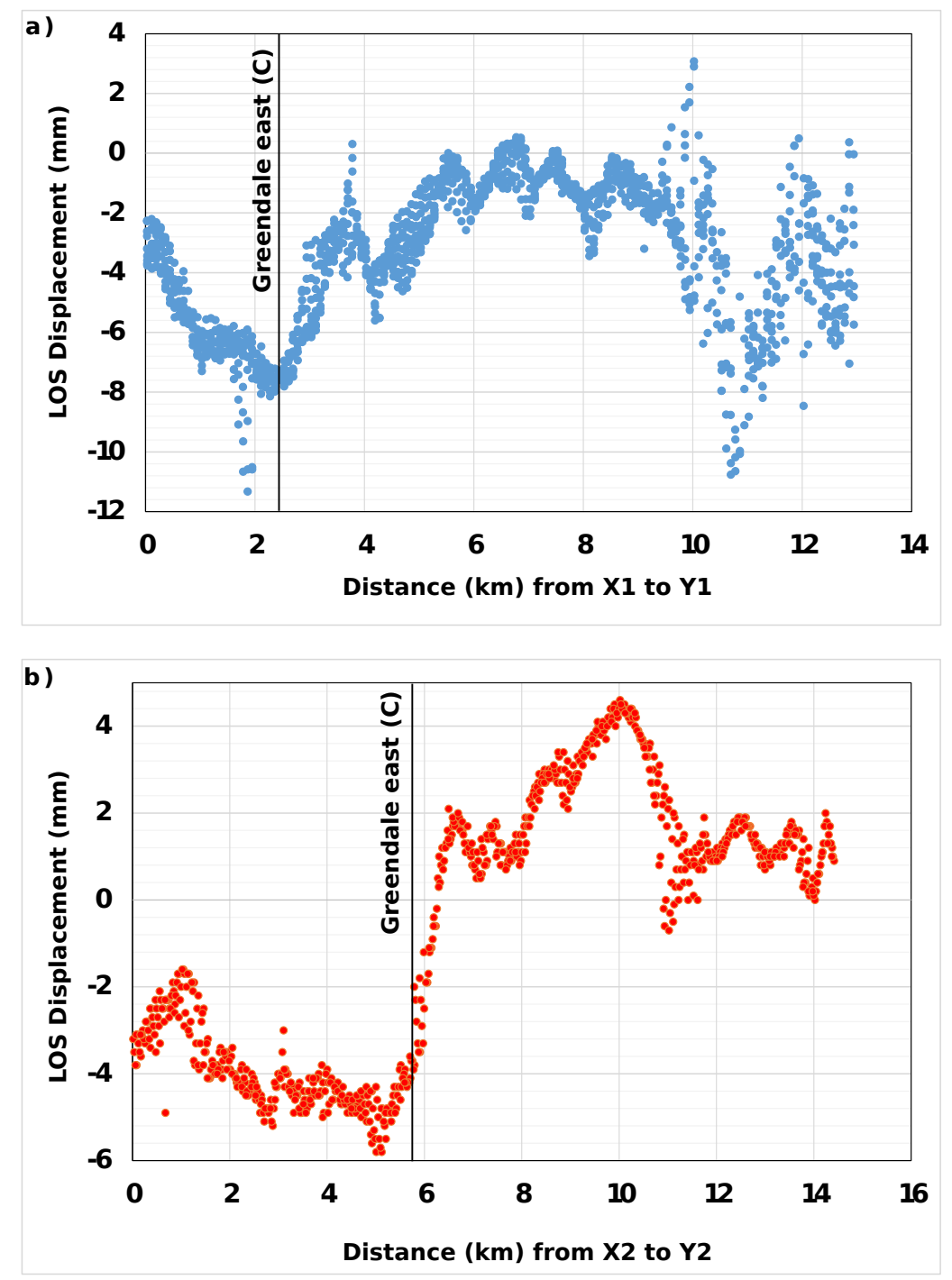

Figure 5.7: Profiles of displacement along the X1Y1 line shown in Figure 5.3b and X2Y2 in Figure 5.5. We can see movements towards the satellite of $\sim 8 \mathrm{~mm}$ along the X1Y1 line and along the $\mathrm{X} 2 \mathrm{Y} 2$ in Figure 5.3b and Figure 5.5, respectively. The signal is contaminated and reduced by a large amount of orbital and tropospheric error in the region.

first-order approximation of the sense of postseismic displacement due to afterslip on the assumed faults.

Assuming values of $50 \mathrm{~mm} / \mathrm{yr}$ and $5 \mathrm{MPa}$ for the two parameters governing ratestrengthening afterslip (rock and frictional properties: $\dot{s}_{0}$ and $a \sigma$; see Barbot et al., 2009) and constraining the afterslip rake angles using the corresponding average coseismic rake angles on each fault, we compute postseismic displacements after the Darfield earthquake. Note that we do not use a formal method to choose these parameters: we test a range of 10-100 mm/yr and 1-10 MPa for the two model parameters to simulate the postseismic displacement within the observed range.

The modelled east, north and vertical components of displacement and the projected 
displacement in the ascending LOS of the CSK satellite within approximately one month of the Darfield earthquake are illustrated in Figure 5.8. The movement away from the satellite observed in the step-over is not predicted by the model, nor is the signal in the corner between the Greendale Central (A) and the Greendale NW-SE Faults (B). The east component of displacement does exhibit movement towards the satellite on the southern side of the Greendale East Fault (C), but the LOS component mainly reflects the vertical displacement and is only weakly influenced by the east component. In contrast, the movement away from the satellite along the blind Charing Cross Fault (D) is represented reasonably well by the afterslip model. Movement towards the satellite is observed in the ascending CSK interferogram (Figure 5.3) between the northern part of the Greendale NW-SE Fault and the western side of the Charing Cross Fault. This motion cannot be accounted for with the assumed rate-strengthening afterslip rheology. Therefore, the afterslip model with the assumed parameters does not fit the overall sense of the observations well, with the exception of the signal associated with the Charing Cross Fault.

The forward model of rate-strengthening afterslip described above requires knowledge of two parameters $\left(\dot{s}_{0}\right.$ and $\left.a \sigma\right)$. We have also investigated afterslip models estimated using inversion techniques by Motagh et al. (2014b) to examine whether these provide better matches to the observations (Figure 5.9). We observe the following points suggesting that afterslip does not adequately account for the observations:

- Significant reverse-slip on the Greendale NW-SE Fault, whereas the coseismic slip suggested by Beavan et al. (2012a) is mainly of right-lateral and normal sense (Figure 5.9a);

- Significant normal-slip on the right-lateral Greendale Central (Figure 5.9c);

- Significant reverse-slip on the right-lateral NE of the step-over and on the rightlateral Greendale East Faults (Figure 5.9d and 5.9e).

On this basis, we infer that postseismic mechanisms other than afterslip is responsible for producing the short-term post-Darfield ground displacements presented here and in the studies by Beavan et al. (2012a) and Motagh et al. (2014b). We next consider whether poroelastic rebound can better account for the sense and spatial extent of postseismic displacement.

\section{Combined poroelastic rebound and dilatancy recovery}

We estimate the displacement induced by poroelastic rebound following the Darfield earthquake using the method of Wang and Kümpel (2003). We use the national New 

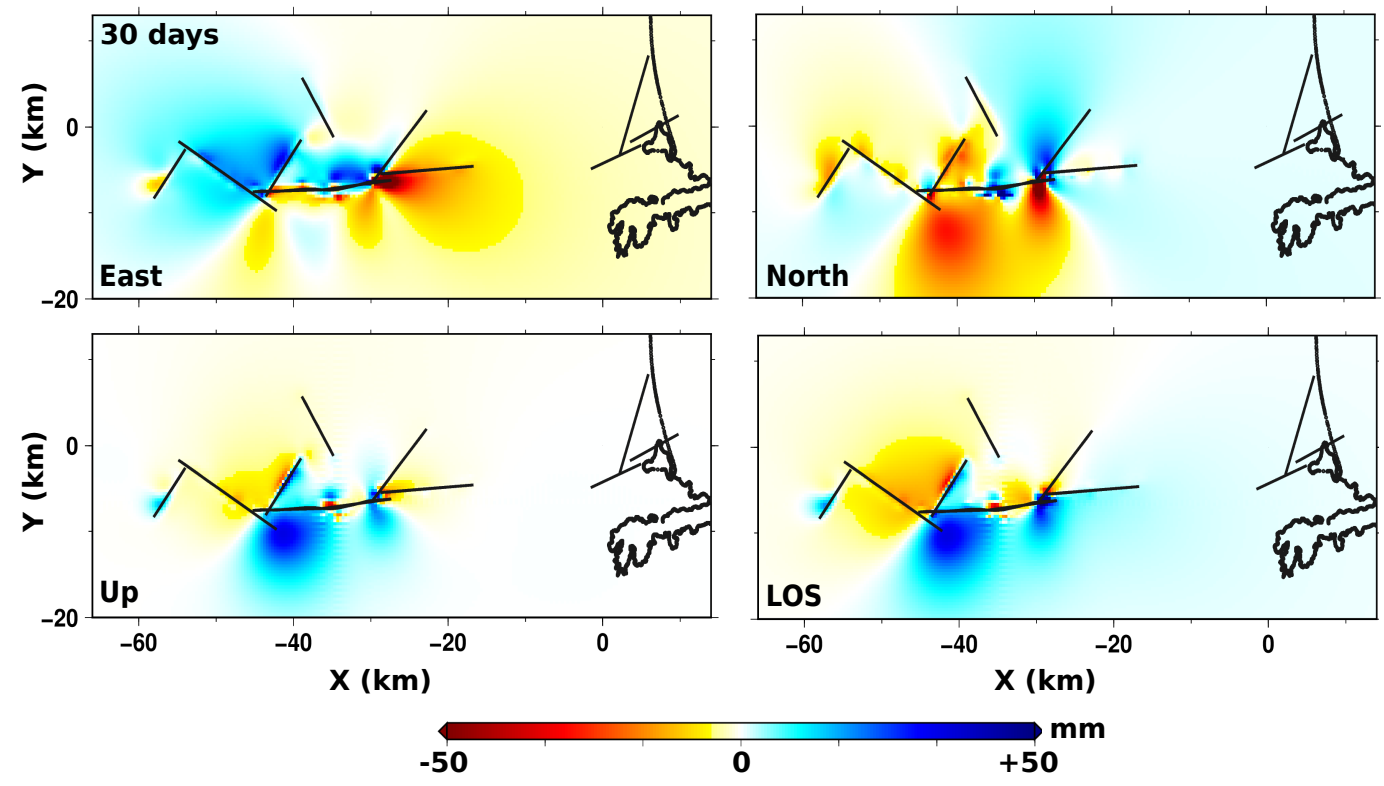

Figure 5.8: Surface displacement projected in the LOS of ascending CSK, one month after the Darfield earthquake based on a model of rate-strengthening afterslip on the previously ruptured faults during the Darfield earthquake. Red colours indicate movement away from satellite and blue colours indicate movement towards the satellite. Other faults are described in Figure 5.1b.

Zealand velocity model to assign the P- and S-wave velocities (Eberhart-Phillips et al., 2010) of between two and nine layers. The two-layer representation is a simplified version of the nine-layer model that is directly inferred from the national velocity model. Values for hydraulic diffusivity of either 0.1 or $1.0 \mathrm{~m}^{2} / \mathrm{s}$ above a specified depth and $0.0001 \mathrm{~m}^{2} / \mathrm{s}$ below that depth have been considered on the basis of the assumed shallow extent of poroelastic processes. The interface depths at which hydraulic diffusivity changes from higher to lower values are assigned values of either 1.0 or $1.75 \mathrm{~km}$ in our models. We assume values of 0.5 and 0.6 for Skempton's coefficient and 0.6 and 0.8 for Biot coefficient, and consider a range of model parameters in fitting the observations (Table 5.2).

Poroelastic rebound curves associated with various model parametrisations are illustrated in Figure 5.10 in the LOS direction of the ascending CSK orbit. The calculated displacement mainly represents the vertical component of movement due to the poroelastic rebound effects.

In order to best account for the observations illustrated in Figure 5.3, a high hydraulic diffusivity value of $1 \mathrm{~m}^{2} / \mathrm{s}$ is required. Cases with higher values of 0.6 and 0.8 for Skempton's and Biot coefficients, respectively, overestimate the observed range increase and range decrease patches assuming hydraulic diffusivity of $1 \mathrm{~m}^{2} / \mathrm{s}$ (Figure 5.10e). In contrast, a lower hydraulic diffusivity value of $0.1 \mathrm{~m}^{2} / \mathrm{s}$ with the same 

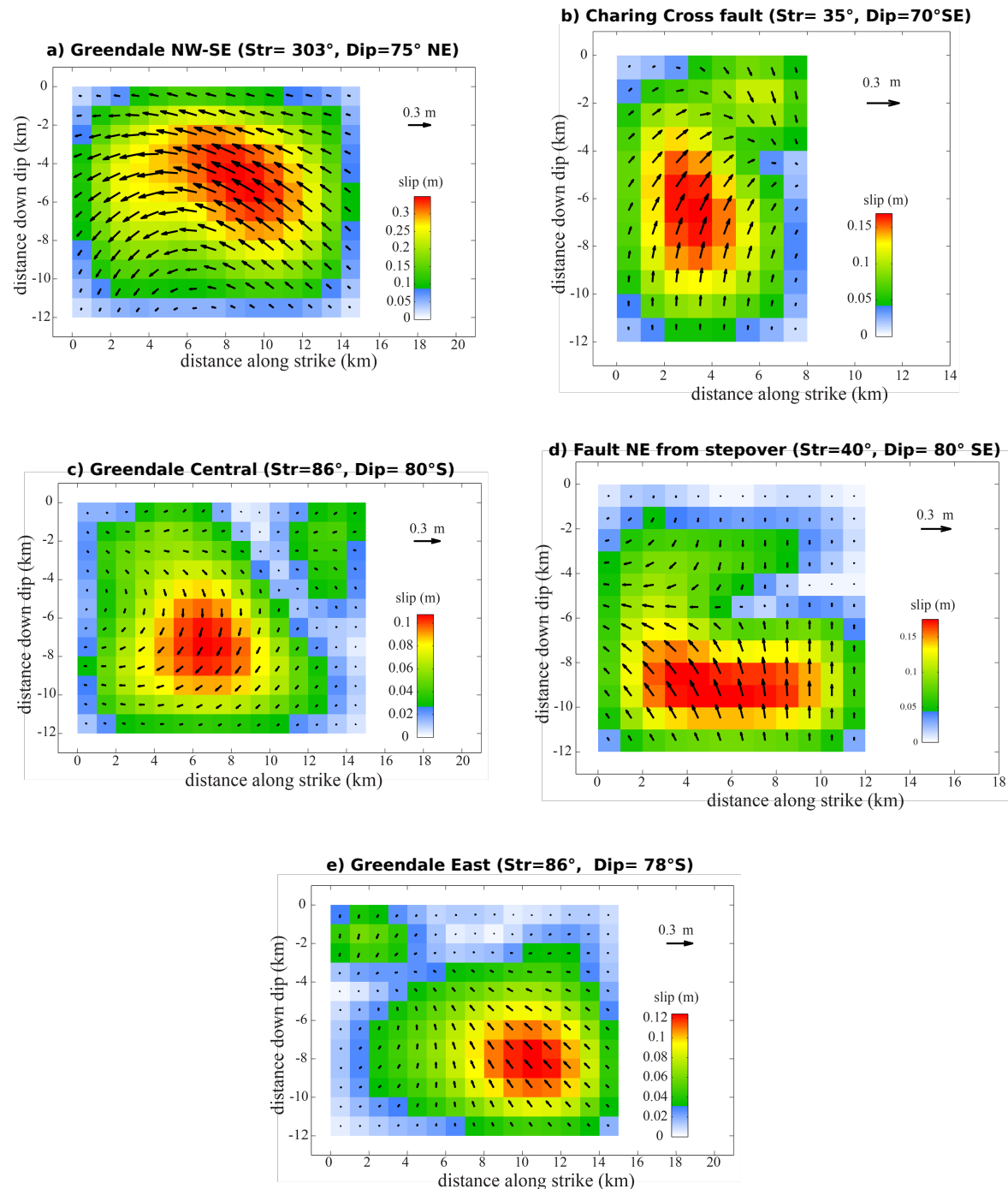

Figure 5.9: Modeling results for postseismic afterslip (version I, January 2012; Motagh et al., 2014a): Other faults are described in Figure 5.1b.

poroelastic coefficients as in Model 12 poorly accounts for the observed postseismic features (Figure 5.10f).

Our preferred poroelastic model is the two-layer Model 5, which has a Root Mean Square Error (RMSE) of $9 \mathrm{~mm}$, Skempton's and Biot coefficients 0.5 and 0.6, respectively, and diffusivity values of 1 and $0.0001 \mathrm{~m}^{2} / \mathrm{s}$ above and below a depth of $1 \mathrm{~km}$ (Figure 5.10c). Despite its simplicity, Model 5 accounts for both the observed movements towards the satellite on the southern side of the Greendale East Fault (C) and in the region between the western Charing Cross (D) and northern Greendale NW-SE (B) faults, and away from the satellite within the step-over and in the corner of the Greendale Central (A) and Greendale NW-SE Faults (B). Nevertheless, Model 5 cannot account for the observed signal entirely: in particular, movements away from the satellite measured on the Greendale NW-SE (B), Charing Cross (D), near Hororata, NNW from the epicentre and NE from step-over faults are not fully explained. 
Table 5.2: Model parameters of poroelastic rebound mechanism with different numbers of layers, depths of interface, values of diffusivity above and below the interface and Skempton's and Biot coefficients.

\begin{tabular}{|c|c|c|c|c|c|}
\hline Number of Layers & Top layer & Model & Diffusivity $\mathrm{m}^{2} / \mathrm{s}$ & Skempton's & Biot \\
\hline $\mathbf{9}$ & $\geq 1.75 \mathrm{~km}$ & $\begin{array}{c}\text { Model 1 } \\
\text { Model 6 }\end{array}$ & $\begin{array}{c}1 \\
0.1\end{array}$ & 0.5 & 0.6 \\
\hline $\mathbf{9}$ & $>1.75 \mathrm{~km}$ & $\begin{array}{c}\text { Model 2 } \\
\text { Model 7 }\end{array}$ & $\begin{array}{c}1 \\
0.1\end{array}$ & 0.5 & 0.6 \\
\hline $\mathbf{9}$ & $>1.0 \mathrm{~km}$ & $\begin{array}{c}\text { Model 3 } \\
\text { Model 8 }\end{array}$ & $\begin{array}{c}1 \\
0.1\end{array}$ & 0.5 & 0.6 \\
\hline $\mathbf{2}$ & $>1.75 \mathrm{~km}$ & $\begin{array}{c}\text { Model 4 } \\
\text { Model 9 }\end{array}$ & $\begin{array}{c}1 \\
0.1\end{array}$ & 0.5 & 0.6 \\
\hline $\mathbf{2}$ & $>1.0 \mathrm{~km}$ & $\begin{array}{c}\text { Model 5 } \\
\text { Model 10 }\end{array}$ & $\begin{array}{c}1 \\
0.1\end{array}$ & 0.5 & 0.6 \\
\hline $\mathbf{9}$ & $>1.75 \mathrm{~km}$ & $\begin{array}{c}\text { Model 11 } \\
\text { Model 12 }\end{array}$ & $\begin{array}{c}1 \\
0.1\end{array}$ & 0.6 & 0.8 \\
\hline
\end{tabular}

Figure 5.11 presents the displacement time series extracted from InSAR analysis (shown in Figure 5.4) at point "A" in Figure 5.1b and the corresponding poroelastic responses calculated with different model parametrisations. Although the effects of poroelastic rebound had reduced significantly by the time of the first InSAR acquisition, the signal persisted from 56 to 168 days after the earthquake. We infer that poroelastic rebound occurred during the early postseismic time interval, and that postseismic deformation continued even after poroelastic rebound terminated.

As previously explained, neither afterslip nor poroelastic rebound can account for the entirety of the observed postseismic displacement. To account for the residual deformation, we combine the poroelastic rebound mechanism (Model 5) with the process of dilatancy recovery at depths shallower than $4 \mathrm{~km}$. In this case, as described in Section 5.2.2, the effects of afterslip on the faults that had ruptured coseismically is presumed to accompany dilatancy recovery. The amount of afterslip required on each of the faults other than the Charing Cross Fault to fit the observations is negligible after taking into account poroelastic displacements. Maximum dilatancy recovery is estimated to have occurred on the Greendale NW-SE and Charing Cross Faults, with an approximate value of $7 \times 10^{4} \mathrm{~m}^{3}$. Minor recovery is modelled at the western end of the Greendale East Fault, the NE Fault from step-over and on the left-lateral NNW Fault from the epicentre (Figure 5.12). The dilatancy recovery can partially explain the signal observed along several faults and the step-over in the region. The signal along the Charing Cross Fault can also be induced by afterslip. The observed, modelled and residual displacements of the proposed model and also the amount of displacement due 

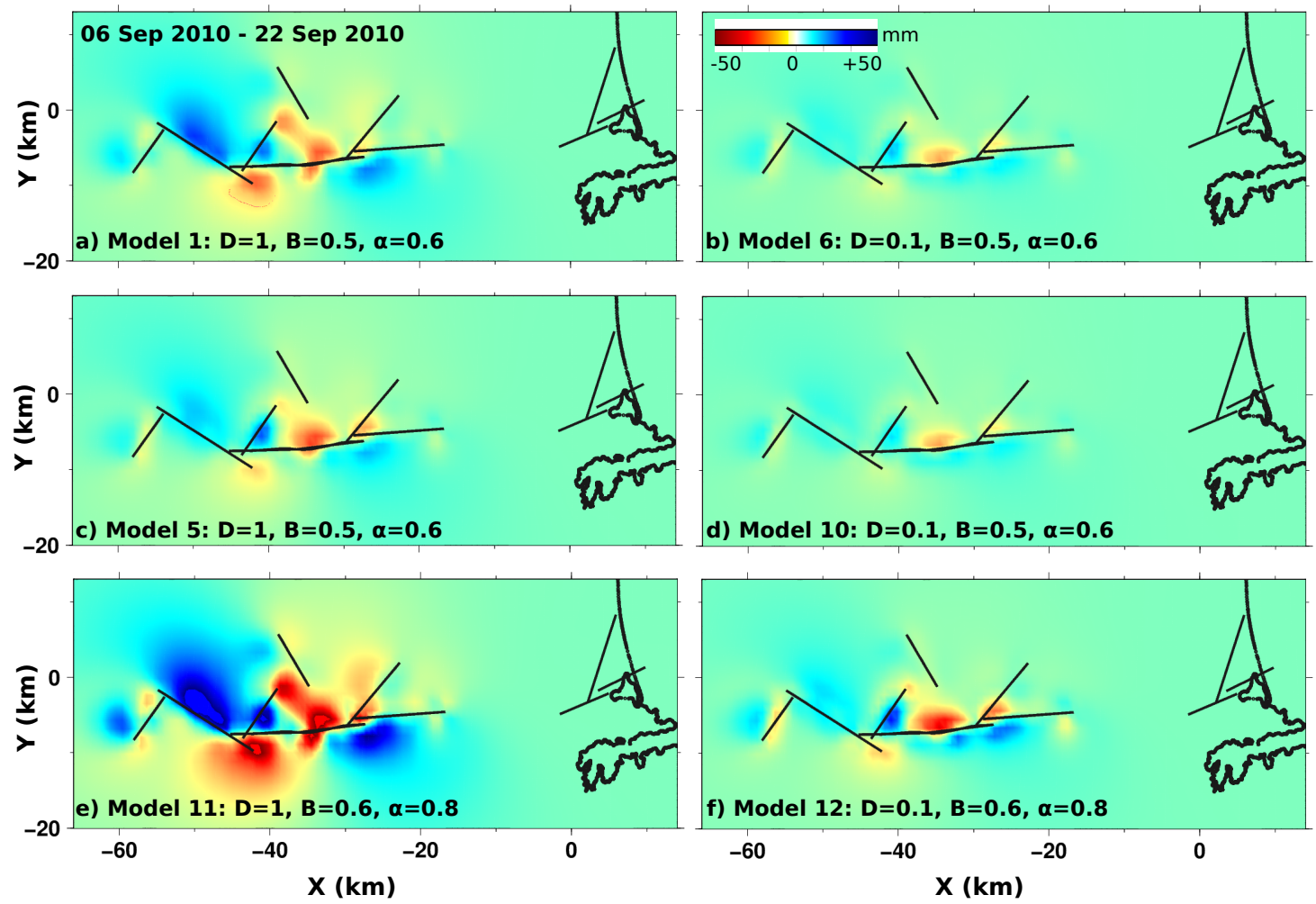

Figure 5.10: Estimated displacement induced by the poroelastic rebound mechanism following the Darfield earthquake in the LOS of ascending CSK with different values of poroelastic properties. a) Model 1: 9 layers with Skempton's 0.5, Biot 0.6 and diffusivity $1 \mathrm{~m}^{2} / \mathrm{s} . \mathrm{b}$ ) Model 6: 9 layers with Skempton's 0.5, Biot 0.6 and diffusivity $0.1 \mathrm{~m}^{2} / \mathrm{s}$. c) Model 5: Shallow 2 layers with Skempton's 0.5, Biot 0.6 and diffusivity $1 \mathrm{~m}^{2} / \mathrm{s}$. d) Model 10: Shallow 2 layers with Skempton's 0.5, Biot 0.6 and diffusivity $0.1 \mathrm{~m}^{2} / \mathrm{s}$. e) Model 11: 9 layers with Skempton's 0.6, Biot 0.8 and diffusivity $1 \mathrm{~m}^{2} / \mathrm{s}$. f) Model 12: 9 layers with Skempton's 0.6, Biot 0.8 and diffusivity $0.1 \mathrm{~m}^{2} / \mathrm{s}$. Red colours indicate movement away from satellite and blue colours indicate movement towards the satellite.

to the recovery process are shown in Figure 5.13. This combined model reduces the RMSE from $9 \mathrm{~mm}$, for poroelastic rebound only, to $5 \mathrm{~mm}$.

\subsection{Post-Christchurch displacement}

\subsubsection{GPS and InSAR observations}

In Figure 5.14, we present horizontal campaign GPS velocities acquired annually between early 2012 and 2015. We show mean LOS velocities computed from TSX data acquired between 26 January 2012 and 22 May 2014, an interval chosen to exclude coseismic effects on the calculated ground displacements of the 13 June 2011 and the 23 December 2011 earthquakes. 


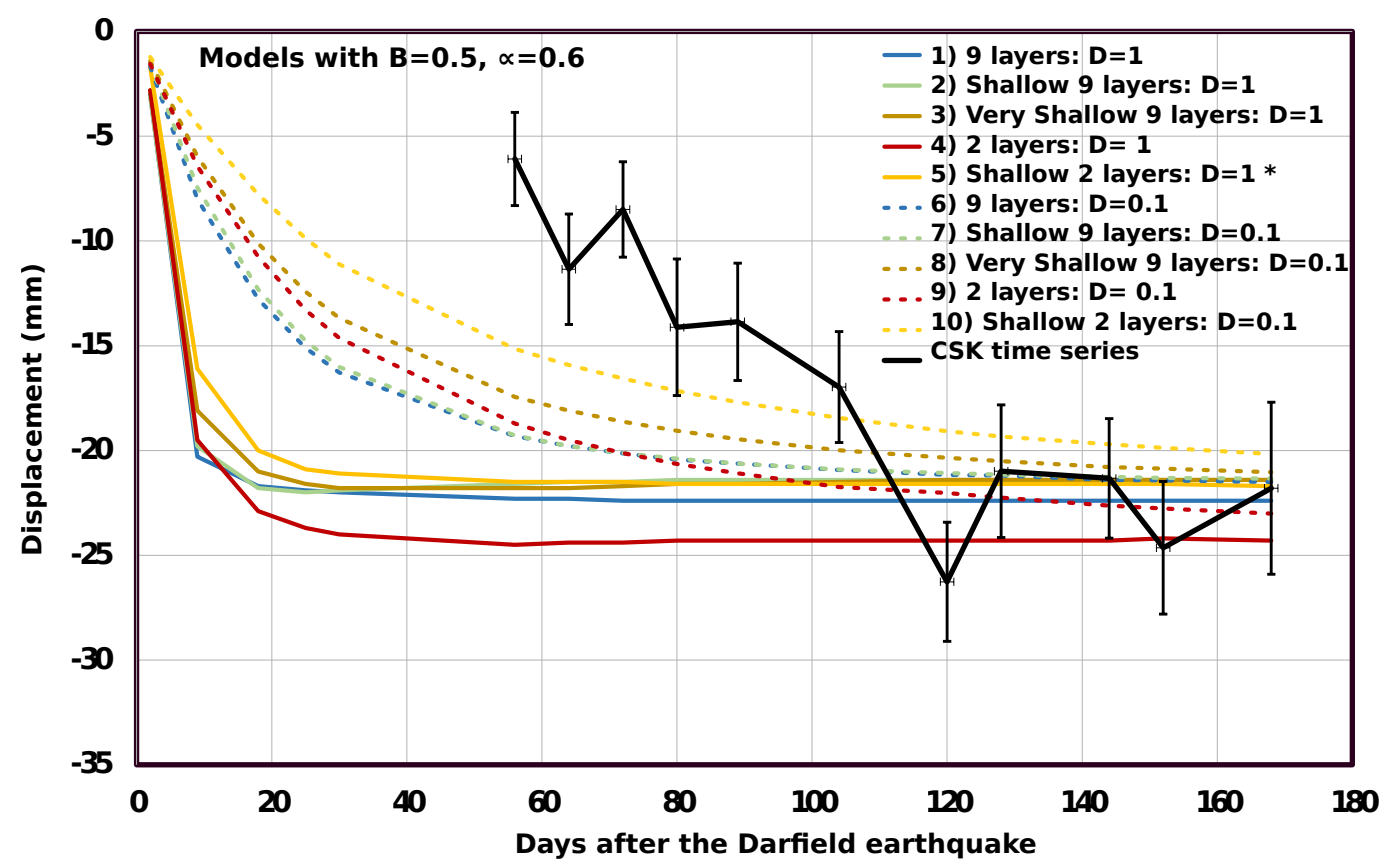

Figure 5.11: Observed (from ascending CSK time series) and estimated time series at point A" (Figure 5.1b) within the Greendale Fault step-over induced by different multi-layered poroelastic rebound with two diffusivity values of 1 and $0.1 \mathrm{~m}^{2} / \mathrm{s}$, and the values of 0.5 and 0.6 for Skempton's and Biot coefficients. Model 5 is the preferred spatial model (see Figure 5.10).

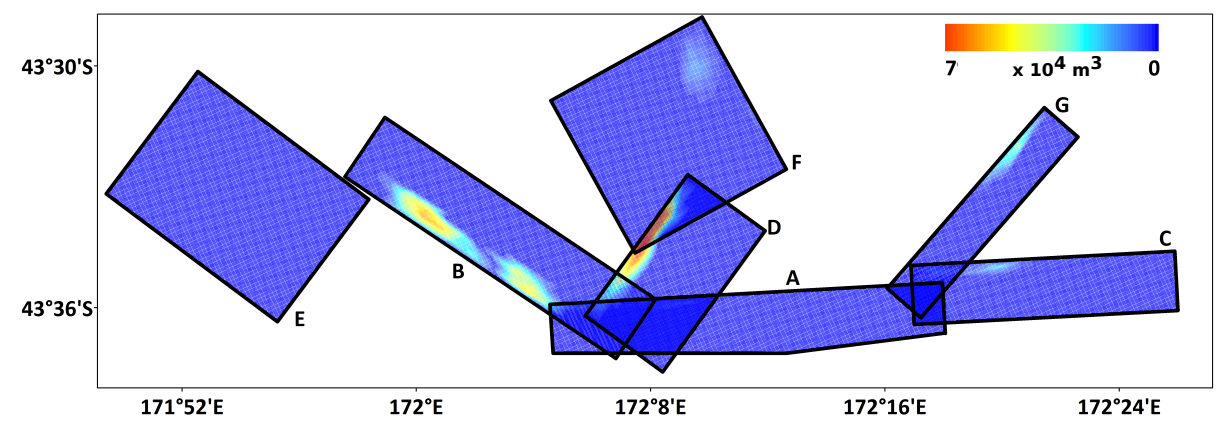

Figure 5.12: Regular grid of Mogi point sources on the ruptured fault planes during the Darfield earthquake. Red colour indicates the volume decrease of each Mogi point source. Other faults are described in Figure 5.1b.

Three key features are evident in the velocity results extracted from GPS velocities and InSAR time series:

- Movement away from the satellite at a velocity of $\sim 3 \mathrm{~mm} / \mathrm{yr}$ in the Christchurch urban region and along the coast;

- A ground displacement anomaly across the WSW continuation of the Western Christchurch Fault $(\mathrm{H})$, which coincides with the lineation of aftershocks recognised following the Darfield and Christchurch earthquakes (Sibson et al., 2011; Syracuse et al., 2012; Herman et al., 2014). Beavan et al. (2012a) discussed the possibility of seismic or aseismic slip inducing westward and eastward motions at 
t!]

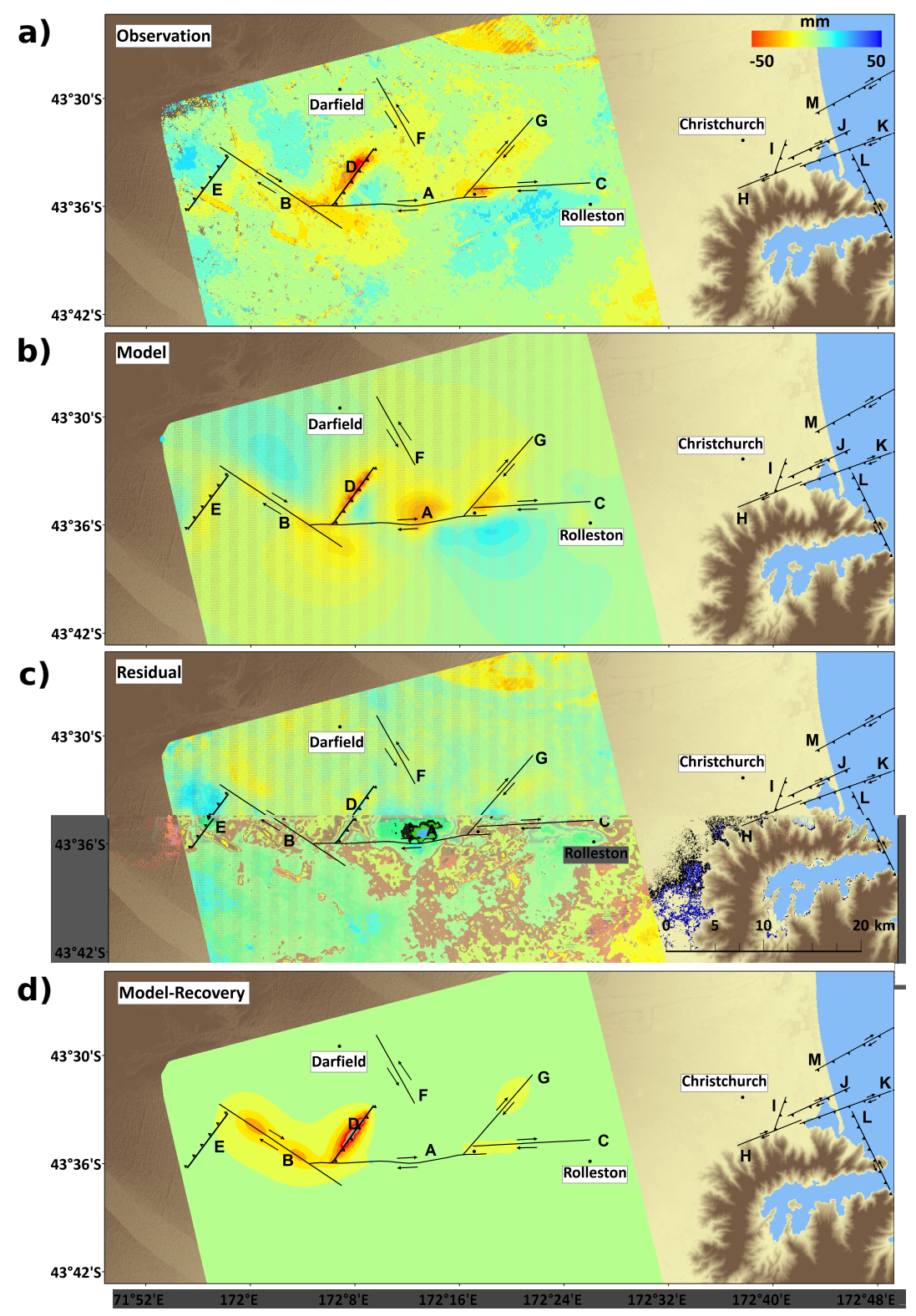

Figure 5.13: a) Observed ground displacement measured by ascending CSK as in Figure 5.3a. b) Estimated displacement induced by the combined dilatancy recovery and poroelastic rebound projected in the LOS of ascending CSK. c) Residual displacement induced by the combined dilatancy recovery and poroelastic rebound projected in the LOS of ascending CSK. d) Estimated displacement induced by the process of dilatancy recovery only. 


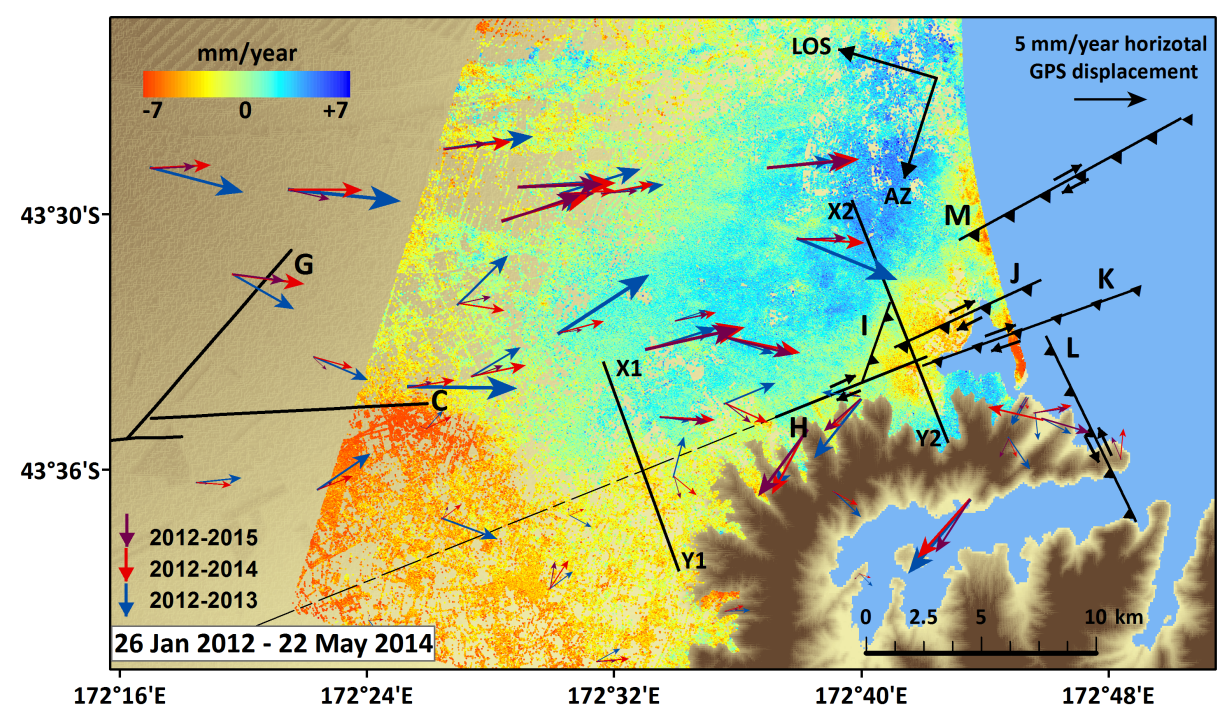

Figure 5.14: Observed mean LOS velocity acquired from TSX acquisitions following the Christchurch earthquake. Arrows represent GPS horizontal velocities in the Pacific plate reference frame relative to the continious GPS site MQZG for three periods: early 20122013 (blue), 2012-2014 (red) and 2012-2015 (maroon). Red colours indicate movement away from the satellite and blue colours indicate movement towards the satellite. Dashed line is microearthquake lineament (inferred to be the continuation of fault Segment H). Other faults are described in Figure 5.1b.

continuous GPS sites MQZG and SWAN, respectively (location of cGPS sites in Figure 5.1b). The relative motion between MQZG and SWAN is in agreement with the right-lateral velocity observed with InSAR and GPS.

- Movement away from the satellite at the easternmost end of the Greendale East Fault.

The second and third features are likely to be associated with the long-term postDarfield displacement. Too few acquisitions spanning the eastern half of the Greendale East Fault were made following the Darfield and prior to the Christchurch earthquakes to analyse the post-Darfield displacement at different timescales.

\subsubsection{Modelling results}

\section{First-order approximation}

The viscoelastic responses to the Darfield and Christchurch earthquakes are calculated and projected in the LOS of the TSX satellite for the period between 26 January 2012 and 22 May 2014.

We cannot account for the observed velocity, measured by TSX acquisitions, in terms of the insignificant velocity associated with plausible viscoelastic responses $(<2 \mathrm{~mm} / \mathrm{yr})$. 
In order to interpret the observed displacement features, we instead consider ratestrengthening afterslip (Barbot et al., 2009) on the lineament and the coseismic faults that had ruptured during the Christchurch earthquake. The lineament is assumed to be a continuation of the Western Christchurch Fault ( $\mathrm{H}$ in Figure 5.1b) and to have a length of $\sim 15 \mathrm{~km}$, a dip of $070^{\circ} \mathrm{SSE}$ and a strike of $067^{\circ}$. The extent of the microseismicity defines the length of the lineament (extension of $\mathrm{H}$ ).

Assuming values of $20 \mathrm{~mm} / \mathrm{yr}$ and $5 \mathrm{MPa}$ for $\dot{s_{0}}$ and $a \sigma$, respectively, we first solve for the shear stress changes and afterslip on the lineament at successive times. The values are optimised to obtain a similar magnitude of discontinuity across the lineament.

Figures 5.15a and b represent the strike and dip components of afterslip on the lineament at four epochs between 137 days and 1095 days after the Christchurch event. Afterslip on the lineament is mostly concentrated on its easternmost $\sim 4 \mathrm{~km}$ edge, adjacent to the coseismic patch. The computed afterslip model involves predominantly right-lateral motion with a maximum value of $\sim 50 \mathrm{~mm}$ at the final epoch (three years after the earthquake). This is consistent with the faulting geometry of the Greendale Fault and other recently recognised eastwest-striking faults in the Canterbury region (Gledhill et al., 2011; Kaiser et al., 2012; Quigley et al., 2012; Beavan et al., 2010b, 2011; Bannister et al., 2011; Syracuse et al., 2012; Sibson et al., 2011; Bannister and Gledhill, 2012; Beavan et al., 2012a). Modelled surface displacement along the lineament reaches a maximum of $\sim 10 \mathrm{~mm}$ within 1095 days of the Christchurch earthquake (Figure 5.15).

We apply the same method using the previous constitutive parameters to estimate the rate-strengthening afterslip on the combination of the nearby faults and the lineament. Figure 5.16 illustrates the cumulative ground displacement at the final epoch three years after the Christchurch earthquake. The net ground displacement modelled by afterslip exhibits eastward displacement on the northern side of the lineament and the opposite direction of motion in the southern side (Figure 5.16a2). The horizontal motion is explained by continued right-lateral strike-slip faulting. Northward motion is computed by the model on the northern side of the Eastern Christchurch Fault (Figure 5.16a3). An uplift signal is estimated on the eastern side and a subsidence signal on the western side of the NNE Fault (I) due to the reverse afterslip (Figure 5.16a4). The afterslip model appears to reproduce the sense and magnitude of displacement associated with the WSW extension of fault segment $(\mathrm{H})$ delineated by aftershock activity (Figure 5.16c). However, the afterslip model cannot explain the subsidence signal observed in the urban area of Christchurch or along the coast. The computed LOS displacement after three years induced by afterslip on fault segments (I) and (J) show 
net uplift in that area (Figure 5.16d). The profiles of estimated velocity in the corresponding interval due to the rate-strengthening afterslip (red) on the lineament and nearby faults along X1Y1 and X2Y2 (Figure 5.14) are shown with the corresponding observations (grey) in Figure 5.16c and d, respectively.

Based on the results of the afterslip modelling, we conclude the following:

- Right-lateral frictional afterslip on the extension of fault segment $(\mathrm{H})$ is localised near the westernmost end of the Western Christchurch Fault. The observed discontinuity across the lineament seems to extend further towards the easternmost end of the Greendale East Fault (C) demonstrated by velocity of the descending TSX time series and GPS velocities (Figure 5.14); and

- Frictional afterslip on the faults that ruptured coseismically during the Christchurch earthquake does not account for the observed subsidence in the Christchurch urban region or along the coast.

\section{Inversion of GPS and InSAR velocities}

As previously discussed, a broad eastward displacement is observed in the GPS data, in three successive years, and in descending TSX InSAR timeseries. A forward model of afterslip on the Christchurch faults and the lineament accounts for neither the subsidence in the urban region of Christchurch, nor the uniform discontinuity across the lineament. Therefore, in order to understand the observed discontinuity, we invert the ground displacement measurements in terms of afterslip on the Christchurch faults (Figure 5.14 H, J and M), the lineament (extension of H), the eastern end of the Greendale East (Figure 5.14 C) Fault, and NE Fault from step-over (Figure $5.14 \mathrm{G}$ ). In the afterslip models, ground velocities are calculated relative to the continuous GPS site MQZG (location shown in Figure 5.1).

The broad eastward movement continues on the northern side of the faults towards the eastern end of the fault segments associated with the Darfield earthquake. This signal may imply that afterslip is activated not only by the Christchurch earthquake, but also by the preceding Darfield earthquake. The unavailability of long-term observations following the Darfield earthquake, spanning the entire region including the fault segments associated with both earthquakes, means that this hypothesis cannot be verified.

We consider four afterslip models: 1) single fault model on the lineament; 2) six fault model on the lineament as well as NE Fault from step-over $(\mathrm{G})$, Greendale East Fault (C), SW-NE Fault (M), and Western and Eastern Christchurch Faults (H and J, respectively); 3) three fault model on the lineament, NE Fault from step-over and Greendale 

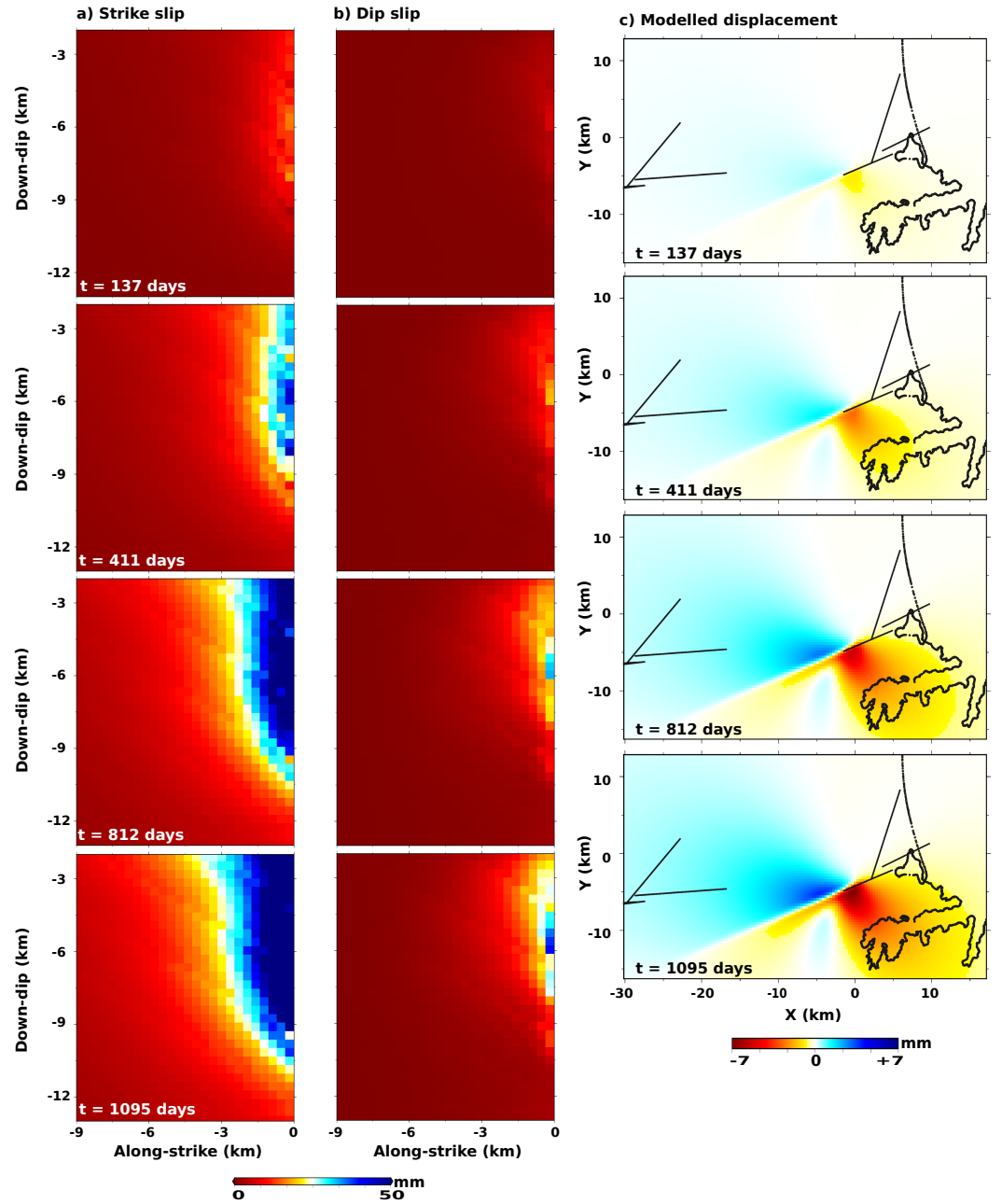

Figure 5.15: Estimated a) Strike-slip and b) Dip-slip magnitudes on the subsurface lineament at successive times following the Christchurch earthquake assuming rate-strengthening rheology on the fault with the values of $20 \mathrm{~mm} / \mathrm{yr}$ and $5 \mathrm{MPa}$ for $\dot{s_{0}}$ and $a \sigma$, respectively for constitutive parameters. c) Surface displacement projected in the LOS at successive times following the Christchurch earthquake based on a model of rate-strengthening afterslip on the subsurface lineament. Red colours indicate movement away from satellite and blue colours indicate movement towards the satellite.

East Fault; and 4) five fault model on the NE Fault from step-over (G), Greendale East Fault (C), SW-NE Fault (M) and Western and Eastern Christchurch Faults. The magnitudes and rakes of afterslip for each model (rows) and each epoch (columns) are illustrated in Figure 5.17. The second epoch between early 2012 and 2014 is constrained by the InSAR data between January 2012 and May 2014. The velocity of observations (blue) and models (red) are shown in Figure 5.18, 5.19 and 5.20 for each model and three epochs, respectively.

Major right-lateral slip is inferred to reproduce the eastward movement on the northern side of the faults. The amounts of reverse-slip on the SW-NE (M) and Eastern Christchurch Faults are consistent with the oblique coseismic slip. We compare the RMSE of the slip models in Table 5.3. The single fault afterslip model does not re- 

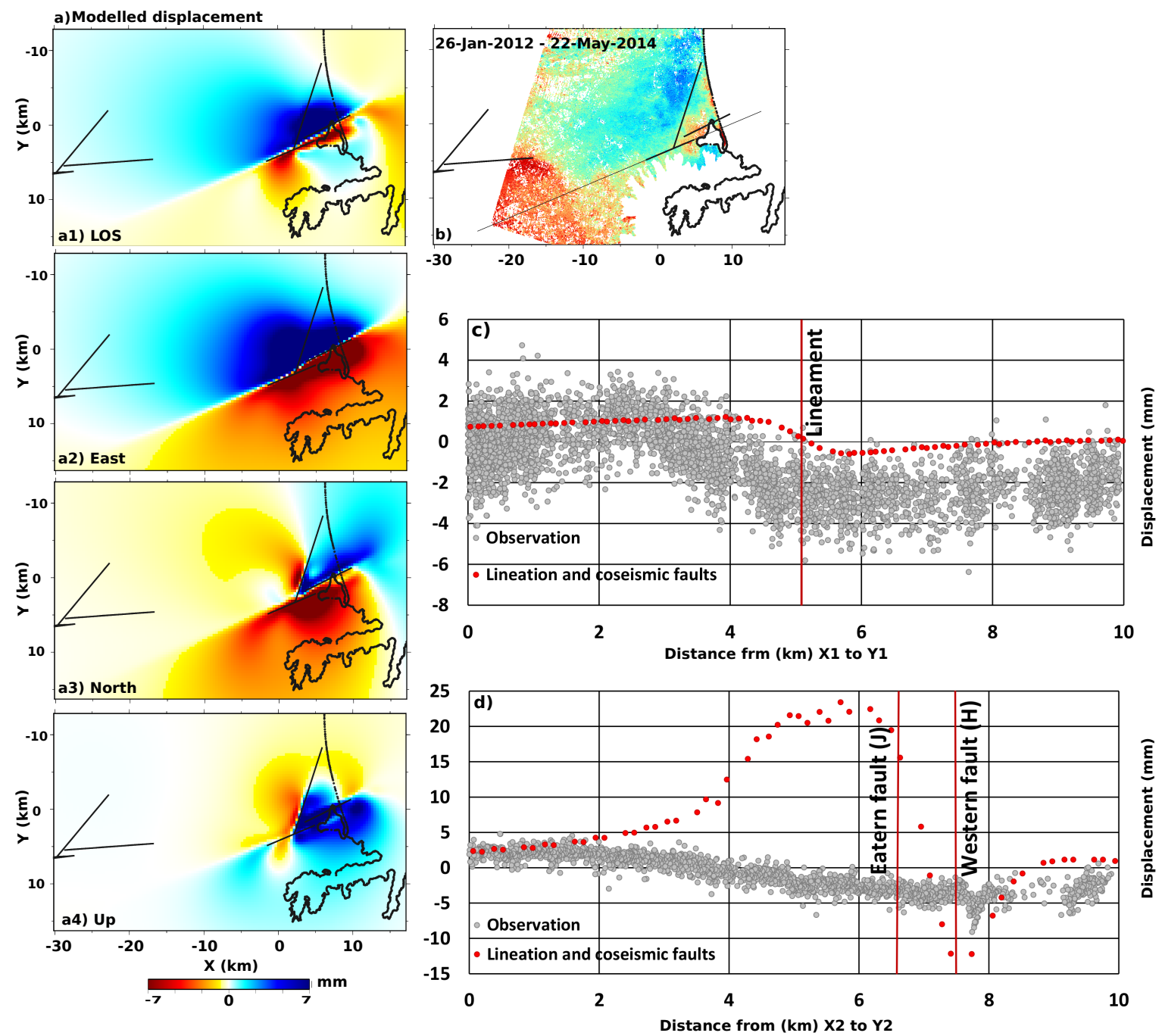

Figure 5.16: a) Surface displacement produced by accumulated afterslip on the previously ruptured faults during the Christchurch earthquake and on the subsurface lineament in the three years following the Christchurch earthquake, a1) LOS, a2) East, a3) North and a4) Vertical components of the estimated ground displacement. b) Observed LOS velocity from TSX following the Christchurch earthquake as in Figure 5.14. Red colours indicate movement away from satellite and blue colours indicate movement towards the satellite. c) Profile of estimated velocity, in the corresponding interval, and observation (grey) along the X1Y1 line presented in Figure 5.14. d) Profile of estimated velocity and observation along the X2Y2 line presented in Figure 5.14.

produce the observations sufficiently. Model 2 predicts the observations with slightly lower RMSE values in different epochs compared to other models. 

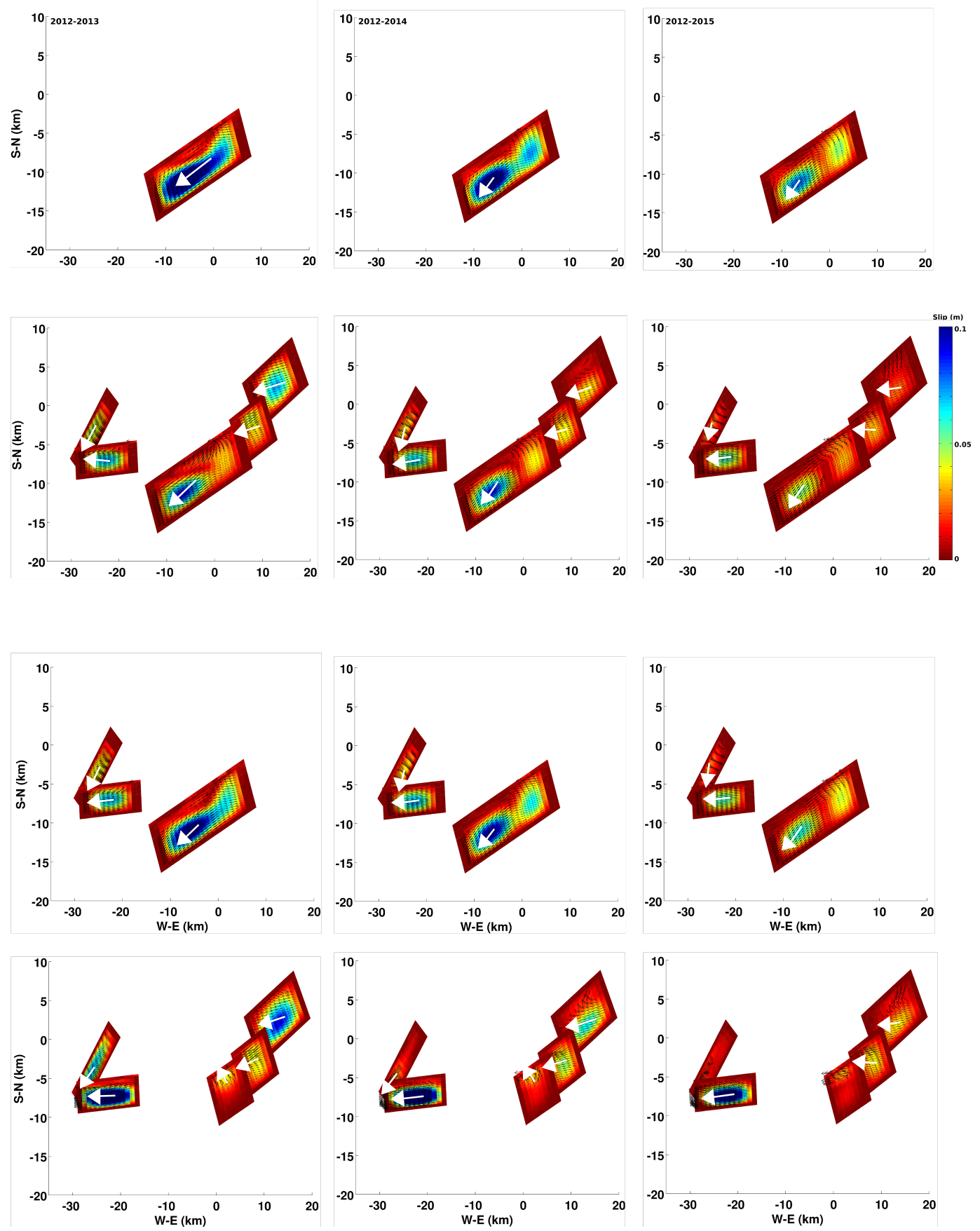

Figure 5.17: Magnitudes and rake directions of four afterslip models (rows) for each epoch (columns). Models: 1) on the lineament; 2) on the lineament as well as NE Fault from stepover (G), Greendale East Fault (C), SW-NE Fault (M) and Western and Eastern Christchurch Faults (H and J, respectively); 3) on the lineament, NE Fault from step-over and Greendale East Fault; 4) on the NE Fault from step-over, Greendale East Fault, SW-NE Fault and Western and Eastern Christchurch Faults. The second epoch between early 2012 and 2014 is constrained by the InSAR timeseries velocity between January 2012 and May 2014. 

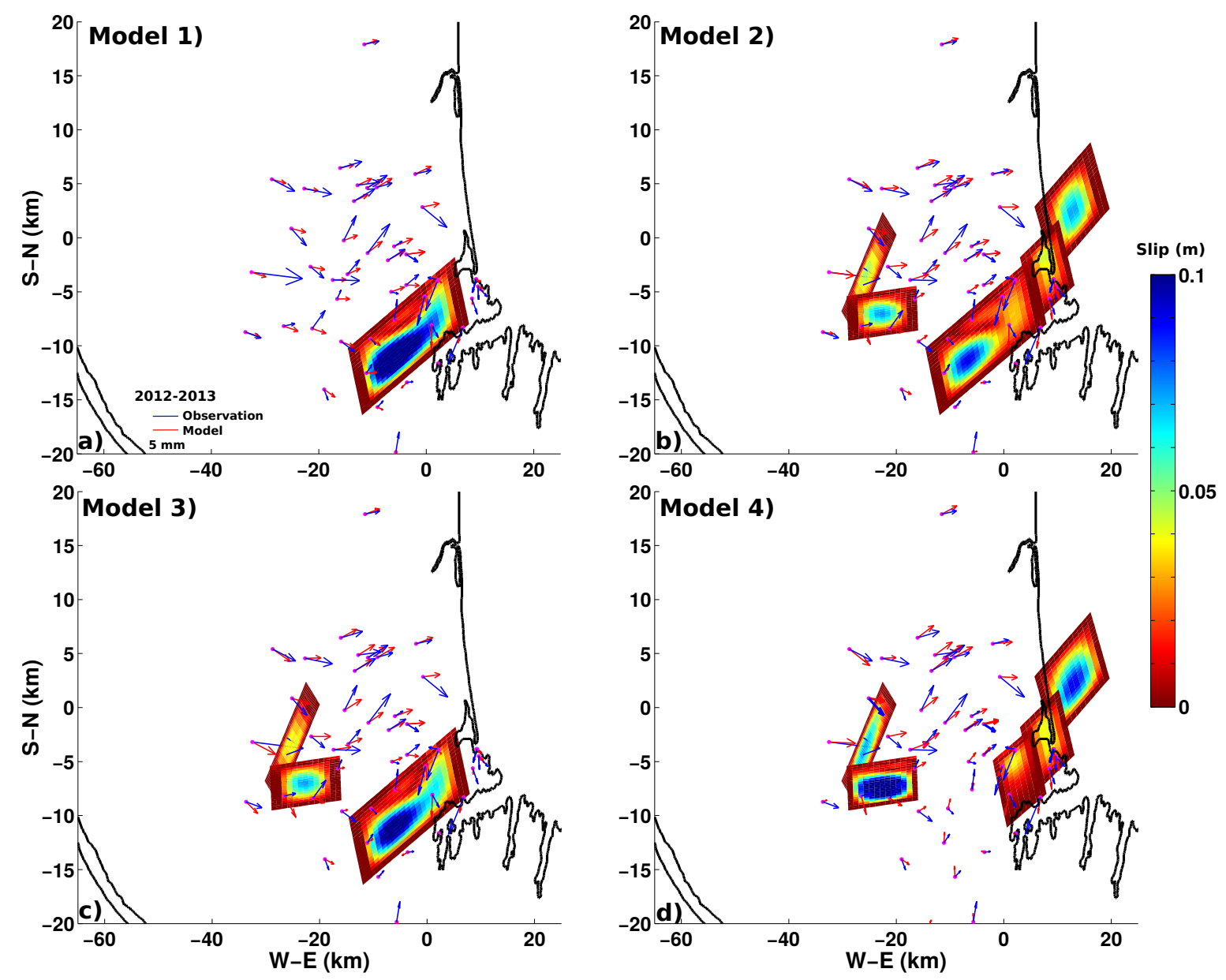

Figure 5.18: Velocity of observations and models are shown in blue and red, respectively for each model (epoch 2012-2013). Models: 1) on the lineament; 2) on the lineament as well as NE Fault from step-over (G), Greendale East Fault (C), SW-NE Fault (M) and Western and Eastern Christchurch Faults (H and J, respectively); 3) on the lineament, NE Fault from step-over and Greendale East Fault; 4) on the NE Fault from step-over, Greendale East Fault, SW-NE Fault and Western and Eastern Christchurch Faults. 

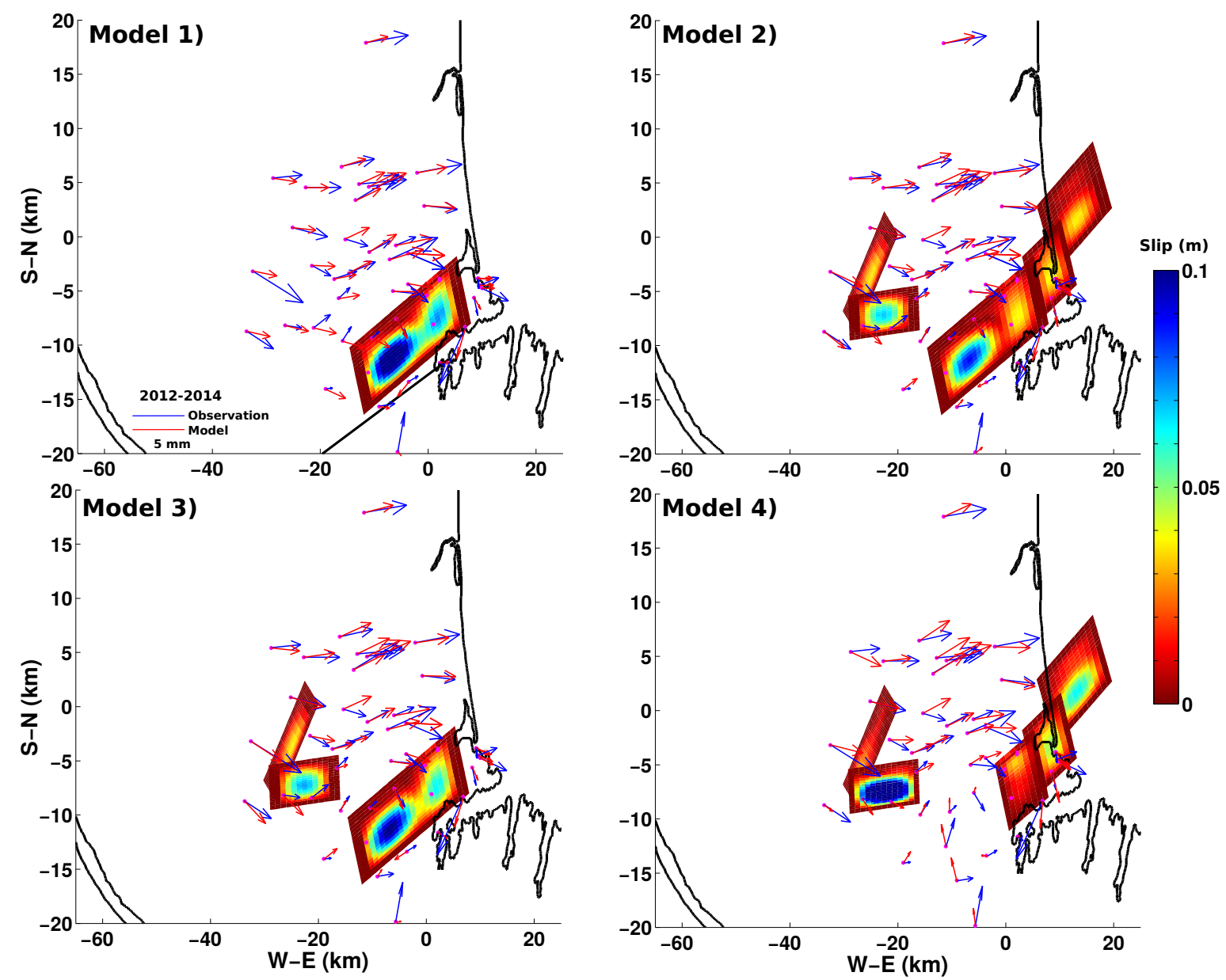

Figure 5.19: Velocity of observations and models are shown in blue and red, respectively for each model (epoch 2012-2014). Models: 1) on the lineament; 2) on the lineament as well as NE Fault from step-over (G), Greendale East Fault (C), SW-NE Fault (M) and Western and Eastern Christchurch Faults (H and J, respectively); 3) on the lineament, NE Fault from step-over and Greendale East Fault; 4) on the NE Fault from step-over, Greendale East Fault, SW-NE Fault and Western and Eastern Christchurch Faults. Models are constrained by the InSAR timeseries velocity between January 2012 and May 2014.

\section{Subsidence signal in the urban region of Christchurch}

We consider the observed subsidence signal in the urban region of Christchurch and along the coast to be the result of either 1) poroelastic rebound; and/or 2) dilatancy recovery as observed following the Darfield earthquake; and/or 3) other sources such as liquefaction (e.g. Cubrinovski et al., 2011; Cubrinovski and Taylor, 2011; Mucciarelli, 2011; Cubrinovski et al., 2012; Quigley et al., 2013; Van Ballegooy et al., 2014). One method of studying areas affected by soil liquefaction involves the use of InSAR coherence maps, as Atzori et al. (2012) did after the February and June Christchurch earthquakes of 2011. The subsidence signal detected in Christchurch is located within the liquefaction affected areas. Displacement velocities were measured within a time interval of more than two years after the last event in December 2011. Therefore, the impact of the soil liquefaction had likely diminished by this time and poroelastic 

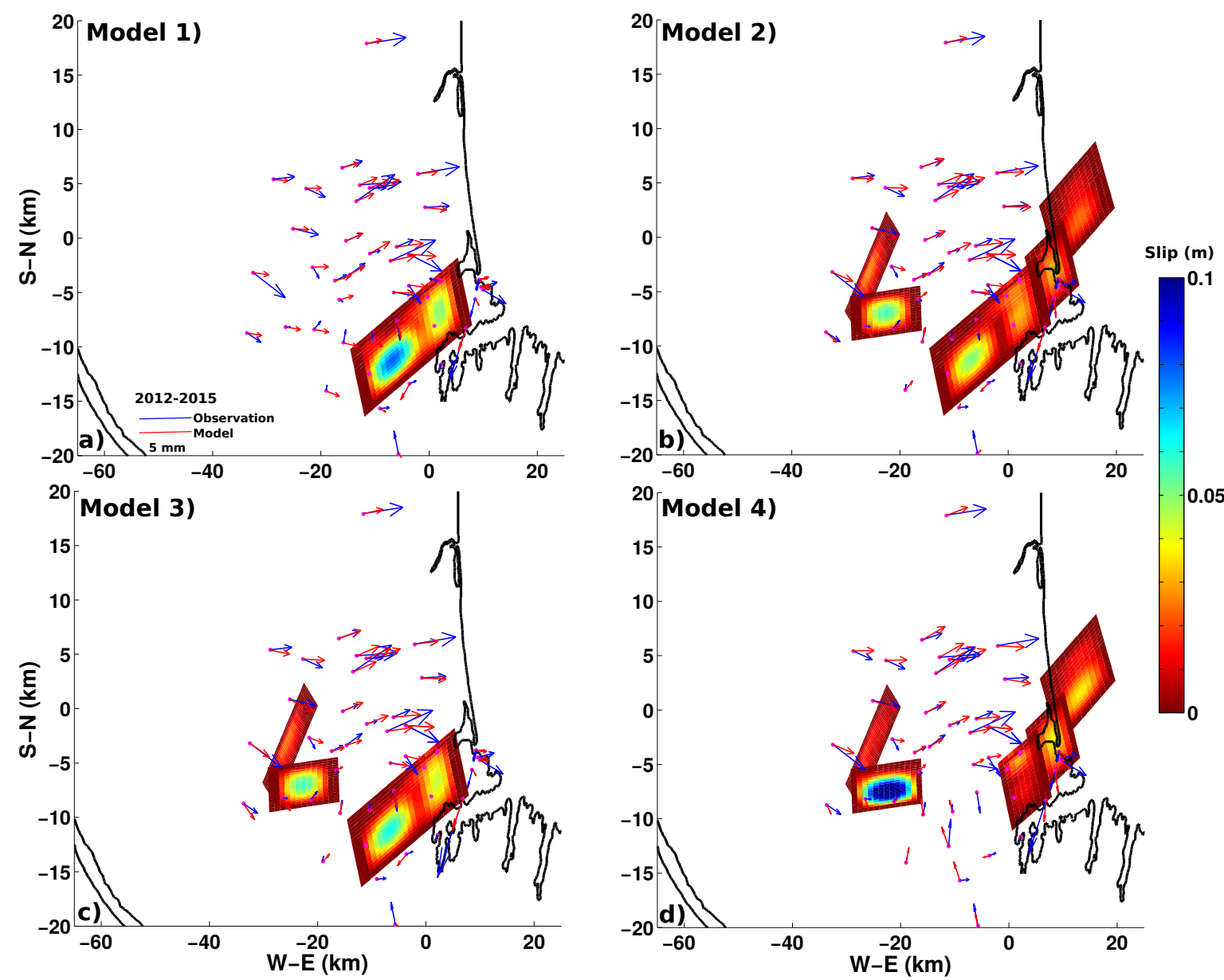

Figure 5.20: Velocity of observations and models are shown in blue and red, respectively for each model (epoch 2012-2015). Models: 1) on the lineament; 2) on the lineament as well as NE Fault from step-over (G), Greendale East Fault (C), SW-NE Fault (M) and Western and Eastern Christchurch Faults (H and J, respectively); 3) on the lineament, NE Fault from step-over and Greendale East; 4) on the NE Fault from step-over, Greendale East, SW-NE Fault and Western and Eastern Christchurch Faults.

rebound or dilatancy recovery are likely the principal causes of urban subsidence.

Two values for hydraulic diffusivity of $0.1 \mathrm{~m}^{2} / \mathrm{s}$ above the depth of $1.0 \mathrm{~km}$ and a small value of $0.0001 \mathrm{~m}^{2} / \mathrm{s}$ below the depth of $1.0 \mathrm{~km}$ are considered, assuming any poroelastic rebound to be a shallow process. We assume values of 0.6 and 0.89 for Skempton's and Biot coefficients in order to allow the poroelastic rebound effects to last until the final acquisition of the InSAR timeseries in May 2014. Figure 5.21 illustrates the ground displacement produced by poroelastic rebound at four epochs. A zone of subsidence is computed, but the corresponding velocity in the observed time interval is insignificant in the area bounded by the Eastern $(\mathrm{J})$ and NNE (I) fault planes $(<1 \mathrm{~mm} / \mathrm{yr})$.

We next examine the effects of possible dilatancy recovery in Christchurch, in order to interpret the subsidence signal using the ground velocity measured by InSAR time series. A regular grid of Mogi point sources is modelled at depths shallower than $4 \mathrm{~km}$. 
Table 5.3: Root Mean Square Residuals in three epochs of four slip models: 1) on the lineament; 2) on the lineament as well as NE Fault from step-over, Greendale East Fault, SW-NE Fault and Western and Eastern Christchurch Faults; 3) on the lineament, NE Fault from step-over and Greendale East Fault; and 4) on the NE Fault from step-over, Greendale East Fault, SW-NE Fault and Western and Eastern Christchurch Faults.

\begin{tabular}{l|c|c|c} 
& $\mathbf{2 0 1 2 - 2 0 1 3}$ & $\mathbf{2 0 1 2 - 2 0 1 4}$ & $\mathbf{2 0 1 2 - 2 0 1 5}$ \\
\hline Model 1 (mm) & 2.7 & 1.9 & 1.9 \\
Model 2 (mm) & 2.3 & 1.6 & 1.6 \\
Model 3 (mm) & 2.5 & 1.7 & 1.7 \\
Model 4 (mm) & 2.4 & 1.8 & 1.7
\end{tabular}

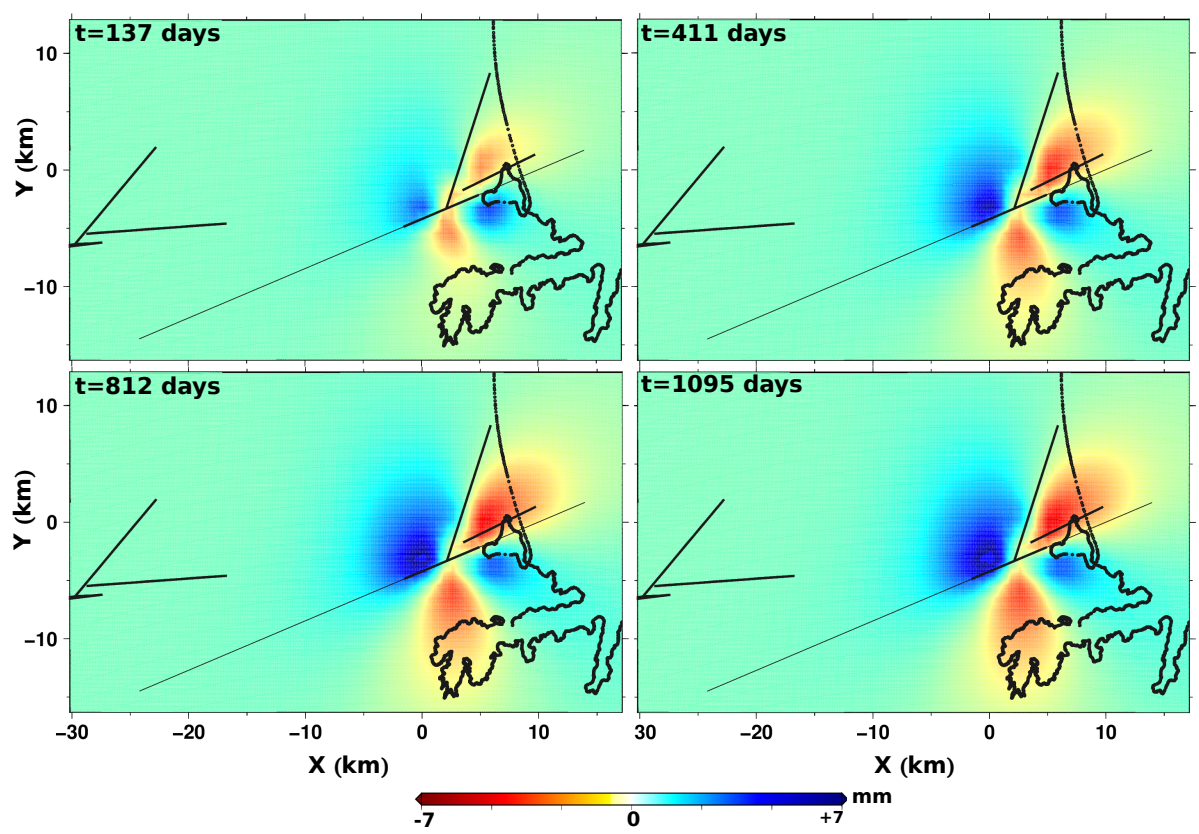

Figure 5.21: Modelled ground displacement associated with poroelastic rebound with a diffusivity value of $0.1 \mathrm{~m}^{2} / \mathrm{s}$ and the values of 0.6 and 0.89 for Skempton's and Biot coefficients, projected in the LOS direction. Red colours indicate movement away from satellite and blue colours indicate movement towards the satellite.

The Mogi point sources are located on the fault planes related to the February, June and December 2011 earthquakes (H, I, J, K, L and M). A maximum volume decrease of $\sim 1.8 \times 10^{4} \mathrm{~m}^{3}$ is inferred on the December SW-NE Fault. Figure 5.22 summarises the results.

We combine the ground velocity predicted by Model 2 (best RMSE: Table 5.3) between early 2012 and 2014 as the result of inversion on both GPS and InSAR velocities and the estimated velocity resulting from dilatancy recovery. We present the observed, estimated, and residual displacements of combined afterslip with dilatancy recovery in Figure 5.23.a, b and c, respectively. 


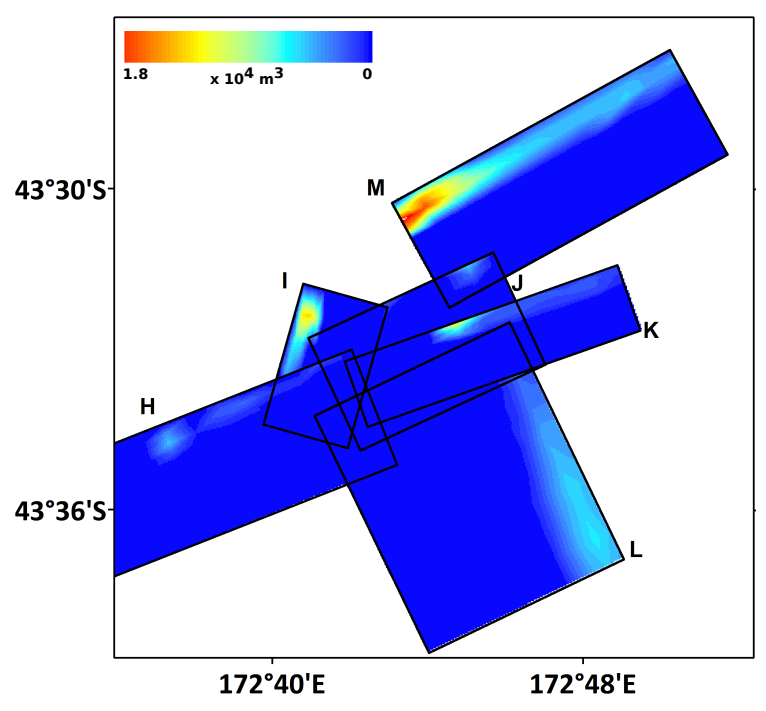

Figure 5.22: Volume decreases of a grid of regularly spaced contracting Mogi point sources on the fault planes that ruptured by the 22 February, 13 June and 23 December 2011 Christchurch earthquakes.

\subsection{Discussion}

Significant orbital and atmospheric artefacts in descending acquisitions of the CSK and TSX satellites make it difficult to determine the sense of postseismic ground motion immediately following the Darfield earthquake (Figure 5.3b and 5.5). However, we can infer from the profiles in Figure $5.7 \mathrm{a}$ and $\mathrm{b}$ that the movement towards the satellite, observed by ascending and descending interferograms, in the southern side of the Greendale East Fault is mainly a vertical motion.

We have considered possible rate-strengthening afterslip on the faults that ruptured during the mainshock. The results (Figure 5.8) imply that the afterslip model cannot thoroughly account for the spatial variability of short-term observations.

Pore pressure changes induced coseismically induce fluid flow. The increase of the groundwater levels observed in unconfined and semi-confined aquifers in the Canterbury region within a day of the Darfield earthquake provide evidence of poroelastic rebound (Cox et al., 2012). We compare the temporal ground displacement estimated by poroelastic rebound with values of $0.5,0.6$ and $1 \mathrm{~m}^{2} / \mathrm{s}$ for Skempton's and Biot coefficients and hydraulic diffusivity, respectively, with the time series of measured displacement over the fault step-over in Figure 5.11 and the unwrapped interferogram in Figure 5.3. These comparisons show that the mechanism of poroelastic rebound does not thoroughly estimate the time-dependent surface displacement within the five months between the Darfield and Christchurch earthquakes. 

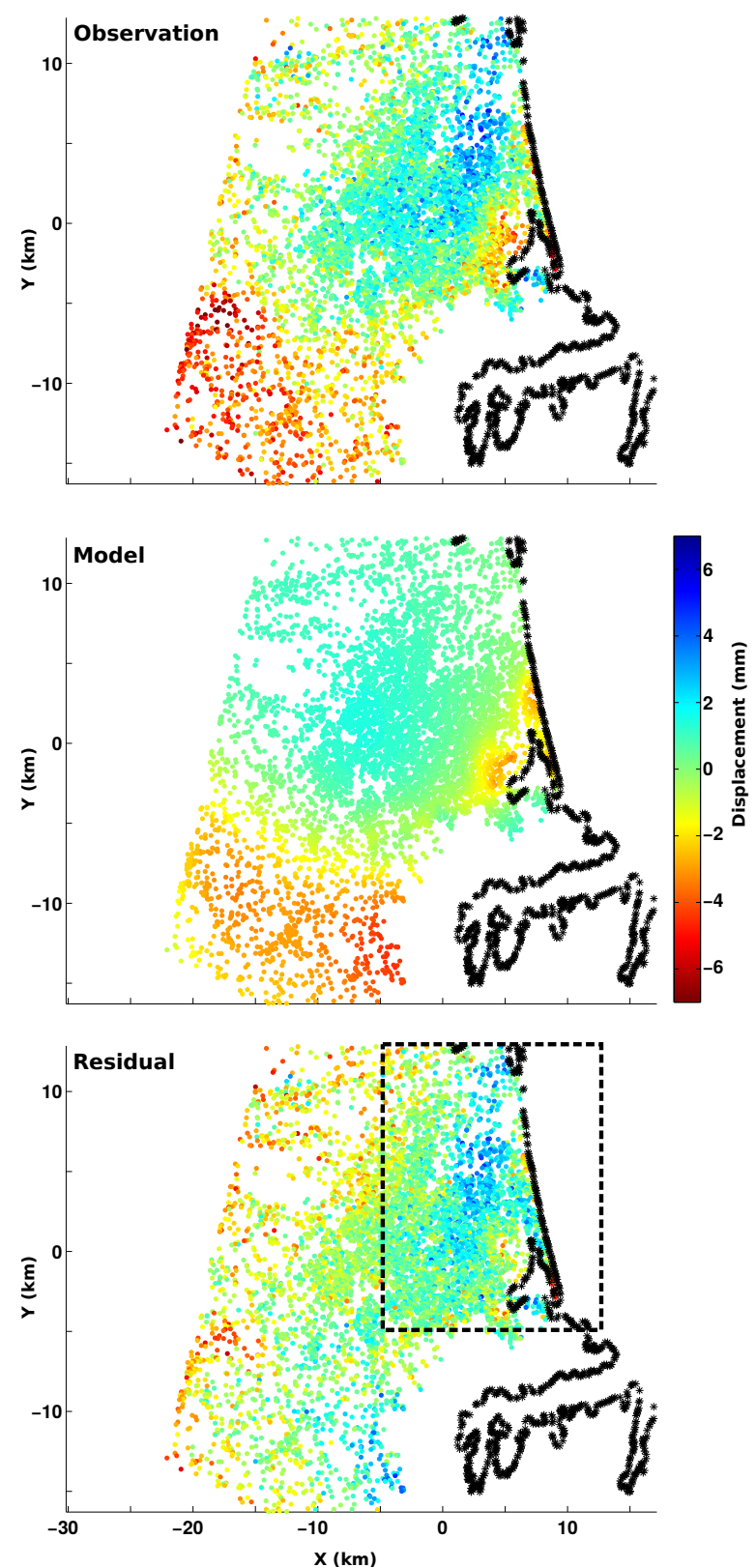

Figure 5.23: a) Observed ground velocity measured by descending TSX as in Figure 5.6b. b) Modelled LOS velocity for TSX produced by combined afterslip (Model 2) and dilatancy recovery. c) Residuals of the model produced by combined afterslip (Model 2) and dilatancy recovery following the Christchurch earthquake. Red colours indicate movement away from satellite and blue colours indicate movement towards the satellite.

We interpret the main observational features after the Darfield earthquake to be induced by dilatancy recovery due to weakening of the crust during the mainshock (Reyners et al., 2014) and poroelastic rebound.

Following the Christchurch earthquake, afterslip yields a broad discontinuity, across the lineament, observed by GPS and InSAR velocities. The ongoing movement between early 2012 and 2015 implies that afterslip persisted after the Christchurch earthquake and associated large aftershocks. Two questions remain unanswered by our analysis: 
1) the western extent of afterslip and the corresponding displacement towards the fault segments associated with the Darfield earthquake; and 2) the time of the onset of afterslip following the Canterbury earthquake sequence of 2010-2012.

We have considered afterslip on five fault segments associated with the Darfield and Christchurch earthquakes as well as the proposed subsurface lineament, to explain the observed discontinuity. Model 2 in Table 5.3 provides the best fit with observations. This model involves slip on the lineament in addition to five fault segments.

The strike of the observed aftershock lineament of $067^{\circ}$ forms a non-Andersonian angle of $\sim 48^{\circ}$ with the orientation of the axis of maximum horizontal compressive stress (Townend et al., 2012; Holt et al., 2013). This geometry may either mean that there is a possible dip component of slip on the proposed lineament, so the subsurface lineament is not purely right-lateral, or that the lineament is frictionally weak. Sibson et al. (2011) recognised this lineament by the diffuse aftershock concentration south of the Darfield earthquake surface rupture (see Bannister et al., 2011; Gledhill et al., 2011; Bannister and Gledhill, 2012).

The subsidence signal observed within the urban region of Christchurch is located in the area of shallow confined aquifers along the coast (Cox et al., 2012). The hydrological responses in aquifers and/or liquefaction are likely to have affected the velocity of postseismic ground movement. In addition to hydrological effects of the Christchurch earthquake and aftershocks, dilatancy recovery and/or poroelastic rebound may account for the observed movement away from the descending TSX in the region.

\subsection{Conclusions}

We have examined several alternative mechanisms of postseismic displacement in order to explain geodetic observations made following the Darfield and Christchurch earthquakes. We infer that processes of poroelastic rebound and dilatancy recovery occurred immediately following the Darfield earthquake. Afterslip may also have occurred on the Charing Cross Fault (D), but afterslip does not account for spatial variability of observations. The subsequent Christchurch earthquake was followed by two large aftershocks on 13 June and 23 December 2011 as well as ongoing afterslip (on the lineament and fault segments G, C, M, H and I) observed in three successive years between early 2012 and 2015. Afterslip is inferred to have occurred on the lineament forming an extension of the Western Christchurch Fault and defined by a band of seismicity. The SW-NE December, Eastern and Western Christchurch Faults along with Greendale East and 
NE Fault from step-over are likely to have also undergone postseismic afterslip. Finally, we conclude the observed subsidence signal in Christchurch to be the result of hydrological effects following the earthquake, such as liquefaction or mechanisms of poroelastic rebound and dilatancy recovery. 


\section{Chapter 6}

\section{Summary and recommendations}

\subsection{Post-Dusky Sound displacement}

In Chapter 3, we use GPS measurements of 16 continuous sites taken within 400 days of the Dusky Sound earthquake, supplemental campaign GPS data acquired during August 2009 and Differential Interferometric Synthetic Aperture Radar (DInSAR) observations to address the postseismic mechanisms following the mainshock.

We demonstrate that viscoelastic rebound models do not account for either observed short-term or southward displacement in the near-epicentral area (Section 3.3). Therefore, significant afterslip is required to entirely explain the postseismic displacement.

We resolve time-dependent afterslip models using PCAIM-inversion (Principal Component Analysis-based Inversion Method) framework (Section 3.2.3). To regularise the time-dependent inversion, we apply Laplacian smoothing and decompose the displacement signals into spatial and temporal functions. In contrast with other timedependent inversion techniques, this method does not require the introduction of additional temporal hyperparameters.

We account for the viscoelasticity of the lower crust and upper mantle by subtracting viscoelastic responses to coseismic stress changes from observed displacements. A Maxwell rheology with viscosities of $5 \times 10^{18}-10^{20} \mathrm{~Pa} \cdot \mathrm{s}$ and an elastic thickness of 45 $\mathrm{km}$ are assumed. We consider the viscoelastic responses to postseismic stress changes induced by afterslip throughout an iterative method (Figure 3.5). We demonstrate that afterslip models vary depending on the viscosity of the viscoelastic half-space. We therefore conclude that postseismic mechanisms and associated stress and strain models cannot be unique while the viscosity of the half-space is unknown.

Four options for slip-boundary constraints have been examined. In our final model, with no-slip boundary constraints on the sides and bottom of the fault, we estimate 
two main patches located on either sides of the coseismic slip patch (white contours in Figure 3.9) reaching to the ground surface and depth $80 \mathrm{~km}$.

Resolving a unique solution for the afterslip spatial modes depends on the density of ground measurements; The sparsity of the continuous GPS measurements in the central and southern South Island may cause ambiguity in our final postseismic model. There is also a lack of campaign GPS measurements spanning the early postseismic interval excluding the coseismic signal. Consequently, the misfit of the postseismic model with the campaign observations is mostly due to the uncertainty of the slip model used to remove the coseismic effects. The campaign measurements do not span the long-term postseismic interval between the Dusky Sound and the Darfield earthquakes. As a result, long-term spatial modes of the afterslip model are poorly constrained.

Strain and stress changes induced by the earthquake and corresponding postseismic mechanisms are calculated for the southern and central South Island (Section 3.4.3). Taking account of estimated postseismic mechanisms yields enlargement of the contractional and extensional strain vectors and expansion of the zones of positive coseismic Coulomb stress changes. In the Canterbury region, we estimate negative Coulomb stress changes on the Charing Cross Fault and a few kilopascals of positive Coulomb stress changes on the Greendale Fault. Principal contractional axis is calculated as $42^{\circ}$ clockwise around the secular contractional axis and $35^{\circ}$ clockwise around the previously determined $S_{\text {Hmax }}$. Principal contractional and extensional strains are $4 \%$ and $80 \%$ of the equivalent secular principal strains, respectively. Accordingly, we infer that Coulomb stress changes and strain rates induced by the earthquake and postseismic mechanisms are insignificant in the Canterbury region. Therefore, we cannot conclude that either the Dusky sound earthquake triggered or advanced the Canterbury earthquake sequence of 2010-2012.

\section{Further research}

The cGPS measurements are corrected for seasonal effects and mean secular velocities (Section 3.2.1). This dataset spans the time interval between the Dusky Sound and Darfield earthquakes for a period of approximately 400 days. There is a long-term campaign dataset available taken during February 2014. We did not constrain the long-term effect of postseismic mechanisms with this dataset. Extending the time interval for the cGPS data and constraining the postseismic models with this campaign measurement is recommended for further study. The uniqueness of the postseismic models can be improved considering the long-term effects of viscoelastic rebound. 


\subsection{Coupling afterslip and viscoelastic rebound}

In Chapter 4, we propose the Iterative Decoupling of Afterslip and Viscoelastic relaxation (IDAV) method to decouple the time-dependent postseismic displacement into elastic and viscoelastic space and time functions. We examine our framework with analytical and numerical sample data produced by the semi-analytical viscoelastic Green's functions and the Finite Element Method (FEM), respectively. We also apply this method to the observed postseismic displacement following the Dusky Sound earthquake assuming viscosities of $2 \times 10^{18}-4 \times 10^{18} \mathrm{~Pa} \cdot \mathrm{s}$. We demonstrate ambiguity in modelling the postseismic mechanisms in order to explain the Post-Dusky Sound displacement. Variation of viscosity values equal to or greater than $2 \times 10^{18} \mathrm{~Pa} \cdot \mathrm{s}$ yields different afterslip models.

\section{Contributions}

The main contribution of this chapter is adapting the PCAIM-inversion method to accommodate the viscoelastic Green's functions. The general form of the Sylvester equation (Equation 4.26; Section 4.2) is simplified with the assumptions that:

- One temporal mode suffices to decouple the viscoelastic Green's functions; and

- The spatial mode of the elastic displacement is obtained from unconstrained or constrained decomposition with imposing the temporal mode of the viscoelastic Green's functions.

\section{Further research}

We decompose the observed postseismic signals into two components of elastic and viscoelastic displacements. We do not consider temporally independent sources of displacement, which induce independent components in the observed signal. Adapting our inversion method to accommodate other techniques such as the Independent Component Analysis Inversion Method (ICAIM) is a topic of further study (Gualandi et al., 2015). Accordingly, ICAIM is able to separate sources that are temporally independent in observed signals.

We assess the IDAV method for use as an inversion framework for understanding postseismic transient afterslip. Potentially this method is applicable to the inversion of geodetic data to estimate the spatial and temporal evolution of Slow Slip Events (SSEs) 
in an elastic/viscoelastic medium.

The effect of fluid flow due to the coseismic stress changes induces a short-term displacement in the total observed signal (Peltzer et al., 1996, 1998). Using the IDAV method, the total displacement assumed to be the sum of viscoelastic response to coseismic changes and elastic and viscoelastic responses to afterslip. Therefore, corresponding displacement produced by poroelastic effects of earthquakes should be considered in future studies.

The IDAV method converges to a unique solution as a result of spatial Laplacian smoothing and decomposition. However, this method does not guarantee that the solution is physically significant. For instance, a viscosity of $10^{18} \mathrm{~Pa} \cdot \mathrm{s}$ as used in analysing the postseismic displacement following the Dusky Sound earthquake, yields a turning point in the estimated elastic time function. Other regularisation techniques of the Sylvester equation may ensure the physical significance of the solutions.

A unified inversion method considers the effects of viscoelastic and poroelastic rebound, the heterogeneity of lithospheric structure and other complexities such as an elasticslab geometry. This framework provides a more realistic understanding of the spatial and temporal evolution of seismic and aseismic slips and SSEs.

Our attempt in Chapter 4 requires more steps to be taken towards a unified framework for inversion of temporally dense geodetic observations. The ultimate aim of time-dependent inversion techniques is achieving an insight into controlling factors and interactions between various phenomena in a seismic cycle.

\subsection{Post-Canterbury displacement}

We experience difficulties in interpreting the sense of postseismic movement along and across the faults that ruptured during the Darfield earthquake. The TerraSAR-X (TSX) and COSMO-SkyMed (CSK) acquisitions on descending tracks are contaminated by major orbital and atmospheric artefacts. There is also a lack of campaign GPS measurements spanning the area encompassing the faults that ruptured during the Darfield earthquake. As a result, we cannot evaluate the sense of postseismic displacement using both InSAR and GPS data.

The frictional afterslip is computed as a first order approximation. Displacement profiles across the Greendale East Fault are obtained from the descending TSX and ascending CSK interferograms. Such additional analyses enable us to distinguish between 
the postseismic displacement processes in spite of the uncertainties described above. We conclude that poroelastic rebound and dilatancy recovery are the major postseismic mechanisms operating within approximately one month following the Darfield earthquake.

A dataset including ascending CSK acquisitions spans eastern end of the Greendale East Fault towards the western end of the Western Christchurch Fault in the time interval between the Darfield and Christchurch earthquakes. In this time interval, 12 acquisitions do not suffice to mitigate the long-wavelength artefacts completely in the region. Therefore, we cannot interpret the longer-term Darfield earthquake postseismic mechanisms and the corresponding stress transfer to the faults that ruptured during the Christchurch earthquake.

The dataset used following the Christchurch earthquake includes 66 descending TSX acquisitions. The estimated temporal displacement is affected by random variations of tropospheric perturbations. Stacking the interferograms mitigates the effects of random tropospheric artefacts in the estimated velocity. We observe and model a displacement gradient across the subsurface lineament extending from the Greendale East towards the Western Christchurch Faults. To explain this observation, we suggest afterslip on combinations of several faults: the lineament, Greendale East, NE Fault from epicentre and Western and Eastern Christchurch Faults. We cannot infer with certainty that the observed gradient is due to the aseismic slip on the lineament, although the lineament has been associated with a band of seismicity.

A subsidence signal is measured by this dataset in Christchurch and along the coast. We associate this signal to either dilatancy recovery on the faults that ruptured during the Christchurch earthquake and aftershocks in June and December and/or poroelastic rebound and/or hydrological effects of the earthquake such as liquefaction.

\section{Further research}

Fault damage and its recovery may justify the lack of afterslip following several strikeslip earthquakes (Fialko et al., 2005; Fielding et al., 2009) around the world such as the Darfield earthquake.

In Section 5.3.2, a regular grid of Mogi point sources is used to model the postseismic subsidence signal on the faults that ruptured during the Darfield earthquake. This model does not address coseismic and postseismic changes of seismic P- to S-wave velocity ratios due to the weakening of the crust. A physical method with which to 
estimate the displacement signal resulting from fault-damage is of great use.

\subsection{Unanswered questions from Section 1.3}

We provide answers to questions posed in Section 1.3 as the closing section of the thesis.

1. What can geodetic data reveal about postseismic displacement in response to multiple earthquakes?

(a) What circumstances or conditions are favourable in enhancing the effectiveness of InSAR for measuring low-strain rate tectonic deformations?

Measuring low-strain rate tectonic deformations using conventional InSAR techniques encounters several major limitations. Spatial and temporal decorrelation due to changes in scattering properties of the ground surface (Zebker and Villasenor, 1992), spatial and temporal variations of atmospheric effects and errors in satellite orbits and surface elevation often overwhelm the determination of crustal deformation (Zebker et al., 1997). Ferretti et al. $(2000,2001)$ tackle the problem of coherence loss due to decorrelation by identifying permanent scatterers in urban regions. In urban regions more permanent scatterers can be identified by measuring their amplitude scintillations. Hooper et al. (2004) used phase analysis in order to identify significant number of permanent scatterers in non-urban regions to measure the episodic volcanic deformation. This method is used in our displacement measurement in Section 5.4.1 to estimate the post-Christchurch displacement. Time and space variations of atmospheric water vapour lead to up to $10 \mathrm{~cm}$ errors in measuring ground displacement in wet regions such as Hawaii and potentially New Zealand. This effect is a major issue we encounter in our displacement measurement in the Canterbury region using TerraSAR-X acquisitions. The only way to mitigate this error is using multi passes of the same region (Zebker et al., 1997). Time series techniques by averaging independent interferograms and generating velocity maps significantly reduce the effect of atmospheric delays. Tropospheric variations of temperature and pressure are of smaller magnitudes and elevation-correlated (Zebker et al., 1997).

2. What mathematical framework can we use to provide unique solutions of displacement and stress evolution for postseismic time intervals? 
(a) How do we optimally choose rheology parameters in order to model the displacement and stress evolution?

(b) How can the uncertainty of input parameters for models such as fault geometry and slip distribution change the inferred stress changes?

(c) Is the inferred model of stress evolution unique?

The mathematical framework introduced in Chapter 4 is one step forward towards providing unique solutions of displacement and stress evolutions. This framework allows us to explain postseismic displacement signals by the coupled mechanism of afterslip and viscoelastic relaxation. Taking into account the effect of poroelasticity is left for further study. We have discussed the non-uniqueness of postseismic solutions in Section 2.2.1. We explained how to improve the uniqueness of postseismic solutions in terms of using other observations from sources such as tomography, thermo-kinematics models, seismicity, gravity signals observed by the GRACE mission and the local hydro-isostatic adjustment associated with the mantle flow. Assumptions made in Chapter 3 regarding the viscosity of the lower crust and upper mantle, the thickness of elastic layer and the geometry of subduction zone require more complementary (structural) information to provide unique solutions of postseismic mechanisms and stress models.

3. Can we assess the triggering effect of each earthquake on the later events by modelling the stress and strain evolution? Particularly, can we calculate the strain and stress evolution following the Dusky Sound earthquake and examine its effects on the Canterbury earthquake sequence of 2010-2012?

We have assessed the triggering effect of the Dusky Sound earthquake on the Canterbury earthquake sequence by means of accumulated strain and stress postseismic added to coseismic models (see Section 3.4.3). This method accounts for the static stress changes due to coseismic and postseismic mechanisms, which is limited to relatively smaller distances of a few $100 \mathrm{kms}$. 

Appendices 



\section{Appendix A}

\section{Coulomb stress changes}

The transformation matrix equation for a stress tensor from the geographic coordinate system $\left(S_{x y z}\right)$ to the fault-plane coordinate system $\left(S_{x^{\prime} y^{\prime} z^{\prime}}\right)$ is

$$
S_{x^{\prime} y^{\prime} z^{\prime}}=R S_{x y z} R^{\prime}
$$

where

$$
R=\left[\begin{array}{ccc}
1 & 0 & 0 \\
0 & -1 & 0 \\
0 & 0 & 1
\end{array}\right]\left[\begin{array}{ccc}
1 & 0 & 0 \\
0 & \cos (D) & \sin (D) \\
0 & -\sin (D) & \cos (D)
\end{array}\right]\left[\begin{array}{ccc}
\cos (S) & -\sin (S) & 0 \\
\sin (S) & \cos (S) & 0 \\
0 & 0 & 1
\end{array}\right]
$$

and

$$
S=s-90
$$

where $s$ and $D$ are the strike and dip angles of the fault (Figure A.1).

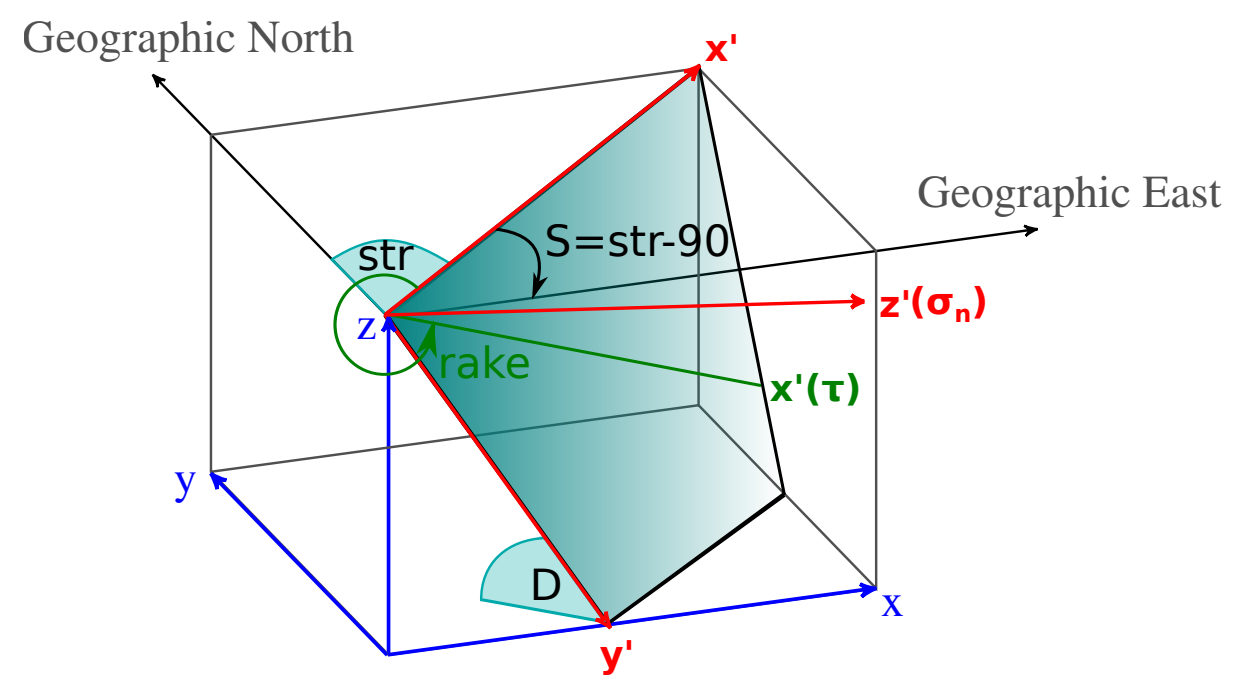

Figure A.1: Transformation from the geographic coordinate system to the fault-plane coordinate sytem

Normal $\left(\sigma_{n}\right)$ and shear stress $(\tau)$ components on the fault plane are obtained from the 
transformed stress tensor in the fault-plane coordinate system

$$
\begin{aligned}
\sigma_{n} & =S_{z^{\prime} z^{\prime}} \\
\tau & =\cos (r) S_{x^{\prime} z^{\prime}}-\sin (r) S_{y^{\prime} z^{\prime}}
\end{aligned}
$$

where $\mathrm{r}$ is rake angle and the transformed stress tensor is of the form

$$
S_{x^{\prime} y^{\prime} z^{\prime}}=\left[\begin{array}{ccc}
S_{x^{\prime} x^{\prime}} & S_{x^{\prime} y^{\prime}} & S_{x^{\prime} z^{\prime}} \\
S_{x^{\prime} y^{\prime}} & S_{y^{\prime} y^{\prime}} & S_{y^{\prime} z^{\prime}} \\
S_{x^{\prime} z^{\prime}} & S_{y^{\prime} z^{\prime}} & S_{z^{\prime} z^{\prime}}
\end{array}\right]
$$

Pore pressure $(P)$ is obtained from average of the normal stress components in the new coordinate system

$$
P=\left(S_{x^{\prime} x^{\prime}}+S_{y^{\prime} y^{\prime}}+S_{z^{\prime} z^{\prime}}\right) / 3
$$

therefore, Coulomb stress change is expressed as

$$
\Delta C f f=\tau+f\left(\sigma_{n}-B P\right)
$$

where $f$ and $B$ are fault friction and Skempton's coefficients, respectively. All the variables are summarised in Table A.1.

Table A.1: Table of nomenclature

\begin{tabular}{|c|c|}
\hline$D$ & Dip \\
\hline$s$ & Strike \\
\hline$S$ & str -90 \\
\hline$S_{x y z}$ & Cartesian stress tensor \\
\hline$S_{x^{\prime} y^{\prime} z^{\prime}}$ & Transformed stress tensor \\
\hline$R$ & Rotation matrix \\
\hline$r$ & Rake \\
\hline$\sigma_{n}$ & Normal stress \\
\hline$\tau$ & Shear stress \\
\hline$f$ & Fault friction coefficient \\
\hline$B$ & Skempton's coefficient \\
\hline$P$ & Pressure \\
\hline$\Delta C f f$ & Coulomb stress changes \\
\hline
\end{tabular}




\section{Appendix B}

\section{Global compilation of viscosity, rheology and depth of the top viscoelastic layer}

For each row of Table B.1, we summarise the metadata of the earthquakes. In Table B.2, for each row we mention rheology, vertical or lateral heterogeneities and viscosity value for each layer. Regarding the rheology field, "M" stands for Maxwell, "B" Burger's body, "P" Power-law, "SLS" Standard Linear Solid and "NN" is the general term Non-Newtonian fluid. Regarding the viscosity field, layers are separated with " " symbol, multiple viscosities for each layer with "," and the range of viscosity in a parenthesis "()". Table B.3 is the summary of the thickness and depth of each layer. "SL" stands for Slab and "UP" Upper Plate thickness in case of subduction earthquakes. "LC", "UM" and " $H S$ " stand for depths associated with Lower Crust, Upper Mantle and Half-Space for non-subduction zone earthquakes. 


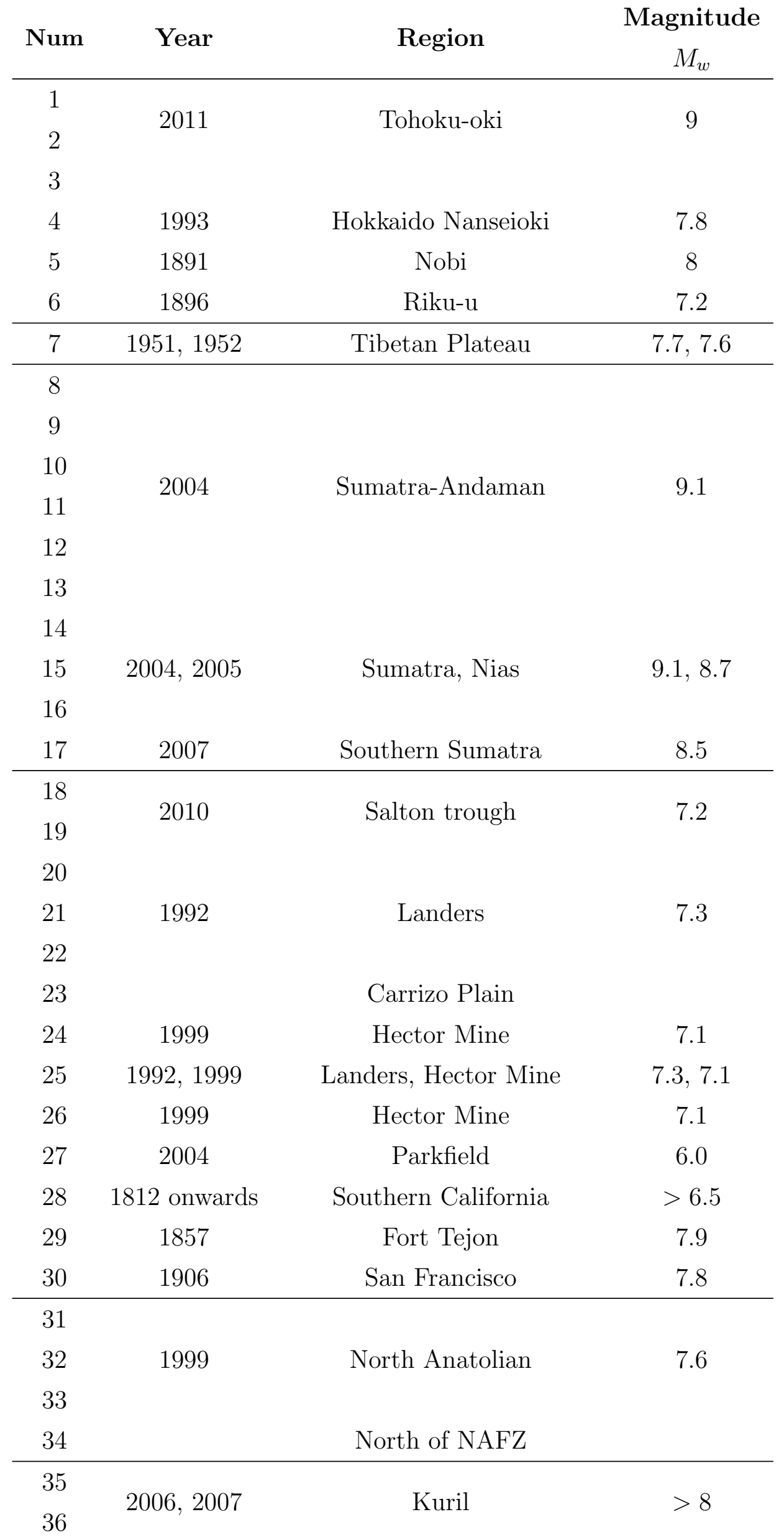




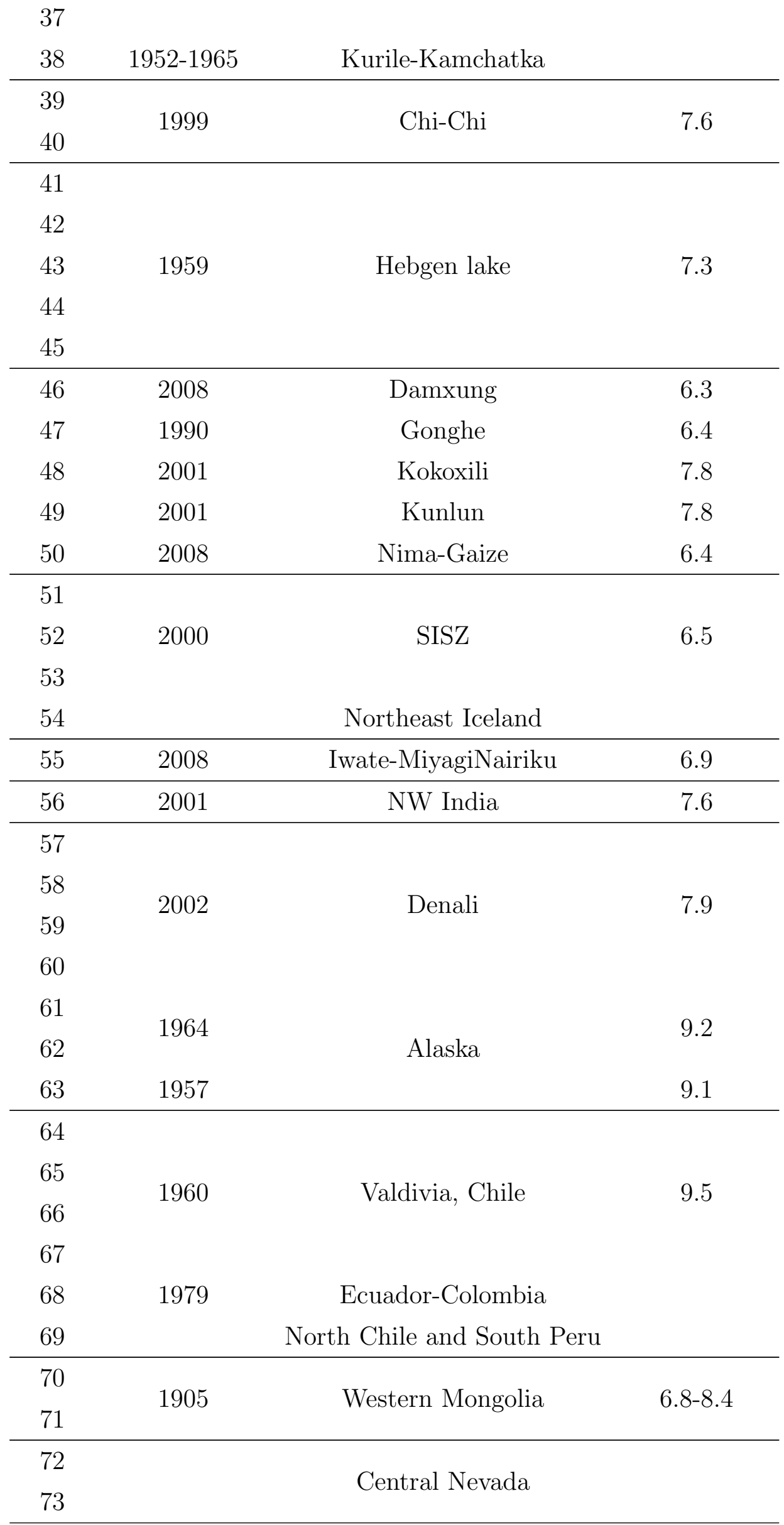




\begin{tabular}{|c|c|c|c|}
\hline $\begin{array}{l}74 \\
75\end{array}$ & 1997 & Umbria-Marche, Italy & 6.1 \\
\hline $\begin{array}{l}76 \\
77\end{array}$ & 1980 & Irpinia & 6.89 \\
\hline 78 & & Northern Caribbean plate & \\
\hline 79 & 1974 & Southeastern caribbean & 6.1 \\
\hline $\begin{array}{l}80 \\
81\end{array}$ & 1989 & Loma Prieta & 6.9 \\
\hline 82 & 1991 & Racha Georgia & 7.15 \\
\hline 83 & 1981 & Southern Iran & $>6.4$ \\
\hline $\begin{array}{l}84 \\
85\end{array}$ & 1923 & Kanto & 7.9 \\
\hline 86 & 2001 & Southern Peru & 8.4 \\
\hline 87 & 1995 & Suez-Sinai, Jordan & 7.2 \\
\hline 88 & $1811-1812$ & New Madrid & \\
\hline
\end{tabular}

Table B.1: Global rheology database 


\begin{tabular}{|c|c|c|c|c|}
\hline \multirow{2}{*}{ Num } & \multirow{2}{*}{ Rheology } & \multicolumn{2}{|c|}{ Layering } & \multirow{2}{*}{$\begin{array}{l}\text { Viscosity }{ }^{1} \\
\left(10^{18} \text { Pas }\right)\end{array}$} \\
\hline & & Vertical & Lateral & \\
\hline 1 & B & 2 & No & $0.5,10$ \\
\hline 2 & M & 2 & No & 9 \\
\hline 3 & $\mathrm{P}$ & & Yes & $10-10^{4}-10^{2}-10^{3}$ \\
\hline 4 & M & 3 & No & 4 \\
\hline 5 & M & 3 & No & 20 \\
\hline 6 & M & 2 & No & 9.3 \\
\hline 7 & M & 2 & No & $(60-100)$ \\
\hline 8 & M & 2 & No & 8 \\
\hline 9 & B-M & $4^{2}$ & No & $1,10-10^{2}-10^{3}$ \\
\hline 10 & $\mathrm{P}-\mathrm{M}$ & $4^{3}$ & No & $((3-5)-10)-10^{2}-10^{3}$ \\
\hline 11 & B-M & & No & $3 \times 10^{3}-10$ \\
\hline 12 & B & $2^{4}$ & Yes $^{5}$ & $0.5,(10-50)$ \\
\hline 13 & M & 2 & No & 0.5 \\
\hline 14 & $\mathrm{~B}-\mathrm{M}$ & $4^{6}$ & No & $0.4,8-8-8 \times 10^{2}$ \\
\hline 15 & B-M & $4^{7}$ & No & $0.5,10-10^{2}-10^{3}$ \\
\hline 16 & M & $2^{8}$ & No & 35 \\
\hline 17 & M & 2 & No & 2.5 \\
\hline 18 & $\begin{array}{c}\mathrm{M}-\mathrm{M} \\
\mathrm{M}-\mathrm{P}-\mathrm{M}\end{array}$ & 3 & No & $\begin{array}{c}3-\sim 1 \\
3-P n=3.5-\sim 1\end{array}$ \\
\hline 19 & M-B & 3 & Yes $^{9}$ & $32-0.12,3.2$ \\
\hline 20 & M & 4 & No & $\sim\left(10-10^{2}\right)-\sim\left(10^{2}-10^{4}\right)-\sim(1-10)$ \\
\hline 21 & SLS-M-M & 4 & No & $16-8-2.7$ \\
\hline 22 & Biviscous $^{10}$ & & & $\left((3-4) \times 10^{-3} 11\right)$ \\
\hline 23 & M & 2 & Yes & $\left((0.1-2.9) \times 10^{2}\right)$ \\
\hline 24 & M-B & 3 & No & $32-0.16,4.6$ \\
\hline 25 & M-B & 3 & Yes & $\begin{array}{l}S W: 2 \times 10^{2}-2,10 \\
N E: 2 \times 10^{2}-4,40\end{array}$ \\
\hline 26 & $\mathrm{P}$ & & Yes & $\left(50-10^{3}\right)$ \\
\hline
\end{tabular}

\footnotetext{
${ }^{1}$ Values for multi-viscous rheology are separated by ",", for stratified layers by " -" and a single viscosity range by "()"

${ }^{2}$ High viscosity for the depth greater than $210 \mathrm{~km}$

${ }^{3}$ High viscosity for the depth greater than $210 \mathrm{~km}$

${ }^{4}$ We skip two other layers UM2 and LM

${ }^{5}$ The heterogeneity induced by the slab geometry

${ }^{6}$ Combined with afterslip

${ }^{7}$ Combined with afterslip

${ }^{8}$ We skip two other layers UM2 and LM

${ }^{9}$ Viscous lateral heterogeneity consistent with seismic structure

${ }^{10}$ Composite lower crust

${ }^{11}$ transient creep within 4-34 days after Landers earthquake
} 


\begin{tabular}{|c|c|c|c|c|}
\hline 27 & M & & No & 1 \\
\hline 28 & M & & No & $36-12$ \\
\hline 29 & M & 2 & No & $\sim 4$ \\
\hline 30 & $\mathrm{M}$ & 3 & No & $5-95$ \\
\hline 31 & $\mathrm{M}$ & 2 & Yes & $\left(\sim 1-10^{2}\right)$ \\
\hline 32 & SLS-M & 2 & No & $\sim 2-70$ \\
\hline 33 & $\mathrm{~B}$ & 2 & No & $(20-50)-2 \times 10^{2}$ \\
\hline 34 & M & 3 & No & $>5 \times 10^{2}$ \\
\hline 35 & $\mathrm{P}^{12}$ & 2 & Yes $^{13}$ & $0.2,1$ \\
\hline 36 & M & 2 & $\mathrm{Yes}^{14}$ & $(0.5-1)^{15}$ \\
\hline 37 & $\mathrm{~B}$ & 2 & No & 0.3 \\
\hline 38 & M & 2 & No & 0.5 \\
\hline 39 & M & $3^{16}$ & $\mathrm{Yes}^{17}$ & $0.5-(5-10)$ \\
\hline 40 & $\mathrm{NN}$ & 3 & No & $0.5-5 \times 10^{5}$ \\
\hline 41 & $\mathrm{M}$ & 3 & Yes & $\begin{array}{c}18 \sim 20-\sim 5 \\
19 \sim 3 \times 10^{3}-\sim 20\end{array}$ \\
\hline 42 & M & 2 & No & 4 \\
\hline 43 & $\mathrm{M}$ & 2 & No & 4 \\
\hline 44 & M & 2 & No & $\sim 10$ \\
\hline 45 & M & 2 & No & $2 \times 10^{3}$ \\
\hline 46 & $\mathrm{M}$ & 2 & No & 1 \\
\hline 47 & $\mathrm{M}$ & 2 & No & 90 \\
\hline 48 & M & 2 & No & 20 \\
\hline 49 & M & 2 & No & 1 \\
\hline 50 & M & 2 & No & 0.3 \\
\hline 51 & SLS & 2 & No & $(1-3)$ \\
\hline 52 & $\mathrm{M}-\mathrm{B}$ & 3 & No & $(5-10)-\sim 3$ \\
\hline 53 & M & 3 & No & $\sim 10-\sim 1-(3-4)$ \\
\hline 54 & M & 3 & No & $30-3$ \\
\hline 55 & $\mathrm{M}$ & 2 & No & $(2.4-4.8)$ \\
\hline 56 & $\mathrm{P}^{20}$ & 2 & No & $3-20$ \\
\hline
\end{tabular}

${ }^{12}$ The Maxwell rheology has been used with varying viscosity from 0.2 to 1 in four years which could be assumed equivalent to power-law viscosity

${ }^{13}$ Low viscosity mantle above the slab

${ }^{14}$ Low viscosity mantle above the slab

${ }^{15}$ Smaller than several subduction zones

${ }^{16}$ mid-crust and lower-crust viscoelastic layers

${ }^{17}$ Low viscosity region below the Central Range due to high temperature and rapid advection coincident with low seismic velocity and low $V_{p} / V_{s}$ ratio.

${ }^{18}$ Southeast of the Hebgen Lake fault

${ }^{19}$ Central and north-western Hebgen Lake fault

${ }^{20}$ Systematic increase of viscosity within six months 


\begin{tabular}{|c|c|c|c|c|}
\hline 57 & M & 3 & No & $10-1$ \\
\hline 58 & M & 2 & Yes & $(10-(3-4))$ \\
\hline 59 & M-B & 3 & No & $20-0.1,2.8$ \\
\hline 60 & M & 2 & No & $(6-15)$ \\
\hline 61 & M & 2 & No & $(23-39)$ \\
\hline 62 & M & 2 & No & $(12-22)$ \\
\hline 63 & $\mathrm{M}$ & 2 & No & $(12-22)$ \\
\hline 64 & M & 2 & No & $10^{2}$ \\
\hline 65 & M & 2 & No & 30 \\
\hline 66 & M & 2 & No & $\left(80-10^{2}\right)$ \\
\hline 67 & M & 2 & No & 25 \\
\hline 68 & M & 3 & No & $10-10^{3}$ \\
\hline 69 & M & 2 & No & 10 \\
\hline 70 & $\mathrm{M}$ & 3 & No & $3 \times 10^{-2}-0.2-(1-4)$ \\
\hline 71 & M & 2 & No & 3 \\
\hline 72 & M & 3 & No & $(5-50)-N A$ \\
\hline 73 & $\mathrm{M}$ & 3 & No & $10^{2.5}-10^{2}$ \\
\hline 74 & M & 4 & No & $1-(0.1-1)-10^{3}$ \\
\hline 75 & M & 4 & No & $10-1-10^{3}$ \\
\hline 76 & M & 2 & No & 10 \\
\hline 77 & M & 6 & No & 6 \\
\hline 78 & M & 3 & No & $30-10$ \\
\hline 79 & M & 2 & No & $10^{2}$ \\
\hline 80 & M & 2 & No & 0.3 \\
\hline 81 & M & 3 & No & $14-\infty$ \\
\hline 82 & M & 2 & No & 0.9 \\
\hline 83 & M & 3 & No & $1-10^{2}\left(10^{2}-10\right)$ \\
\hline 84 & M & 2 & Yes & 14,20 \\
\hline 85 & M & 3 & No & 5 \\
\hline 86 & $\mathrm{P}$ & 2 & Yes & $2.3 \times 10^{-2}, 0.11$ \\
\hline 87 & M & 3 & Yes & 1 \\
\hline 88 & & 4 & No & $\left.(0.25-1.9) \times 10^{2}-\infty-(2.5-19)\right)$ \\
\hline
\end{tabular}

Table B.2: Continuation of global rheology database: Viscoelastic models and viscosity values 


\begin{tabular}{|c|c|c|c|c|c|c|}
\hline \multirow{2}{*}{ Num } & \multicolumn{5}{|c|}{ Layers (km) } & \multirow{2}{*}{ Others } \\
\hline & $\mathrm{SL}^{21}$ & $\mathrm{UP}^{22}$ & $\mathrm{LC}^{23}$ & $\mathrm{UM}^{24}$ & $\mathrm{HS}^{25}$ & \\
\hline 1 & 30 & 30 & & & & Sun and Wang (2015) \\
\hline 2 & & & & & 50 & Yamagiwa et al. (2015) \\
\hline 3 & & & & & & Muto et al. (2013) \\
\hline 4 & & & 40 & & 90 & Ueda et al. (2003) \\
\hline 5 & & & 33 & & 163 & Pollitz and Sacks (1994) \\
\hline 6 & & & & & 30 & Suito and Hirahara (1999) \\
\hline 7 & & & & & 15 & Ryder et al. (2014) \\
\hline 8 & & & & & 65 & Gunawan et al. (2014) \\
\hline 9 & & & & & 40 & Hoechner et al. (2011) \\
\hline 10 & & & & & 60 & Reddy et al. (2010) \\
\hline 11 & 30 & 50 & & & & Pollitz et al. (2008) \\
\hline 12 & & & & & 62 & Han et al. (2008) \\
\hline 13 & & & & & 70 & Paul et al. (2007) \\
\hline 14 & & & & & 60 & Panet et al. (2010) \\
\hline 15 & & & & & 65 & Pollitz et al. (2006b) \\
\hline 16 & & & & & 62 & Reddy et al. (2012) \\
\hline 17 & & & & & 65 & Lubis et al. (2012) \\
\hline 18 & & & 10 & 22.5 & $\sim 40$ & Rollins et al. (2015) \\
\hline 19 & & & 15 & 30 & 60 & Johnson et al. (2007) \\
\hline 20 & & & 15 & & 30 & Pollitz et al. (2012) \\
\hline 21 & & & 16 & 30 & 50 & Pollitz et al. (2000) \\
\hline 22 & & & & & & Ivins (1996) \\
\hline 23 & & & & & $\sim(44-100)$ & Johnson and Segall (2004) \\
\hline 24 & & & 16 & & 30 & Pollitz (2003b) \\
\hline 25 & & & & & 43 & Pollitz (2015) \\
\hline 26 & & & & & 50 & Freed et al. (2012) \\
\hline 27 & & & & & 20 & Bruhat et al. (2011) \\
\hline 28 & & & & & & Freed et al. (2007) \\
\hline 29 & & & & & 16 & Rydelek and Sacks (2001) \\
\hline 30 & & & 11 & & 25 & Kenner and Segall (1999) \\
\hline
\end{tabular}

\footnotetext{
${ }^{21}$ Slab thickness

${ }^{22}$ Upper plate thickness

${ }^{23}$ Depth of Lower crust

${ }^{24}$ Depth of upper mantle

${ }^{25}$ Depth of half-space
} 


\begin{tabular}{|c|c|c|c|c|c|c|}
\hline 31 & & & & & 12 & Yamasaki et al. (2014) \\
\hline 32 & & & 20 & & 35 & Wang et al. (2009) \\
\hline 33 & & & 22 & & 32 & Hearn et al. (2009) \\
\hline 34 & & & 25 & & 30 & Hearn et al. (2002b) \\
\hline 35 & 62 & 62 & & & & Kogan et al. (2013) \\
\hline 36 & & & & & 62 & Kogan et al. (2011) \\
\hline 37 & & & & & & Vladimirova et al. (2011) \\
\hline 38 & & & & & 62 & Pollitz et al. (1998a) \\
\hline 39 & & & & & 30 & Rousset et al. (2012a) \\
\hline 40 & & & 22 & & 30.25 & Sheu and Shieh (2004) \\
\hline \multirow{2}{*}{41} & & & 17 & & 26 & \multirow{2}{*}{ Chang et al. (2013) } \\
\hline & & & 18 & & 31 & \\
\hline 42 & & & & & 38 & Nishimura and Thatcher (2003) \\
\hline 43 & & & & & 38 & Holdahl and Dzurisin (1991) \\
\hline 44 & & & & & $\sim 30-40$ & Reilinger (1986) \\
\hline 45 & & & & & 20 & Koseluk and Bischke (1981) \\
\hline 46 & & & & & 15 & Bie et al. (2014) \\
\hline 47 & & & & & 20.5 & Hao et al. (2012) \\
\hline 48 & & & & & 15 & Wen et al. $(2012 b)$ \\
\hline 49 & & & & & 32 & Diao et al. (2011) \\
\hline 50 & & & & & 14 & Ryder et al. (2010a) \\
\hline 51 & & & & & 15 & Decriem and Árnadóttir (2012) \\
\hline 52 & & & 10 & & 20 & Árnadóttir et al. (2005) \\
\hline 53 & & & 10 & $\sim(25-30)$ & 30 & Jónsson (2008) \\
\hline 54 & & & 12 & & 20 & Pollitz and Sacks (1996) \\
\hline 55 & & & & & $(19-23.5)$ & Ohzono et al. (2012) \\
\hline 56 & & & & & 34 & Chandrasekhar et al. (2009) \\
\hline 57 & & & 12 & & $30 N, 45 S$ & Biggs et al. (2009) \\
\hline 58 & & & & & 50 & Freed et al. (2006) \\
\hline 59 & & & $\sim 15$ & & 38 & Pollitz (2005) \\
\hline 60 & & & & & $45-85$ & Johnson et al. (2009) \\
\hline 61 & 90 & 30 & & & & Suito and Freymueller (2009) \\
\hline 62 & & & 20 & & 80 & Wahr and Wyss (1980) \\
\hline 63 & & & 50 & & 200 & Wahr and Wyss (1980) \\
\hline 64 & & & & & 46 & Lorenzo-Martín et al. (2006) \\
\hline 65 & 30 & 40 & & & & Khazaradze et al. (2002) \\
\hline
\end{tabular}




\begin{tabular}{|c|c|c|c|c|c|c|}
\hline 66 & & & & & 18 & Piersanti (1999) \\
\hline 67 & 30 & 40 & & & & Hu et al. (2004) \\
\hline 68 & & & 40 & & 150 & White et al. (2003) \\
\hline 69 & & & & & 80 & Casarotti and Piersanti (2003) \\
\hline 70 & & & 35 & & 50 & Vergnolle et al. (2003) \\
\hline 71 & & & & & 30 & Calais et al. (2002) \\
\hline 72 & & & 15 & & 30 & Hetland and Hager (2003) \\
\hline 73 & & & & & & Hammond et al. (2009) \\
\hline 74 & & & 20 & & 35 & Aoudia et al. (2003) \\
\hline 75 & & 10 & 20 & & 35 & Riva et al. (2000) \\
\hline 76 & & & 20 & & & Dalla Via et al. (2005) \\
\hline 77 & & & & & 18.5 & Dalla Via et al. (2003) \\
\hline 78 & & & 16 & & 33 & Pollitz and Dixon (1998) \\
\hline 79 & & & & & 35 & Kozuch et al. (1996) \\
\hline 80 & & & & & & Linker and Rice (1997) \\
\hline 81 & & 16 & & & 30 & Pollitz et al. (1998b) \\
\hline 82 & & & 25 & 45 & & Podgorski et al. (2007) \\
\hline 83 & & & $\sim 15$ & & $\sim 30$ & Podgorski et al. (2007) \\
\hline 84 & & & & & 50 & Pollitz et al. (2006c) \\
\hline 85 & & & & & 30 & Matsu'ura and Iwasaki (1983) \\
\hline 86 & & $45-65$ & & & & Hergert and Heidbach (2006) \\
\hline 87 & & & 15 & & 30 & Piersanti et al. (2001) \\
\hline 88 & & & 16 & 33 & 50 & Rydelek and Pollitz (1994) \\
\hline
\end{tabular}

Table B.3: Continuation of global rheology database: Range of layers in depths 


\section{Appendix $\mathrm{C}$}

\section{Post-Dusky Sound supplementary files}

Here we present cGPS displacement observations (blue lines) versus calculated afterslip coupled with viscoelastic rebound using the IDAV method (red lines) for a Maxwell rheology of viscosity $2 \times 10^{18} \mathrm{~Pa} \cdot \mathrm{s}$ (See Section 4.4). 

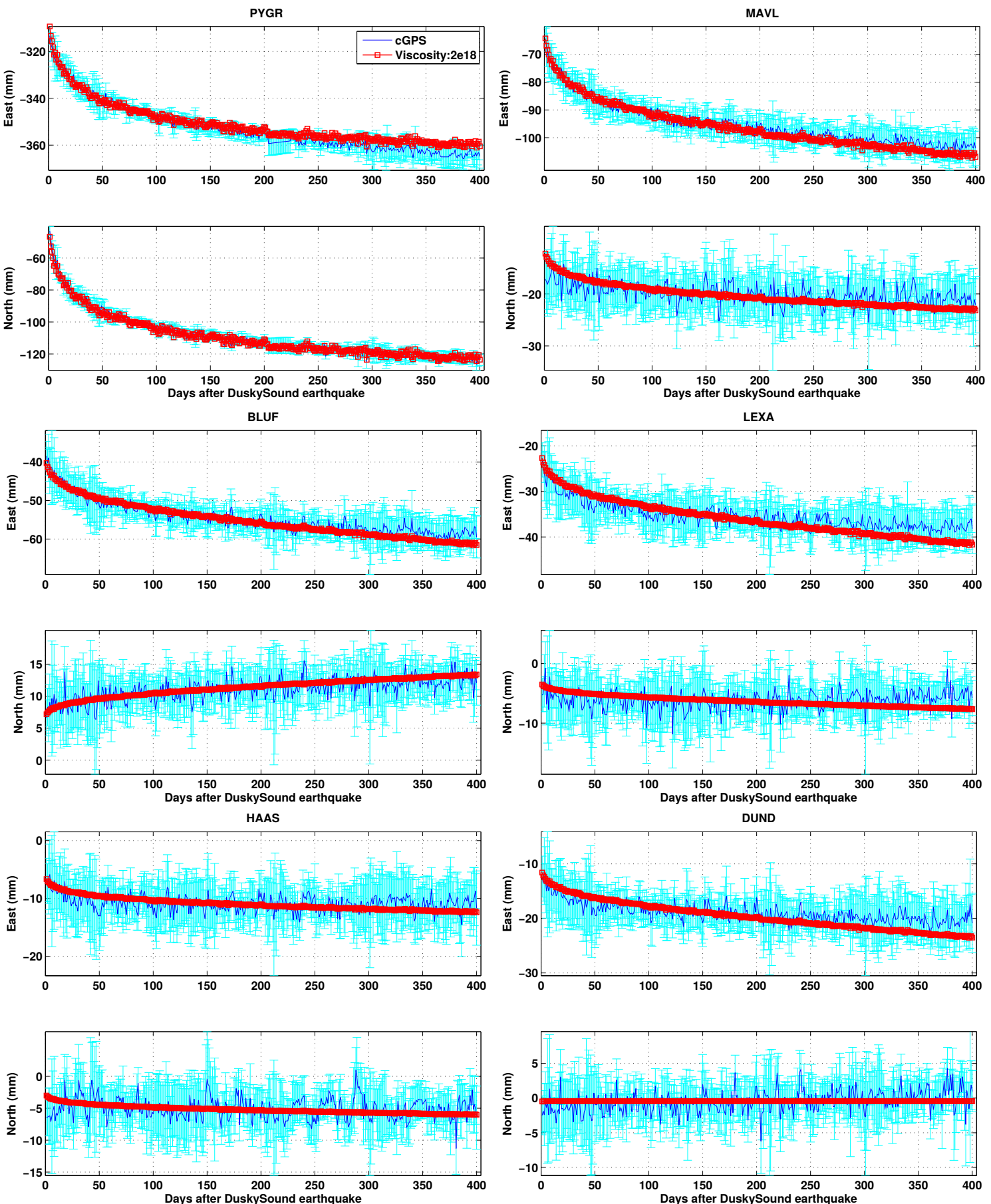

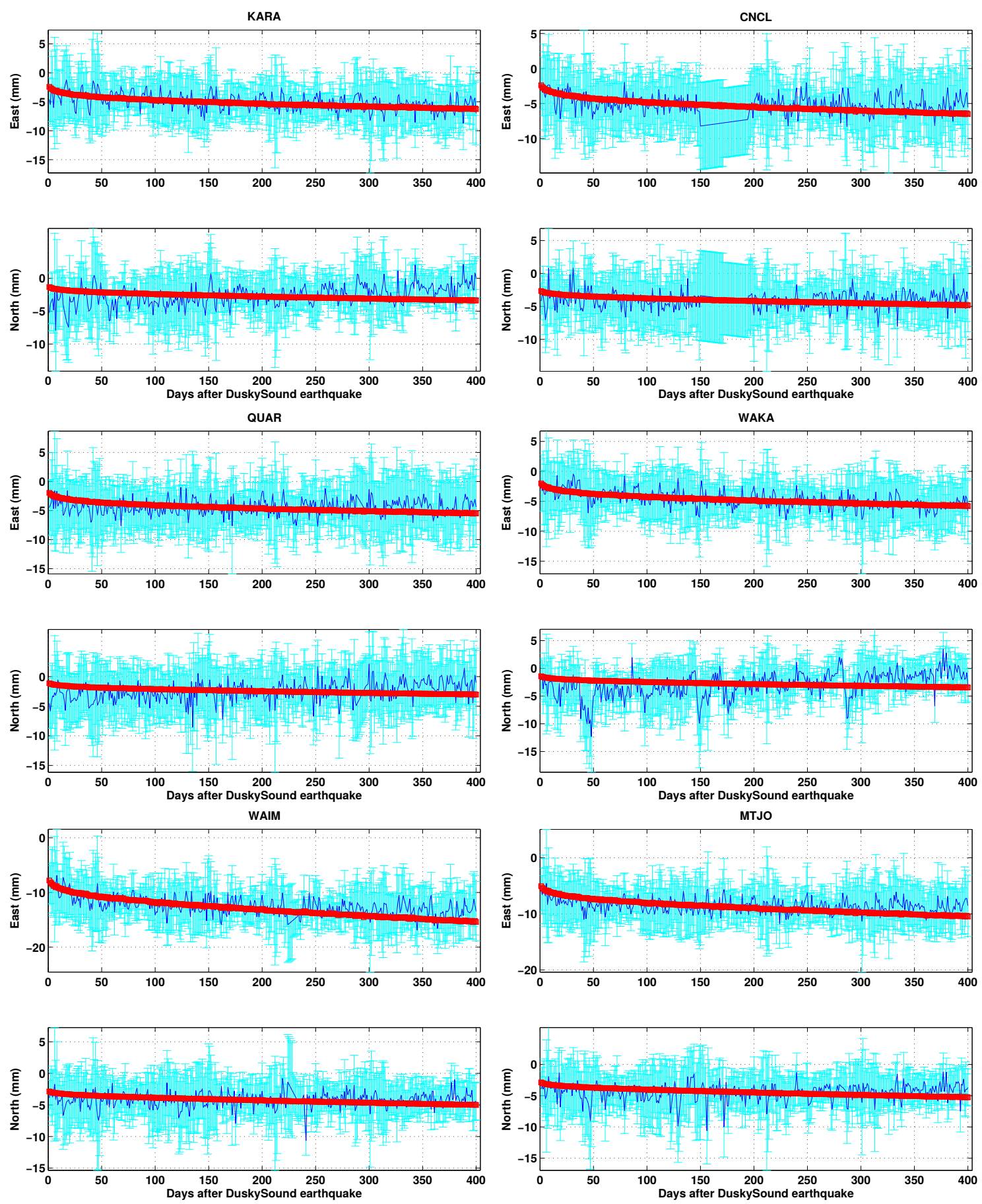

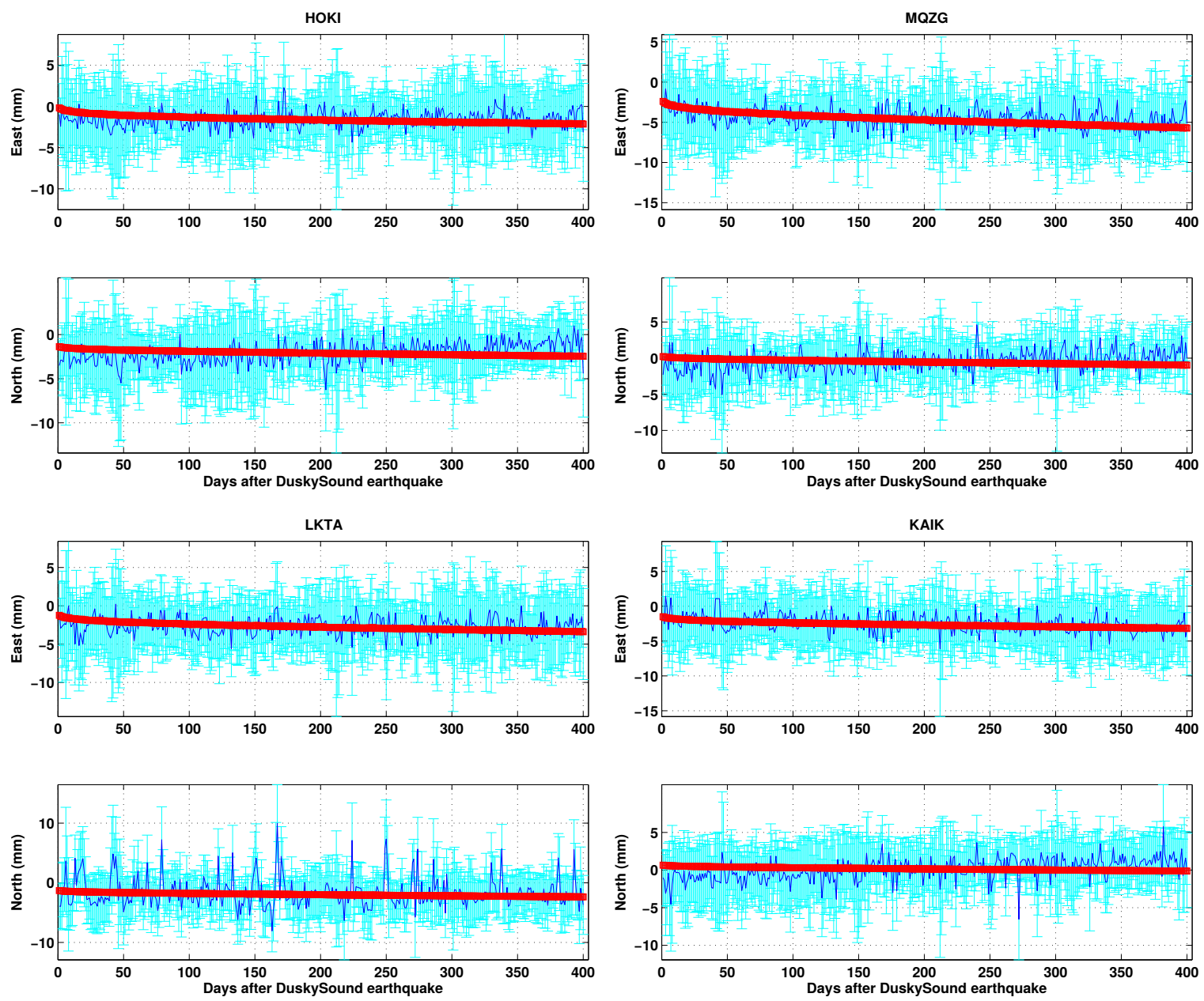

Figure C.1: cGPS displacement observations (blue lines) and estimated afterslip coupled with viscoelastic rebound (red lines) for a Maxwell rheology of viscosity $2 \times 10^{18} \mathrm{~Pa} \cdot \mathrm{s}$. 


\section{Appendix D}

\section{Post-Canterbury supplementary files}

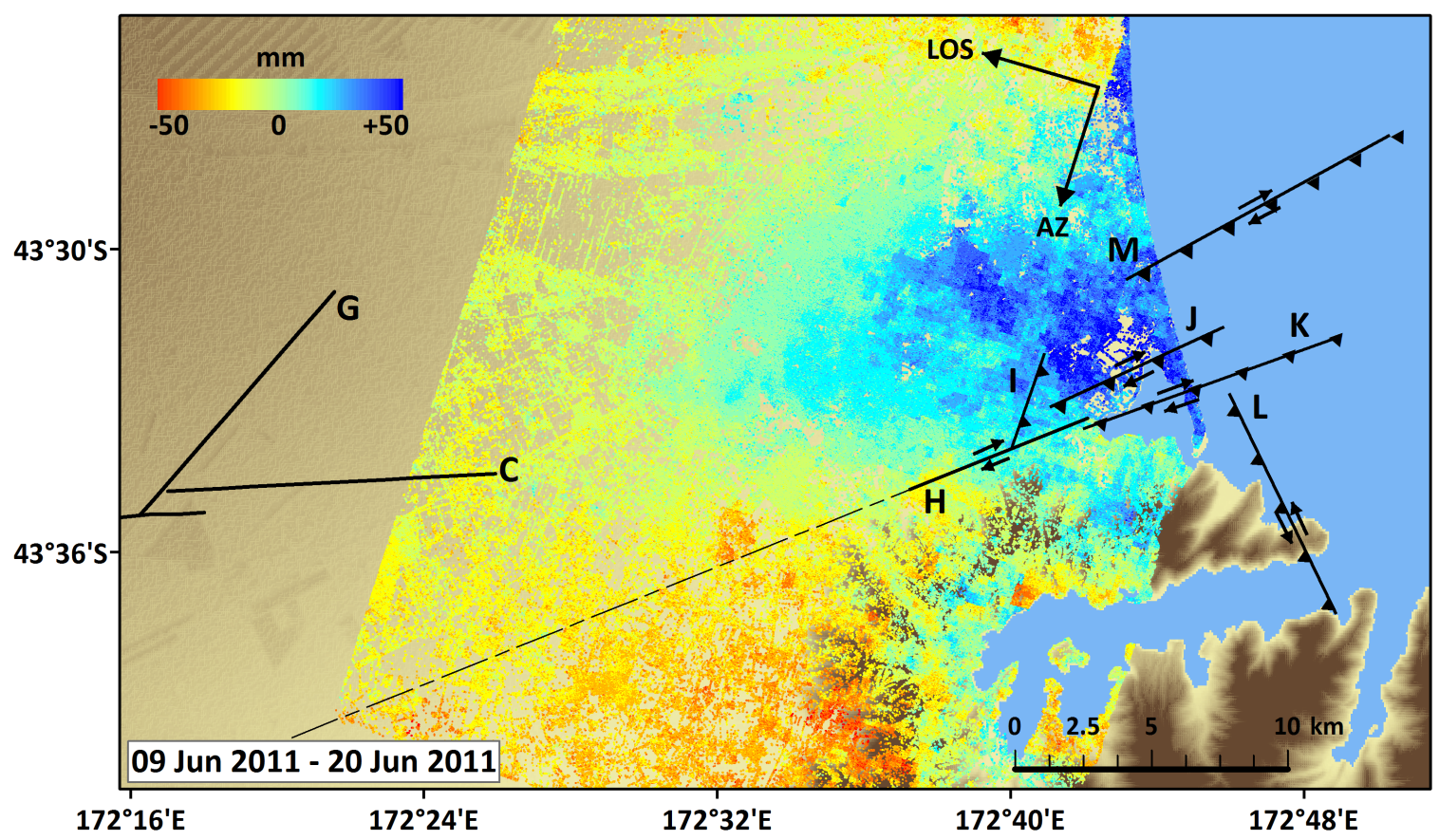

Figure D.1: Coseismic displacement due to the 13 June 2011 earthquake in Christchurch acquired from two descending TSX acquisions between the $9^{\text {th }}$ and $20^{\text {th }}$ of June 2011. Red colours indicate movement away from satellite and blue colours indicate movement towards the satellite. 


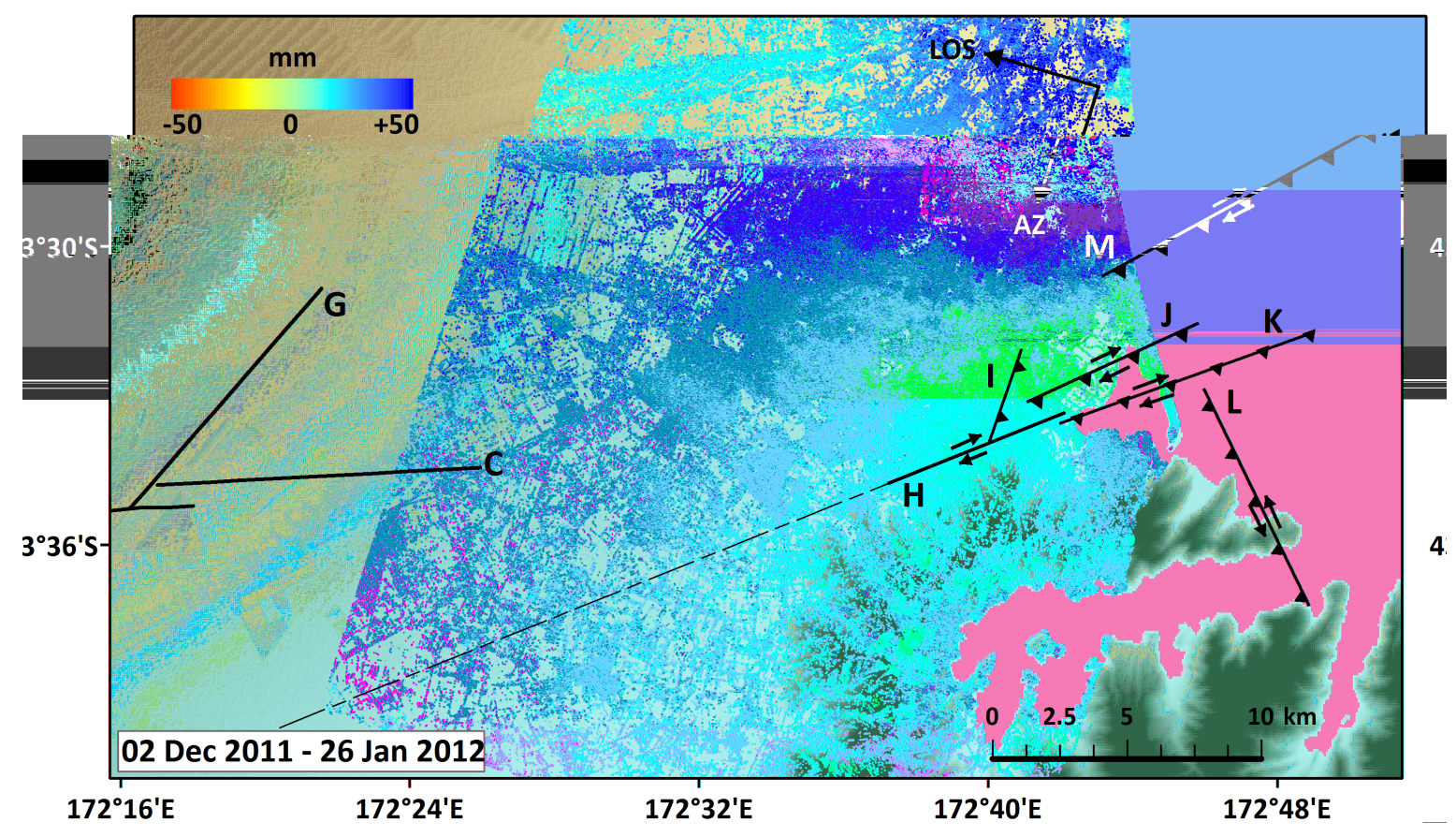

Figure D.2: Coseismic displacement due to the 23 December 2011 earthquake in Christchurch acquired from two descending TSX acquisions between the $9^{\text {th }}$ and $20^{\text {th }}$ of June June 2011. Red colours indicate movement away from satellite and blue colours indicate movement towards the satellite.

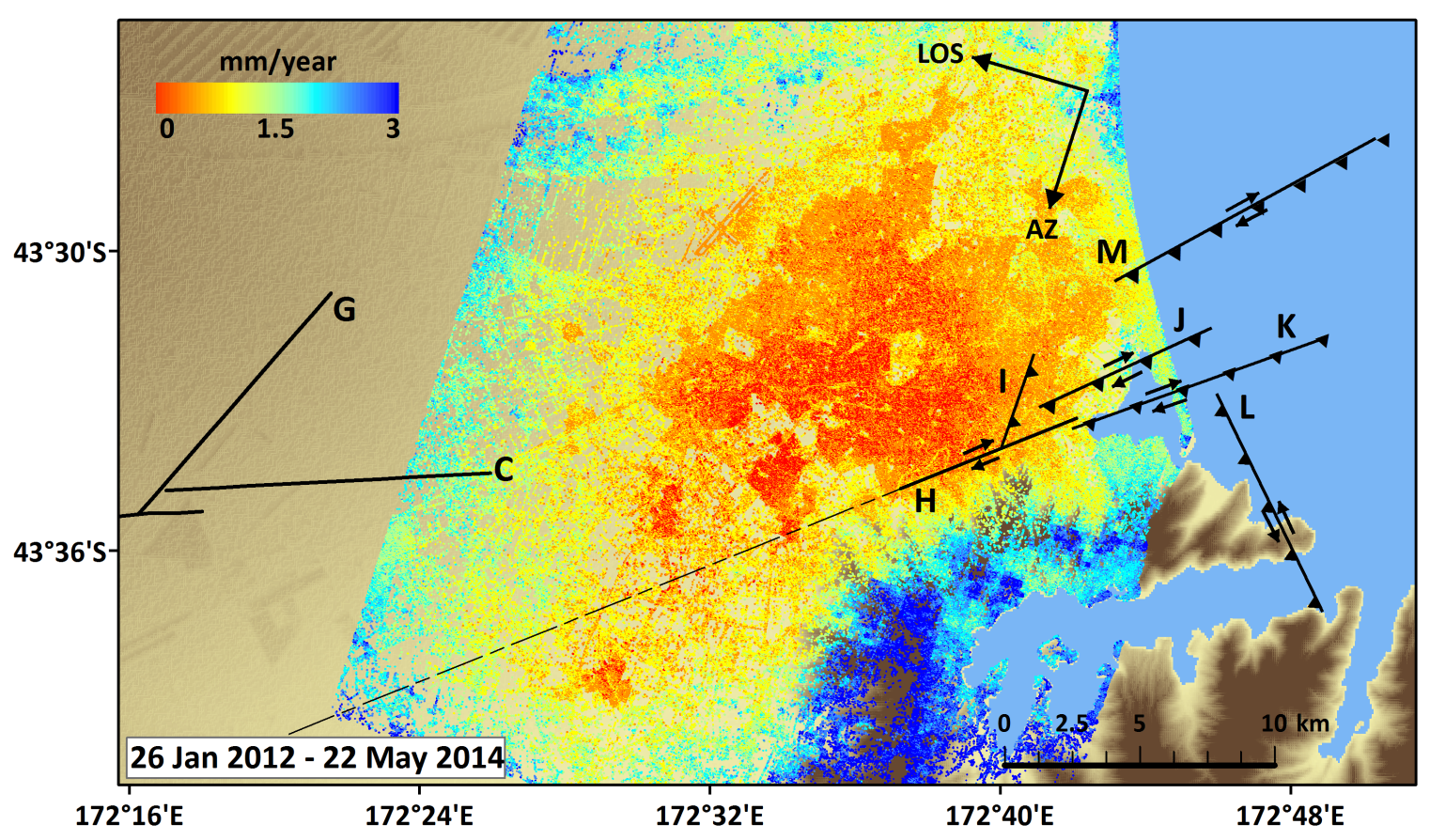

Figure D.3: Standard deviation of mean line of sight velocity acquired from TSX acquisions between 26 Januar 2012 and 22 May 2014. Larger values of standard deviation is due to the stratified atmospheric error over the area on the south-eastern corner of the case study with high gradient of elevation. We discard this area in our calculations. Red colours indicate movement away from satellite and blue colours indicate movement towards the satellite. 


\section{Bibliography}

Aagaard, B., Kientz, S., Knepley, M., Strand, L., and Williams, C. (2015). PyLith User Manual, Version 2.1.0.

Aagaard, B., Knepley, M., and Williams, C. (2013). A domain decomposition approach to implementing fault slip in finite-element models of quasi-static and dynamic crustal deformation. Journal of Geophysical Research: Solid Earth.

Aagaard, B., Williams, C., Knepley, M., Kientz, S., and Strand, L. (2009). PyLith.

Ackroyd, R. (1986). Generalized least squares as a generator of variational principles and weighted residual methods for FEM transport methods. Progress in Nuclear Energy, 18(1):45-62.

Akaike, H. (1977). On entropy maximization principle. Application of statistics.

Akaike, H. (1980). Likelihood and the Bayes procedure. Trabajos de estadística y de investigación operativa, 31(1):143-166.

Aki, K. and Richards, P. (1980). Quantative seismology: Theory and methods. Vol .

Altamimi, Z., Métivier, L., and Collilieux, X. (2012). ITRF2008 plate motion model. Journal of Geophysical Research: Solid Earth (1978-2012), 117(B7).

Anderson, B. D. and Moore, J. B. (2012). Optimal filtering. Courier Corporation.

Anderson, H., Webb, T., and Jackson, J. (1993). Focal mechanisms of large earthquakes in the South Island of New Zealand: implications for the accommodation of PacificAustralia plate motion. Geophysical journal international, 115(3):1032-1054.

Aoudia, A., Borghi, A., Riva, R., Barzaghi, R., Ambrosius, B., Sabadini, R., Vermeersen, L., and Panza, G. (2003). Postseismic deformation following the 1997 Umbria-Marche (Italy) moderate normal faulting earthquakes. Geophysical research letters, 30(7).

Árnadóttir, T., Jónsson, S., Pollitz, F. F., Jiang, W., and Feigl, K. L. (2005). Postseismic deformation following the June 2000 earthquake sequence in the south Iceland seismic zone. Journal of Geophysical Research: Solid Earth (1978-2012), 110(B12). 
Astarita, G. and Marrucci, G. (1974). Principles of non-Newtonian fluid mechanics, volume 28. McGraw-Hill New York.

Atzori, S., Tolomei, C., Antonioli, A., Merryman Boncori, J., Bannister, S., Trasatti, E., Pasquali, P., and Salvi, S. (2012). The 2010-2011 Canterbury, New Zealand, seismic sequence: multiple source analysis from InSAR data and modeling. Journal of Geophysical Research: Solid Earth, 117(B8).

Azúa, B. M., DeMets, C., and Masterlark, T. (2002). Strong interseismic coupling, fault afterslip, and viscoelastic flow before and after the Oct. 9, 1995 Colima-Jalisco earthquake: Continuous GPS measurements from Colima, Mexico. Geophysical research letters, 29(8):122-1.

Bamler, R. and Hartl, P. (1998). Synthetic aperture radar interferometry. Inverse problems, 14(4):R1.

Bannister, S., Fry, B., Reyners, M., Ristau, J., and Zhang, H. (2011). Fine-scale relocation of aftershocks of the 22 February $\mathrm{Mw}$ 6.2 Christchurch earthquake using double-difference tomography. Seismological Research Letters, 82(6):839-845.

Bannister, S. and Gledhill, K. (2012). Evolution of the 2010-2012 Canterbury earthquake sequence. New Zealand Journal of Geology and Geophysics, 55(3):295-304.

Barbot, S. and Fialko, Y. (2010a). A unified continuum representation of post-seismic relaxation mechanisms: semi-analytic models of afterslip, poroelastic rebound and viscoelastic flow. Geophysical Journal International, 182(3):1124-1140.

Barbot, S. and Fialko, Y. (2010b). A unified continuum representation of post-seismic relaxation mechanisms: Semi-analytic models of afterslip, poroelastic rebound and viscoelastic flow. Geophysical Journal International, 182(3):1124-1140. cited By (since 1996) 8.

Barbot, S., Fialko, Y., and Bock, Y. (2009). Postseismic deformation due to the Mw 6.02004 Parkfield earthquake: Stress-driven creep on a fault with spatially variable rate-and-state friction parameters. Journal of Geophysical Research: Solid Earth (1978-2012), 114(B7).

Barker, D. H., Sutherland, R., Henrys, S., and Bannister, S. (2009). Geometry of the Hikurangi subduction thrust and upper plate, North Island, New Zealand. Geochemistry, Geophysics, Geosystems, 10(2).

Barnes, P. M. and Lépinay, B. M. (1997). Rates and mechanics of rapid frontal accretion along the very obliquely convergent southern Hikurangi margin, New Zealand. Journal of Geophysical Research: Solid Earth (1978-2012), 102(B11):24931-24952. 
Barnes, P. M., Lépinay, B. M., Collot, J.-Y., Delteil, J., and Audru, J.-C. (1998). Strain partitioning in the transition area between oblique subduction and continental collision, Hikurangi margin, New Zealand. Tectonics, 17(4):534-557.

Barnes, P. M., Sutherland, R., and Delteil, J. (2005). Strike-slip structure and sedimentary basins of the southern Alpine Fault, Fiordland, New Zealand. Geological Society of America Bulletin, 117(3-4):411-435.

Barnhart, W. D., Willis, M. J., Lohman, R. B., and Melkonian, A. K. (2011). InSAR and optical constraints on fault slip during the 2010-2011 New Zealand earthquake sequence. Seismological Research Letters, 82(6):815-823.

Bartels, R. H. and Stewart, G. (1972). Solution of the matrix equation AX $+\mathrm{XB}=\mathrm{C}$ [F4]. Communications of the ACM, 15(9):820-826.

Batchelor, G. K. (2000). An introduction to fluid dynamics. Cambridge university press.

Beavan, J., Fielding, E., Motagh, M., Samsonov, S., and Donnelly, N. (2011). Fault location and slip distribution of the 22 February $2011 \mathrm{Mw} 6.2$ Christchurch, New Zealand, earthquake from geodetic data. Seismological Research Letters, 82(6):789799.

Beavan, J., Moore, M., Pearson, C., Henderson, M., Parsons, B., Bourne, S., England, P., Walcott, D., Blick, G., Darby, D., et al. (1999). Crustal deformation during 19941998 due to oblique continental collision in the central Southern Alps, New Zealand, and implications for seismic potential of the Alpine fault. Journal of Geophysical Research: Solid Earth (1978-2012), 104(B11):25233-25255.

Beavan, J., Motagh, M., Fielding, E., Donnelly, N., and Collett, D. (2012a). Fault slip models of the 2010-2011 Canterbury, New Zealand, earthquakes from geodetic data and observations of postseismic ground deformation. New Zealand Journal of Geology and Geophysics, 55(3):207-221.

Beavan, J., Motagh, M., Fielding, E. J., Donnelly, N., and Collett, D. (2012b). Fault slip models of the 2010-2011 Canterbury, New Zealand, earthquakes from geodetic data and observations of postseismic ground deformation. New Zealand Journal of Geology and Geophysics, 55(3):207-221.

Beavan, J., Samsonov, S., Denys, P., Sutherland, R., Palmer, N., and Denham, M. (2010a). Oblique slip on the Puysegur subduction interface in the 2009 July Mw 7.8 Dusky Sound earthquake from GPS and InSAR observations: implications for 
the tectonics of southwestern New Zealand. Geophysical Journal International, 183(3):1265-1286.

Beavan, J., Tregoning, P., Bevis, M., Kato, T., and Meertens, C. (2002). Motion and rigidity of the Pacific Plate and implications for plate boundary deformation. Journal of Geophysical Research: Solid Earth (1978-2012), 107(B10):ETG-19.

Beavan, J., Wallace, L., Samsonov, S., Ellis, S., Motagh, M., and Palmer, N. (2010b). The Darfield (Canterbury) earthquake: geodetic observations and preliminary source model. Bulletin of the New Zealand Society for Earthquake Engineering, 43(4):228.

Belytschko, T., Lu, Y. Y., Gu, L., et al. (1994). Element? free Galerkin methods. International journal for numerical methods in engineering, 37(2):229-256.

Benioff, H. (1951). Earthquakes and rock creep:(Part I: Creep characteristics of rocks and the origin of aftershocks). Bulletin of the Seismological Society of America, 41(1):31-62.

Berardino, P., Fornaro, G., Lanari, R., and Sansosti, E. (2002). A new algorithm for surface deformation monitoring based on small baseline differential SAR interferograms. Geoscience and Remote Sensing, IEEE Transactions on, 40(11):2375-2383.

Berryman, K., Beanland, S., Cooper, A., Cutten, H., Norris, R., and Wood, P. (1992). The Alpine Fault, New Zealand: variation in Quaternary structural style and geomorphic expression. In Annales tectonicae, volume 6, pages 126-163.

Bie, L., Ryder, I., Nippress, S. E., and Bürgmann, R. (2014). Coseismic and postseismic activity associated with the 2008 Mw 6.3 Damxung earthquake, Tibet, constrained by InSAR. Geophysical Journal International, 196(2):788-803.

Biggs, J., Burgmann, R., Freymueller, J. T., Lu, Z., Parsons, B., Ryder, I., Schmalzle, G., and Wright, T. (2009). The postseismic response to the 2002 M 7.9 Denali Fault earthquake: constraints from InSAR 2003-2005. Geophysical Journal International, $176(2): 353-367$.

Biot, M. A. (1941). General theory of three-dimensional consolidation. Journal of applied physics, 12(2):155-164.

Biot, M. A. (1962). Mechanics of deformation and acoustic propagation in porous media. Journal of applied physics, 33(4):1482-1498.

Bishop, D. (1985). Provisional terrane map of South Island, New Zealand. 
Booker, J. R. (1974). Time dependent strain following faulting of a porous medium. Journal of Geophysical Research, 79(14):2037-2044.

Borries, C., Jakowski, N., Jacobi, C., Hoffmann, P., and Pogoreltsev, A. (2007). Spectral analysis of planetary waves seen in ionospheric total electron content (TEC): First results using GPS differential TEC and stratospheric reanalyses. Journal of Atmospheric and Solar-Terrestrial Physics, 69(17):2442-2451.

Bosl, W. and Nur, A. (2002). Aftershocks and pore fluid diffusion following the 1992 Landers earthquake. Journal of Geophysical Research: Solid Earth (1978-2012), 107(B12):ESE-17.

Bradshaw, J. D. (1989). Cretaceous geotectonic patterns in the New Zealand region. Tectonics, 8(4):803-820.

Brenner, S. C. and Carstensen, C. (2004). Finite element methods. Encyclopedia of computational mechanics.

Bruhat, L., Barbot, S., and Avouac, J.-P. (2011). Evidence for postseismic deformation of the lower crust following the 2004 Mw6. 0 Parkfield earthquake. Journal of Geophysical Research: Solid Earth (1978-2012), 116(B8).

Bürgmann, R., Rosen, P. A., and Fielding, E. J. (2000). Synthetic aperture radar interferometry to measure Earth's surface topography and its deformation. Annual Review of Earth and Planetary Sciences, 28(1):169-209.

Calais, E., Vergnolle, M., Déverchère, J., San'kov, V., Lukhnev, A., and Amarjargal, S. (2002). Are post-seismic effects of the $\mathrm{M}=8.4$ Bolnay earthquake (1905 July 23) still influencing GPS velocities in the MongoliaBaikal area? Geophysical journal international, 149(1):157-168.

Carter, N. L. and Tsenn, M. C. (1987). Flow properties of continental lithosphere. Tectonophysics, 136(1):27-63.

Casarotti, E. and Piersanti, A. (2003). Postseismic stress diffusion in Chile and South Peru. Earth and Planetary Science Letters, 206(3):325-333.

Cattania, C., Hainzl, S., Wang, L., Enescu, B., and Roth, F. (2015). Aftershock triggering by postseismic stresses: A study based on Coulomb rate-and-state models. Journal of Geophysical Research: Solid Earth, 120(4):2388-2407.

Chan, C.-H. and Stein, R. S. (2009). Stress evolution following the 1999 Chi-Chi, Taiwan, earthquake: consequences for afterslip, relaxation, aftershocks and departures from Omori decay. Geophysical Journal International, 177(1):179-192. 
Chandrasekhar, D., Bürgmann, R., Reddy, C., Sunil, P., and Schmidt, D. A. (2009). Weak mantle in NW India probed by geodetic measurements following the 2001 Bhuj earthquake. Earth and Planetary Science Letters, 280(1):229-235.

Chang, W.-L., Smith, R. B., and Puskas, C. M. (2013). Effects of lithospheric viscoelastic relaxation on the contemporary deformation following the $1959 \mathrm{Mw} 7.3$ Hebgen Lake, Montana, earthquake and other areas of the intermountain seismic belt. Geochemistry, Geophysics, Geosystems, 14(1):1-17.

Chou, Y. (1966). Screw dislocations in and near lamellar inclusions. physica status solidi (b), 17(2):509-516.

Cocco, M. and Rice, J. R. (2002). Pore pressure and poroelasticity effects in Coulomb stress analysis of earthquake interactions. Journal of Geophysical Research: Solid Earth (1978-2012), 107(B2):ESE-2.

Coco, D. (1991). GPS-Satellites of opportunity for ionospheric monitoring. GPS world, $2(9): 47-50$.

Cooper, R. (1989). Early Paleozoic terranes of New Zealand. Journal of the Royal Society of New Zealand, 19(1):73-112.

Cooper, R. and Tulloch, A. (1992). Early Palaeozoic terranes in New Zealand and their relationship to the Lachlan Fold Belt. Tectonophysics, 214(1):129-144.

Cox, S., Rutter, H., Sims, A., Manga, M., Weir, J., Ezzy, T., White, P., Horton, T., and Scott, D. (2012). Hydrological effects of the MW 7.1 Darfield (Canterbury) earthquake, 4 September 2010, New Zealand. New Zealand Journal of Geology and Geophysics, 55(3):231-247.

Cox, S. C. and Sutherland, R. (2007). Regional geological framework of South Island, New Zealand, and its significance for understanding the active plate boundary. $A$ continental plate boundary: tectonics at South Island, New Zealand, pages 19-46.

Cubrinovski, M., Bray, J. D., Taylor, M., Giorgini, S., Bradley, B., Wotherspoon, L., and Zupan, J. (2011). Soil liquefaction effects in the central business district during the February 2011 Christchurch earthquake. Seismological Research Letters, 82(6):893-904.

Cubrinovski, M., Henderson, D., and Bradley, B. (2012). Liquefaction impacts in residential areas in the 2010-2011 Christchurch earthquakes. 
Cubrinovski, M. and Taylor, M. (2011). Liquefaction map of Christchurch based on drivethrough reconnaissance after the 22 February 2011 earthquake. University of Canterbury.

Dalla Via, G., De Natale, G., Troise, C., Pingue, F., Obrizzo, F., Riva, R., and Sabadini, R. (2003). First evidence of post-seismic deformation in the central Mediterranean: Crustal viscoelastic relaxation in the area of the 1980 Irpinia earthquake (Southern Italy). Geophysical Journal International, 154(3):F9-F14.

Dalla Via, G., Sabadini, R., De Natale, G., and Pingue, F. (2005). Lithospheric rheology in southern Italy inferred from postseismic viscoelastic relaxation following the 1980 Irpinia earthquake. Journal of Geophysical Research: Solid Earth (19782012), 110(B6).

Decriem, J. and Árnadóttir, T. (2012). Transient crustal deformation in the South Iceland Seismic Zone observed by GPS and InSAR during 2000-2008. Tectonophysics, 581:6-18.

Delteil, J., Collot, J.-Y., Wood, R., Herzer, R., Calmant, S., Christoffel, D., Coffin, M., Ferrière, J., Lamarche, G., Lebrun, J.-F., et al. (1996). From strike-slip faulting to oblique subduction: a survey of the Alpine Fault-Puysegur Trench transition, New Zealand, results of cruise Geodynz-sud leg 2. Marine geophysical researches, 18(2-4):383-399.

DeMets, C., Gordon, R. G., and Argus, D. F. (2010). Geologically current plate motions. Geophysical Journal International, 181(1):1-80.

Diao, F., Xiong, X., and Wang, R. (2011). Mechanisms of transient postseismic deformation following the $2001 \mathrm{Mw} 7.8$ Kunlun (China) earthquake. Pure and applied geophysics, 168(5):767-779.

Dieterich, J. (1994). A constitutive law for rate of earthquake production and its application to earthquake clustering. Journal of Geophysical Research: Solid Earth (1978-2012), 99(B2):2601-2618.

Dodson, A., Shardlow, P., Hubbard, L., Elgered, G., and Jarlemark, P. (1996). Wet tropospheric effects on precise relative GPS height determination. Journal of Geodesy, $70(4): 188-202$.

Douglas, A., Beavan, J., Wallace, L., and Townend, J. (2005). Slow slip on the northern Hikurangi subduction interface, New Zealand. Geophysical Research Letters, 32(16). 
Dzurisin, D. and Mori, Y. (2007). Volcano deformation: geodetic monitoring techniques. Springer Berlin.

Eberhart-Phillips, D. and Reyners, M. (1999). Plate interface properties in the northeast Hikurangi subduction zone, New Zealand, from converted seismic waves. Geophysical research letters, 26(16):2565-2568.

Eberhart-Phillips, D. and Reyners, M. (2001). A complex, young subduction zone imaged by three-dimensional seismic velocity, Fiordland, New Zealand. Geophysical Journal International, 146(3):731-746.

Eberhart-Phillips, D., Reyners, M., Bannister, S., Chadwick, M., and Ellis, S. (2010). Establishing a versatile 3-D seismic velocity model for New Zealand. Seismological Research Letters, 81(6):992-1000.

Eberhart-Phillips, D. and Stuart, W. D. (1992). Material heterogeneity simplifies the picture: Loma Prieta. Bulletin of the Seismological Society of America, 82(4):19641968.

Elachi, C. and Van Zyl, J. J. (2006). Introduction to the physics and techniques of remote sensing, volume 28. John Wiley \& Sons.

Elliott, J., Nissen, E., England, P., Jackson, J. A., Lamb, S., Li, Z., Oehlers, M., and Parsons, B. (2012). Slip in the 2010-2011 Canterbury earthquakes, New Zealand. Journal of Geophysical Research: Solid Earth (1978-2012), 117(B3).

Elsasser, W. M. (1967). Convection and stress propagation in the upper mantle.

Elsasser, W. M. (1969). Convection and stress propagation in the upper mantle.

Evans, E. L. and Meade, B. J. (2012). Geodetic imaging of coseismic slip and postseismic afterslip: Sparsity promoting methods applied to the great Tohoku earthquake. Geophysical Research Letters, 39(11).

Fernández, J. and Rundle, J. (2004). Postseismic viscoelastic-gravitational half space computations: Problems and solutions. Geophysical research letters, 31(7).

Fernández, J., Yu, T.-T., and Rundle, J. (1999). Relaxation models for earthquakes in layered viscoelastic Earth.

Fernández, J., Yu, T.-T., and Rundle, J. B. (1996). Deformation produced by a rectangular dipping fault in a viscoelastic-gravitational layered earth model. Part I: Thrust faultFLTGRV and FLTGRH FORTRAN programs. Computers \& Geosciences, 22(7):735-750. 
Ferretti, A., Monti-Guarnieri, A., Prati, C., Rocca, F., and Massonet, D. (2007). InSAR Principles-Guidelines for SAR Interferometry Processing and Interpretation, volume 19.

Ferretti, A., Prati, C., and Rocca, F. (2000). Nonlinear subsidence rate estimation using permanent scatterers in differential SAR interferometry. Geoscience and Remote Sensing, IEEE transactions on, 38(5):2202-2212.

Ferretti, A., Prati, C., and Rocca, F. (2001). Permanent scatterers in SAR interferometry. Geoscience and Remote Sensing, IEEE Transactions on, 39(1):8-20.

Fialko, Y., Sandwell, D., Simons, M., and Rosen, P. (2005). Three-dimensional deformation caused by the Bam, Iran, earthquake and the origin of shallow slip deficit. Nature, 435(7040):295-299.

Fick, A. (1855). Ueber diffusion. Annalen der Physik, 170(1):59-86.

Fielding, E. J., Lundgren, P. R., Bürgmann, R., and Funning, G. J. (2009). Shallow fault-zone dilatancy recovery after the 2003 Bam earthquake in Iran. Nature, 458(7234):64-68.

Findley, W., Lai, J., Onaran, K., and Christensen, R. (1977). Creep and Relaxation of Nonlinear Viscoelastic Materials With an Introduction to Linear Viscoelasticity. Journal of Applied Mechanics, 44:364.

Fliervoet, T. F., Drury, M. R., and Chopra, P. N. (1999). Crystallographic preferred orientations and misorientations in some olivine rocks deformed by diffusion or dislocation creep. Tectonophysics, 303(1):1-27.

Flügge, W. (1975). Viscoelasticity,(1975).

Forsythe, G. E. and Moler, C. B. (1967). Computer solution of linear algebraic systems, volume 7. Prentice-Hall Englewood Cliffs, NJ.

Freed, A. M. (2005). Earthquake triggering by static, dynamic, and postseismic stress transfer. Annu. Rev. Earth Planet. Sci., 33:335-367.

Freed, A. M. (2007). Afterslip (and only afterslip) following the 2004 Parkfield, California, earthquake. Geophysical Research Letters, 34(6).

Freed, A. M., Ali, S. T., and Bürgmann, R. (2007). Evolution of stress in Southern California for the past 200 years from coseismic, postseismic and interseismic stress changes. Geophysical Journal International, 169(3):1164-1179. 
Freed, A. M., Bürgmann, R., Calais, E., Freymueller, J., and Hreinsdóttir, S. (2006). Implications of deformation following the 2002 Denali, Alaska, earthquake for postseismic relaxation processes and lithospheric rheology. Journal of Geophysical Research: Solid Earth (1978-2012), 111(B1).

Freed, A. M., Hirth, G., and Behn, M. D. (2012). Using short-term postseismic displacements to infer the ambient deformation conditions of the upper mantle. Journal of Geophysical Research: Solid Earth (1978-2012), 117(B1).

Freed, A. M. and Lin, J. (1998). Time-dependent changes in failure stress following thrust earthquakes. Journal of Geophysical Research, 103(B10):24393-24409.

Freed, A. M. and Lin, J. (2001). Delayed triggering of the 1999 Hector Mine earthquake by viscoelastic stress transfer. Nature, 411(6834):180-183.

Fry, B., Bannister, S., Beavan, J., Bland, L., Bradley, B., Cox, S., Cousins, J., Gale, N., Hancox, G., Holden, C., et al. (2010). The MW 7.6 Dusky Sound earthquake of 2009: Preliminary report.

Fry, B., Benites, R., and Kaiser, A. (2011). The character of accelerations in the Mw 6.2 Christchurch earthquake. Seismological Research Letters, 82(6):846-852.

Fukahata, Y. and Matsu'ura, M. (2005). General expressions for internal deformation fields due to a dislocation source in a multilayered elastic half-space. Geophysical Journal International, 161(2):507-521.

Fukahata, Y. and Matsu'ura, M. (2006). Quasi-static internal deformation due to a dislocation source in a multilayered elastic/viscoelastic half-space and an equivalence theorem. Geophysical Journal International, 166(1):418-434.

Fukahata, Y., Nishitani, A., and Matsu'ura, M. (2004). Geodetic data inversion using ABIC to estimate slip history during one earthquake cycle with viscoelastic slipresponse functions. Geophysical Journal International, 156(1):140-153.

Fukuda, J., Higuchi, T., Miyazaki, S., and Kato, T. (2004). A new approach to timedependent inversion of geodetic data using a Monte Carlo mixture Kalman filter. Geophysical Journal International, 159(1):17-39.

Fukuda, J., Miyazaki, S., Higuchi, T., and Kato, T. (2008). Geodetic inversion for spacetime distribution of fault slip with time-varying smoothing regularization. Geophysical Journal International, 173(1):25-48.

Gilbert, K. E. (1983). A propagator matrix method for periodically stratified media. The Journal of the Acoustical Society of America, 73(1):137-142. 
Gledhill, K., Fry, B., Ristau, J., Holden, C., and Reyners, M. (2010). The darfield (canterbury) earthquake of september 2010: preliminary seismological report. Bulletin of the New Zealand Society for Earthquake Engineering, 43(4):215.

Gledhill, K., Ristau, J., Reyners, M., Fry, B., and Holden, C. (2011). The Darfield (Canterbury, New Zealand) Mw 7.1 earthquake of September 2010: A preliminary seismological report. Seismological Research Letters, 82(3):378-386.

Gualandi, A., Serpelloni, E., Elina Belardinelli, M., Bonafede, M., Pezzo, G., and Tolomei, C. (2015). Characterization of Ground Displacement Sources from Variational Bayesian Independent Component Analysis of Space Geodetic Time Series. In EGU General Assembly Conference Abstracts, volume 17, page 11093.

Gunawan, E., Sagiya, T., Ito, T., Kimata, F., Tabei, T., Ohta, Y., Meilano, I., Abidin, H. Z., Nurdin, I., Sugiyanto, D., et al. (2014). A comprehensive model of postseismic deformation of the 2004 Sumatra-Andaman earthquake deduced from GPS observations in northern Sumatra. Journal of Asian Earth Sciences, 88:218-229.

Hamling, I., D’Anastasio, E., Wallace, L., Ellis, S., Motagh, M., Samsonov, S., Palmer, N., and Hreinsdóttir, S. (2014). Crustal deformation and stress transfer during a propagating earthquake sequence: The 2013 Cook Strait sequence, central New Zealand. Journal of Geophysical Research: Solid Earth, 119(7):6080-6092.

Hammond, W. C., Kreemer, C., and Blewitt, G. (2009). Geodetic constraints on contemporary deformation in the northern Walker Lane: 3. Central Nevada seismic belt postseismic relaxation. Geological Society of America Special Papers, 447:33-54.

Han, S.-C., Sauber, J., Luthcke, S. B., Ji, C., and Pollitz, F. F. (2008). Implications of postseismic gravity change following the great 2004 Sumatra-Andaman earthquake from the regional harmonic analysis of GRACE intersatellite tracking data. Journal of Geophysical Research: Solid Earth (1978-2012), 113(B11).

Hao, M., Shen, Z.-K., Wang, Q., and Cui, D. (2012). Postseismic deformation mechanisms of the $1990 \mathrm{Mw}$ 6.4 Gonghe, China earthquake constrained using leveling measurements. Tectonophysics, 532:205-214.

Harris, R. A. (1998). Introduction to special section: Stress triggers, stress shadows, and implications. J. geophys. Res, 103(24):347-24.

Haskell, N. A. (1953). The dispersion of surface waves on multilayered media. Bulletin of the seismological Society of America, 43(1):17-34. 
He, J., Xia, W., Lu, S., and Qian, H. (2011). Three-dimensional finite element modeling of stress evolution around the Xiaojiang fault system in the southeastern Tibetan plateau during the past ${ }^{\sim}$ 500years. Tectonophysics, 507(1):70-85.

Hearn, E., McClusky, S., Ergintav, S., and Reilinger, R. (2009). Izmit earthquake postseismic deformation and dynamics of the North Anatolian Fault Zone. Journal of Geophysical Research: Solid Earth (1978-2012), 114(B8).

Hearn, E. H., Bürgmann, R., and Reilinger, R. E. (2002a). Dynamics of Izmit earthquake postseismic deformation and loading of the Düzce earthquake hypocenter. Bulletin of the Seismological Society of America, 92(1):172-193.

Hearn, E. H., Hager, B. H., and Reilinger, R. E. (2002b). Viscoelastic deformation from North Anatolian Fault Zone earthquakes and the eastern Mediterranean GPS velocity field. Geophysical research letters, 29(11):44-1.

Heflin, M., Bertiger, W., Blewitt, G., Freedman, A., Hurst, K., Lichten, S., Lindqwister, U., Vigue, Y., Webb, F., Yunck, T., et al. (1992). Global geodesy using GPS without fiducial sites. Geophysical Research Letters, 19(2):131-134.

Hergert, T. and Heidbach, O. (2006). New insights into the mechanism of postseismic stress relaxation exemplified by the 23 June $2001 \mathrm{Mw}=8.4$ earthquake in southern Peru. Geophysical research letters, 33(2).

Herman, M. W., Herrmann, R. B., Benz, H. M., and Furlong, K. P. (2014). Using regional moment tensors to constrain the kinematics and stress evolution of the 20102013 Canterbury earthquake sequence, South Island, New Zealand. Tectonophysics, 633:1-15.

Herring, T. (2003). Results and Comparisions from the Southern California Integrated and other arrays. In AGU Fall Meeting Abstracts, volume 1, page 04.

Herring, T., King, R., and McClusky, S. (2008). Introduction to Gamit/Globk. Massachusetts Institute of Technology, Cambridge.

Hetland, E. and Hager, B. (2003). Postseismic relaxation across the central Nevada seismic belt. Journal of Geophysical Research: Solid Earth (1978-2012), 108(B8).

Hetland, E. and Hager, B. (2005). Postseismic and interseismic displacements near a strike-slip fault: A two-dimensional theory for general linear viscoelastic rheologies. Journal of Geophysical Research: Solid Earth (1978-2012), 110(B10). 
Hoechner, A., Sobolev, S. V., Einarsson, I., and Wang, R. (2011). Investigation on afterslip and steady state and transient rheology based on postseismic deformation and geoid change caused by the Sumatra 2004 earthquake. Geochemistry, Geophysics, Geosystems, 12(7).

Holdahl, S. R. and Dzurisin, D. (1991). Time-dependent models of vertical deformation for the Yellowstone-Hebgen Lake Region, 1923-1987. Journal of Geophysical Research: Solid Earth (1978-2012), 96(B2):2465-2483.

Holden, C. (2011). Kinematic source model of the 22 February 2011 Mw 6.2 Christchurch earthquake using strong motion data. Seismological research letters, $82(6): 783-788$.

Holden, C., Beavan, J., Fry, B., Reyners, M., Ristau, J., Van Dissen, R., Villamor, P., and Quigley, M. (2011). Preliminary source model of the Mw 7.1 Darfield earthquake from geological, geodetic and seismic data. In Proceedings of the Ninth Pacific Conference on Earthquake Engineering, Building an Earthquake-Resilient Society, pages $14-16$.

Holden, C., Kaiser, A., Van Dissen, R., and Jury, R. (2013). Sources, ground motion and structural response characteristics in Wellington of the 2013 Cook Strait earthquakes. Bull. New Zeal. Soc. Earthq. Eng., 46(4):188-195.

Holt, R., Savage, M., Townend, J., Syracuse, E., and Thurber, C. (2013). Crustal stress and fault strength in the Canterbury Plains, New Zealand. Earth and Planetary Science Letters, 383:173-181.

Holt, W. E. and Haines, A. (1995). The kinematics of northern South Island, New Zealand, determined from geologic strain rates. Journal of Geophysical Research: Solid Earth (1978-2012), 100(B9):17991-18010.

Hooper, A. (2008). A multi-temporal InSAR method incorporating both persistent scatterer and small baseline approaches. Geophysical Research Letters, 35(16).

Hooper, A., Spaans, K., Bekaert, D., Cuenca, M. C., Arıkan, M., and Oyen, A. (2010). StaMPS/MTI Manual. Delft Institute of Earth Observation and Space Systems Delft University of Technology, Kluyverweg, 1:2629.

Hooper, A., Zebker, H., Segall, P., and Kampes, B. (2004). A new method for measuring deformation on volcanoes and other natural terrains using InSAR persistent scatterers. Geophysical Research Letters, 31(23):1-5. 
Hooper, A. and Zebker, H. A. (2007). Phase unwrapping in three dimensions with application to InSAR time series. JOSA A, 24(9):2737-2747.

Hooper, A. J. (2006). Persistent scatter radar interferometry for crustal deformation studies and modeling of volcanic deformation.

Hsu, Y.-J., Bechor, N., Segall, P., Yu, S.-B., Kuo, L.-C., and Ma, K.-F. (2002). Rapid afterslip following the 1999 Chi-Chi, Taiwan earthquake. Geophysical Research Letters, 29(16).

Hsu, Y.-J., Simons, M., Avouac, J.-P., Galetzka, J., Sieh, K., Chlieh, M., Natawidjaja, D., Prawirodirdjo, L., and Bock, Y. (2006). Frictional afterslip following the 2005 Nias-Simeulue earthquake, Sumatra. Science, 312(5782):1921-1926.

Hu, Y., Bürgmann, R., Freymueller, J. T., Banerjee, P., and Wang, K. (2014). Contributions of poroelastic rebound and a weak volcanic arc to the postseismic deformation of the 2011 Tohoku earthquake. Earth, Planets and Space, 66(1):1-10.

Hu, Y., Wang, K., He, J., Klotz, J., and Khazaradze, G. (2004). Three-dimensional viscoelastic finite element model for postseismic deformation of the great 1960 Chile earthquake. Journal of Geophysical Research: Solid Earth (1978-2012), 109(B12).

Hughes, K. L., Masterlark, T., and Mooney, W. D. (2010). Poroelastic stress-triggering of the 2005 M8. 7 Nias earthquake by the 2004 M9. 2 Sumatra-Andaman earthquake. Earth and Planetary Science Letters, 293(3):289-299.

Hughes, K. L., Masterlark, T., and Mooney, W. D. (2011). Pore-fluid migration and the timing of the 2005 M8. 7 Nias earthquake. Lithosphere, 3(2):170-172.

Hughes, T. J. (2012). The finite element method: linear static and dynamic finite element analysis. Courier Corporation.

Ide, S., Takeo, M., and Yoshida, Y. (1996). Source process of the 1995 Kobe earthquake: Determination of spatio-temporal slip distribution by Bayesian modeling. Bulletin of the Seismological society of America, 86(3):547-566.

Ivins, E. R. (1996). Transient creep of a composite lower crust: 2. A polymineralic basis for rapidly evolving postseismic deformation modes. Journal of Geophysical Research: Solid Earth (1978-2012), 101(B12):28005-28028.

Iwasaki, T. (1985). Quasi-static deformation due to a dislocation source in a Maxwellian viscoelastic earth model. Journal of Physics of the Earth, 33(1):21-43.

Jeffreys, S. H. (1961). Theory of probability. 
Johnson, K., Fukuda, J., and Segall, P. (2012). Challenging the rate-state asperity model: Afterslip following the 2011 Mw9 Tohoku-oki, Japan, earthquake. Geophysical Research Letters, 39(20). cited By (since 1996) 0.

Johnson, K. and Segall, P. (2004). Viscoelastic earthquake cycle models with deep stress-driven creep along the San Andreas fault system. Journal of Geophysical Research: Solid Earth (1978-2012), 109(B10).

Johnson, K. M., Bürgmann, R., and Freymueller, J. T. (2009). Coupled afterslip and viscoelastic flow following the 2002 Denali Fault, Alaska earthquake. Geophysical Journal International, 176(3):670-682.

Johnson, K. M., Bürgmann, R., and Larson, K. (2006). Frictional properties on the San Andreas fault near Parkfield, California, inferred from models of afterslip following the 2004 earthquake. Bulletin of the Seismological Society of America, 96(4B):S321S338.

Johnson, K. M., Hilley, G. E., and Bürgmann, R. (2007). Influence of lithosphere viscosity structure on estimates of fault slip rate in the Mojave region of the San Andreas fault system. Journal of Geophysical Research: Solid Earth (1978-2012), $112(\mathrm{~B} 7)$.

Jónsson, S. (2008). Importance of post-seismic viscous relaxation in southern Iceland. Nature Geoscience, 1(2):136-139.

Jonsson, S., Segall, P., Pedersen, R., and Björnsson, G. (2003). Post-earthquake ground movements correlated to pore-pressure transients. Nature, 424(6945):179-183.

Kaiser, A., Holden, C., Beavan, J., Beetham, D., Benites, R., Celentano, A., Collett, D., Cousins, J., Cubrinovski, M., Dellow, G., et al. (2012). The Mw 6.2 Christchurch earthquake of February 2011: preliminary report. New Zealand journal of geology and geophysics, 55(1):67-90.

Kalman, R. E. (1960). A new approach to linear filtering and prediction problems. Journal of Fluids Engineering, 82(1):35-45.

Kampes, B. and Usai, S. (1999). Doris: The delft object-oriented radar interferometric software. In 2nd international symposium on operationalization of remote sensing, enschede, the netherlands, volume 16, page 20. Citeseer.

Kampes, B. M. (2005). Displacement parameter estimation using permanent scatterer interferometry. PhD thesis, TU Delft, Delft University of Technology. 
Karato, S.-I., Paterson, M. S., and FitzGerald, J. D. (1986). Rheology of synthetic olivine aggregates: influence of grain size and water. Journal of Geophysical Research: Solid Earth (1978-2012), 91(B8):8151-8176.

Karato, S.-I. and Wu, P. (1993). Rheology of the upper mantle- A synthesis. Science, 260(5109):771-778.

Kawamura, M. and Yamaoka, K. (2006). Spatiotemporal characteristics of the displacement field revealed with principal component analysis and the mode-rotation technique. Tectonophysics, 419(1):55-73.

Kawamura, M. and Yamaoka, K. (2009). Temporal relationship between the 2000 Miyake-Kozu seismovolcanic activity and the 2000 Tokai slow-slip event. Tectonophysics, 465(1):45-59.

Kenner, S. and Segall, P. (1999). Time-dependence of the stress shadowing effect and its relation to the structure of the lower crust. Geology, 27(2):119-122.

Khazaradze, G., Wang, K., Klotz, J., Hu, Y., and He, J. (2002). Prolonged postseismic deformation of the 1960 great Chile earthquake and implications for mantle rheology. Geophysical Research Letters, 29(22):7-1.

King, N., Svarc, J., Fogleman, E., Gross, W., Clark, K., Hamilton, G., Stiffler, C., and Sutton, J. (1995). Continuous GPS observations across the Hayward fault, California, 1991-1994. Journal of Geophysical Research: Solid Earth (1978-2012), 100(B10):20271-20283.

Kirby, B. J. (2010). Micro-and nanoscale fluid mechanics: transport in microfluidic devices. Cambridge University Press.

Kirby, S. and Kronenberg, A. (1987). Rheology of the lithosphere: selected topics. Reviews of Geophysics, 25(6):1219-1244.

Kitagawa, G. and Gersch, W. (2012). Smoothness priors analysis of time series, volume 116. Springer Science \& Business Media.

Kogan, M. G., Vasilenko, N. F., Frolov, D. I., Freymueller, J. T., Steblov, G. M., Levin, B. W., and Prytkov, A. S. (2011). The mechanism of postseismic deformation triggered by the 2006-2007 great Kuril earthquakes. Geophysical Research Letters, $38(6)$.

Kogan, M. G., Vasilenko, N. F., Frolov, D. I., Freymueller, J. T., Steblov, G. M., Prytkov, A. S., and Ekström, G. (2013). Rapid postseismic relaxation after the 
great 2006-2007 Kuril earthquakes from GPS observations in 2007-2011. Journal of Geophysical Research: Solid Earth, 118(7):3691-3706.

Koseluk, R. A. and Bischke, R. E. (1981). An elastic rebound model for normal fault earthquakes. Journal of Geophysical Research: Solid Earth (1978-2012), 86(B2):1081-1090.

Kositsky, A. and Avouac, J.-P. (2010). Inverting geodetic time series with a principal component analysis-based inversion method. Journal of Geophysical Research: Solid Earth (1978-2012), 115(B3).

Kozuch, M. J., Yu, T.-T., and Rundle, J. B. (1996). Southeastern Caribbean sea level variability and viscoelastic relaxation. Journal of Geophysical Research: Solid Earth (1978-2012), 101(B4):8579-8593.

Kundu, P. and Cohen, L. (1990). Fluid Mechanics, 638 pp. Academic, Calif.

Lanari, R., Casu, F., Manzo, M., Zeni, G., Berardino, P., Manunta, M., and Pepe, A. (2007). An overview of the small baseline subset algorithm: A DInSAR technique for surface deformation analysis. Pure and Applied Geophysics, 164(4):637-661.

Lapusta, N., Rice, J. R., Ben-Zion, Y., and Zheng, G. (2000). Elastodynamic analysis for slow tectonic loading with spontaneous rupture episodes on faults with rate-and state-dependent friction. Journal of Geophysical Research: Solid Earth (1978-2012), 105(B10):23765-23789.

Le Pourhiet, L., Huet, B., and Traoré, N. (2014). Links between long-term and shortterm rheology of the lithosphere: Insights from strike-slip fault modelling. Tectonophysics, 631:146-159.

Lebrun, J.-F., Lamarche, G., Collot, J.-Y., and Delteil, J. (2000). Abrupt strike-slip fault to subduction transition: the Alpine Fault-Puysegur Trench connection, New Zealand. Tectonics, 19(4):688-706.

Li, Z., Qu, W., Young, K., and Zhang, Q. (2011). Earthquake source parameters of the 2009M W7. 8 Fiordland (New Zealand) earthquake from L-band InSAR observations. Earthquake Science, 24(2):199-206.

Linker, M. and Rice, J. (1997). Models of postseismic deformation and stress transfer associated with the Loma Prieta earthquake. US Geological Survey professional paper, (1550D).

Lomnitz, C. (1956). Creep measurements in igneous rocks. The Journal of Geology, pages $473-479$. 
Lorenzo-Martín, F., Roth, F., and Wang, R. (2006). Inversion for rheological parameters from post-seismic surface deformation associated with the 1960 Valdivia earthquake, Chile. Geophysical Journal International, 164(1):75-87.

Lu, Y., Belytschko, T., and Gu, L. (1994). A new implementation of the element free Galerkin method. Computer methods in applied mechanics and engineering, 113(3):397-414.

Lubis, A., Hashima, A., and Sato, T. (2013). Analysis of afterslip distribution following the 2007 September 12 southern Sumatra earthquake using poroelastic and viscoelastic media. Geophysical Journal International, 192(1):18-37.

Lubis, A. M., Hashima, A., and Sato, T. (2012). Analysis of afterslip distribution following the 2007 September 12 southern Sumatra earthquake using poroelastic and viscoelastic media. Geophysical Journal International, page ggs020.

Lyard, F., Lefevre, F., Letellier, T., and Francis, O. (2006). Modelling the global ocean tides: modern insights from FES2004. Ocean Dynamics, 56(5-6):394-415.

MacKay, D. J. (1992). Bayesian interpolation. Neural computation, 4(3):415-447.

MacKinnon, T. C. (1983). Origin of the Torlesse terrane and coeval rocks, South Island, New Zealand. Geological Society of America Bulletin, 94(8):967-985.

Malservisi, R., Furlong, K. P., and Anderson, H. (2003). Dynamic uplift in a transpressional regime: numerical model of the subduction area of Fiordland, New Zealand. Earth and Planetary Science Letters, 206(3):349-364.

Malys, S., Larezos, M., Gottschalk, S., Mobbs, S., Winn, B., Feess, W., Menn, M., Swift, E., Merrigan, M., and Mathon, W. (1997). The GPS accuracy improvement initiative. In Proceedings of the 10th International Technical Meeting of the Satellite Division of The Institute of Navigation (ION GPS 1997), pages 375-384.

Marone, C. J., Scholtz, C., and Bilham, R. (1991). On the mechanics of earthquake afterslip. Journal of Geophysical Research: Solid Earth (1978-2012), 96(B5):84418452 .

Massonnet, D., Rossi, M., Carmona, C., Adragna, F., Peltzer, G., Feigl, K., and Rabaute, T. (1993). The displacement field of the Landers earthquake mapped by radar interferometry. Nature, 364(6433):138-142.

Masterlark, T. (2003). Finite element model predictions of static deformation from dislocation sources in a subduction zone: Sensitivities to homogeneous, isotropic, 
Poisson-solid, and half-space assumptions. Journal of Geophysical Research: Solid Earth (1978-2012), 108(B11).

Masterlark, T., DeMets, C., Wang, H., Sanchez, O., and Stock, J. (2001). Homogeneous vs heterogeneous subduction zone models: Coseismic and postseismic deformation. Geophysical Research Letters, 28(21):4047-4050.

Masterlark, T. and Wang, H. F. (2002). Transient stress-coupling between the 1992 Landers and 1999 Hector Mine, California, earthquakes. Bulletin of the Seismological Society of America, 92(4):1470-1486.

Matsu'ura, M. and Iwasaki, T. (1983). Study on coseismic and postseismic crustal movements associated with the 1923 Kanto earthquake. Tectonophysics, 97(1):201215.

McCaffrey, R., Wallace, L. M., and Beavan, J. (2008). Slow slip and frictional transition at low temperature at the Hikurangi subduction zone. Nature Geoscience, 1(5):316320.

McGinty, P. and Robinson, R. (2007). The 2003 Mw 7.2 Fiordland subduction earthquake, New Zealand: aftershock distribution, main shock fault plane and static stress changes on the overlying Alpine Fault. Geophysical Journal International, $169(2): 579-592$.

Miller, D. (1988). The iterative solution of the matrix equation $\mathrm{XA}+\mathrm{BX}+\mathrm{C}=0$. Linear Algebra and Its Applications, 105:131-137.

Misra, P. and Enge, P. (2006). Global Positioning System: Signals, Measurements and Performance Second Edition. Lincoln, MA: Ganga-Jamuna Press.

Mitrovica, J. and Peltier, W. (1992). A comparison of methods for the inversion of viscoelastic relaxation spectra. Geophysical journal international, 108(2):410-414.

Miyazaki, S.-i., Segall, P., Fukuda, J., and Kato, T. (2004). Space time distribution of afterslip following the 2003 Tokachi-oki earthquake: Implications for variations in fault zone frictional properties. Geophysical Research Letters, 31(6).

Mogi, K. (1958). Relations between the eruptions of various volcanoes and the deformations of the ground surfaces around them.

Montési, L. G. (2004). Controls of shear zone rheology and tectonic loading on postseismic creep. Journal of Geophysical Research: Solid Earth (1978-2012), 109(B10). 
Montési, L. G. and Hirth, G. (2003). Grain size evolution and the rheology of ductile shear zones: from laboratory experiments to postseismic creep. Earth and Planetary Science Letters, 211(1):97-110.

Mortimer, N. (2004). New Zealand's geological foundations. Gondwana Research, $7(1): 261-272$.

Motagh, M., Beavan, J., Fielding, E. J., and Haghshenas, M. (2014a). Postseismic Ground Deformation Following the September 2010 Darfield, New Zealand, Earthquake From TerraSAR-X, COSMO-SkyMed, and ALOS InSAR. Geoscience and Remote Sensing Letters, IEEE, 11(1):186-190.

Motagh, M., Beavan, J., Fielding, E. J., and Haghshenas, M. (2014b). Postseismic Ground Deformation Following the September 2010 Darfield, New Zealand, Earthquake From TerraSAR-X, COSMO-SkyMed, and ALOS InSAR. Geoscience and Remote Sensing Letters, IEEE, 11(1):186-190.

Mucciarelli, M. (2011). Ambient noise measurements following the 2011 Christchurch earthquake: relationships with previous microzonation studies, liquefaction, and nonlinearity. Seismological Research Letters, 82(6):919-926.

Murray-Moraleda, J. R. and Simpson, R. W. (2009). Geodetically inferred coseismic and postseismic slip due to the M 5.431 October 2007 Alum Rock earthquake. Bulletin of the Seismological Society of America, 99(5):2784-2800.

Muto, J., Shibazaki, B., Ito, Y., Inuma, T., Ohzono, M., Matsumoto, T., and Okada, T. (2013). Two-dimensional viscosity structure of the northeastern Japan islands arc-trench system. Geophysical Research Letters, 40(17):4604-4608.

Nathan, S. (1976). Geochemistry of the Greenland Group (Early Ordovician), New Zealand. New Zealand journal of geology and geophysics, 19(5):683-706.

Nayroles, B., Touzot, G., and Villon, P. (1992). Generalizing the finite element method: diffuse approximation and diffuse elements. Computational mechanics, 10(5):307318.

Nishimura, T. and Thatcher, W. (2003). Rheology of the lithosphere inferred from postseismic uplift following the 1959 Hebgen Lake earthquake. Journal of Geophysical Research: Solid Earth (1978-2012), 108(B8).

Norris, R., Koons, P., and Cooper, A. (1990). The obliquely-convergent plate boundary in the South Island of New Zealand: implications for ancient collision zones. Journal of structural geology, 12(5):715-725. 
Norris, R. J. and Cooper, A. F. (2001). Late Quaternary slip rates and slip partitioning on the Alpine Fault, New Zealand. Journal of Structural Geology, 23(2):507-520.

Nur, A. (1971). Effects of stress on velocity anisotropy in rocks with cracks. Journal of Geophysical Research, 76(8):2022-2034.

Nur, A. and Byerlee, J. (1971). An exact effective stress law for elastic deformation of rock with fluids. Journal of Geophysical Research, 76(26):6414-6419.

Nur, A. and Mavko, G. (1974). Postseismic viscoelastic rebound. Science, 183(4121):204-206.

Ohzono, M., Ohta, Y., Iinuma, T., Miura, S., and Muto, J. (2012). Geodetic evidence of viscoelastic relaxation after the 2008 Iwate-Miyagi Nairiku earthquake. Earth, planets and space, 64(9):759-764.

Okada, Y. (1985). Surface deformation due to shear and tensile faults in a half-space. Bulletin of the seismological society of America, 75(4):1135-1154.

Palano, M., Gresta, S., and Puglisi, G. (2009). Time-dependent deformation of the eastern flank of Mt. Etna: After-slip or viscoelastic relaxation? Tectonophysics, $473(3): 300-311$.

Panet, I., Pollitz, F., Mikhailov, V., Diament, M., Banerjee, P., and Grijalva, K. (2010). Upper mantle rheology from GRACE and GPS postseismic deformation after the 2004 Sumatra-Andaman earthquake. Geochemistry, Geophysics, Geosystems, 11(6).

Parkinson, B. W. and Enge, P. K. (1996). Differential gps. Global Positioning System: Theory and applications., 2:3-50.

Paterson, M. (1987). Problems in the extrapolation of laboratory rheological data. Tectonophysics, 133(1):33-43.

Paul, J., Lowry, A., Bilham, R., Sen, S., and Smalley, R. (2007). Postseismic deformation of the Andaman Islands following the 26 December, 2004 Great SumatraAndaman earthquake. Geophysical Research Letters, 34(19).

Peltier, W. (1985). The LAGEOS constraint on deep mantle viscosity: results from a new normal mode method for the inversion of viscoelastic relaxation spectra. Journal of Geophysical Research: Solid Earth (1978-2012), 90(B11):9411-9421.

Peltzer, G., Rosen, P., Rogez, F., and Hudnut, K. (1996). Postseismic rebound in fault step-overs caused by pore fluid flow. SCIENCE-NEW YORK THEN WASHINGTON-, pages 1202-1203. 
Peltzer, G., Rosen, P., Rogez, F., and Hudnut, K. (1998). Poroelastic rebound along the Landers 1992 earthquake surface rupture. Journal of Geophysical Research: Solid Earth (1978-2012), 103(B12):30131-30145.

Peng, Z. and Ben-Zion, Y. (2006). Temporal changes of shallow seismic velocity around the Karadere-Düzce branch of the north Anatolian fault and strong ground motion. Pure and Applied Geophysics, 163(2-3):567-600.

Perfettini, H. and Ampuero, J.-P. (2008). Dynamics of a velocity strengthening fault region: Implications for slow earthquakes and postseismic slip. Journal of Geophysical Research: Solid Earth (1978-2012), 113(B9).

Perfettini, H. and Avouac, J. (2007). Modeling afterslip and aftershocks following the 1992 Landers earthquake-art. no. B07409. Journal of Geophysical Research Solid Earth, 112(B7):NIL_1-NIL_19.

Perfettini, H. and Avouac, J.-P. (2004). Postseismic relaxation driven by brittle creep: A possible mechanism to reconcile geodetic measurements and the decay rate of aftershocks, application to the Chi-Chi earthquake, Taiwan. Journal of Geophysical Research: Solid Earth (1978-2012), 109(B2).

Perfettini, H., Avouac, J.-P., and Ruegg, J.-C. (2005). Geodetic displacements and aftershocks following the $2001 \mathrm{Mw}=8.4$ Peru earthquake: Implications for the mechanics of the earthquake cycle along subduction zones. Journal of Geophysical Research: Solid Earth (1978-2012), 110(B9).

Piersanti, A. (1999). Postseismic deformation in Chile: Constraints on the asthenospheric viscosity. Geophysical Research Letters, 26(20):3157-3160.

Piersanti, A., Nostro, C., and Riguzzi, F. (2001). Active displacement field in the Suez-Sinai area: the role of postseismic deformation. Earth and Planetary Science Letters, 193(1):13-23.

Piombo, A., Martinelli, G., and Dragoni, M. (2005). Post-seismic fluid flow and Coulomb stress changes in a poroelastic medium. Geophysical Journal International, 162(2):507-515.

Podgorski, J., Hearn, E., McClusky, S., Reilinger, R., Taymaz, T., Tan, O., Prilepin, M., Guseva, T., and Nadariya, M. (2007). Postseismic deformation following the 1991 Racha, Georgia, earthquake. Geophysical research letters, 34(4).

Pollitz, F., Banerjee, P., Grijalva, K., Nagarajan, B., and Bürgmann, R. (2008). Effect of $3-\mathrm{D}$ viscoelastic structure on post-seismic relaxation from the $2004 \mathrm{M}=9.2$ Sumatra earthquake. Geophysical Journal International, 173(1):189-204. 
Pollitz, F. F. (1992). Postseismic relaxation theory on the spherical earth. Bulletin of the Seismological Society of America, 82(1):422-453.

Pollitz, F. F. (1997). Gravitational viscoelastic postseismic relaxation on a layered spherical Earth. Journal of Geophysical Research: Solid Earth (1978-2012), 102(B8):17921-17941.

Pollitz, F. F. (2003a). Post-seismic relaxation theory on a laterally heterogeneous viscoelastic model. Geophysical Journal International, 155(1):57-78.

Pollitz, F. F. (2003b). Transient rheology of the uppermost mantle beneath the Mojave Desert, California. Earth and Planetary Science Letters, 215(1):89-104.

Pollitz, F. F. (2005). Transient rheology of the upper mantle beneath central Alaska inferred from the crustal velocity field following the 2002 Denali earthquake. Journal of Geophysical Research: Solid Earth (1978-2012), 110(B8).

Pollitz, F. F. (2015). Postearthquake relaxation evidence for laterally variable viscoelastic structure and water content in the Southern California mantle. Journal of Geophysical Research: Solid Earth.

Pollitz, F. F., Banerjee, P., Bürgmann, R., Hashimoto, M., and Choosakul, N. (2006a). Stress changes along the Sunda trench following the 26 December 2004 SumatraAndaman and 28 March 2005 Nias earthquakes. Geophysical research letters, 33(6).

Pollitz, F. F., Bürgmann, R., and Banerjee, P. (2006b). Post-seismic relaxation following the great 2004 Sumatra-Andaman earthquake on a compressible self-gravitating Earth. Geophysical Journal International, 167(1):397-420.

Pollitz, F. F., Bürgmann, R., and Romanowicz, B. (1998a). Viscosity of oceanic asthenosphere inferred from remote triggering of earthquakes. Science, 280(5367):12451249 .

Pollitz, F. F., Bürgmann, R., and Segall, P. (1998b). Joint estimation of afterslip rate and postseismic relaxation following the 1989 Loma Prieta earthquake. Journal of Geophysical Research: Solid Earth (1978-2012), 103(B11):26975-26992.

Pollitz, F. F., Bürgmann, R., and Thatcher, W. (2012). Illumination of rheological mantle heterogeneity by the M7. 22010 El Mayor-Cucapah earthquake. Geochemistry, Geophysics, Geosystems, 13(6).

Pollitz, F. F. and Dixon, T. H. (1998). GPS measurements across the northern Caribbean plate boundary zone: Impact of postseismic relaxation following historic earthquakes. Geophysical research letters, 25(12):2233-2236. 
Pollitz, F. F., Nyst, M., Nishimura, T., and Thatcher, W. (2006c). Inference of postseismic deformation mechanisms of the 1923 Kanto earthquake. Journal of Geophysical Research: Solid Earth (1978-2012), 111(B5).

Pollitz, F. F., Peltzer, G., and Bürgmann, R. (2000). Mobility of continental mantle: Evidence from postseismic geodetic observations following the 1992 Landers earthquake. Journal of Geophysical Research: Solid Earth (1978-2012), 105(B4):80358054 .

Pollitz, F. F. and Sacks, I. S. (1994). Fault Model of the 1891 Nobi Earthquake from Historic Triangulation and Leveling. Journal of Physics of the Earth, 42(1):1-43.

Pollitz, F. F. and Sacks, I. S. (1996). Viscosity structure beneath northeast Iceland. Journal of Geophysical Research: Solid Earth (1978-2012), 101(B8):17771-17793.

Pollitz, F. F. and Sacks, I. S. (2002). Stress triggering of the 1999 Hector Mine earthquake by transient deformation following the 1992 Landers earthquake. Bulletin of the Seismological Society of America, 92(4):1487-1496.

Pritchard, M. and Simons, M. (2006). An aseismic slip pulse in northern Chile and along-strike variations in seismogenic behavior. Journal of Geophysical Research: Solid Earth (1978-2012), 111(B8).

Quigley, M., Van Dissen, R., Litchfield, N., Villamor, P., Duffy, B., Barrell, D., Furlong, K., Stahl, T., Bilderback, E., and Noble, D. (2012). Surface rupture during the 2010 Mw 7.1 Darfield (Canterbury) earthquake: Implications for fault rupture dynamics and seismic-hazard analysis. Geology, 40(1):55-58.

Quigley, M. C., Bastin, S., and Bradley, B. A. (2013). Recurrent liquefaction in Christchurch, New Zealand, during the Canterbury earthquake sequence. Geology, 41(4):419-422.

Reddy, C., Prajapati, S., Sunil, P., and Arora, S. (2012). Transient postseismic mantle relaxation following 2004 Sumatra earthquake: implications of seismic vulnerability in the Andaman-Nicobar region. Natural Hazards and Earth System Science, $12(2): 431-441$.

Reddy, C., Prajapati, S. K., and Sunil, P. (2010). Co and postseismic characteristics of Indian sub-continent in response to the 2004 Sumatra earthquake. Journal of Asian Earth Sciences, 39(6):620-626.

Reddy, C., Sunil, P., Bürgmann, R., Chandrasekhar, D., and Kato, T. (2013). Postseismic relaxation due to Bhuj earthquake on January 26, 2001: possible mechanisms and processes. Natural hazards, 65(2):1119-1134. 
Reddy, J. N. (1989). An introduction to the finite element method.

Reddy, J. N. (1993). An introduction to the finite element method, volume 2. McGrawHill New York.

Reilinger, R. (1986). Evidence for postseismic viscoelastic relaxation following the 1959 $\mathrm{M}=7.5$ Hebgen Lake, Montana, earthquake. Journal of Geophysical Research: Solid Earth (1978-2012), 91(B9):9488-9494.

Remondi, B. (1988). Kinematic and pseudo-kinematic GPS. Erggediggg of the Satefite.

Remondi, B. W. (1985a). Performing centimeter accuracy relative surveys in seconds using GPS carrier phase. In First International Symposium on Precise Positioning with the Global Positioning System, pages 15-19.

Remondi, B. W. (1985b). Performing Centimeter-Level Surveys in Seconds with GPS Carrier Phase: Initial Results. Navigation, 32(4):386-400.

Remondi, B. W. (1991). Kinematic GPS results without static initialization. US Department of Commerce, National Oceanic and Atmospheric Administration, National Ocean Service, Coast and Geodetic Survey.

Reyners, M. (1998). Plate coupling and the hazard of large subduction thrust earthquakes at the Hikurangi subduction zone, New Zealand. New Zealand Journal of Geology and Geophysics, 41(4):343-354.

Reyners, M., Eberhart-Phillips, D., and Martin, S. (2014). Prolonged Canterbury earthquake sequence linked to widespread weakening of strong crust. Nature Geoscience, $7(1): 34-37$.

Reyners, M., Gledhill, K., and Waters, D. (1991). Tearing of the subducted Australian plate during the Te Anau, New Zealand, earthquake of 1988 June 3. Geophysical journal international, 104(1):105-115.

Reyners, M., McGinty, P., Cox, S., Turnbull, I., O Neill, T., Gledhill, K., Hancox, G., Beavan, J., Matheson, D., and McVerry, G. (2003). The $\mathrm{M}^{\sim}$ W 7.2 Fiordland Earthquake of August 21, 2003: Background and Preliminary Results. BULLETINNEW ZEALAND SOCIETY FOR EARTHQUAKE ENGINEERING, 36(4):233248.

Reyners, M. and Webb, T. (2002). Large earthquakes near doubtful sound, New Zealand, 1989-93. New Zealand Journal of Geology and Geophysics, 45(1):109-120. 
Rice, J. R. and Cleary, M. P. (1976). Some basic stress diffusion solutions for fluidsaturated elastic porous media with compressible constituents. Rev. Geophys. Space Phys, 14(2):227-241.

Rice, J. R., Lapusta, N., and Ranjith, K. (2001). Rate and state dependent friction and the stability of sliding between elastically deformable solids. Journal of the Mechanics and Physics of Solids, 49(9):1865-1898.

Riva, R., Aoudia, A., Vermeersen, L., Sabadini, R., and Panza, G. (2000). Crustal versus asthenospheric relaxation and post-seismic deformation for shallow normal faulting earthquakes: the Umbria-Marche (central Italy) case. Geophysical Journal International, 141(3):F7-F11.

Robinson, R., Webb, T., McGinty, P., Cousins, J., and Eberhart-Phillips, D. (2003). The 2000 Thompson Sound earthquake, New Zealand. New Zealand journal of geology and geophysics, 46(3):331-341.

Roeloffs, E. (1996). Poroelastic techniques in the study of earthquake-related hydrologic phenomena. Advances in geophysics, (37):135-195.

Roeloffs, E. A. (1988). Fault stability changes induced beneath a reservoir with cyclic variations in water level. Journal of Geophysical Research: Solid Earth (1978-2012), 93(B3):2107-2124.

Rollins, C., Barbot, S., and Avouac, J.-P. (2015). Postseismic Deformation Following the $2010 \mathrm{M}=7.2$ El Mayor-Cucapah Earthquake: Observations, Kinematic Inversions, and Dynamic Models. Pure and Applied Geophysics, 172(5):1305-1358.

Rosen, P., Hensley, S., Joughin, I. R., Li, F. K., Madsen, S. N., Rodriguez, E., Goldstein, R. M., et al. (2000). Synthetic aperture radar interferometry. Proceedings of the IEEE, 88(3):333-382.

Rousset, B., Barbot, S., Avouac, J.-P., and Hsu, Y.-J. (2012a). Postseismic deformation following the 1999 Chi-Chi earthquake, Taiwan: Implication for lower-crust rheology. Journal of Geophysical Research: Solid Earth (1978-2012), 117(B12).

Rousset, B., Barbot, S., Avouac, J.-P., and Hsu, Y.-J. (2012b). Postseismic deformation following the 1999 Chi-Chi earthquake, Taiwan: Implication for lower-crust rheology. Journal of Geophysical Research B: Solid Earth, 117(12).

Ruina, A. (1983). Slip instability and state variable friction laws. Journal of Geophysical Research: Solid Earth (1978-2012), 88(B12):10359-10370. 
Rundle, J. B. (1980). Static elastic-gravitational deformation of a layered half space by point couple sources. Journal of Geophysical Research: Solid Earth (1978-2012), 85(B10):5355-5363.

Rundle, J. B. (1982). Viscoelastic-gravitational deformation by a rectangular thrust fault in a layered Earth. Journal of Geophysical Research: Solid Earth (1978-2012), 87(B9):7787-7796.

Rybicki, K. (1971). The elastic residual field of a very long strike-slip fault in the presence of a discontinuity. Bulletin of the Seismological Society of America, 61(1):79-92.

Rydelek, P. A. and Pollitz, F. F. (1994). Fossil strain from the 1811-1812 New Madrid earthquakes. Geophysical research letters, 21(21):2303-2306.

Rydelek, P. A. and Sacks, I. S. (2001). Migration of large earthquakes along the San Jacinto fault; stress diffusion from the 1857 Fort Tejon earthquake. Geophysical research letters, 28(16):3079-3082.

Ryder, I., Bürgmann, R., and Sun, J. (2010a). Tandem afterslip on connected fault planes following the 2008 Nima-Gaize (Tibet) earthquake. Journal of Geophysical Research: Solid Earth (1978-2012), 115(B3).

Ryder, I., Brgmann, R., and Sun, J. (2010b). Tandem afterslip on connected fault planes following the 2008 Nima-Gaize (Tibet) earthquake. Journal of Geophysical Research, 115(B3):B03404.

Ryder, I., Wang, H., Bie, L., and Rietbrock, A. (2014). Geodetic imaging of late postseismic lower crustal flow in Tibet. Earth and Planetary Science Letters, 404:136143.

Sato, R. (1971). Crustal deformation due to dislocation in a multi-layered medium. Journal of Physics of the Earth, 19(1):31-46.

Savage, J. (2007). Postseismic relaxation associated with transient creep rheology. Journal of Geophysical Research: Solid Earth (1978-2012), 112(B5).

Savage, J. and Church, J. (1974). Evidence for postearthquake slip in the Fairview Peak, Dixie Valley, and Rainbow Mountain fault areas of Nevada. Bulletin of the Seismological Society of America, 64(3-1):687-698.

Savage, J. and Prescott, W. (1978). Asthenosphere readjustment and the earthquake cycle. Journal of Geophysical Research: Solid Earth (1978-2012), 83(B7):3369-3376. 
Savage, J., Svarc, J., and Prescott, W. (2003). Near-field postseismic deformation associated with the 1992 Landers and 1999 Hector Mine, California, earthquakes. Journal of Geophysical Research: Solid Earth (1978-2012), 108(B9).

Savage, J., Svarc, J., and Yu, S.-B. (2005). Postseismic relaxation and transient creep. Journal of Geophysical Research: Solid Earth (1978-2012), 110(B11).

Sawai, Y., Satake, K., Kamataki, T., Nasu, H., Shishikura, M., Atwater, B. F., Horton, B. P., Kelsey, H. M., Nagumo, T., and Yamaguchi, M. (2004). Transient uplift after a 17th-century earthquake along the Kuril subduction zone. Science, 306(5703):19181920.

Schaff, D. P. and Beroza, G. C. (2004). Coseismic and postseismic velocity changes measured by repeating earthquakes. Journal of Geophysical Research: Solid Earth, 109(B10).

Schapery, R. (1967). Stress analysis of viscoelastic composite materials. Journal of Composite Materials, 1(3):228-267.

Schmid, R., Steigenberger, P., Gendt, G., Ge, M., and Rothacher, M. (2007). Generation of a consistent absolute phase-center correction model for GPS receiver and satellite antennas. Journal of Geodesy, 81(12):781-798.

Scholz, C. H. (1974). Post-earthquake dilatancy recovery. Geology, 2(11):551-554.

Segall, P. (2010). Earthquake and volcano deformation. Princeton University Press.

Segall, P. and Matthews, M. (1997). Time dependent inversion of geodetic data. Journal of Geophysical Research: Solid Earth (1978-2012), 102(B10):22391-22409.

Sheu, S.-Y. and Shieh, C.-F. (2004). Viscoelastic-afterslip concurrence: a possible mechanism in the early post-seismic deformation of the Mw 7.6, 1999 Chi-Chi (Taiwan) earthquake. Geophysical Journal International, 159(3):1112-1124.

Sibson, R., Ghisetti, F., and Ristau, J. (2011). Stress control of an evolving strike-slip fault system during the 2010-2011 Canterbury, New Zealand, earthquake sequence. Seismological Research Letters, 82(6):824-832.

Sibson, R. H. (1986). Rupture interaction with fault jogs. Earthquake Source Mechanics, $37: 157-167$.

Singh, S. J. (1970). Static deformation of a multilayered half-space by internal sources. Journal of Geophysical Research, 75(17):3257-3263. 
Smith, E. G. and Davey, F. J. (1984). Joint hypocentre determination of intermediate depth earthquakes in Fiordland, New Zealand. Tectonophysics, 104(1):127-144.

Strang, G. and Fix, G. J. (1973). An analysis of the finite element method, volume 212. Prentice-Hall Englewood Cliffs, NJ.

Suito, H. and Freymueller, J. T. (2009). A viscoelastic and afterslip postseismic deformation model for the 1964 Alaska earthquake. Journal of Geophysical Research: Solid Earth (1978-2012), 114(B11).

Suito, H. and Hirahara, K. (1999). Simulation of postseismic deformations caused by the 1896 Riku-u Earthquake, northeast Japan: Re-evaluation of the viscosity in the upper mantle. Geophysical research letters, 26(16):2561-2564.

Sun, T. and Wang, K. (2015). Viscoelastic relaxation following subduction earthquakes and its effects on afterslip determination. Journal of Geophysical Research: Solid Earth.

Sutherland, R., Davey, F., and Beavan, J. (2000). Plate boundary deformation in South Island, New Zealand, is related to inherited lithospheric structure. Earth and Planetary Science Letters, 177(3):141-151.

Sutherland, R. and Norris, R. (1995). Late Quaternary displacement rate, paleoseismicity, and geomorphic evolution of the Alpine Fault: evidence from Hokuri Creek, South Westland, New Zealand. New Zealand journal of geology and geophysics, 38(4):419-430.

Sylvester, J. (1884). Sur léquation en matrices px=xq. CR Acad. Sci. Paris, 99(6771):115-116.

Syracuse, E., Holt, R., Savage, M., Johnson, J., Thurber, C., Unglert, K., Allan, K., Karaliyadda, S., and Henderson, M. (2012). Temporal and spatial evolution of hypocentres and anisotropy from the Darfield aftershock sequence: implications for fault geometry and age. New Zealand Journal of Geology and Geophysics, 55(3):287293.

Tabrez, A. S., Freed, A. M., Calais, E., Manaker, D. M., and McCann, W. R. (2008). Coulomb stress evolution in Northeastern Caribbean over the past 250 years due to coseismic, postseismic and interseismic deformation. Geophysical Journal International, 174(3):904-918.

Tanaka, Y., Okuno, J., and Okubo, S. (2006). A new method for the computation of global viscoelastic post-seismic deformation in a realistic earth model (I)vertical 
displacement and gravity variation. Geophysical Journal International, 164(2):273289.

Terzaghi, K. (1943). Theoretical soil mechanics. Wiley.

Townend, J., Sherburn, S., Arnold, R., Boese, C., and Woods, L. (2012). Threedimensional variations in present-day tectonic stress along the Australia-Pacific plate boundary in New Zealand. Earth and Planetary Science Letters, 353:47-59.

Tullis, T., e. a. (2007). Group report: Rheology of fault rocks and their surroundings, in Tectonic Faults Agents of Change on a Dynamic Earth, edited by M.R. Handy, G. Hirth, N. Hovius, Dahlem Workshop Report 95, 504 pp. The MIT Press, Cambridge, Mass., USA.

Turcotte, D. L. and Schubert, G. (2014). Geodynamics. Cambridge University Press.

Ueda, H., Ohtake, M., and Sato, H. (2003). Postseismic crustal deformation following the 1993 Hokkaido Nansei-oki earthquake, northern Japan: Evidence for a lowviscosity zone in the uppermost mantle. Journal of Geophysical Research: Solid Earth (1978-2012), 108(B3).

Van Ballegooy, S., Cox, S. C., Agnihotri, R., Reynolds, T., Thurlow, C., Rutter, H., Scott, D., Begg, J., and McCahon, I. (2013). Median water table elevation in Christchurch and surrounding area after the 4 September 2010 Darfield Earthquake.

Van Ballegooy, S., Malan, P., Lacrosse, V., Jacka, M., Cubrinovski, M., Bray, J., O'Rourke, T., Crawford, S., and Cowan, H. (2014). Assessment of liquefactioninduced land damage for residential Christchurch. Earthquake Spectra, 30(1):31-55.

Van Dissen, R. and Yeats, R. S. (1991). Hope fault, Jordan thrust, and uplift of the seaward Kaikoura Range, New Zealand. Geology, 19(4):393-396.

van Hunen, J., Zhong, S., Shapiro, N. M., and Ritzwoller, M. H. (2005). New evidence for dislocation creep from 3-D geodynamic modeling of the Pacific upper mantle structure. Earth and Planetary Science Letters, 238(1):146-155.

Van Sickle, J. (2008). GPS for land surveyors. Crc Press.

Vergnolle, M., Pollitz, F., and Calais, E. (2003). Constraints on the viscosity of the continental crust and mantle from GPS measurements and postseismic deformation models in western Mongolia. Journal of Geophysical Research: Solid Earth (19782012), 108(B10). 
Vidale, J. E. and Li, Y.-G. (2003). Damage to the shallow Landers fault from the nearby Hector Mine earthquake. Nature, 421(6922):524-526.

Vladimirova, I., Steblov, G., and Frolov, D. (2011). Viscoelastic deformations after the 2006-2007 Simushir earthquakes. Izvestiya, Physics of the Solid Earth, 47(11):10201025 .

Wahr, J. and Wyss, M. (1980). Interpretation of postseismic deformation with a viscoelastic relaxation model. Journal of Geophysical Research: Solid Earth (19782012), 85(B11):6471-6477.

Walcott, R. (1998). Modes of oblique compression: Late Cenozoic tectonics of the South Island of New Zealand. Reviews of Geophysics, 36(1):1-26.

Wald, D. J. and Graves, R. W. (2001). Resolution analysis of finite fault source inversion using one-and three-dimensional Green's functions: 2. Combining seismic and geodetic data. Journal of Geophysical Research: Solid Earth (1978-2012), 106(B5):8767-8788.

Wallace, L. M. and Beavan, J. (2010). Diverse slow slip behavior at the Hikurangi subduction margin, New Zealand. Journal of Geophysical Research: Solid Earth (1978-2012), 115(B12).

Wallace, L. M., Beavan, J., McCaffrey, R., and Darby, D. (2004). Subduction zone coupling and tectonic block rotations in the North Island, New Zealand. Journal of Geophysical Research: Solid Earth (1978-2012), 109(B12).

Walls, J. and Nur, A. (1979). Pore pressure and confining pressure dependence of permeability in sandstone. In Transactions of the 7th Formation Evaluation Symposium, pages $1-8$.

Wandres, A. M., Bradshaw, J. D., and Ireland, T. (2005). The Paleozoic-Mesozoic recycling of the Rakaia Terrane, South Island, New Zealand: Sandstone clast and sandstone petrology, geochemistry, and geochronology. New Zealand journal of geology and geophysics, 48(2):229-245.

Wandres, A. M., Bradshaw, J. D., Weaver, S., Maas, R., Ireland, T., and Eby, N. (2004). Provenance analysis using conglomerate clast lithologies: a case study from the Pahau terrane of New Zealand. Sedimentary Geology, 167(1):57-89.

Wang, H. (2000). Theory of linear poroelasticity with applications to geomechanics and hydrogeology. Princeton University Press. 
Wang, K., Hu, Y., and He, J. (2012). Deformation cycles of subduction earthquakes in a viscoelastic Earth. Nature, 484(7394):327-332.

Wang, L., Wang, R., Roth, F., Enescu, B., Hainzl, S., and Ergintav, S. (2009). Afterslip and viscoelastic relaxation following the 1999 M 7.4 Izmit earthquake from GPS measurements. Geophysical Journal International, 178(3):1220-1237.

Wang, R. (2005). The dislocation theory: a consistent way for including the gravity effect in (visco) elastic plane-earth models. Geophysical Journal International, 161(1):191-196.

Wang, R. and Kümpel, H.-J. (2003). Poroelasticity: Efficient modeling of strongly coupled, slow deformation processes in a multilayered half-space. Geophysics, 68(2):705717.

Wang, R., Lorenzo-Martín, F., and Roth, F. (2006). PSGRN/PSCMPa new code for calculating co-and post-seismic deformation, geoid and gravity changes based on the viscoelastic-gravitational dislocation theory. Computers \& Geosciences, 32(4):527541.

Watson, G. N. (1995). A treatise on the theory of Bessel functions. Cambridge university press.

Wdowinski, S., Bock, Y., Zhang, J., Fang, P., Genrich, J., et al. (1997). Southern California permanent GPS geodetic array: spatial filtering of daily positions for estimating coseismic and postseismic displacements induced by the 1992 Landers earthquake. Journal of Geophysical Research-Part B-Solid Earth-Printed Edition, 102(8):18057-18070.

Wen, Y., Li, Z., Xu, C., Ryder, I., and Brgmann, R. (2012a). Postseismic motion after the 2001 Mw7.8 kokoxili earthquake in Tibet observed by InSAR time series. Journal of Geophysical Research B: Solid Earth, 117(8).

Wen, Y., Li, Z., Xu, C., Ryder, I., and Bürgmann, R. (2012b). Postseismic motion after the 2001 MW 7.8 Kokoxili earthquake in Tibet observed by InSAR time series. Journal of Geophysical Research: Solid Earth (1978-2012), 117(B8).

Whitaker, S. (1986). Flow in porous media I: A theoretical derivation of Darcy's law. Transport in porous media, 1(1):3-25.

White, S. M., Trenkamp, R., and Kellogg, J. N. (2003). Recent crustal deformation and the earthquake cycle along the Ecuador-Colombia subduction zone. Earth and Planetary Science Letters, 216(3):231-242. 
Wu, P. (1992). Deformation of an incompressible viscoelastic flat earth with powerlaw creep: a finite element approach. Geophysical journal international, 108(1):35-51.

Wu, P. (2004). Using commercial finite element packages for the study of earth deformations, sea levels and the state of stress. Geophysical Journal International, 158(2):401-408.

Yabuki, T. and Matsu'Ura, M. (1992). Geodetic data inversion using a Bayesian information criterion for spatial distribution of fault slip. Geophysical Journal International, 109(2):363-375.

Yamagiwa, S., Miyazaki, S., Hirahara, K., and Fukahata, Y. (2015). Afterslip and viscoelastic relaxation following the 2011 Tohoku-oki earthquake (Mw9. 0) inferred from inland GPS and seafloor GPS/Acoustic data. Geophysical Research Letters.

Yamasaki, T., Wright, T. J., and Houseman, G. A. (2014). Weak ductile shear zone beneath a major strike-slip fault: Inferences from earthquake cycle model constrained by geodetic observations of the western North Anatolian Fault Zone. Journal of Geophysical Research: Solid Earth, 119(4):3678-3699.

Zebker, H. A., Rosen, P. A., and Hensley, S. (1997). Atmospheric effects in interferometric synthetic aperture radar surface deformation and topographic maps. Journal of Geophysical Research: Solid Earth, 102(B4):7547-7563.

Zebker, H. A. and Villasenor, J. (1992). Decorrelation in interferometric radar echoes. Geoscience and Remote Sensing, IEEE Transactions on, 30(5):950-959.

Zhong, S., Paulson, A., and Wahr, J. (2003). Three-dimensional finite-element modelling of Earths viscoelastic deformation: effects of lateral variations in lithospheric thickness. Geophysical Journal International, 155(2):679-695.

Zoback, M. D. and Byerlee, J. (1975). Permeability and effective stress: Geologic notes. AAPG Bulletin, 59(1):154-158. 